\title{
Design and optimization of large stroke flexure mechanisms
}

\section{Mark Naves}

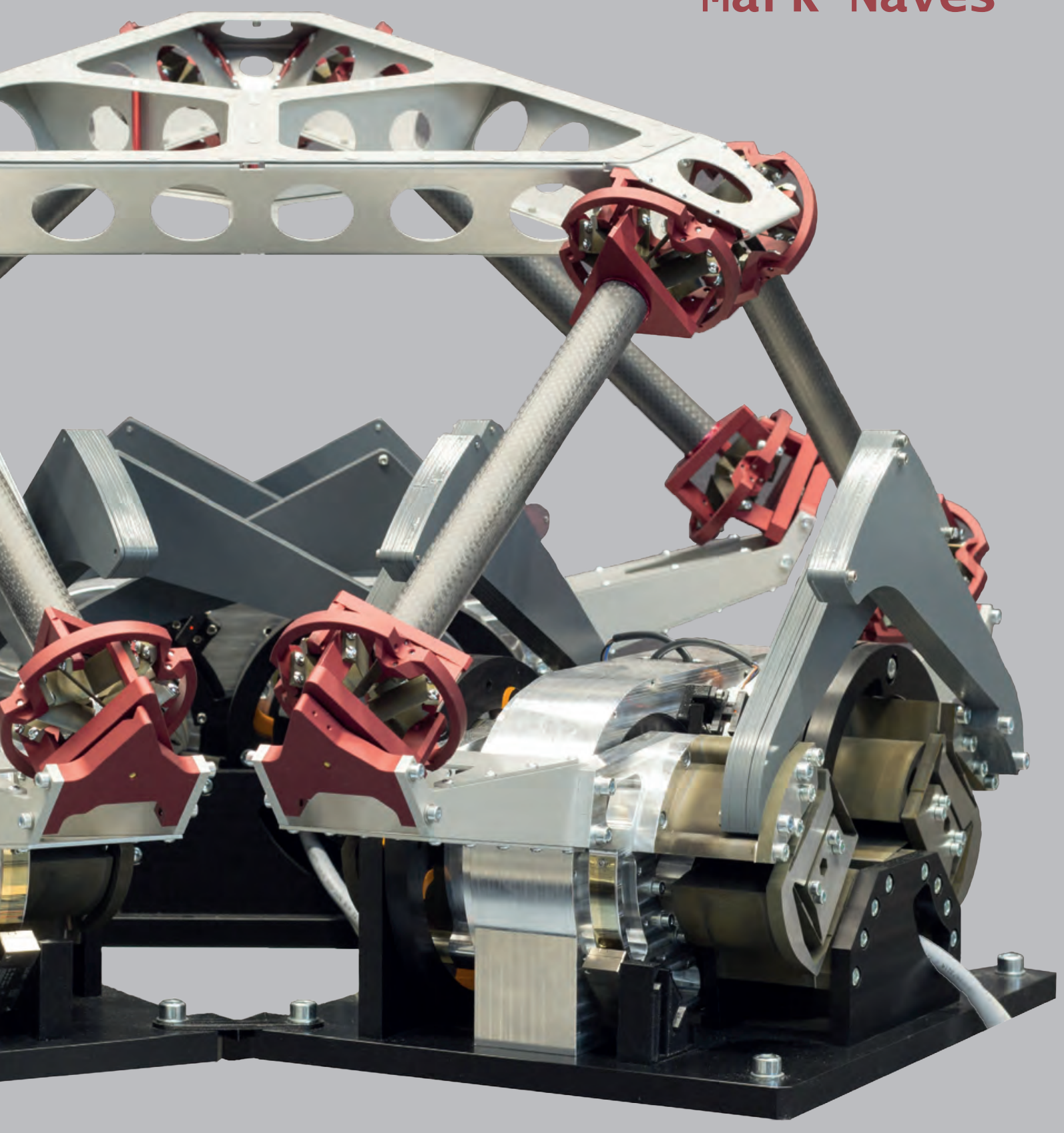





\title{
Design and optimization of large stroke flexure mechanisms
}

\author{
Mark Naves
}


The work described in this thesis was performed at the Chair of Precision Engineering of the Faculty of Engineering Technology, University of Twente, P.O. Box 217, 7500 AE Enschede, the Netherlands.

This research was financially supported by the Innovative Research Incentives Scheme VIDI (Stichting voor de Technische Wetenschappen) (14152 NWO TTW) of the ministry of Education, Culture and Science of the Netherlands. The author would also like to thank the following companies for their support and technical advice in this project: Heidenhain Netherlands B.V., Ede; SigmaControl B.V., Barendrecht; Tecnotion B.V., Almelo; VDL Enabling Technologies Group B.V., Almelo.

Cover design by Jalisa Leuverink

Design and optimization of large stroke flexure mechanisms M. Naves

$\mathrm{PhD}$ Thesis, University of Twente, Enschede, the Netherlands

ISBN 978-90-365-4994-3

DOI 10.3990/1.9789036549943

(C)2021 Mark Naves, the Netherlands. All rights reserved. No parts of this thesis may be reproduced, stored in a retrieval system or transmitted in any form or by any means without permission of the author. Alle rechten voorbehouden. Niets uit deze uitgave mag worden vermenigvuldigd, in enige vorm of op enige wijze, zonder voorafgaande schriftelijke toestemming van de auteur. 


\title{
DESIGN AND OPTIMIZATION OF LARGE STROKE FLEXURE MECHANISMS
}

\section{DISSERTATION}

\author{
to obtain \\ the degree of doctor at the University of Twente, \\ on the authority of the rector magnificus, \\ prof. dr. ir. A. Veldkamp, \\ on account of the decision of the Doctorate Board \\ to be publicly defended \\ on Friday 21 May 2021 at 14:45
}

by

\section{Mark Naves}

born on the 27th of April 1992 in Winterswijk, the Netherlands 
This dissertation has been approved by:

Supervisor:

prof. dr. ir. D.M. Brouwer PDEng

Co-supervisors:

dr. ir. R.G.K.M. Aarts

dr. ir. W.B.J. Hakvoort 


\section{Graduation Committee:}

Chairman / secretary: prof. dr. ir. H.F.J.M. Koopman University of Twente Supervisor: $\quad$ prof. dr. ir. D.M. Brouwer PDEng University of Twente

Co-supervisors: $\quad$ dr. ir. R.G.K.M. Aarts

University of Twente dr. ir. W.B.J. Hakvoort University of Twente

Committee Members: $\quad$ prof. dr. ir. J.P.M.B. Vermeulen

Eindhoven University of Technology

prof. dr. ir. J.L. Herder

Delft University of Technology

prof. dr. ir. A.H. van den Boogaard

dr. ir. R. Loendersloot

University of Twente

University of Twente 
Never memorize something that you can look up.

Albert Einstein 


\section{Samenvatting}

Elastische mechanismen worden veelvuldig gebruikt voor precisietoepassingen vanwege hun deterministische eigenschappen door het ontbreken van speling en wrijving. Voor toepassingen met een relatief groot werkbereik worden elastische mechanismen vaak vermeden vanwege de grote inzakking van de stijfheid en belastbaarheid in de ondersteuningsrichtingen bij grote vervormingen. De prestaties van elastische mechanismen voor toepassingen met grote slag kunnen worden verbeterd door gebruik te maken van een complexere geometrie die in staat is om stijfheid te behouden over het gehele werkbereik. Echter, het verkrijgen van een goed ontwerp voor een elastisch mechanisme geschikt voor grote slag toepassingen is niet triviaal ten gevolge van de complexe relatie tussen de geometrie van de elastische elementen en de prestaties van het systeem, zeker bij de optredende grote vervormingen.

Een oplossing voor dit probleem wordt gepresenteerd in dit proefschrift in de vorm van een optimalisatiestrategie voor elastische mechanismen. Deze strategie maakt gebruik van een optimalisatie-algoritme gebaseerd op de NelderMead methode gecombineerd met ontwerprichtlijnen voor elastische mechanismen. Dit met het doel om nieuwe en verbeterde ontwerpen te verkrijgen voor grote slag elastische mechanismen. De optimalisatiestrategie is aangevuld met een botsingsdetectie-algoritme om de optimalisatie van complexe ruimtelijke elastische mechanismen mogelijk te maken waarbij de individuele elastische elementen niet met elkaar in contact komen. Deze aanpak heeft geleid tot een geoptimaliseerd ontwerp voor een grote slag elastisch scharnier met éen vrije rotatierichting en een werkbereik van \pm 45 graden rotatie, een universeel gewricht met twee vrije rotatierichtingen en een werkbereik van \pm 25 graden kantelhoek en een kogelgewricht met een werkbereik van \pm 30 kantelhoek en \pm 10 graden draaiingshoek. De verkregen ontwerpen doorbreken de traditioneel veronderstelde maximale uitwijkingen van slechts enkele graden en bieden een ongeëvenaarde stijfheid bij uitwijking. Hierdoor bieden deze gewrichten een elastisch alternatief ter vervanging van traditionele lageringen in systemen die verhoogde precisie vereisen in combinatie met een groot werkbereik. 
Om de potentie van de ontwikkelde grote slag elastische gewrichten te demonstreren is een volledig elastische hexapod robot met 6 graden van bewegingsvrijheid ontworpen en geoptimaliseerd. Door gebruik te maken van de verkregen ontwerpen voor grote slag elastische gewrichten is een groot werkbereik van \pm 100 millimeter in de translatierichtingen en meer dan \pm 10 graden in de rotatierichtingen gerealiseerd waarbij de afmetingen van het systeem beperkt blijven tot een grondradius van 0.43 meter en een hoogte van 0.42 meter. Daarnaast zijn hoge actuatiekrachten mogelijk zonder verlies van nauwkeurigheid door een speciaal ontwerp voor de elastische ophanging voor de actuatoren met geringe verandering van de locatie van de rotatie-as, wat het systeem in staat stelt tot versnellingen boven de $10 \mathrm{~g}$ en krachtterugkoppeling op de motoren.

Topologie en ontwerpoptimalisaties laten zien dat er nieuwe mogelijkheden zijn voor grote slag elastische mechanismen doordat een hogere ondersteuningstijfheid behaald kan worden bij grote uitwijkingen. Dit biedt mogelijkheden voor het vergroten van het werkbereik of het verbeteren van de verhouding tussen het werkbereik en de inbouwruimte van het mechanisme zonder last te hebben van een sterke vermindering van de ondersteuningsstijfheid. Daarnaast geeft het vergrote werkbereik een verbreding van het toepassingsgebied, wat nieuwe potentie geeft voor het gebruik van elastische mechanismen. 


\section{Summary}

Flexure-based mechanisms are widely used in many small stroke precision applications because of the absence of play and friction, resulting in highly deterministic and predictable behavior. However, for large stroke applications, flexure-based mechanisms are often avoided due to the strong decrease in support stiffness and load-bearing capacity when subjected to large deflections. The performance of flexure-based mechanisms for large range of motion applications can be improved by using more complex geometries to maintain high support stiffness at larger deflections. However, due to the complex relation between the geometry of the flexures and the performance of the system, particularly in the presence of large deformations, designing a "good" flexure mechanism suited for large stroke applications is not trivial.

For this reason, a strategy for the optimization of large stroke flexure-based mechanisms is presented in this thesis. This strategy takes advantage of a NelderMead based shape optimization algorithm combined with design principles for flexure-based systems in order to synthesize new design topologies for flexurebased equivalents of traditional bearings. This optimization strategy is supplemented by a collision detection scheme to enable the optimization of complex spatial mechanisms while avoiding collision of the flexures. This approach has been used to devise and optimize a new design for a large stroke flexure-based revolute joint with a range of motion of \pm 45 degrees rotation, a universal joint with a range of motion of \pm 25 degrees tip-tilt motion and a spherical joint with a range of motion of \pm 30 degrees tip-tilt and \pm 10 degrees pan motion. The obtained designs break through the traditionally considered maximum deflection angles of only a few degrees and provide unmatched support stiffness at large deflections. As a result, these flexure joints can be used as a "stand-in" replacement of traditional bearings for systems which require enhanced precision and large rotation angles.

In order to demonstrate the potential of these large range of motion joints, a fully flexure-based hexapod robot with 6 degrees of freedom has been designed and optimized. By utilizing the obtained designs for the large range of motion universal and spherical joints, an unprecedented range of motion for a flexure- 
based hexapod robot is obtained. This hexapod allows for a translational range of motion of \pm 100 millimeter and more than \pm 10 degrees of rotation in each direction combined with a base radius of 0.43 meter and a height of 0.42 meter. Furthermore, a dedicated low pivot shift flexure-based design for the actuators enable the use of high actuation forces without impairing precision, allowing for end effector accelerations exceeding $10 \mathrm{~g}$ and force feedback at the actuators.

Topology and shape optimization show new possibilities for large stroke flexure mechanisms by enabling higher support stiffness at large deflection. This allows for applications with a larger range of motion or improved mechanism volume to workspace ratio without suffering from a strong reduction in support stiffness when deflected. Furthermore, the increased range of motion allows for a broadening of the scope of application, expanding the potential for the use of flexures-based mechanisms. 


\section{Notation and math operators}

$a, b, \ldots \quad$ Real numbers

$A, B, \ldots \quad$ Real numbers

$\boldsymbol{a}, \boldsymbol{b}, \ldots \quad$ Vectors

$\boldsymbol{A}, \boldsymbol{B}, \ldots$ Vectors or matrices

$A(s), \ldots \quad$ Transfer function in the Laplace domain

$\mathcal{F} \quad$ Optimization objective function

$\mathcal{C} \quad$ Optimization constraint function

$\|\boldsymbol{A}\| \quad$ Euclidean norm (2-norm) of vector $\boldsymbol{A}$

$\boldsymbol{A} \cdot \boldsymbol{B} \quad$ Dot product of vector $\boldsymbol{A}$ and $\boldsymbol{B}$

$\boldsymbol{A} \times \boldsymbol{B} \quad$ Cross product of vector $\boldsymbol{A}$ and $\boldsymbol{B}$

$\mathbb{A}, \mathbb{B}, \ldots \quad$ Surfaces

$\overleftarrow{A B} \quad$ Infinite line through point $A$ and $B$

$\overline{A B} \quad$ Line segment between point $A$ and $B$ 



\section{Contents}

Samenvatting v v

Summary vii

\begin{tabular}{|l|l}
\hline Notation and math operators & ix
\end{tabular}

Contents

$\begin{array}{lll}1 & \text { Introduction } & 1\end{array}$

1.1 Background . . . . . . . . . . . . . . . . 1

1.2 Research objectives $\ldots \ldots \ldots \ldots \ldots \ldots$

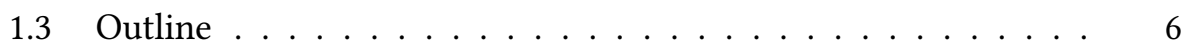

\begin{tabular}{|l|l|}
\hline Design and optimization of large stroke flexure joints & 11
\end{tabular}

2 Building block-based spatial topology synthesis method for largestroke flexure hinges 11

2.1 Introduction . . . . . . . . . . . . . . . . 11

$2.2 \quad$ Shape optimization method . . . . . . . . . . . . . . . 14

2.2 .1 Nelder-Mead method . . . . . . . . . . . . . . . . . . 14

2.2 .2 Adaptive objective function . . . . . . . . . . . . 15

2.2 .3 Constraint interpolation . . . . . . . . . . . . . . . . 17

2.2 .4 Shape optimization performance $\ldots \ldots \ldots \ldots$

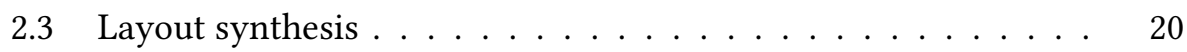

2.3 .1 Building blocks . . . . . . . . . . . . . . 20

2.3 .2 Building block overview . . . . . . . . . . . . . . . 23

2.3 .3 Layout update strategy $\ldots \ldots \ldots \ldots \ldots$

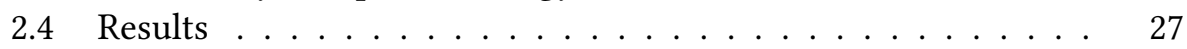

$2.4 .1 \quad$ Optimization case . . . . . . . . . . . . . . . . . . . 27

$2.4 .2 \quad$ Experimental validation $\ldots \ldots \ldots \ldots$

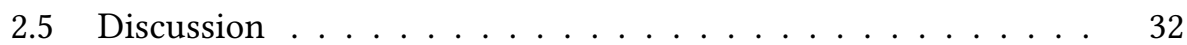


2.6 Conclusion . . . . . . . . . . . . . . . . . . . 32

2.7 Appendices . . . . . . . . . . . . . . . . . . . . 34

2.7.1 Optimization of a large stroke flexure hinge for off-axis support stiffness $\ldots \ldots \ldots \ldots \ldots \ldots \ldots \ldots \ldots$

2.7 .2 Intermediate optimization results $\ldots \ldots \ldots \ldots$

3 Efficient collision detection method for flexure mechanisms comprising deflected leafsprings 39

3.1 Introduction . . . . . . . . . . . . . . . . . . 39

3.2 Collision test . . . . . . . . . . . . . . . . . 41

3.2 .1 Geometrical shape reduction . . . . . . . . . . . 42

3.2 .2 Initial bounding volume test $\ldots \ldots \ldots \ldots \ldots \ldots$. . . . . . 42

3.2 .3 Geometry intersection test. . . . . . . . . . . . . . 46

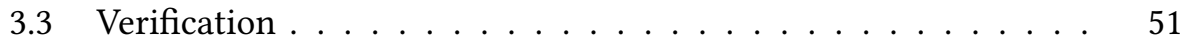

$3.3 .1 \quad$ Collision test continuity $\ldots \ldots \ldots \ldots \ldots$. . . . . 51

3.3 .2 Computational efficiency. . . . . . . . . . . . 52

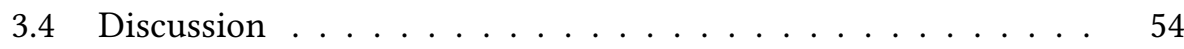

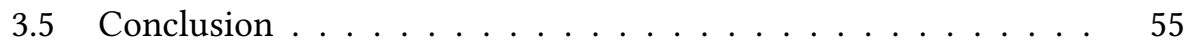

$4 \quad$ Large stroke high off-axis stiffness three degree of freedom spherical flexure joint $\quad 57$

4.1 Introduction . . . . . . . . . . . . . . . . 57

4.2 Notational convention for spherical motion. . . . . . . . . . 59

4.3 Controlling degrees of freedom $\ldots \ldots \ldots \ldots$

$4.4 \quad$ Spherical joint concept topologies . . . . . . . . . . . . . . 61

$4.4 .1 \quad$ Folded Leafspring based spherical joint (FL joint) . . . . 61

4.4.2 $\quad$ Parallel stacked Folded Leafspring based spherical joint

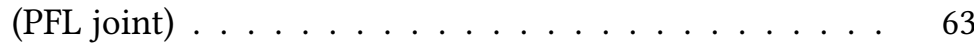

4.4.3 Serial stacked Folded Leafspring based spherical joint (SFL

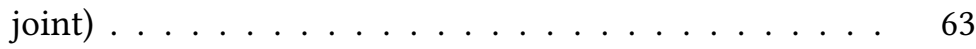

4.5 Shape optimization $\ldots \ldots \ldots \ldots \ldots \ldots \ldots$

$4.5 .1 \quad$ Optimization method . . . . . . . . . . . . . . . 65

$4.5 .2 \quad$ Optimization results $\ldots \ldots \ldots \ldots$

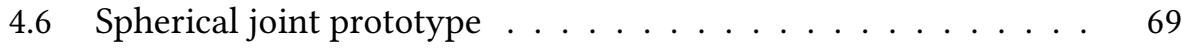

4.6 .1 Prototype design . . . . . . . . . . . . . . . . 69

4.6 .2 Prototype performance. . . . . . . . . . . . . . 70

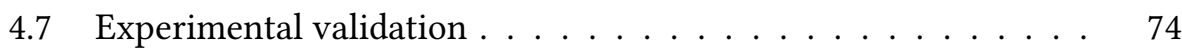

$4.7 .1 \quad$ Experimental setup . . . . . . . . . . . . . . . . 74

$4.7 .2 \quad$ Stiffness validation $\ldots \ldots \ldots \ldots \ldots \ldots$

4.7 .3 Intermediate body dynamics . . . . . . . . . . . 77 
4.8 Suppressing intermediate body vibrations. . . . . . . . . . . 79

$4.8 .1 \quad$ Adding damping . . . . . . . . . . . . . . . . . 79

4.8 .2 Adding a slaving mechanism . . . . . . . . . . . . 80

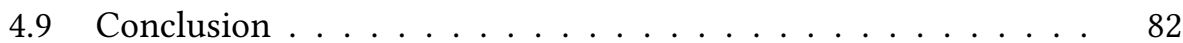

5 Large stroke high off-axis stiffness two degree of freedom uni$\begin{array}{ll}\text { versal flexure joint } & 83\end{array}$

5.1 Introduction . . . . . . . . . . . . . . . . . . . . 83

$5.2 \quad$ Conceptual design $\ldots \ldots \ldots \ldots \ldots \ldots \ldots$

5.3 Performance comparison . . . . . . . . . . . . . . . . . . 87

5.4 Universal joint prototype . . . . . . . . . . . . . . . . . . . . 89

5.5 Discussion $\ldots \ldots \ldots \ldots \ldots \ldots$

5.6 Conclusion $\ldots \ldots \ldots \ldots \ldots$

$5.7 \quad$ Appendix . . . . . . . . . . . . . . . . . . . . . 92

5.7.1 The $\mathrm{O}^{3} \mathrm{XY}^{3}$ universal flexure joint in torque transmitting

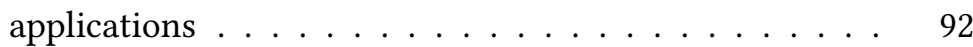

Case study: The T-Flex, a large range of motion flexure-based hexapod

6 Flexure-based 60 degrees stroke actuator suspension for a high $\begin{array}{ll}\text { torque iron core motor } & 97\end{array}$

6.1 Introduction . . . . . . . . . . . . . . . . . . . . 98

6.2 System description . . . . . . . . . . . . . . . . . . . . . . . 99

6.2 .1 Requirements . . . . . . . . . . . . . . . . . . . . . . 99

6.2 .2 Actuator selection . . . . . . . . . . . . . . . . . 100

6.3 Parasitic motion of revolute flexure joints . . . . . . . . . . . 101

6.3.1 Parasitic motion of the cross-spring pivot . . . . . . . 102

6.3 .2 The cartwheel hinge . . . . . . . . . . . . . . . . . . . 104

6.3 .3 The butterfly hinge . . . . . . . . . . . . . . . . . . 105

$6.4 \quad$ Numerical optimization $\ldots \ldots \ldots \ldots \ldots$. . . . . . . . . . . . 106

$6.4 .1 \quad$ Optimization procedure . . . . . . . . . . . . . . . . 106

$6.4 .2 \quad$ Optimization results . . . . . . . . . . . . . . . . . . 107

6.5 Influence of negative rotor stiffness $\ldots \ldots \ldots \ldots$

6.5.1 Simulation results including negative rotor stiffness . . 109

6.5 .2 Load capacity . . . . . . . . . . . . . . . . . . . . . 111

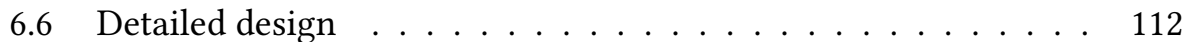

6.6 .1 Butterfly hinge design . . . . . . . . . . . . . . . 113

6.6 .2 Actuator suspension design . . . . . . . . . . . . . . . . 114 
6.6 .3 Controller design . . . . . . . . . . . . . . . . 116

6.7 Experimental validation . . . . . . . . . . . . . . . . 117

6.7 .1 Parasitic motion . . . . . . . . . . . . . 117

$6.7 .2 \quad$ Support stiffness . . . . . . . . . . . . . . . . . . . . . . . . . . . . 118

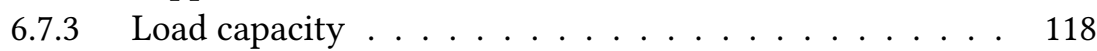

6.7 .4 Repeatability . . . . . . . . . . . . . . . . . . . . . . . . . . . 120

6.8 Discussion . . . . . . . . . . . . . . . . . 121

6.9 Conclusion . . . . . . . . . . . . . . . . . . 122

\begin{tabular}{|ll|l|}
\hline 7 & T-Flex: A fully flexure-based large range of motion precision & \\
\hline \hline hexapod & 123 \\
\hline
\end{tabular}

7.1 Introduction . . . . . . . . . . . . . . . . . . . . 123

7.2 Hexapod layout $\ldots \ldots \ldots$. . . . . . . . . . . . . . . . . . . . . . . . . . . . . . . . . 126

$7.3 \quad$ Optimization . . . . . . . . . . . . . . . . . . 127

7.3.1 Hexapod optimization strategy . . . . . . . . . . . . . 127

7.3.2 $\quad$ Actuated revolute base joint optimization . . . . . . . . 129

7.3.3 $\quad$ Spherical/universal joint optimization . . . . . . . . . . 132

7.3.4 Hexapod design optimization . . . . . . . . . . . . . 135

7.4 Optimization results . . . . . . . . . . . . . . . . . . . . . . . . . . . . . 137

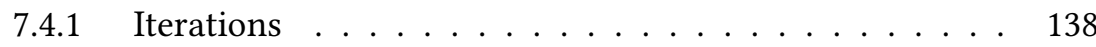

7.4 .2 Discussion . . . . . . . . . . . . . . . 139

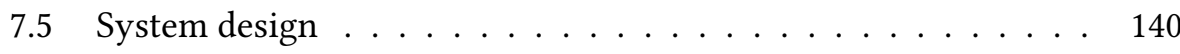

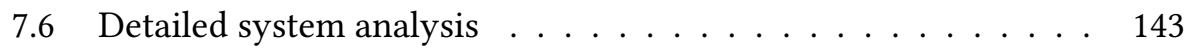

7.6.1 Workspace and eigenfrequencies . . . . . . . . . . . 143

7.6 .2 Maximum accelerations . . . . . . . . . . . . . . 144

7.6 .3 Maximum load capacity . . . . . . . . . . . . . . 145

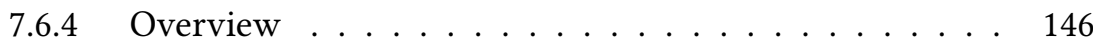

$7.7 \quad$ Prototype design . . . . . . . . . . . . . . . . . . . . 148

7.7.1 Mechanical design . . . . . . . . . . . . . . . . 148

7.7.2 $\quad$ System identification . . . . . . . . . . . . . . . . . 149

7.7.3 Controller design . . . . . . . . . . . . . . . . . . . 151

7.8 Experimental validation $\ldots \ldots \ldots$. . . . . . . . . . . . . . . . . . . . . . . . . . 153

7.8 .1 The standstill performance . . . . . . . . . . . . . . 153

7.8.2 Repeatability . . . . . . . . . . . . . 154

7.9 Discussion . . . . . . . . . . . . . . . . . . 156

7.10 Conclusion . . . . . . . . . . . . . . . . . . 157

7.11 Appendices . . . . . . . . . . . . . . . . . 159

7.11 .1 Mass and inertia properties . . . . . . . . . . . . . 159

7.11 .2 First parasitic eigenfrequency . . . . . . . . . . . . . 160

7.11 .3 Maximum accelerations . . . . . . . . . . . . . 161 
8 Conclusions and recommendations $\quad \mathbf{1 6 5}$

8.1 Conclusions . . . . . . . . . . . . . . . . 165

8.2 Discussion and Recommendations . . . . . . . . . . . . 167

\begin{tabular}{lr}
\hline Bibliography & 169
\end{tabular}

\begin{tabular}{lr}
\hline Research contributions & 179
\end{tabular}

\begin{tabular}{lc}
\hline Dankwoord & 183
\end{tabular} 



\section{Chapter}

\section{Introduction}

This chapter constitutes an introduction to the two main subjects of this thesis: the design and the optimization of flexure-based mechanisms. The design of flexurebased mechanisms always have been of great interest for the development of precision systems as the underlying physics allow for highly predictable and repeatable behavior. However, to improve performance and to extend application potential of these precision systems, the requirements on the range of motion, load capacity and support stiffness of the flexure-based components become increasingly challenging. Because existing traditional designs for flexure-based mechanisms are not always able to meet these demands, this thesis investigates new design solutions for flexurebased systems aimed at improving support stiffness and increasing range of motion. Improved performance can be achieved at the cost of increased complexity, which makes obtaining a "good" design not always trivial. Therefore, optimization strategies are investigated and put to use in order to acquire improved mechanism designs and to allow for a valid comparison between them.

\subsection{Background}

\section{Flexure-based mechanisms}

In mechanics bearings are used to constrain motion in certain directions while allowing for "free" motion in other unconstrained directions. Typically used sliding- or rolling-element bearings provide a cost-efficient solution. However, as those bearings rely on tribological contacts, they do suffer from wear, friction and backlash impairing precision. In contrast, flexure-based mechanisms allow motion by means of elastic material deformation [12, 39, 40, 90, 92]. By taking advantage of the specific shape of flexible elements, e.g. long and slender, motion 
is constrained in certain directions while allowing for almost free motion in other unconstrained directions [29, 31, 103]. As the motion of flexure-based mechanisms relies solely on the elastic deformation of material, they do not suffer from friction and backlash resulting in an excellent repeatability which makes them popular for use in high-precision applications.

Fundamentally, designers of flexure-based mechanisms face a trade-off between flexibility for motion in certain desired directions, and stiffness to constrain motion for guiding in the remaining directions. This trade-off for flexure-based mechanisms subjected to only small deflections can be made by means of simple linear beam-deflection formulas. However, when flexure mechanisms are subjected to larger deflections, the guiding stiffness and load-bearing capacity decrease dramatically as a result of geometric non-linearities. Therefore, linear beam-deflection formulas loose accuracy and are no longer applicable [8, 30, 72 103 112].

This strong reduction in support stiffness is caused by coupling between compliant deformations in the degrees of freedom and stiff deformations in the support directions of the elastic elements, which occurs due to the change in geometry at large deflections. This can be illustrated by a simple leafspring for which the in-plane stiffness in undeflected condition is provided by the in-plane bending and/or shear stiffness, which is high. However, when the leafspring is deflected in the out-of-plane bending degree of freedom, the in-plane stiffness is mainly provided by the torsional compliance due to coupling between in-plane forces on the tip of the leafspring and bending moments (torsion) in the leafspring. This torsional compliance, which contributes to one of the degrees of freedom of a leafspring, inherently provides low resistance to deformations due to its long and slender shape [43, 72].

For moderate deflections of beam-shaped flexures (e.g. deflections up to about $10 \%$ of the length of the flexure) closed-form analytical models are available [4] [35 75]. However, these models are limited to $2 \mathrm{D}$ analysis or are only applicable to relatively simple flexure mechanisms, such as the parallelogram flexure mechanism or the cross-spring pivot, which are often unsuited for large range of motion applications due to their strong decrease in support stiffness when deflected. More complex geometries allow performance improvements for large range of motion applications [27 [108], which require numerical models to evaluate performance. However, due to the complex relation between the geometry of the flexures, the large deflections involved, and the resulting performance of the system, designing a "good" flexure mechanism suited for large stroke applications is far from trivial. 


\section{Optimization methods}

Complex mechanical design tasks, such as the design of a complex large stroke flexure mechanism, can be solved by utilizing numerical optimization algorithms. These algorithms can be used to search for the optimal material distribution given a specific design space, yielding maximum performance with respect to a specific design criteria. During the optimization process, the design parameters are iteratively improved by analyzing the performance of the current design and comparing it to previous solutions and constraints. This process is repeated until the solution has converged and no further improvements can be obtained. A schematic overview of a typical optimization process is provided in Fig. 1.1

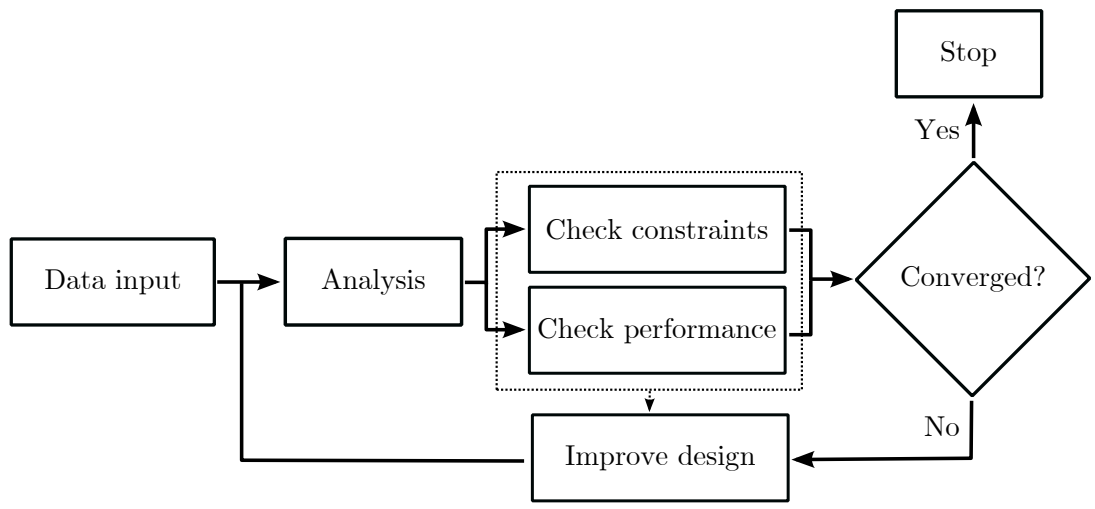

Figure 1.1: Flowchart of typical optimization process

Optimization strategies are often divided into three categories. Sorted by increasing degree of design freedom, these categories are named by "size", "shape" and "topology" optimization [28].

In size optimizations, the design domain is divided into a number of finite elements (e.g. one-dimensional truss or beam elements [20 21 46] or two-dimensional plate or shell elements [25 105]) of which cross-sectional properties or thickness are considered the optimization variables. Typical for size optimizations is the constant size of the numerical elements, resulting in a constant mesh size and fixed connectivity between the elements. Furthermore, by reducing an element's cross-sectional "size" to zero, individual elements can be effectively removed allowing for changes in topology. A schematic overview of a size optimization is provided in Fig. 1.2

Similarly to size optimizations, topology optimizations also divides the design domain into a number of finite elements. However, it differs in the dimension of 

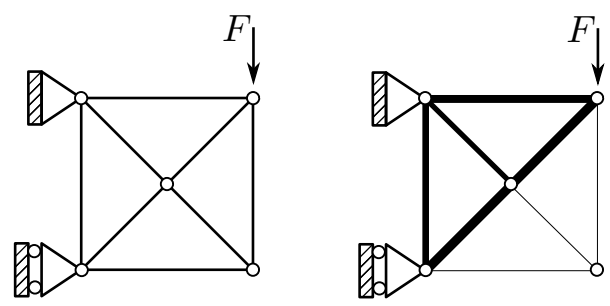

Figure 1.2: A schematic overview of a size optimization

the used numerical elements, which is equal to the dimension of the optimization problem (e.g. two-dimensional elements for a two-dimensional optimization problem). Therefore, the entire design domain can be discretized into a large number of finite elements. By employing piecewise constant "element densities" in each of the finite elements as the optimization variables [6 11] or by using level set functions [2, 102], the shape and topology of the structure can be optimized allowing for large design freedom. A schematic overview of a topology optimization is provided in Fig. 1.3
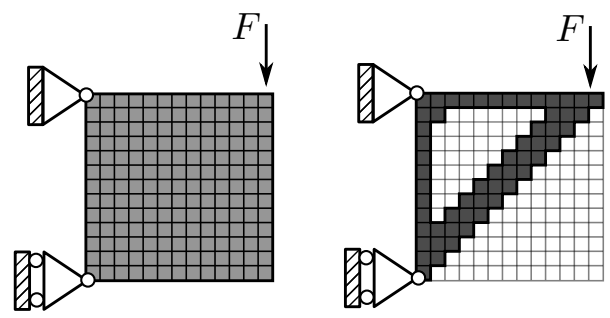

Figure 1.3: A schematic overview of a topology optimization

Both size and topology optimizations show good results for optimization problems which only concern small deformations of the structure for which linear approximations are sufficient. However, when larger deformations are considered, geometric non-linearities have to be included which can be computationally expensive. Moreover, the generally long and slender flexure shapes require a fine mesh, hampering the efficiency, especially for three-dimensional optimization problems. Furthermore, as no prior choice on the topology of the design is made, a large part of the design domain typically yields unusable numerical results when large deflections of the mechanism are required. This is a results of the large deformations resulting in a strongly distorted shape of the elements or void crushing, yielding high stress resultants, inaccurate results, difficulties with respect to convergence or even the absence of a static equilibrium [10, 76, 102]. Lastly, both size and topology optimizations consider a large number of optimiza- 
tion variables (typically equal to the number of elements), which complicates finding a good solution in the design space.

For shape optimizations, the shape of the structure is parameterized by a set of design variables which describe the geometry of the structure. A distinct difference with respect to topology optimizations is the absence of the ability to change the topology, which has to be conceived first. However, when optimizing flexure mechanisms designed for large range of motion applications, this can be used as an advantage. By using insight with respect to the degrees of freedom and constraints of elastic members, suitable topologies can be initiated allowing for large deflections in specific directions. As a result, these topologies can be deflected without strongly distorting the shape of the elements and without causing excessive stress resultants, resulting in a large portion of the design space providing useable numerical results. Furthermore, shape optimization methods can benefit from reduced complexity of the numerical model as the mesh of the structure can be chosen independently of the optimization variables, allowing for less elements or elements of a different type (e.g. one-dimensional beam elements for a three-dimensional optimization problem). Additionally, shape optimizations also allow for a small set of optimization variables to describe the relevant geometry, reducing the complexity of the optimization problem. A schematic overview of a shape optimization is provided in Fig. 1.4

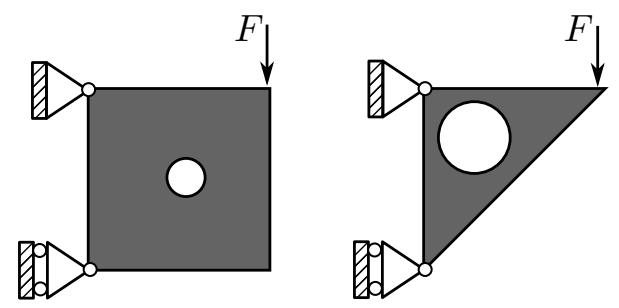

Figure 1.4: A schematic overview of a shape optimization

As a results of the inability of shape optimizations to change topology, the topology for the flexure mechanism has to be obtained separately. For this purpose, design principles for flexure-based systems and inspiration from existing flexurebased mechanism will be used in order to synthesize new designs.

\subsection{Research objectives}

The main research goal of this thesis is to develop flexure-based mechanisms with a large range of motion and increased performance to enable new preci- 
sion applications. To reach this goal, improved flexure-based joints are required which demand numerical optimization strategies to maximize performance.

Therefore, an optimization strategy has to be developed for optimizing the design of more complex flexure geometries which can deal with the large deformations involved. The purpose of this optimization method is to conceive and optimize the design of flexure geometries which were not feasible before, potentially leading to increased range of motion, load capacity and support stiffness.

The second goal is to use this optimization strategy to provide flexure-based equivalents for traditional sliding and rolling-element bearings, which can be used as flexure-based substitutes in existing or newly developed systems. For this purpose, three types of rotational joints are investigated in this research, consisting of the revolute joint (one rotational degree of freedom), the universal joint (two rotational degrees of freedom) and the spherical joint (three rotational degrees of freedom).

The final goal of this research is to demonstrate the applicability of large stroke flexure joints for flexure-based systems. Therefore, the development of a large range of motion flexure-based hexapod is pursued, targeting a large 6-DOF workspace combined with a high repeatability, accelerations and payload.

In summary, the goals of this research are

- The development of an efficient optimization strategy for complex large stroke flexure mechanisms.

- The development of large stroke flexure-based joints with one, two and three rotational degrees of freedom, the keystone elements for machine design.

- The design of a flexure-based six degree of freedom manipulator with large range of motion, utilizing the improved designs for the flexure-based rotational joints.

\subsection{Outline}

The first part of this thesis provides a description of an optimization strategy for flexure-based mechanisms, offering a general framework for the optimization of the flexure-based revolute, universal and spherical joint.

Chapter 2 describes this optimization method specifically developed for the optimization of flexure mechanisms subjected to large deformations. In this chapter, 
the developed method is tested on the optimization problem of a one degree-offreedom flexure-based revolute joint. By iteratively optimizing and improving the layout of the flexure hinge by using pre-defined building blocks, a new design for a flexure-based revolute joint with a range of motion of \pm 45 degrees is obtained.

To enable shape optimization of the universal and spherical joint which incorporate a spatial topology, collision of the flexures has to be included in the optimization strategy to prevent collision between the individual leafsprings. For this purpose, a collision detection method suited for large stroke flexure mechanisms is developed, which is described in Chapter 3 By taking advantage of this collision detection method, a new design and the optimization of a flexurebased spherical joint allowing for \pm 30 degrees tip-tilt motion and \pm 10 degrees of pan motion is presented in Chapter 4 By using a similar design strategy, a large stroke flexure-based universal joint is presented in Chapter 5

The second part of this thesis describes the design of a fully flexure-based hexapod robot, called the T-Flex. First, a custom design for a flexure-based suspension of an iron core torque motor is presented in Chapter 6 This suspension is designed to meet the high torque, repeatability and range of motion requirements for the hexapod. Next, a detailed description of the design and optimization of the hexapod is provided in Chapter 7. supplemented by experimental results of a prototype.

The chapters of this thesis are based on published scientific papers with minor adjustments and additional appendices. As each of these chapters has been written as a self-contained paper, the introductions of these chapters may present some overlap. 



\section{Design and optimization of large stroke flexure joints}





\title{
Chapter
}

\section{Building block-based spatial topology synthesis method for large-stroke flexure hinges}

\begin{abstract}
Large-stroke flexure mechanisms inherently lose stiffness in supporting directions when deflected. A systematic approach to synthesize such hinges is currently lacking. In this paper, a new building block-based spatial topology synthesis method is presented for optimizing large-stroke flexure hinges. This method consists of a layout variation strategy based on a building block approach combined with a shape optimization to obtain the optimal design tuned for a specific application. A derivative-free shape optimization method is adapted to include multiple system boundaries and constraints to optimize high complexity flexure mechanisms in a broad solution space. To obtain the optimal layout, three predefined threedimensional (3D) "building blocks" are proposed, which are consecutively combined to find the best layout with respect to specific design criteria. More specifically, this new method is used to optimize a flexure hinge aimed at maximizing the frequency of the first unwanted vibration mode. The optimized topology shows an increase in frequency of a factor ten with respect to the customary three flexure cross hinge (TFCH), which represents a huge improvement in performance. The numerically predicted natural frequencies and mode shapes have been verified experimentally.
\end{abstract}

\subsection{Introduction}

In high precision manipulators, often flexure-based mechanisms are used for their deterministic behavior due to the absence of friction, hysteresis, and back-

Published in: Journal of Mechanisms and Robotics (M. Naves, R.G.K.M. Aarts, D.M. Brouwer) 
lash [33, 39, 90, 92]. However, when designing flexure hinges, designers face a trade-off between flexibility for motion in certain desired directions and stiffness to constrain motion for guiding in the remaining directions [29]. Typical flexure hinges have a range of about 10 degrees beyond which the guiding stiffness and load-bearing capacity decrease dramatically [8, 30, 72, 103, 112]. A series chain of flexure hinges would increase the range of motion; however, this strategy creates underconstraints which need to be eliminated by adding extra constraints [38]. Consequently, it is far from trivial to design flexure hinges suited for large-stroke applications. In this paper, the goal is the development of a topology optimization suited for large deflection angles, with which the guiding stiffness can be greatly increased for flexure hinges vastly exceeding 10 degrees range of motion.

The advantages of optimizing the dimensions of large-stroke flexure hinges is already shown in previous studies [27,108]. For these shape optimizations, Wiersma described an optimization method which utilizes a parameterized flexible multibody model with nonlinear finite beam elements combined with a Nelder Mead algorithm to find the optimal shape. Furthermore, Markovic and Zelenika [57] described a finite element-based shape optimization, whereby the influence of variations in the design parameters on system performance is analyzed in order to find the optimal configuration. However, these methods restrict to optimizing the shape of a flexible mechanism, while the layout has to be conceived first. The required selection of the optimal layout can be done by performing numerous shape optimizations on a selection of layouts where afterward the best solution is selected. However, this search for the optimal layout can become rather cumbersome as a large number of layouts are available in literature [15], and the relation between layout, shape, constraints, and performance can become rather complex.

Systematic methods have been developed to perform structural topology optimizations. Frecker et al. [21] presented a ground/truss structure-based topology optimization, where a full ground structure (i.e., every node is connected to every other node by a truss element) is used to find the optimal topology by altering cross-sectional properties of each truss-element. Other typical structural topology optimizations are based on density distribution or level set-functions [2 3 6]. These methods divide the design domain into a large number of finite elements and employ piecewise constant "element densities" in each of the finite elements as the design variables. These methods show good results for two dimensional (2D) topology optimizations including small deformations. However, when more complex 3D topologies are considered, the design domain becomes very large, and topological optimizations can become computationally intensive. Finite element based topology optimizations may also suffer from difficulties in meshing 


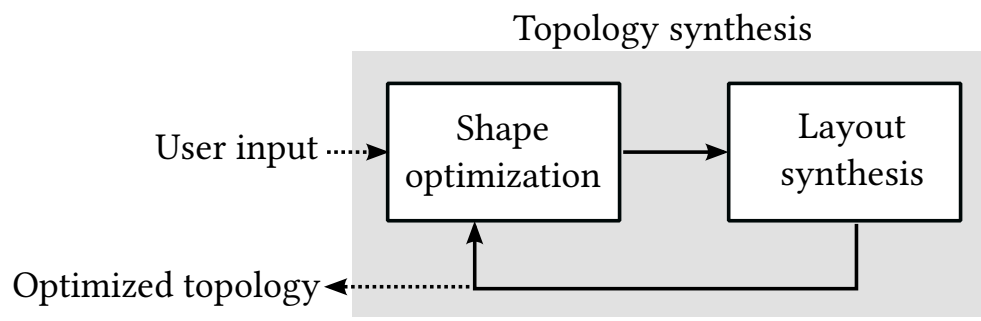

Figure 2.1: Schematic representation of a topology optimization based on a combined shape optimization and layout synthesis

parts with high aspect ratios like sheet flexures. Furthermore, geometrical nonlinearities are mostly disregarded [10]. This will reduce the accuracy of the finite element model and decreases robustness making them impractical for optimizing 3D large-stroke flexure mechanisms.

To reduce complexity, building block-based techniques have been developed which primarily rely on the characterization of the fundamental function of building blocks and formulating strategies to systematically combine them. A building block based method for the synthesis of a mechanism topology for any given compliance requirement is presented by Krishnan et al. [49]. However, this method does not incorporate an optimization strategy required to obtain the optimal topology and is again only applicable to 2D mechanisms with small deformations.

This paper presents a novel approach to optimize the spatial topology for largestroke flexure hinges. A key feature of this approach is an extension on the shape optimization method presented by Gunnink et al. [27] (section 2.2). This extension includes an adaptive objective function combined with a linear interpolation on the constraint functions to improve the optimization performance. Second, this adapted optimization method is merged with a novel 3D building block-based layout synthesis strategy to improve the layout (section 2.3. Third, a combination of this shape optimization and layout synthesis will effectively lead to a topology optimization as schematically illustrated in Fig. 2.1 Using the proposed topology optimization method, we present a flexure hinge with a unique 90 degrees range of motion with high support stiffness (section 2.4. 


\subsection{Shape optimization method}

The presented topology optimization strategy employs a shape optimization method and a layout synthesis strategy. In this section, the shape optimization method is discussed.

The Nelder-Mead based shape optimization presented by Gunnink et al. [27] is based on a parameterized model of a flexure mechanism, where the optimization objective is to find the optimal set of design parameters which maximizes performance. The Nelder-Mead algorithm [71] appears to be very suitable to find this optimal set as it delivers good robustness without the use of derivatives, which can be hard to obtain. By using this method, the number of required evaluations of the performance of the parameterized model is kept small, reducing the computational load. The performance of a specific set of design parameters is numerically evaluated with the flexible multibody program SPACAR [45, 59] which uses a series of interconnected nonlinear 3D finite beam elements which includes geometric nonlinearities. Flexibility of these elements is naturally included in the formulation owing to a specific choice of discrete deformation modes. Therefore, only a limited number of elements is required to produce fast and accurate results.

However, the optimization method proposed by Gunnink appears to have limited performance for optimizations in a large solution space with a large number of design variables and constraints. This is caused by the rejection of solutions which violate constraints, called unfeasible solutions, during subsequent iterations in order to maintain a feasible solution set. This rejection results in a performance degeneration of the Nelder-Mead algorithm. To prevent degeneration and to improve optimization performance, this method is extended with an adaptive objective function combined with a linear interpolation on the constraint functions to improve convergence to the optimum without the necessity of rejecting unfeasible solutions.

\subsubsection{Nelder-Mead method}

The suggested Nelder-Mead method searches for a minimum of an objective function $\mathcal{F}(\boldsymbol{p})$ with $N$ variables by comparing function values of $N+1$ parameter sets $\boldsymbol{p}$. Assume that the function values are sorted from small to large in $\boldsymbol{p}_{i}\left(\mathcal{F}\left(\boldsymbol{p}_{1}\right) \leq \mathcal{F}\left(\boldsymbol{p}_{2}\right) \leq \cdots \leq \mathcal{F}\left(\boldsymbol{p}_{N+1}\right)\right)$. With each iteration step, the parameter set with the highest function value $\left(\boldsymbol{p}_{N+1}\right)$ is replaced with a new parameter set 


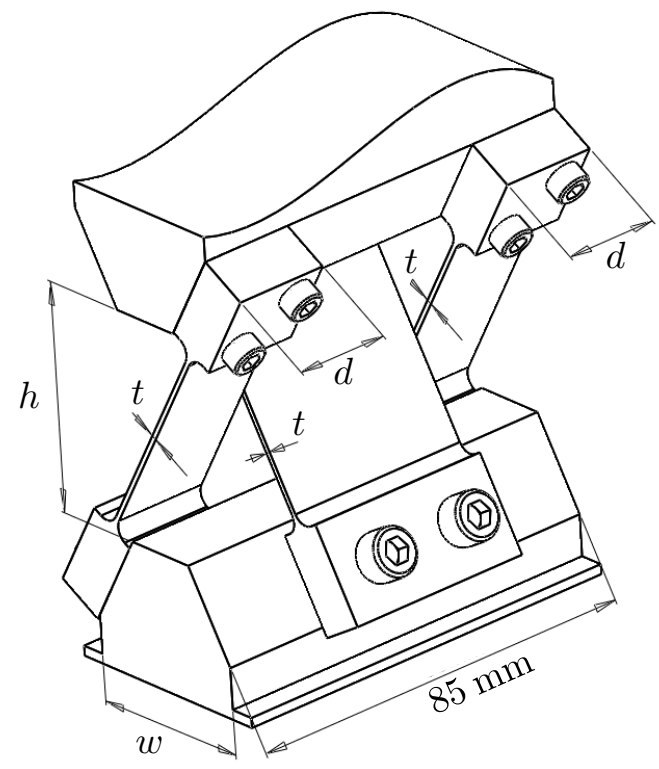

Figure 2.2: Parameterized model of a TFCH with design parameters $\boldsymbol{p}=[h, w, t, d]$ [27]

$\left(\boldsymbol{p}^{*}\right)$ according to

$$
\boldsymbol{p}^{*}=\boldsymbol{p}_{0}+\alpha\left(\boldsymbol{p}_{N+1}-\boldsymbol{p}_{0}\right)
$$

where $\boldsymbol{p}_{0}$ is the so-called centroid of the vertex spanned by $\boldsymbol{p}_{1}$ to $\boldsymbol{p}_{N}$, in example

$$
\boldsymbol{p}_{0}=\frac{1}{N} \sum_{i=1}^{N} \boldsymbol{p}_{\boldsymbol{i}}
$$

The value of $\alpha$ is determined by a series of logical steps and is bounded by $-0.5 \leq$ $\alpha \leq 2$ [22]. These steps are repeated until some level of convergence is obtained between the function values of all $N+1$ parameter sets.

\subsubsection{Adaptive objective function}

To perform the required shape optimization on a flexure mechanism, the performance of this mechanism has to be related to the shape by using the objective function $\mathcal{F}(\boldsymbol{p})$. For this purpose, a parameterized model of the considered flexure hinge is used. An example of a parameterized model of a simple TFCH with four design parameters is given in Fig. 2.2 
The objective is to find the optimal parameter set $\boldsymbol{p}$ which maximizes performance without violating constraints $(\mathcal{C}(\boldsymbol{p}) \leq 0)$. As the proposed Nelder-Mead method is a minimization algorithm, the optimization of the shape is given by

$$
\boldsymbol{p}_{\text {opt }}=\arg \min _{\boldsymbol{p}} \mathcal{F}(\boldsymbol{p}), \quad \text { subject to: } \quad \mathcal{C}(\boldsymbol{p}) \leq 0
$$

As optimization objective, the lowest frequency of the first unwanted vibration mode over the range of motion $([-\theta, \theta])$ is used as this value gives an indication of the support stiffness in load carrying directions. For evaluating this natural frequency $(f)$, a load case is defined by an inertia tensor and mass located at the pivot of the hinge which is equivalent to the load which has to be supported [108]. For some applications in which high support in a specific load carrying direction is required, using the stiffness as optimization objective could be a more intuitive approach. However, optimizing stiffness in a specific direction requires extra care as support stiffness in the remainder supportive directions could be strongly sacrificed in order to improve stiffness in the selected direction [27]. On the other hand, optimizing simultaneously for stiffness in multiple load carrying directions potentially requires an effective weighting between translational and rotational stiffness, which is not always trivial. By optimizing for the first unwanted vibration mode, stiffness in all directions are effectively taken into account and weighted with respect to the applied mass and inertia tensor, and evaluated based on the eigenvalues. The eigenmodes ensure that the worst-case eigenfrequency is used as an optimization objective.

Different from the original algorithm, convergence from unfeasible to feasible solutions is not enforced with a "hard" constraint by rejecting unfeasible solutions, but instead an adapted objective function is introduced which adds a performance "penalty" to unfeasible solutions when a constraint is violated according to

$$
\mathcal{F}(\boldsymbol{p})=\lambda^{3} \min _{\theta}(f(\boldsymbol{p}, \theta))^{-1},
$$

with

$$
\lambda= \begin{cases}1 & \text { if } \quad \mathcal{C}(\boldsymbol{p}) \leq 0 \\ 1+\mathcal{C}(\boldsymbol{p}) & \text { if } \quad \mathcal{C}(\boldsymbol{p})>0\end{cases}
$$

With this penalty, a "soft" constraint is introduced which enforces convergence to a feasible solution and prevents the necessity of rejecting unfeasible solutions. Furthermore, it enables the use of any initial parameter set at the start of the optimization, disregarding its feasibility. Hereby, an extensive initial search to obtain an initial feasible set of solutions is avoided, and the computational load is reduced. 
For the penalty function of Eq. (2.4), a third-order penalty is suggested as this penalizes unfeasibility such that a less feasible solution is always less favorable, and convergence to a more feasible solution is maintained. A higher order penalty could also be used, but is not required and could introduce numerical problems. A typical constraint function $\mathcal{C}$ to bound the maximum stress $(\sigma)$ can be written as

$$
\mathcal{C}(\boldsymbol{p})=\max _{\theta}\left(\frac{\sigma(\boldsymbol{p}, \theta)-\sigma_{\max }}{\sigma_{\max }}\right)
$$

The penalty function of Eq. 2.5 can be expanded to include multiple constraint functions, for example, a constraint on the maximum actuation moment or the minimal buckling load.

\subsubsection{Constraint interpolation}

In the previous section, a soft constraint is already introduced with the described penalty function according to Eq. 2.5 in order to enhance convergence to a feasible solution. However, to further improve the optimization performance, a linear interpolation on the constraint functions is added.

Quite often optimal solutions are located close to or at the edge of feasibility, resulting in a large number of unfeasible solutions during convergence to the optimum. If the centroid of the simplex is feasible $\left(\mathcal{C}\left(\boldsymbol{p}_{0}\right) \leq 0\right)$ and an unfeasible parameter set is generated $\left(\mathcal{C}\left(\boldsymbol{p}^{*}\right)>0\right)$, it can be stated that in-between those parameter sets a constraint is exceeded. With a linear interpolation, an estimation can be made of the parameter set which is close to the edge of feasibility and likely near the optimum. This interpolation is given by

$$
\begin{gathered}
\text { if } \quad \mathcal{C}\left(\boldsymbol{p}^{*}\right)>0 \quad \text { and } \quad \mathcal{C}\left(\boldsymbol{p}_{0}\right) \leq 0, \\
\boldsymbol{p}^{* *}=\boldsymbol{p}_{0}+\mathcal{C}\left(\boldsymbol{p}_{0}\right) \frac{\boldsymbol{p}^{*}-\boldsymbol{p}_{0}}{\mathcal{C}\left(\boldsymbol{p}_{0}\right)-\mathcal{C}\left(\boldsymbol{p}^{*}\right)}
\end{gathered}
$$

where the constraint function of the centroid can be estimated by

$$
\mathcal{C}\left(\boldsymbol{p}_{0}\right) \approx \frac{1}{N} \sum_{i=1}^{N} \mathcal{C}\left(\boldsymbol{p}_{i}\right)
$$

to avoid extra objective function evaluations.

By using this linear interpolation, the "soft" constraint in the objective function is complemented with an interpolation strategy on the constraints when the centroid of the simplex has become feasible, improving convergence. However, as the linear interpolation gives an estimate of the location of the edge of feasibility, 
Table 2.1: Boundary conditions for testing the shape optimization corresponding to the design parameters of the TFCH in Fig. 2.2

\begin{tabular}{lll} 
Design parameter & Lower bound & Upper bound \\
\hline \hline$t$ & $0.2 \mathrm{~mm}$ & $1 \mathrm{~mm}$ \\
$h$ & $5 \mathrm{~mm}$ & $100 \mathrm{~mm}$ \\
$w$ & $5 \mathrm{~mm}$ & $100 \mathrm{~mm}$ \\
$d$ & $5 \mathrm{~mm}$ & $40 \mathrm{~mm}$ \\
\hline
\end{tabular}

the corrected parameter set $\boldsymbol{p}^{* *}$ is not necessarily feasible. Therefore, the soft constraint from the penalty function of Eq. 2.5) is maintained, even when the interpolation is applied according to Eq. 2.7.

\subsubsection{Shape optimization performance}

To test the performance of the described shape optimizer, 50 shape optimizations have been performed on the parameterized TFCH of Fig. 2.2 starting from randomly generated initial parameter sets. The best solution of the complete set is considered the global optimum and is used to evaluate the probability of finding a solution close to optimal when considering the broad search space according to Table 2.1

Furthermore, each shape optimization is terminated when the performance of the parameter sets have been converged to a certain threshold. This threshold is defined as

$$
\frac{\mathcal{F}\left(\boldsymbol{p}_{1}\right)}{\mathcal{F}\left(\boldsymbol{p}_{N+1}\right)}>0.995
$$

which corresponds to a deviation in performance of less than $0.5 \%$ between the parameter sets. The remaining optimization parameters are shown at Table 2.2 and will be discussed in section 2.4.1

In Table 2.3 an overview of the shape optimization performance is presented based on the conducted set of shape optimizations. At average, only 135 objective function evaluations had to be performed to obtain the desired convergence. As a large number of local optimums are available in the considered solution space, each individual optimization only searches for a single optimum (either local or global), and the probability of finding the global optimum is not equal to $100 \%$. By performing a larger set of shape optimizations with different initial 
Table 2.2: Optimization parameters corresponding to the proposed mechanism by Folkersma et al. [19]

\begin{tabular}{ll} 
Parameter & Value \\
\hline \hline$\theta_{\min } / \theta_{\max }$ & $-45 \mathrm{degrees} / 45$ degrees \\
$\sigma_{\max }$ & $600 \mathrm{MPa}$ \\
$E$ & $210 \mathrm{GPa}$ \\
$G$ & $80 \mathrm{GPa}$ \\
$m_{\text {load }}$ & $0.574 \mathrm{~kg}$ \\
$I_{x x}$ & $3.760 \cdot 10^{-3} \mathrm{~kg} \mathrm{~m}^{2}$ \\
$I_{y y}$ & $3.528 \cdot 10^{-2} \mathrm{~kg} \mathrm{~m}^{2}$ \\
$I_{z z}$ & $3.826 \cdot 10^{-2} \mathrm{~kg} \mathrm{~m}^{2}$ \\
$d_{\max }$ & $85 \mathrm{~mm}$ \\
\hline
\end{tabular}

parameter sets, the probability of finding a solution close to the global optimum can be increased. For example, according to Table 2.3 the probability of not obtaining a solution within $5 \%$ of the global optimum in a single optimization run equals to $100 \%-42 \%=58 \%$. When ten shape optimizations are combined, this probability reduces to $0.58^{10} \cdot 100 \%=0.43 \%$. As a single optimization run can be conducted within a minute by a modern computer system, and as multiple optimizations can be performed in parallel on a multicore processor, a satisfactory solution can be obtained rapidly.

When considering more complex shape optimizations with more design variables, typically, the probability of finding the global optimum decreases, and the number of required objective function evaluations increases. Furthermore, the required computational time for evaluating the objective function will typically increase as the complexity of the SPACAR model increases. However, a satisfactory convergence to the global optimum is maintained, and a close to optimal solution for more complex flexure hinges can typically be obtained within a few hours. 
Table 2.3: Average shape optimization performance of a single optimization run based on 50 separate optimizations of a TFCH

No. of objective function evaluations

Probability of obtaining performance within $1 \%$ of the global optimum $24 \%$

Probability of obtaining performance within 5\% of the global optimum $42 \%$

\subsection{Layout synthesis}

In order to optimize the topology as illustrated in Fig. 2.1, a systematic approach is required to synthesize the layout for large-stroke flexure hinges. For obtaining this optimal flexure layout, a number of compliant 3D "building blocks" are defined. The most basic layout for a flexure hinge, which is capable of obtaining a large deflection angle with an acceptable level of performance, is the customary TFCH [31 108], consisting of three leafsprings. This layout will be used as initial reference from where layout is synthesized.

For this approach, the flexure hinge is divided into two separate sections: the inner and outer building blocks as illustrated in Fig. 2.3. where both outer blocks are taken identical. The $z$-axis is defined as the desired axis of rotation, and a corotational coordinate frame is considered which is aligned with the end effector. Furthermore, the directional support stiffness is defined as the resistance to deformation in a specific direction, while motion in all other directions is constrained. This definition of the support stiffness is used in order to clearly identify the typical stiffness characteristics of the building blocks. Furthermore, when multiple building-blocks are used to assemble a flexure hinge, motion in the other directions is typically constrained by the remainder building blocks, justifying this assumption.

\subsubsection{Building blocks}

Conducted from the FACT method, each compliant element of each building block should only provide degrees of constraint which intersect the axis of rotation (the instant center of rotation) or which are aligned parallel to this axis of rotation (intersects at infinity) in order to obtain a mechanism which allows a single rotational degree of freedom [36]. To allow a large deflection angle (defined as the rotation of the end effector in the degree of freedom), this criteria should be satisfied over the entire range of motion. 


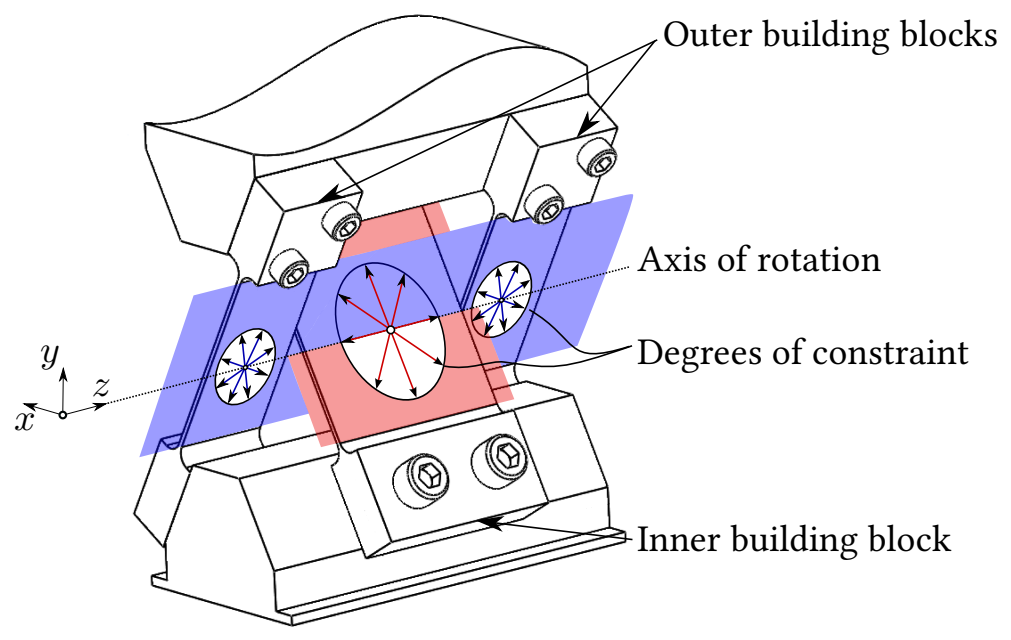

Figure 2.3: Visualization of the planes spanned by the degrees of constraint of the outer and inner building blocks of a TFCH

However, for larger deflection angles, the typical degrees of constraint will shift due to deformation of the flexures. To ensure all degrees of constraint are intersecting the axis of rotation over the full range of motion, all degrees of constraint not parallel to the axis of rotation should span exactly two planes when considering large deflection angles. This results in an instant center of rotation exactly at the intersecting line of both planes, whereby each degree of constraint automatically intersects the axis of rotation (Fig. 2.3. When more than two planes are spanned by the degrees of constraint, typically multiple intersecting lines of the degrees of constraint can be identified due to the nonlinear shortening of the flexures.

To illustrate this phenomena, the location of the instant center of rotation of a cross hinge consisting of two and three flexures is compared. Consider cross hinge $A$ consisting of three flexures $(a, b, c)$, where flexure $a$ is removed to form cross hinge $B$, and flexure $c$ is removed to form cross hinge $C$ (Fig. 2.4 . For cross hinges $B$ and $C$, the instant center of rotation in deflected state is given by the solid cross-hair, which has an unique position for each of the hinges. As the instant center of rotation of flexure $a$ and $b$, respectively, $b$ and $c$ are not in the same position, the combined instant center of rotation for flexure $a, b$, and $c$ (cross hinge $A$ ) will trigger a reduced "quality" [4] of the intended degree of freedom which strongly increases stress and jeopardizes compliance in the desired motion direction. 
$-\phi$ - Instant center of rotation

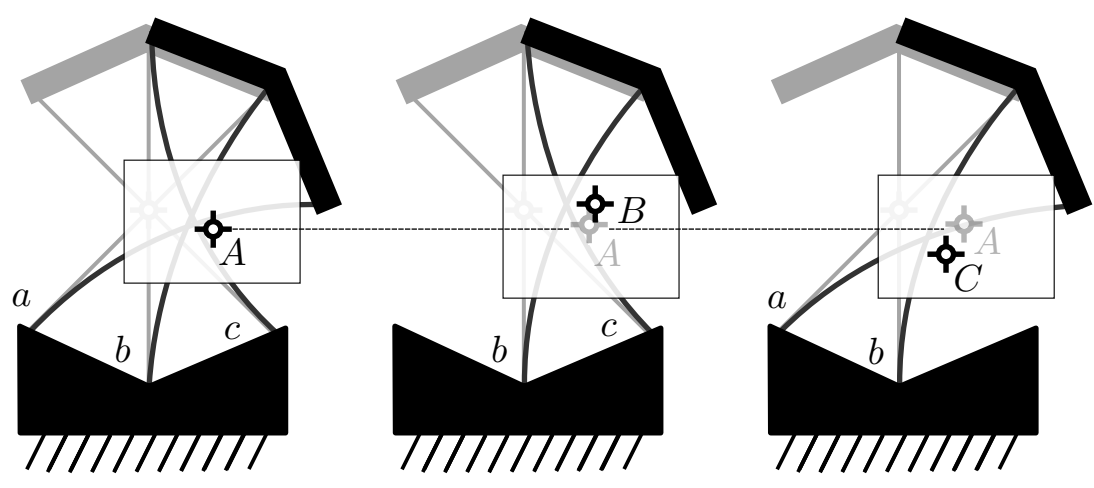

$A=B+C$

$B$

C

Figure 2.4: Calculated instant center of rotation of a cross hinge consisting of two and three flexures

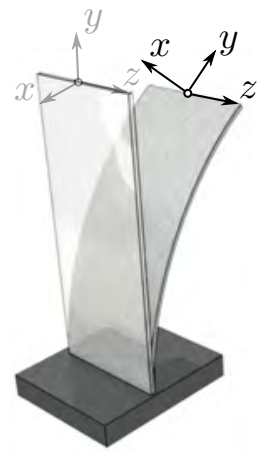

(a)

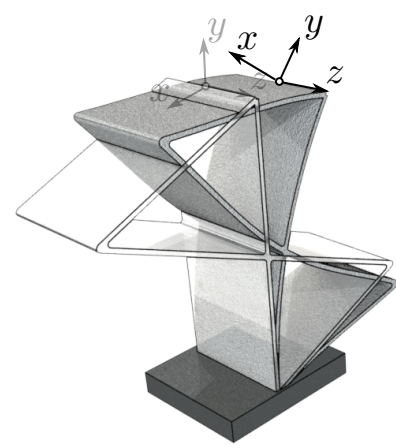

(b)

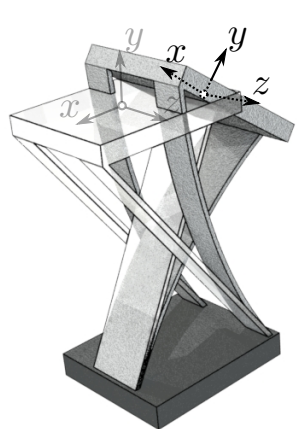

(c)

Figure 2.5: Flexural building blocks used to "synthesize" flexure layout: (a) LS: leafspring, (b) TRLS: torsionally reinforced leafspring, and (c) TFCH: three flexure cross hinge

As multiple building blocks are combined at either the outer and inner building block position, where both outer building blocks are taken identical, all degrees of constraint of each building block should span only a single plane to avoid this overconstraint configuration for large deflection angles. 


\section{Building block 1: Leafspring}

The first building block considered is a single leafspring (LS, Fig. 2.5a), which is commonly used in flexure mechanisms. Because a leafspring provides only degrees of constraint in-plane of the leafspring, only a single plane is spanned by the degrees of constraint. This element typically has only limited support when considering the stiffness properties in deformed state, except for translational stiffness in $z$-direction. However, it does have good motion compliance in the desired degree of freedom ( $z$-rotation).

\section{Building block 2: Torsionally reinforced leafspring}

To improve torsional stiffness around the $y$-axis and in-plane bending stiffness around the $x$-axis, the so-called torsionally reinforced leafspring (TRLS, Fig. 2.5b is presented, which is inspired on the infinity hinge [108]. This building block consists of a single central leafspring reinforced with one or more folded flexures to mainly improve torsional and in-plane bending stiffness (rotational stiffness around the $x$ - and $y$-axis). As each folded flexure only adds a degree of constraint parallel to the axis of rotation, all degrees of constraint not parallel to the axis of rotation still span a single plane. The extra folded flexure provides an increase in support stiffness while slightly decreasing the motion compliance with respect to the degree of freedom. Translational stiffness in $x$ - and $y$-directions remains limited.

\section{Building block 3: Double three flexure cross hinge}

The third building block, which aims at increasing translational stiffness in the $x$ - and $y$-directions over the range of motion, is a TFCH, Fig. 2.5c As a single TFCH as a separate building block would violate the criterium of all degrees of constraint to span a single plane (the inner and outer leafsprings each spans a separate plane), two TFCH's are stacked in series to form the so-called double TFCH. As illustrated in Fig. 2.6 with a pseudo rigid body model, two TFCH's in series are equivalent to a three bar linkage with two rotational joints. The effective degrees of constraint of this three bar linkage spans a single plane which intersect both joints.

\subsubsection{Building block overview}

An overview of the typical stiffness properties at deflected state of each "building block" is given in Table 2.4 For this table, rotational stiffness around the $z$-axis is not considered as this corresponds to the stiffness in the intended degree of freedom. Numerical values of the directional support stiffness (defined as the 


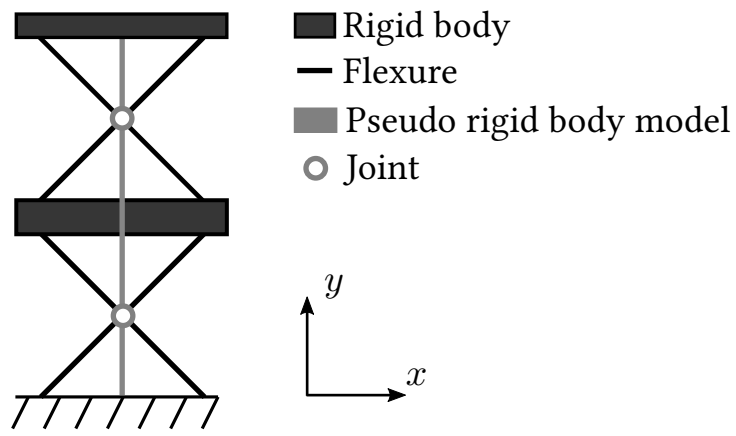

Figure 2.6: Pseudo rigid body representation of a double TFCH in series

Table 2.4: Typical building block support stiffness in the deformed state with respect to the corotational coordinate system as indicated in Fig. 2.5

\begin{tabular}{llll} 
Support stiffness & LS & TRLS & double-TFCH \\
\hline \hline$X$-translation $\left(10^{5} \mathrm{~N} / \mathrm{m}\right)$ & $-(0.26)$ & $-(0.40)$ & $+(2.5)$ \\
$Y$-translation $\left(10^{5} \mathrm{~N} / \mathrm{m}\right)$ & $-(6.4)$ & $-(7.0)$ & $+(50)$ \\
$Z$-translation $\left(10^{5} \mathrm{~N} / \mathrm{m}\right)$ & $+(20)$ & $+(20)$ & $-(4.6)$ \\
$X$-rotation $\left(10^{2} \mathrm{Nm} / \mathrm{rad}\right)$ & $-(0.98)$ & $+(4.1)$ & $-(2.0)$ \\
$Y$-rotation $\left(10^{2} \mathrm{Nm} / \mathrm{rad}\right)$ & $-(0.13)$ & $+(5.3)$ & $-(1.6)$ \\
\hline
\end{tabular}

resistance to deformation in a specific direction while motion in all other directions is constrained) for some typical "building block" dimensions with a width of $20 \mathrm{~mm}$, height of $50 \mathrm{~mm}$, flexure thickness of $0.5 \mathrm{~mm}$, E-modulus of $210 \mathrm{GPa}$, and an deflection angle of 25 degrees are given between parentheses. Note that these values are affected by the chosen geometry and material properties. However, they do provide an indication of the stiffness characteristics of each building block with respect to each other.

\subsubsection{Layout update strategy}

To obtain the optimal combination of the previously described building blocks, a layout update strategy is developed. This strategy starts with the shape optimization of the initial reference layout, which consists of three single leafsprings (a TFCH). 
After the initial shape optimization, the first unwanted vibration mode is examined, and the critical stiffness associated with this mode is determined. Next, the critical support stiffness is possibly improved by using a building block with increased support stiffness in the desired direction according to Table 2.4 This layout update is followed by a new shape optimization to re-obtain the optimal shape for the new layout. By repeating this process of subsequent shape optimizations and layout updates, the optimal layout is tried to be found. Three cases can be identified for alternating the layout:

- If the rotational stiffness around the $x$ - or $y$-axis is critical, a folded flexure should be added to the inner central leafspring (to form a TRLS). The TRLS should be placed at the inner flexure position as maximum width is required to maximize the rotational stiffness around the $x$ - and $y$-axis (especially when considering the constraint warping phenomenon [103 108] causing torsional stiffening for wide and short flexures).

- If translational stiffness in the $x$ - or $y$-direction is critical, a double TFCH should be used. This double TFCH should initially be positioned at the outer flexure positions to reserve the inner position for a TRLS. If the translational stiffness remains critical, a double TFCH can also be placed at the inner flexure position.

- In case translational stiffness in $z$-direction is critical, the leafspring typically provides the best support stiffness (in combination with high compliance in the degree of freedom).

A complete overview of this optimization strategy is given in Fig. 2.7. which can be used to systematically optimize the topology for flexure hinges taking into account large deflection angles. 


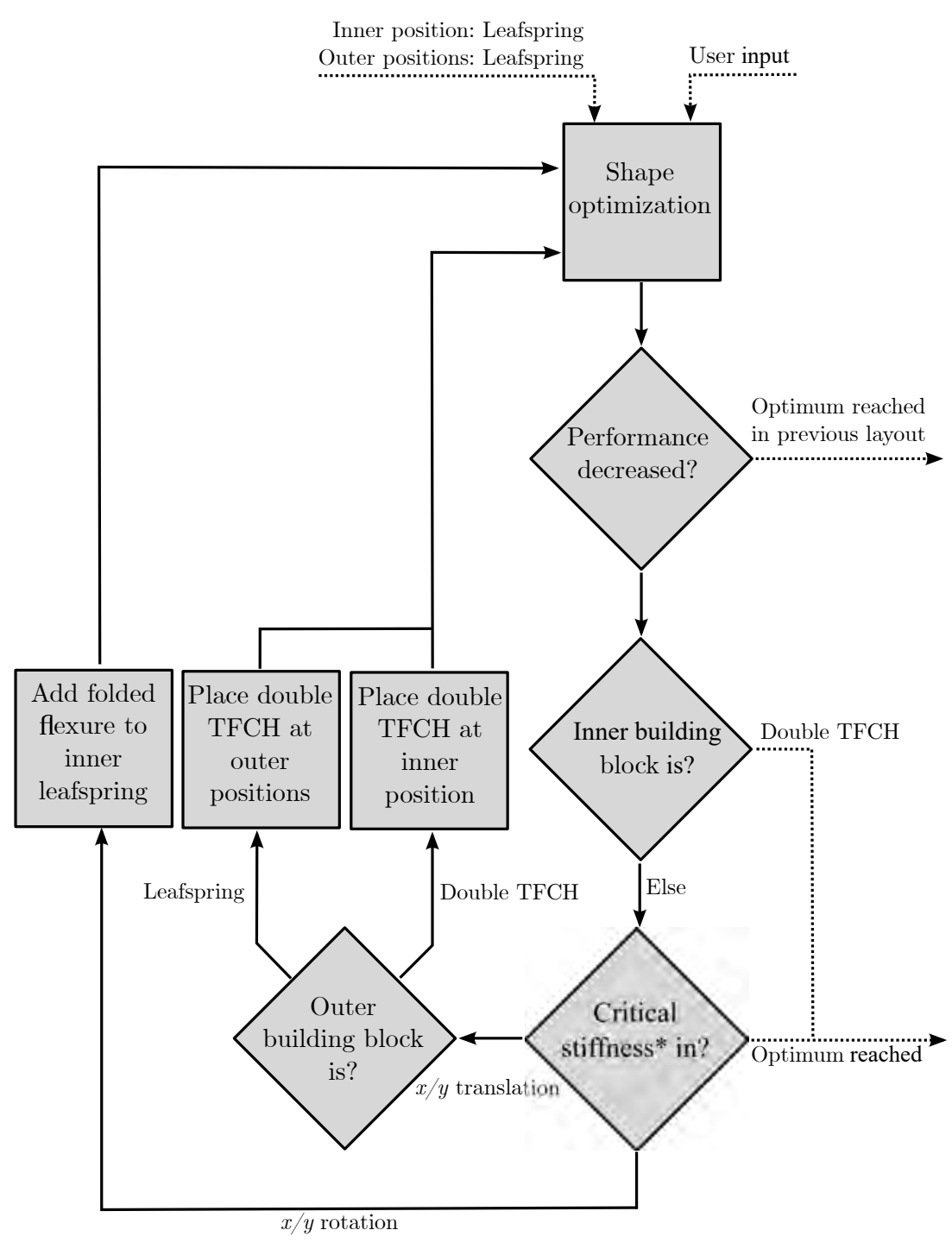

* Or the stiffness which contributes primarily to the critical vibration mode

Figure 2.7: Topology optimization based on a combined shape optimization and layout synthesis 


\subsection{Results}

In this section, a topology optimization example is given of a flexure hinge tuned for a specific application to show the potential of the presented method. As a test case, the mechanism presented by Folkersma et al. [19] will be used with the associated load case derived by Wiersma et al. [108].

\subsubsection{Optimization case}

In short, the hinge for the selected application is subjected to a mixed load of inertia $\left(I_{x x}, I_{y y}, I_{z z}\right)$ and mass $\left(m_{\text {load }}\right)$ where most emphasis is put in the inertial load around the $y$-axis. Another optimization case, considering the optimization of the off-axis support stiffness, is presented in Appendix 2.7.1

An overview of the complete set of input parameters is given in Table 2.2 Goal is to achieve a maximum natural frequency of the first unwanted vibration mode to maximize position accuracy of the system. The total stroke is set at $\theta_{\min }=-45$ to $\theta_{\max }=45$ degrees deflection, and the maximum width $\left(d_{\max }\right)$ of the hinge is bounded to $85 \mathrm{~mm}$, illustrated in Fig. 2.2 The selected material is steel, where we limit the allowable stress due to deformation $\left(\sigma_{\max }\right)$ to $600 \mathrm{MPa}$. The layout and performance (defined as the lowest value of the frequency of the first unwanted vibration mode over the range of motion) of each intermediate shape optimized layout is given in Table 2.5 with the results visualized in Appendix 2.7.2 Furthermore, the performance versus deflection angle is plotted in Fig. 2.8 For this example, folded flexures are added in increments of two to accelerate the optimization procedure. For optimizing the shape at each intermediate layout, ten shape optimizations are conducted to ensure a solution close to the global optimum (total of 50 shape optimizations for five iterations in updating the layout).

The initial layout consisting of three leafsprings shows a minimum natural frequency of $11 \mathrm{~Hz}$ over its range of motion. With each layout update, an improvement in performance is observed. The optimal layout is obtained when six folded flexures have been added to the inner leafspring and double TFCH's have been placed at the outer building block positions. To further improve performance, more folded flexures at the inner flexure position would be required. However, due to collision between folded flexures when deflected, design freedom of the folded flexures decreases, limiting overall performance when more folded flexures are added. 
Table 2.5: Performance of the optimized shape for each intermediate layout. The number of folded flexures for the TRLS is indicated between parentheses

\begin{tabular}{llll} 
Iteration & Outer flexures & Inner flexure & Performance $(\mathrm{Hz})$ \\
\hline \hline 1 & Leafspring & Leafspring & 11 \\
2 & Leafspring & TRLS (2x) & 42 \\
3 & Double TFCH & TRLS (2x) & 49 \\
4 & Double TFCH & TRLS (4x) & 81 \\
5 & Double TFCH & TRLS (6x) & 102 \\
\hline
\end{tabular}

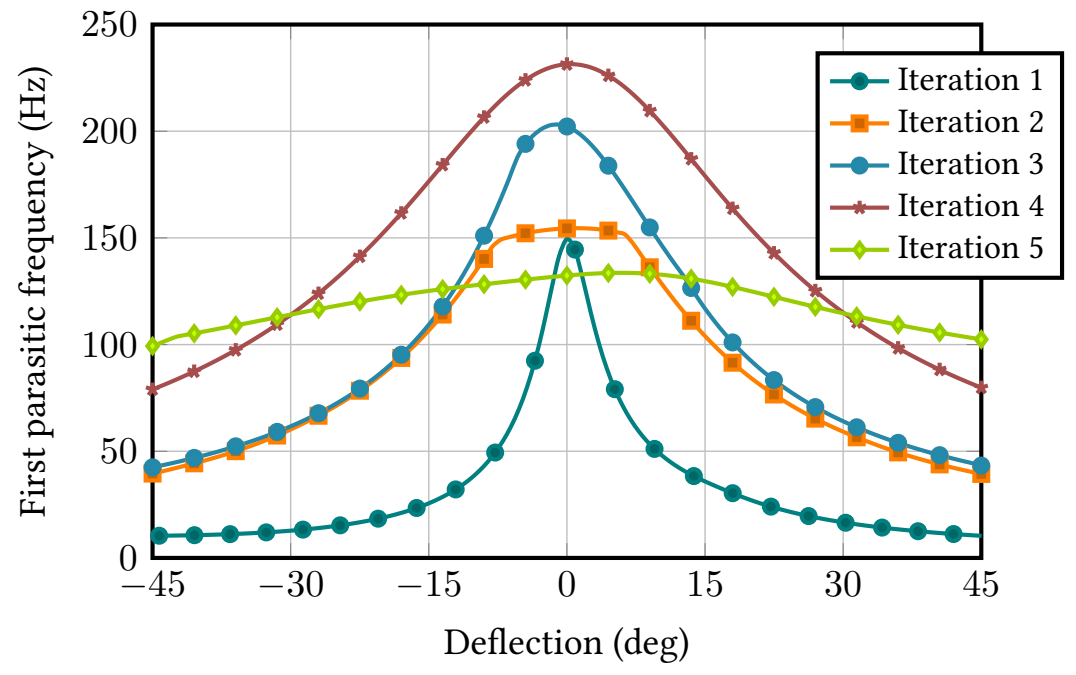

Figure 2.8: Natural frequencies of the first unwanted vibration mode of intermediate shape optimized layouts 


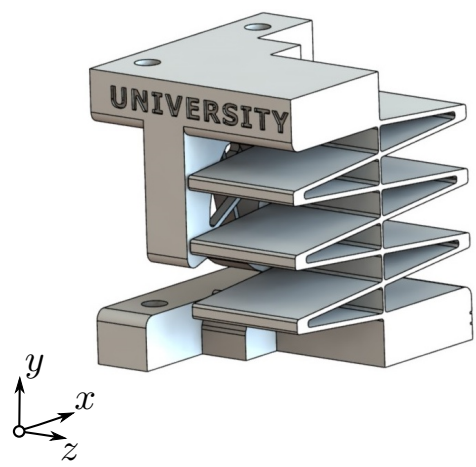

(a)

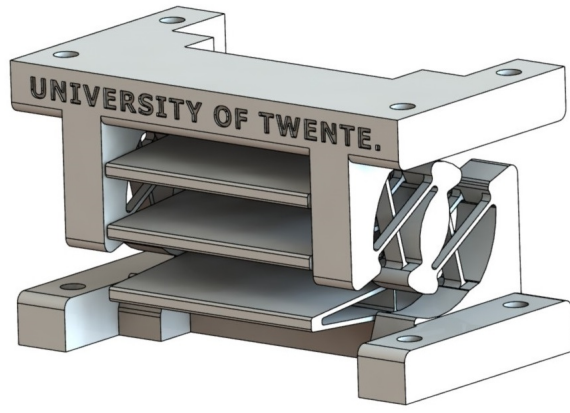

(b)

Figure 2.9: $\mathrm{CAD}$ rendering of the manufactured flexure mechanism existing of a TRLS (3x) supported by two double TFCH's: (a) section view and (b) complete view

From the performance versus deflection plot in Fig. 2.8 it can be concluded that the performance at small deflection angles is sacrificed in order to maximize performance at larger deflections. This is directly caused by the optimization criteria, which is defined as a maximization of the lowest natural frequency of the first unwanted vibration mode over the entire range of motion. On the other hand, however, the minimum natural frequency has increased with almost a factor of ten with respect to the initial layout, which is a major improvement in performance.

\subsubsection{Experimental validation}

To validate the SPACAR models which are used for evaluating the objective and constraint functions, an experimental validation is conducted. The optimized topology is used to design a flexure hinge, which, for practical reasons, is made of nylon and manufactured with selective laser sintering (SLS). Because of the different material properties of nylon with respect to steel and due to geometrical limitations with respect to the sintering process of nylon, the flexure thickness is increased to comply with the SLS nylon additive manufacturing technology. A rendering of the manufactured flexure hinge is given in Fig. 2.9 Furthermore, for experimental reasons, a slightly modified load case has been used $(m=0.47 \mathrm{~kg}$, $I_{x x}=4.7 \cdot 10^{-4} \mathrm{kgm}^{2}, I_{y y}=3.83 \cdot 10^{-3} \mathrm{kgm}^{2}$ and $\left.I_{z z}=4.08 \cdot 10^{-3} \mathrm{kgm}^{2}\right)$.

To verify the results, a series of measurements are performed to confirm the nat- 


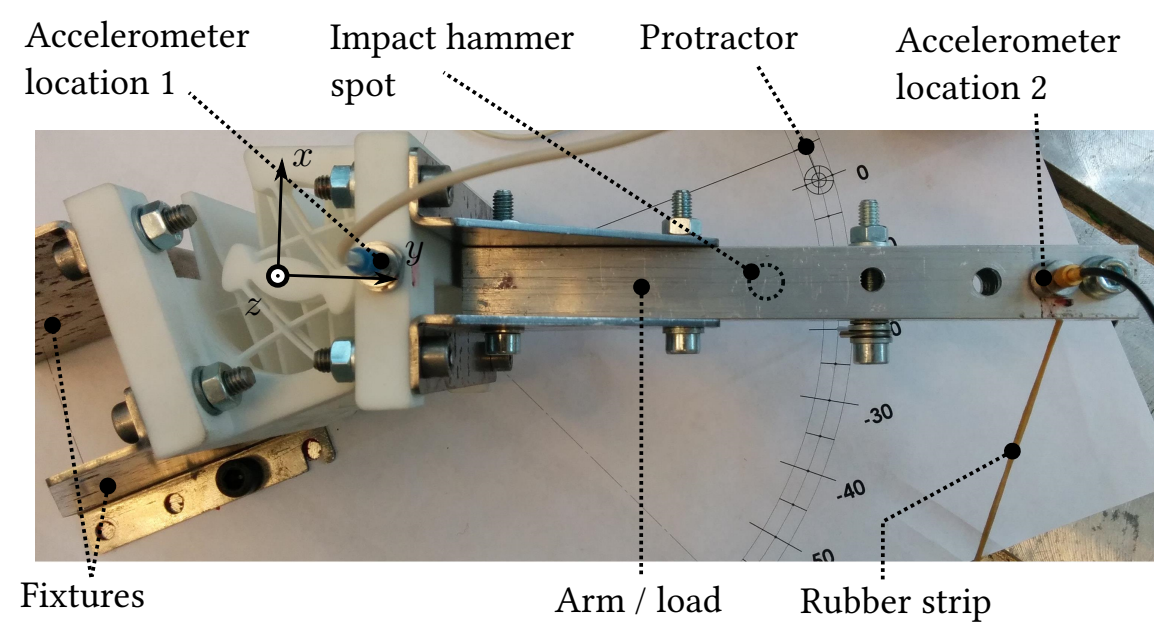

Figure 2.10: Measurement setup for testing rot- $x$ vibration modes at -20 degrees deflection

ural frequencies of the vibration modes of interests. Those modes are rotational modes around the $x$ - and $y$-axis (rot- $x$ and rot- $y$ ) and translational modes in the $x$ - and $y$-directions (trans- $x$ and trans- $y$ ). In Fig. 2.10 the measurement setup is shown for measuring rotational modes around the $x$-axis. Two accelerometers are aligned in $z$-direction, and an impact hammer is used in the same direction. By using the FRF data from impact to accelerations, the natural frequency of the vibration mode associated with $z$-motions could be identified. To measure the natural frequency over the entire range of motion, the hinge is held in deformed state by an elastic element to prevent any interaction with the considered vibrational modes. Those measurements are repeated over the entire range of motion in steps of 5 degrees, where a protractor is used to obtain the current deflection angle. To identify all modes of interest, similar measurements in $x$ and $y$-directions are performed with different sensor orientations. The rotational mode around the $y$-axis can be identified by all accelerometers as this mode is accompanied with small translational motions in each direction, so no separate experiment had to be conducted for this specific mode.

An example of the frequency response from impact to accelerations in $x-, y-$, and $z$-directions at only the second accelerometer location is provided in Fig.2.11. The rotational $x$-mode (associated with $z$-accelerations) can be clearly identified at $37 \mathrm{~Hz}$, other modes associated with $x$ - and $y$-accelerations can be identified at higher frequencies. When measurements are performed at larger deflection 


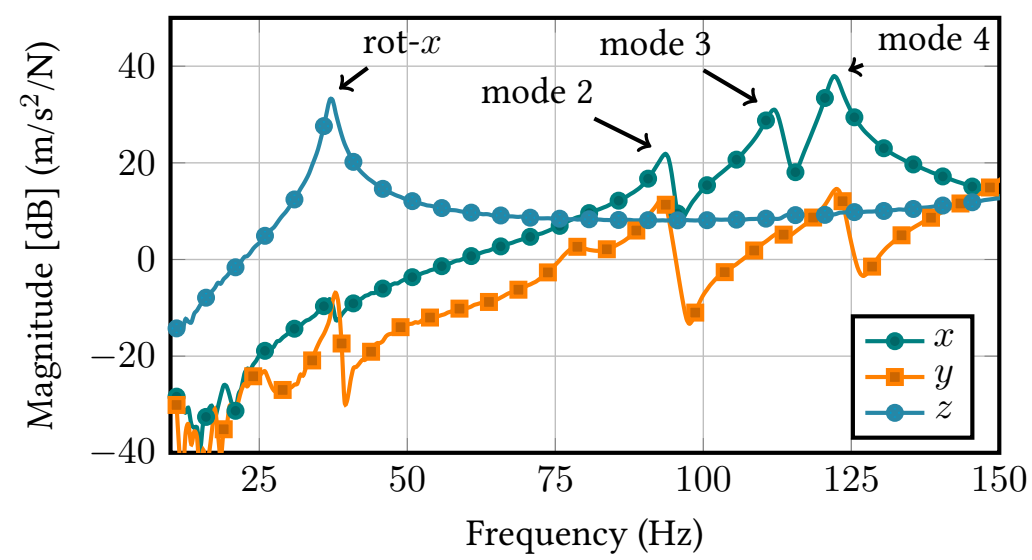

Figure 2.11: Frequency response from impact to $x$-, $y$ - and $z$-accelerations at accelerometer location 2 and 0 degrees deflection

angles, modes corresponding to $x / y$-translations can be identified less clear as the frequencies are close to each other and interaction between eigenmodes occurs. However, the resonance frequencies can still be identified.

An overview of the experimental results over the entire range of motion is given in Fig. 2.12 The frequency of the first vibrational mode, consisting of a rotation around the $x$-axis, shows a good agreement with the model results. The second and third vibrational modes, consisting of translations in the $x$ - and $y$-directions and the fourth vibration mode consisting of a rotation around the $y$-axis, shows a slight deviation in frequency. However, these deviations are in an acceptable range and only provide a positive increase in frequency. The deviations with respect to the experimental results can be explained by interaction between modes with frequencies close to each other and mixing of eigenmodes. Furthermore, possible inconsistencies in material properties and flexure thickness due to the sintering process can be of influence. The overall trend of the natural frequencies of the vibration modes shows good agreement and confirms the used models, although the exact mode shape could only be confirmed for the first vibrational mode. 


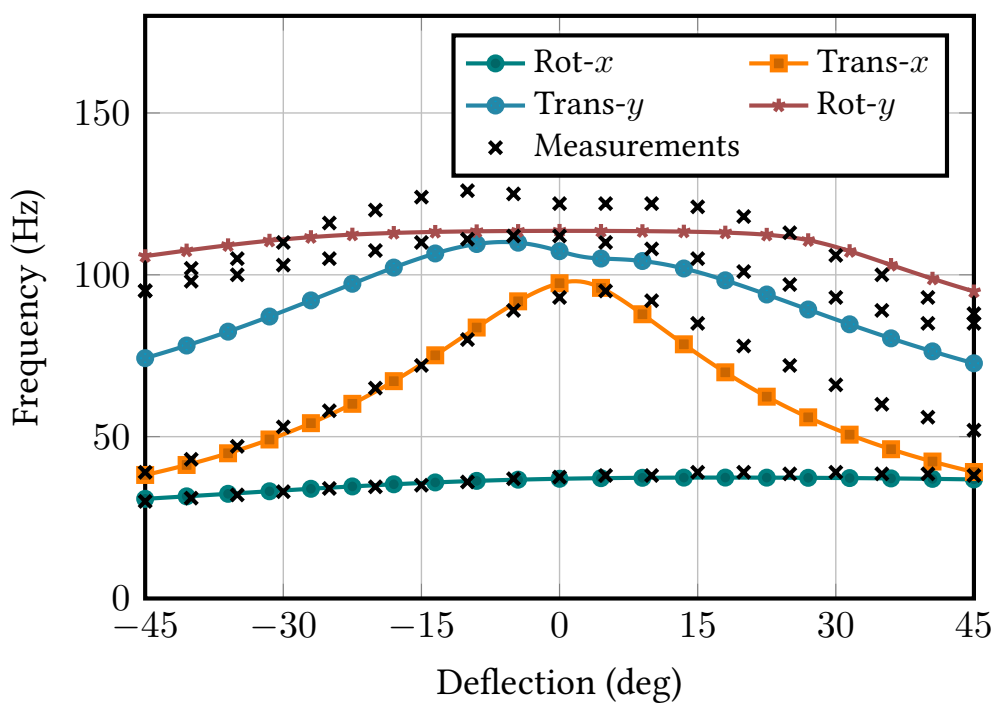

Figure 2.12: Experimental validation of the natural frequencies of the first four vibration modes in the directions in which motion is constrained

\subsection{Discussion}

Although the presented optimization procedure results in a major increase in stiffness in supporting directions, it also increases complexity of the obtained flexure designs. Using traditional manufacturing methods, the assembly of the proposed flexure designs can become complex. However, it is anticipated that rising additive manufacturing techniques will enable an effective and easy manufacturing of complex large-stroke flexure joints. As illustrated in section 2.4.2 SLS in nylon already allows for easy manufacturing without the need of assembly. Besides laser sintering in nylon, current technological developments show a strong advancement in direct metal printing like selective laser melting of titanium with wall thickness as small as $0.3 \mathrm{~mm}$.

\subsection{Conclusion}

In this paper, a method is presented for a systematic spatial topology optimization for flexure hinges including large deflection angles. This method includes a new adaption to a Nelder-Mead based optimization algorithm combined with a 3D building block-based layout synthesis method in order to optimize topology 
efficiently. The proposed method is used to design and experimentally validate a revolute joint with a 90 degrees range of motion. The optimized design provides an increase of about a factor ten in natural frequency of the first unwanted vibration mode with respect to the customary $\mathrm{TFCH}$, which represents a huge improvement in performance. Measurements on the natural frequencies of the first four vibrational modes are in good agreement with the modeled results and confirm the applicability of the proposed method. 


\subsection{Appendices}

\subsubsection{Optimization of a large stroke flexure hinge for off-axis support stiffness}

This appendix provides an additional optimization case for the optimization strategy presented in section 2.4 .

For this optimization, a stroke of -45 to 45 degrees deflection is considered combined with a maximum width of $85 \mathrm{~mm}$. Furthermore, selected material is tooling steel where we limit the allowable stress due to deformation to $600 \mathrm{MPa}$, about $1 / 3^{\text {rd }}$ of the yield stress. The considered optimization case aims at optimizing the support stiffness orthogonal to the axis of rotation (for this case the vertical support stiffness). The support stiffness for the shape-optimized initial reference layout (the TFCH consisting of leafsprings for the inner and outer building block) decreases to $250 \mathrm{~N} / \mathrm{mm}$ at the maximum deflection angle, which is less than $1 \%$ of the stiffness without deflection. The final optimized layout after two layout updates, consisting of a double TFCH for both the inner and outer building block, shows a minimum support stiffness of $2100 \mathrm{~N} / \mathrm{mm}$ over the range of motion, which is an increase in performance of about a factor eight. A CAD rendering of a prototype resulting from the optimized topology (made of duraform PA) is shown in Fig. 2.13 An overview of the support stiffness of intermediate shape optimizations is given in Table 2.6 
Table 2.6: Performance of the optimized shape for each intermediate layout for each iteration in layout

\begin{tabular}{lllll} 
Iteration & Outer flexures & Inner flexure & Stiffness $\left(45^{\circ}\right.$ deflection $)$ & Stiffness $\left(0^{\circ}\right.$ deflection $)$ \\
\hline \hline 1 & Leafspring & Leafspring & $2.5 \cdot 10^{2} \mathrm{~N} / \mathrm{mm}$ & $8.8 \cdot 10^{4} \mathrm{~N} / \mathrm{mm}$ \\
2 & Leafspring & Double TFCH & $8.0 \cdot 10^{2} \mathrm{~N} / \mathrm{mm}$ & $4.1 \cdot 10^{4} \mathrm{~N} / \mathrm{mm}$ \\
3 & Double TFCH & Double TFCH & $2.1 \cdot 10^{3} \mathrm{~N} / \mathrm{mm}$ & $6.3 \cdot 10^{4} \mathrm{~N} / \mathrm{mm}$ \\
\hline
\end{tabular}

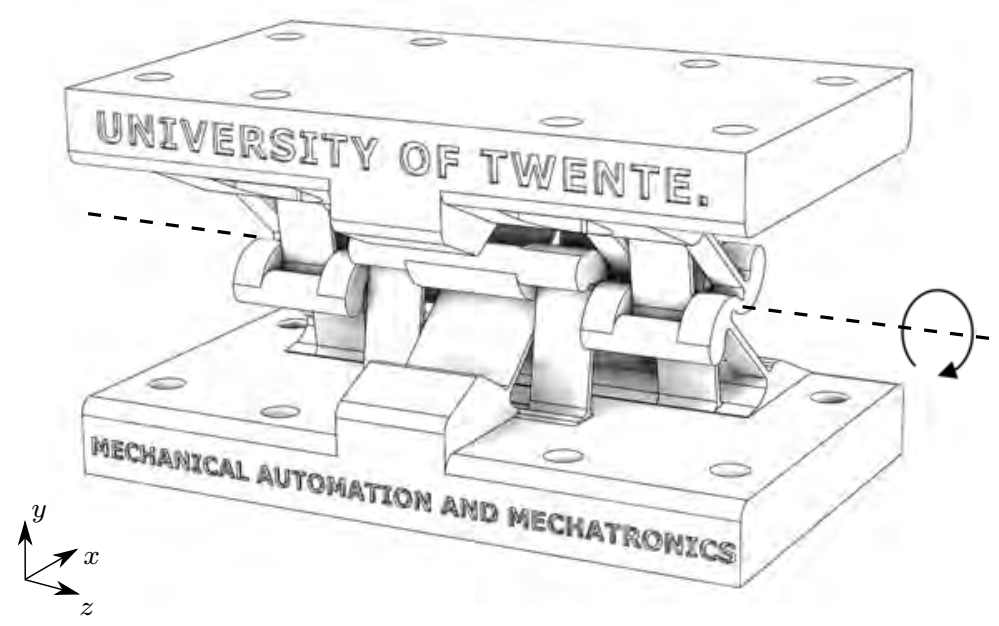

Figure 2.13: $\mathrm{CAD}$ rendering of the optimized flexure hinge with the dashed line indicating the rotation axis

\subsubsection{Intermediate optimization results}

This appendix provides the optimized shape for each intermediate layout of the results presented in section 2.4 The inner and outer building blocks are represented in red and blue respectively. The natural frequency of the first unwanted vibration mode and the maximum stress is given in the top left corner. The optimal parameter set $(\boldsymbol{p})$ is given in the bottom left corner. Note that the rigid body of the load, end effector and the base are not shown. 


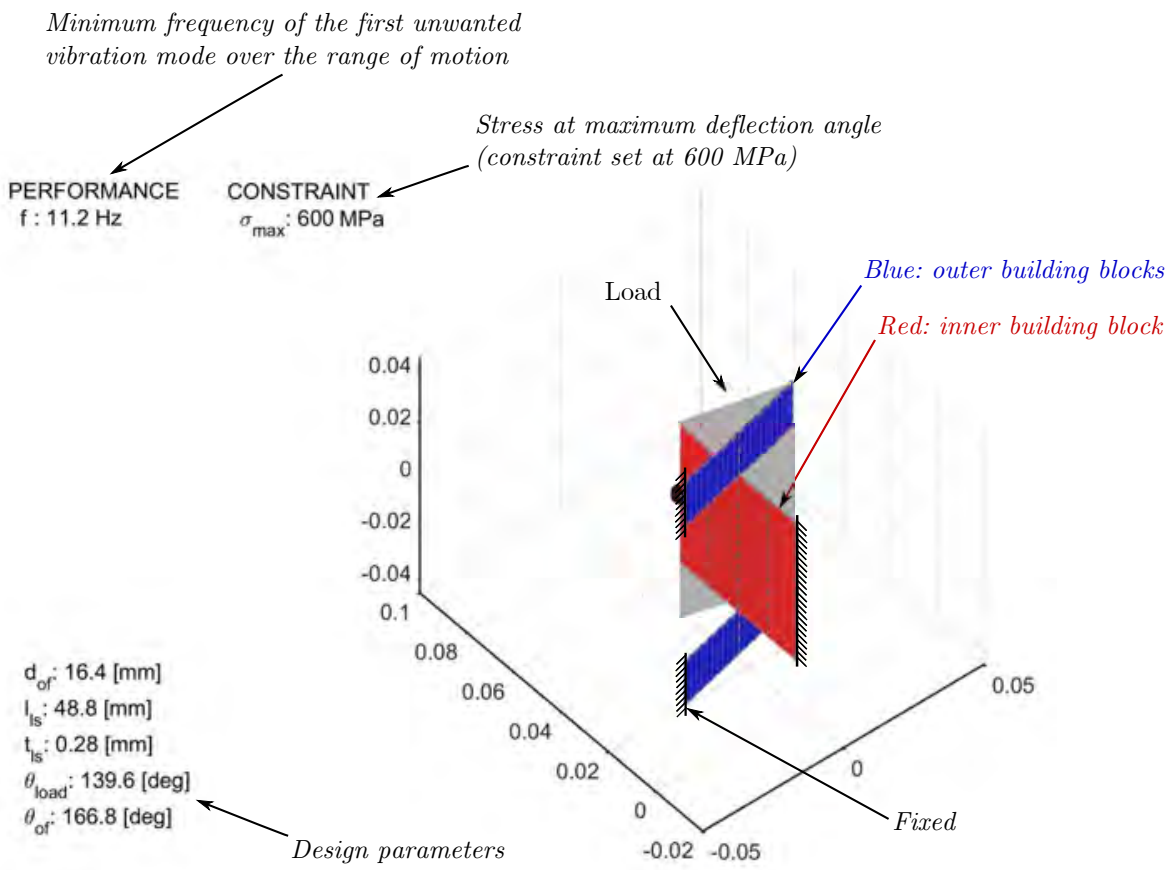

Figure 2.14: Optimized shape of the first layout including description
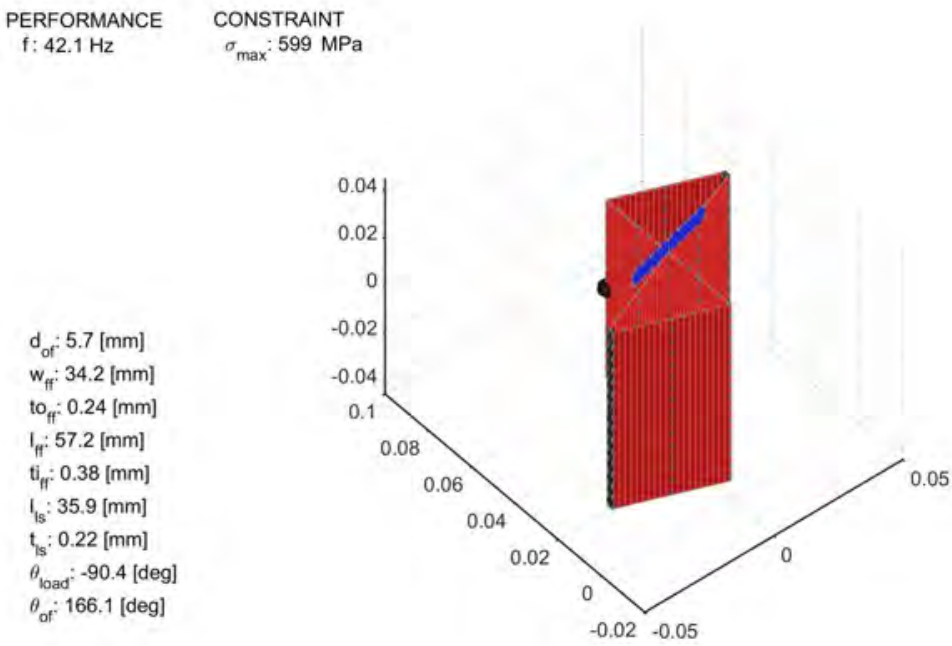

Figure 2.15: Optimized shape of the second layout 


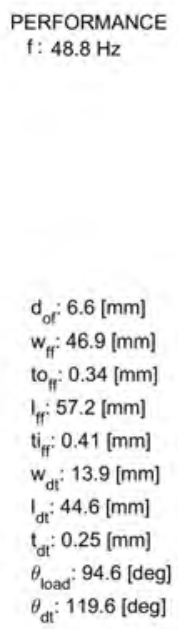

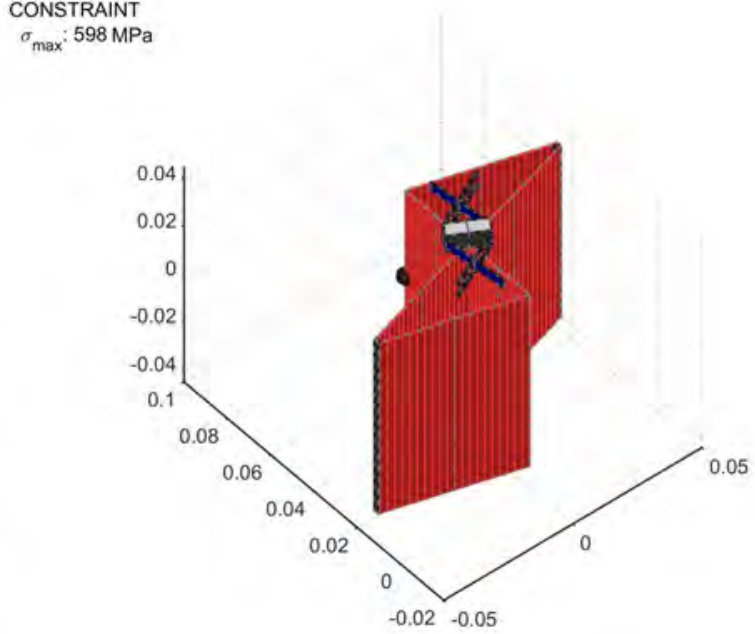

Figure 2.16: Optimized shape of the third layout

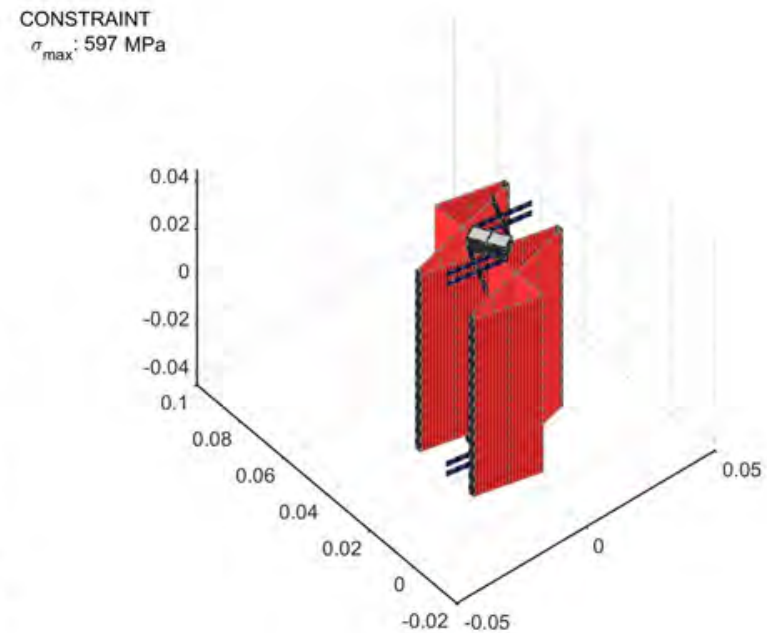

Figure 2.17: Optimized shape of the fourth layout 

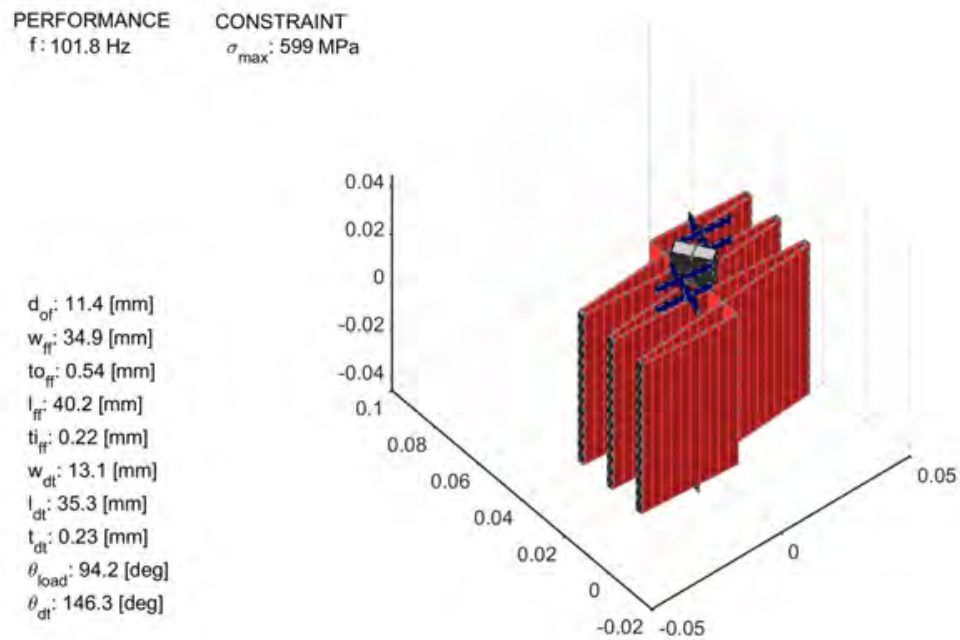

Figure 2.18: Optimized shape of the fifth and final layout 


\title{
Efficient collision detection method for flexure mechanisms comprising deflected leafsprings
}

\begin{abstract}
When designing and optimizing spatial flexure mechanisms, it is hard to predict collision due to $3 D$ motion and large deformations, which compromises the utilization of spatial freedom. A computationally efficient collision test is desirable to assure that feasible mechanism designs are found when algorithmically optimizing the shape of elastic mechanisms which are prone to collision. In this paper, a method is presented to test for collision specifically suited for flexure mechanisms by taking advantage of the typical slender aspect ratio and shape of the elastic members. Hereby, an efficient collision test is obtained that allows for the computation of a quantitative value for the severeness of collision. This value can then be used to efficiently converge to collision free solutions without excluding good mechanism designs leading to improved mechanisms, which utilize the maximum spatial design freedom.
\end{abstract}

\subsection{Introduction}

In precision applications often flexure-based mechanisms are used which allow motion by means of elastic deformation of slender elements. These mechanisms typically provide a small range of motion in which deterministic and repeatable

Published in: Journal of Mechanisms and Robotics (M. Naves, R.G.K.M. Aarts, D.M. Brouwer) 
motion is obtained allowing high precision manipulation. As long as small deformations are considered, collision of flexible members is often disregarded as their motion is only limited and relatively easy to predict. However, when the range of motion of flexure mechanisms is extended, collision becomes a complex matter especially in view of 3D motion. In order to optimize for the design of large stroke flexure mechanisms, a numerically efficient collision test suited for flexible mechanisms is required.

When optimizing the design of large stroke flexure hinges [27, 33, 69], collision of flexures is often neglected or inherently prevented by optimizing topologies which are fundamentally unable to collide. Preventing collision for relatively simple topologies which are prone to collision can be prevented by properly configuring the boundary conditions on the allowed designs. However, preventing collision for more complex large range of motion flexure joints [15, 108] or threedimensional spatial topologies, such as universal [65] 98] or spherical flexure joints [62 66], can become rather cumbersome and conservatively set constraints could exclude good mechanism designs during the optimization procedure.

For typical shape optimizations, the shape of a mechanism is defined by a set of design variables $(\boldsymbol{p})$ where the optimization objective is to find the optimal set of parameters which maximizes performance [28]. Often this problem is defined as a minimization problem of the objective function $(\mathcal{F})$ while satisfying one or more constraint functions $(\mathcal{C})$, for example

$$
\boldsymbol{p}_{\text {opt }}=\arg \min _{\boldsymbol{p}} \mathcal{F}(\boldsymbol{p}), \quad \text { subject to: } \quad \mathcal{C}(\boldsymbol{p}) \leq 0
$$

To avoid collision, we can consider the constraint function as a collision test which provides a value larger than zero when collision is present. Ideally, this constraint function is a continuous function in order to allow for proper convergence to collision free designs. If the constraint function is strongly discontinuous, the risk of getting stuck in local minima increases and convergence to the optimal solution is reduced, increasing computational cost. In order to obtain a continuous constraint function, a collision test is required that offer some kind of "penetration measure" like the penetration depth. This quantity specifies, e.g., a minimum translational vector with which the collided objects can be separated [107].

Many collision tests consider object bounding volumes which encapsulate the objects complex geometry by simple bounding volumes [14, 41, 96, 107]. Although this method has low computational cost due to its simple geometry, no accurate measure for the penetration depth and collision state can be obtained. 
In order improve accuracy, bounding volume-based collision tests are often supplemented by a more thorough collision test. A widely used method is to approximate the geometry by polyhedrons and to test whether representative points of one polyhedron are inside the other polyhedron [63]. By evaluating the distance with respect to the boundary of the considered polyhedron, the penetration depth can be evaluated. Drawback of this method is that actually obtaining the penetration depth is a difficult problem to solve and often requires an iterative solver, resulting in limited robustness and high computational cost [101].

An alternative method is to describe both objects to be tested by a collection of triangles and test for penetration of each vertex point through the planes of any triangle not including that vertex [26, 111]. Although this method does allow for a more accurate approximation of complex geometry, it still provides no definitive answer to the collision state. In order to verify the collision state, this method is often supplemented by a triangle-triangle-based intersection test which evaluates the intersecting points of the three edges of one triangle with respect to the plane containing the other triangle [61]. Hereby, the collision state and penetration depth can be evaluated. Although this method does not require an iterative solver, computational cost is significant and often many triangles are required to accurately approximate the objects geometry.

In this paper, a two-step approach for efficiently evaluating the penetration depth for flexure mechanisms without the need of a costly iterative solver is described. In a first filtering step, or "broad phase," a bounding-box based collision test is implemented for an efficient initial evaluation of the collision state. This initial test is complemented with a secondary geometrical intersection test, or "narrow phase," to accurately evaluate the penetration depth that utilizes the typical aspect ratio and shape of leafsprings to simplify the problem and to enhance computational efficiency. Due to this simplification, collision can be effectively included in structural optimizations for spatial flexible mechanisms to ensure collision-free designs.

\subsection{Collision test}

In this section, a collision test is described specifically suited for leafspring-based compliant mechanisms. First of all, a geometric reduction is outlined to simplify the collision test. Second, an initial collision test is discussed which is the broad phase to rule out collision effectively for objects which are unable to collide due to their diverse position in space. For the possible collisions remaining after the broad phase, a second geometric intersection test is employed in the narrow 
phase which includes an evaluation of the effective penetration depth to be used for a continuous constraint function in the optimization procedure. To ensure high efficiency, all computations are formulated such to allow for vectorized operations to prevent extensive for loops when evaluating for collision. In the described test, we will ignore the rigid parts and focus on collision between the deflected leafsprings of compliant mechanisms, which provide the main challenge in the collision test.

\subsubsection{Geometrical shape reduction}

In order to test for collision effectively, the geometric shape of a (deflected) leafspring is simplified. For the shape of the leafsprings, we assume leafsprings with a constant cross section and a relatively small thickness. Hence, to reduce complexity, the thickness of the leafspring is neglected to obtain a zero-thickness surface. Furthermore, when a leafspring is loaded by external forces, it will mainly deform in the compliant directions of the flexure corresponding to its degrees-of-freedom. For a leafspring, these deformations are given by the outof-plane bending and torsional deformations [72]. In order to incorporate these deformations, the shape of a leafspring is approximated with a discrete number of flat rectangular surfaces distributed along the length of the leafspring.

In case the thickness of the flexure does play an essential role for the collision state, the flexure thickness can be included by approximating the geometry by two coplanar flat surfaces. Furthermore, by choosing the perpendicular distance between both surfaces slightly larger than the actual thickness of the flexure, an additional separation distance can be included to ensure sufficient distance between the flexures over the range of motion. However, as including the thickness of the flexure does not change the described method in the remainder of this paper, flexure thickness is ignored in order to clarify schematic examples

An example of the reduction of two deflected leafsprings, each reduced to three surfaces, is given in Fig. 3.1 In the collision test, these straight surfaces will be used, assuming they represent the geometry of the deflected leafsprings and approximate their actual collision state. Surfaces $\mathbb{A}$ and $\mathbb{B}$ will be used in the successive sections for illustrative examples.

\subsubsection{Initial bounding volume test}

Directly testing for collision between all $n$ objects involves order $n^{2}$ geometry intersection tests which is computationally expensive. To reduce computational 


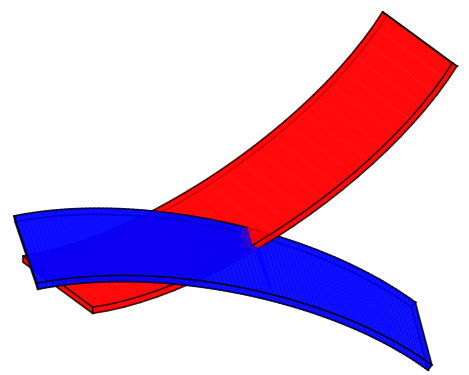

(a)

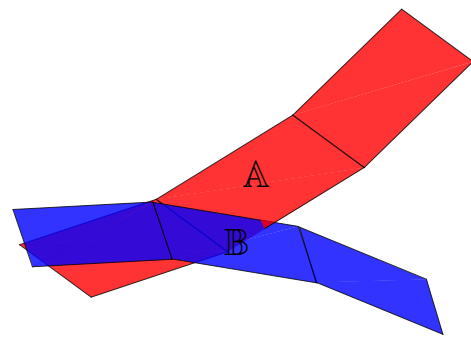

(b)

Figure 3.1: Geometrical reduction of two leafsprings: (a) original geometry and (b) simplified geometry consisting of six flat surfaces

cost, an approximate low cost initial collision test is used prior to the geometry intersection test to check if two objects are in their proximity and likely to collide. This allows for a faster collision test as one only needs to conduct a geometry intersection test for the parts of which the initial test indicates a possible collision.

An efficient class of approximate methods to test for collision are bounding volume collision tests [14 107]. Bounding volumes are simple volumes like boxes or spheres enclosing one or more objects with more complex shapes. The collision test for these bounding volumes are computationally advantageous compared to the tests for the objects they bound due to their simple geometry. The employed initial bounding volume test is based on an oriented bounding box test, which involves testing for overlap between two boxes which enclose both objects that have to be tested for collision [24]. As the considered objects are two flat surfaces, like the two surfaces $\mathbb{A}$ and $\mathbb{B}$ from Fig. $3.1 \mathrm{~b}$ aligning the bounding boxes with one of the surfaces results in a reduction of the volume of one of the bounding boxes to zero. This effectively leads to testing for overlap between a surface (the surface with which the bounding boxes are aligned) and the bounding box of the secondary surface equally aligned, which has an improved probability of ruling out collision. This oriented bounding box test for two arbitrary surfaces is schematically illustrated in Fig. 3.2 .

For the oriented bounding box test the surfaces $\mathbb{A}$ and $\mathbb{B}$ are considered, each spanned by the four vertices $\boldsymbol{A}_{0}, \ldots, \boldsymbol{A}_{3}$ and $\boldsymbol{B}_{0}, \ldots, \boldsymbol{B}_{3}$. Furthermore, we define 


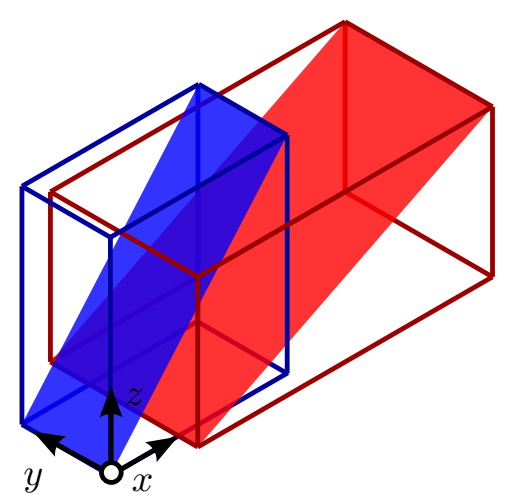

(a)

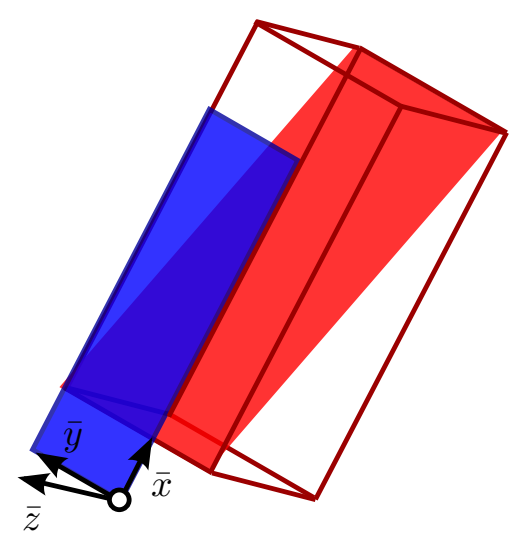

(b)

Figure 3.2: Schematic representation of bounding box alignment for two flat surfaces: (a) bounding boxes aligned with global axis $(x, y, z)$ and (b) oriented bounding boxes aligned with local axis $(\bar{x}, \bar{y}, \bar{z})$

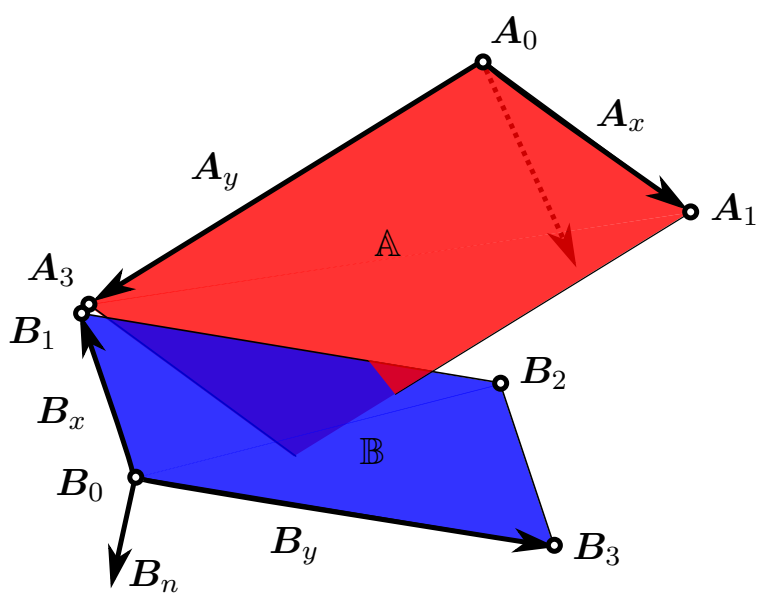

Figure 3.3: Schematic illustration of the directional vectors of surfaces $\mathbb{A}$ and $\mathbb{B}$. Note: vector $\boldsymbol{A}_{n}$ is hidden behind surface $\mathbb{A}$ 


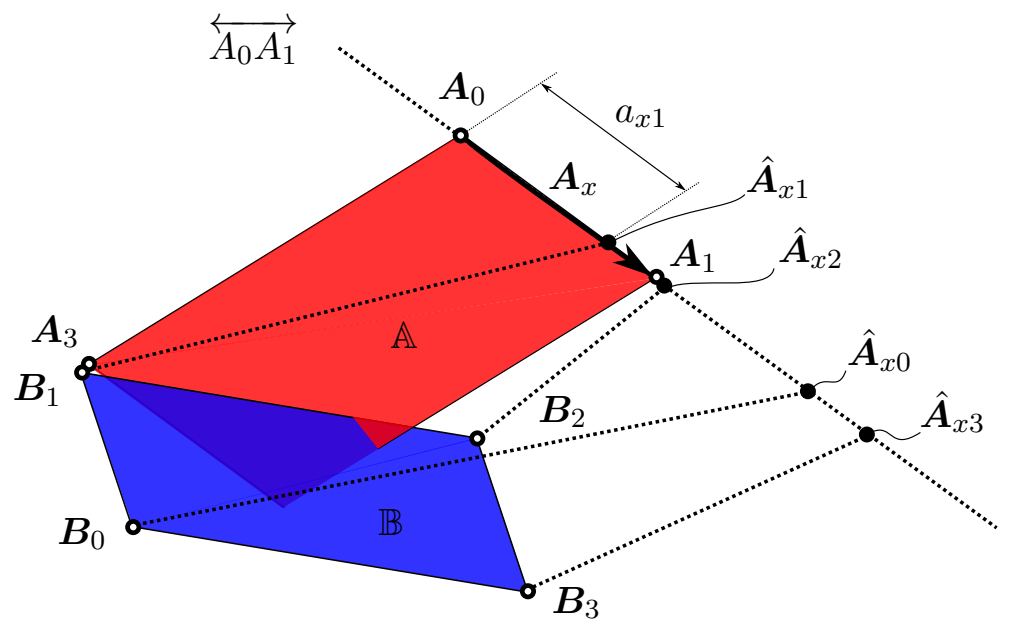

Figure 3.4: Scalar projection of vertices $\boldsymbol{B}_{0}, \boldsymbol{B}_{1}, \boldsymbol{B}_{2}$ and $\boldsymbol{B}_{3}$ on line $\overleftrightarrow{A_{0} A_{1}}$

the vectors $\boldsymbol{A}_{x}, \boldsymbol{A}_{y}, \boldsymbol{A}_{n}, \boldsymbol{B}_{x}, \boldsymbol{B}_{y}$ and $\boldsymbol{B}_{n}$ as

$$
\begin{array}{ll}
\boldsymbol{A}_{x}=\boldsymbol{A}_{1}-\boldsymbol{A}_{0} & \boldsymbol{B}_{x}=\boldsymbol{B}_{1}-\boldsymbol{B}_{0} \\
\boldsymbol{A}_{y}=\boldsymbol{A}_{3}-\boldsymbol{A}_{0} & \boldsymbol{B}_{y}=\boldsymbol{B}_{3}-\boldsymbol{B}_{0} \\
\boldsymbol{A}_{n}=\boldsymbol{A}_{x} \times \boldsymbol{A}_{y} & \boldsymbol{B}_{n}=\boldsymbol{B}_{x} \times \boldsymbol{B}_{y}
\end{array}
$$

These vectors are illustrated in Fig. 3.3 To test for overlap between surface $\mathbb{B}$ and the box enclosing surface $\mathbb{A}$ aligned with surface $\mathbb{B}$, all vertices of plane $\mathbb{B}$ are projected onto the three lines passing through $\boldsymbol{A}_{0}$ aligned with $\boldsymbol{A}_{x}, \boldsymbol{A}_{y}$ and $\boldsymbol{A}_{n}$, respectively. These scalar projections are given by

$$
\begin{array}{r}
\hat{\boldsymbol{A}}_{x i}=\boldsymbol{A}_{0}+a_{x i} \boldsymbol{A}_{x}, \quad \text { with: } a_{x i}=\frac{\boldsymbol{A}_{x} \cdot\left(\boldsymbol{B}_{i}-\boldsymbol{A}_{0}\right)}{\left\|\boldsymbol{A}_{x}\right\|^{2}} \\
\hat{\boldsymbol{A}}_{y i}=\boldsymbol{A}_{0}+a_{y i} \boldsymbol{A}_{y}, \quad \text { with: } a_{y i}=\frac{\boldsymbol{A}_{y} \cdot\left(\boldsymbol{B}_{i}-\boldsymbol{A}_{0}\right)}{\left\|\boldsymbol{A}_{y}\right\|^{2}} \\
\hat{\boldsymbol{A}}_{n i}=\boldsymbol{A}_{0}+a_{n i} \boldsymbol{A}_{n}, \quad \text { with: } a_{n i}=\frac{\boldsymbol{A}_{n} \cdot\left(\boldsymbol{B}_{i}-\boldsymbol{A}_{0}\right)}{\left\|\boldsymbol{A}_{n}\right\|^{2}} \\
\text { for: } i=0, \ldots, 3
\end{array}
$$

in which $a_{x i}, a_{y i}$ and $a_{n i}$ provide the distance between $\boldsymbol{A}_{0}$ and the projection of $\boldsymbol{B}_{i}$ scaled with the length of vector $\boldsymbol{A}_{x}, \boldsymbol{A}_{y}$ and $\boldsymbol{A}_{n}$, respectively. An example of these projections on the line passing through $\boldsymbol{A}_{0}$ aligned with $\boldsymbol{A}_{x}$, the line $\overleftrightarrow{A_{0} A_{1}}$, is schematically pictured in Fig. 3.4 In case all four projections on this line are not projected on line segment $\overline{A_{0} A_{1}}$ and are positioned on the same 
side of $\boldsymbol{A}_{0}$, collision cannot occur as the bounding boxes do not overlap. This also holds for the projection on line $\overleftrightarrow{A_{0} A_{3}}$, where collision is excluded when all projections are not on line segment $\overline{A_{0} A_{3}}$ and are located on the same side of $\boldsymbol{A}_{0}$. The projection on the line passing through $\boldsymbol{A}_{0}$ aligned with $\boldsymbol{A}_{n}$ excludes collision if all projections are on the same side of $\boldsymbol{A}_{0}$. Summarized, a collision is excluded when at least one of the following statements is true

$$
\begin{array}{lll}
a_{x i}<0 & \text { for } & i=0,1,2 \text { and } 3 \\
a_{x i}>1 & \text { for } & i=0,1,2 \text { and } 3 \\
a_{y i}<0 & \text { for } & i=0,1,2 \text { and } 3 \\
a_{y i}>1 & \text { for } & i=0,1,2 \text { and } 3 \\
a_{n i}<0 & \text { for } & i=0,1,2 \text { and } 3 \\
a_{n i}>0 & \text { for } & i=0,1,2 \text { and } 3
\end{array}
$$

If none of the six statements are satisfied, the bounding boxes overlap and a collision cannot be ruled out for the considered surfaces. Note that, for the considered surfaces $\mathbb{A}$ and $\mathbb{B}$ used in the example, none of the statements of Eq. (3.4) are satisfied, which is expected as the surfaces are actually colliding. In a similar way, an additional test can be conducted by testing for overlap between surface $\mathbb{A}$ and the box enclosing surface $\mathbb{B}$ aligned with surface $\mathbb{A}$, which can be conducted by projecting the vertices of plane $\mathbb{A}$ onto the three lines passing through $\boldsymbol{B}_{0}$ aligned with $\boldsymbol{B}_{x}, \boldsymbol{B}_{y}$ and $\boldsymbol{B}_{n}$. This will provides six additional statements which exclude a collision if at least one of these statements is true. If none of all twelve statements are satisfied, a further analysis is needed to verify the collision state as will be detailed next.

\subsubsection{Geometry intersection test}

In case collision cannot be excluded by the initial bounding volume test, a more thorough geometry intersection test is conducted. The goal of this test is to check for collision and, if colliding, evaluate the penetration depth of both surfaces to provide a continuous function for the "severeness of collision." We define the penetration depth for a collided surface pair as the total overlap distance, which is equal to the length of the line segment shared by both surfaces. It has to be noted that the length of this line segment is not necessarily equal to the length of the shortest translational vector with which the collided surfaces can be separated, a commonly used measure for the penetration depth. This modified definition is used as it allows for more efficient evaluation of the penetration depth while still providing a good continuous measure for the "severeness of collision." 


\section{Obtaining the line of intersection}

For the geometry intersection test, we initially consider two infinite reference planes co-planar to each surface. By definition, these reference planes will always intersect along a single line provided they are not exactly parallel. The parallel case will not be considered as this case will not give rise to a possible collision in the initial bounding volume test and hence will not occur in this second test. As the line of intersection is oriented perpendicular to the normal vectors of each surface, the direction of the line of intersection $(\boldsymbol{L})$ is given by [23]

$$
\boldsymbol{L}=\boldsymbol{A}_{n} \times \boldsymbol{B}_{n}
$$

Furthermore, the directional vector perpendicular to the line of intersection in plane $\mathbb{A}$ respectively plane $\mathbb{B}$ is given by

$$
\boldsymbol{A}_{p}=\boldsymbol{A}_{n} \times \boldsymbol{L}, \quad \boldsymbol{B}_{p}=\boldsymbol{B}_{n} \times \boldsymbol{L}
$$

Next we consider the two skew lines starting from $\boldsymbol{A}_{0}$ and $\boldsymbol{B}_{0}$ in the directions of $\boldsymbol{A}_{p}$, respectively $\boldsymbol{B}_{p}$, parameterized by

$$
\boldsymbol{l}_{A}(t)=\boldsymbol{A}_{0}+\boldsymbol{A}_{p} t, \quad \boldsymbol{l}_{B}(s)=\boldsymbol{B}_{0}+\boldsymbol{B}_{p} s
$$

Given that $\boldsymbol{A}_{p}$ and $\boldsymbol{B}_{p}$ are both perpendicular to the line of intersection (provided by Eq. (3.6)), the shortest distance between the skew lines $\boldsymbol{l}_{A}$ and $\boldsymbol{l}_{B}$ is exactly along the line of intersection. This holds as the shortest distance between two skew lines is equal to the length of the line segment perpendicular to both lines. Hereby, the location of the line of intersection can be found by determining the two points on line $\boldsymbol{l}_{A}$ and $\boldsymbol{l}_{B}$ with the shortest relative distance [86]. In order to find these locations we define the squared distance between both lines given by

$$
\begin{aligned}
& \boldsymbol{d}(t, s)= \\
& \left(\boldsymbol{l}_{A}(t)-\boldsymbol{l}_{B}(s)\right) \cdot\left(\boldsymbol{l}_{A}(t)-\boldsymbol{l}_{B}(s)\right)= \\
& \left(\boldsymbol{A}_{0}+\boldsymbol{A}_{p} t-\boldsymbol{B}_{0}-\boldsymbol{B}_{p} s\right) \cdot\left(\boldsymbol{A}_{0}+\boldsymbol{A}_{p} t-\boldsymbol{B}_{0}-\boldsymbol{B}_{p} s\right)
\end{aligned}
$$

At the minimum distance, the derivative of $d$ with respect to $t$ (or $s$ ) equals zero, resulting in

$$
\frac{\delta d(t, s)}{\delta t}=\left(2 \boldsymbol{A}_{p} t+2 \boldsymbol{A}_{0}-2 \boldsymbol{B}_{0}-2 \boldsymbol{B}_{p} s\right) \cdot \boldsymbol{A}_{p}=0
$$

Next by calculating the cross product with $\boldsymbol{B}_{p}$, variable $s$ can be eliminated

$$
\left(\left(\boldsymbol{A}_{p} \times \boldsymbol{B}_{p}\right) t+\boldsymbol{A}_{0} \times \boldsymbol{B}_{p}-\boldsymbol{B}_{0} \times \boldsymbol{B}_{p}\right) \cdot\left(\boldsymbol{A}_{p} \times \boldsymbol{B}_{p}\right)=0
$$


Then dividing by $\left\|\boldsymbol{A}_{p} \times \boldsymbol{B}_{p}\right\|^{2}$ and calculating the dot product with $\left(\boldsymbol{A}_{p} \times \boldsymbol{B}_{p}\right)$ yields

$$
t_{\text {min }}=\frac{\left(\left(\boldsymbol{B}_{0}-\boldsymbol{A}_{0}\right) \times \boldsymbol{B}_{p}\right) \cdot\left(\boldsymbol{A}_{p} \times \boldsymbol{B}_{p}\right)}{\left\|\boldsymbol{A}_{p} \times \boldsymbol{B}_{p}\right\|^{2}}
$$

This provides us with a point on the line of intersection, and therefore the location of the line of intersection, according to

$$
\boldsymbol{A}_{L}=\boldsymbol{A}_{0}+\boldsymbol{A}_{p} t_{\text {min }}
$$

which defines, in combination with the direction vector of Eq. 3.5, the location of the line of intersection. A schematic overview is provided at Fig. 3.5

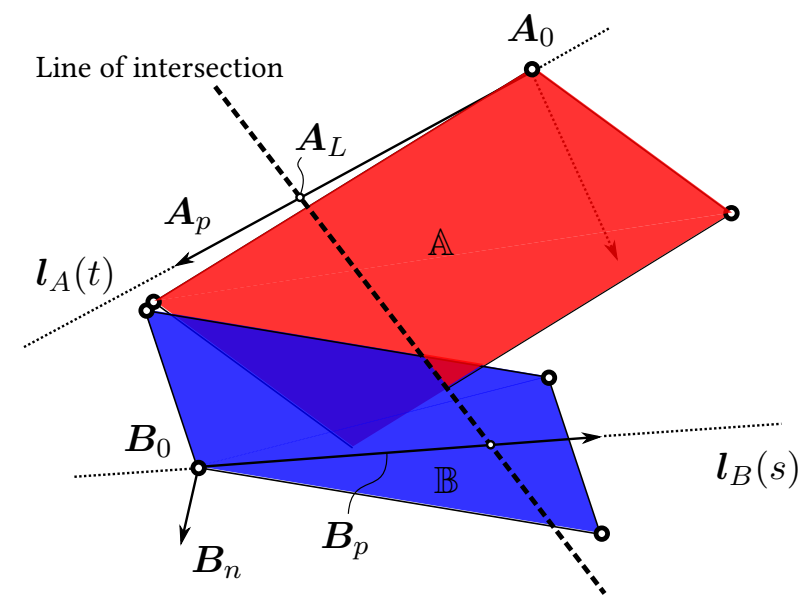

Figure 3.5: Line of intersection between surface $\mathbb{A}$ and $\mathbb{B}$ with $\boldsymbol{A}_{p}$ and $\boldsymbol{B}_{p}$ perpendicular to the line of intersection

\section{Evaluating the penetration depth}

To determine the collision state and to evaluate the penetration depth if the surfaces are colliding, the locations where the line of intersection crosses the outer ribs of each surface are evaluated.

First, the intersecting points between the line of intersection and lines $\overleftrightarrow{A_{0} A_{1}}$ and $\overleftrightarrow{A_{0} A_{3}}$ are evaluated, defined as $\boldsymbol{A}_{r x}$ and $\boldsymbol{A}_{r y}$. These points are found by the projection of $\boldsymbol{A}_{L}$ in a direction perpendicular to direction vector $\boldsymbol{A}_{p}$, or equivalently the vector $\boldsymbol{A}_{L}-\boldsymbol{A}_{0}$, on the lines through $\boldsymbol{A}_{0}$ in the directions $\boldsymbol{A}_{x}$ 


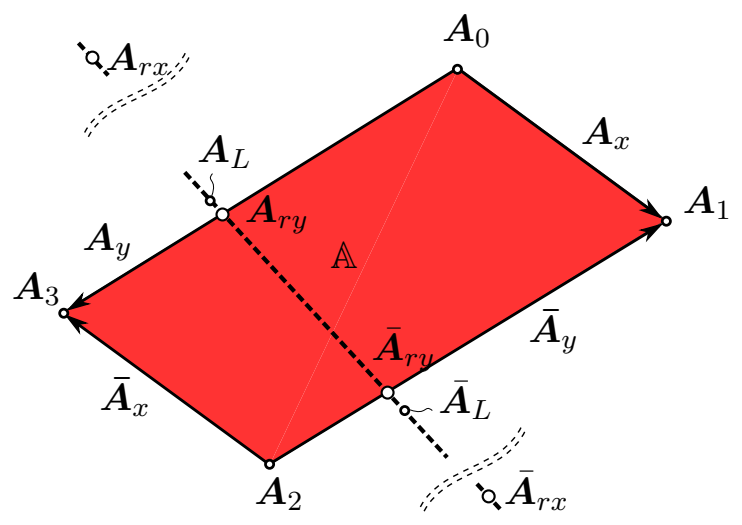

Figure 3.6: Intersecting points between the line of intersection and the outer ribs of surface $\mathbb{A}$

and $\boldsymbol{A}_{y}$, respectively. The results can be written as

$$
\begin{gathered}
\boldsymbol{A}_{r x}=\boldsymbol{A}_{0}+\boldsymbol{A}_{x} c_{x}, \quad \text { with: } c_{x}=\frac{\left\|\boldsymbol{A}_{L}-\boldsymbol{A}_{0}\right\|^{2}}{\left(\boldsymbol{A}_{L}-\boldsymbol{A}_{0}\right) \cdot \boldsymbol{A}_{x}} \\
\boldsymbol{A}_{r y}=\boldsymbol{A}_{0}+\boldsymbol{A}_{y} c_{y}, \quad \text { with: } c_{y}=\frac{\left.\| \boldsymbol{A}_{L}-\boldsymbol{A}_{0}\right) \|^{2}}{\left(\boldsymbol{A}_{L}-\boldsymbol{A}_{0}\right) \cdot \boldsymbol{A}_{y}}
\end{gathered}
$$

Next, the intersecting points of the line of intersection with the lines $\overleftrightarrow{A_{1} A_{2}}$ and $\overleftrightarrow{A_{2} A_{3}}$ are evaluated. For this purpose, the projection of $\boldsymbol{A}_{2}$ on the line of intersection is used, which is given by

$$
\overline{\boldsymbol{A}}_{L}=\boldsymbol{A}_{L}+\frac{\left(\boldsymbol{A}_{2}-\boldsymbol{A}_{L}\right) \cdot \boldsymbol{L}}{\|\boldsymbol{L}\|^{2}} \boldsymbol{L}
$$

The corresponding points of intersection are then given by

$$
\begin{array}{ll}
\overline{\boldsymbol{A}}_{r x}=\boldsymbol{A}_{2}-\boldsymbol{A}_{x} \bar{c}_{x}, \text { with: } & \bar{c}_{x}=\frac{\left\|\overline{\boldsymbol{A}}_{L}-\boldsymbol{A}_{2}\right\|^{2}}{\left(\overline{\boldsymbol{A}}_{L}-\boldsymbol{A}_{2}\right) \cdot\left(-\boldsymbol{A}_{x}\right)} \\
\overline{\boldsymbol{A}}_{r y}=\boldsymbol{A}_{2}-\boldsymbol{A}_{y} \bar{c}_{y}, \text { with: } & \bar{c}_{y}=\frac{\left\|\overline{\boldsymbol{A}}_{L}-\boldsymbol{A}_{2}\right\|^{2}}{\left(\overline{\boldsymbol{A}}_{L}-\boldsymbol{A}_{2}\right) \cdot\left(-\boldsymbol{A}_{y}\right)}
\end{array}
$$

A schematic overview is provided at Fig. 3.6. In case the line of intersection is not crossing the surface, no points of intersection are located on one of the outer ribs of the surface and collision is ruled out. This is the case when all values of $c_{x}$, $\bar{c}_{x}, c_{y}$ and $\bar{c}_{y}$ are outside the interval $0-1$. In case two values are in the interval 
$0-1$, the line of intersection crosses the surface and the surface pair potentially collides.

To evaluate the intersecting points with respect to surface $\mathbb{B}$, a similar procedure can be used. When for surface $\mathbb{B}$ also two intersecting points exists, the collision state and penetration depth can be evaluated. For this, we evaluate the relative position between the intersecting points along the line of intersection, schematically shown in Fig. 3.7 where $\boldsymbol{A}_{r 1}, \boldsymbol{A}_{r 2}, \boldsymbol{B}_{r 1}$ and $\boldsymbol{B}_{r 2}$ represent the intersecting points with the outer ribs of the surfaces. Note that for the considered case in Fig. 3.6 we can write $\boldsymbol{A}_{r 1}=\boldsymbol{A}_{r y}$ and $\boldsymbol{A}_{r 2}=\overline{\boldsymbol{A}}_{r y}$. Next, the distance of the intersecting points with respect to reference point $\boldsymbol{A}_{L}$ is determined which provide their relative position along the line of intersection

$$
\begin{array}{lll}
a_{r i}=\left\|\boldsymbol{A}_{r i}-\boldsymbol{A}_{L}\right\|, & \text { for: } & i=1,2 \\
b_{r i}=\left\|\boldsymbol{B}_{r i}-\boldsymbol{A}_{L}\right\|, & \text { for: } & i=1,2
\end{array}
$$

Finally, the penetration depth $\lambda$ is provided by the overlapping length of the two line segments connecting $\boldsymbol{A}_{r 1}$ and $\boldsymbol{A}_{r 2}$, respectively $\boldsymbol{B}_{r 1}$ and $\boldsymbol{B}_{r 2}$ provided by

$$
\begin{aligned}
\lambda & =\min \left(\max \left(a_{r 1}, a_{r 2}\right), \max \left(b_{r 1}, b_{r 2}\right)\right) \\
& -\max \left(\min \left(a_{r 1}, a_{r 2}\right), \min \left(b_{r 1}, b_{r 2}\right)\right)
\end{aligned}
$$

For the case $\lambda$ is negative, the surfaces do not overlap and $\lambda$ provides the separation distance between both surfaces measured along the line of intersection of the infinite reference planes. For the case $\lambda$ is positive, collision does occur and the value of $\lambda$ gives the total penetration depth, which has been defined as the length of the line segment shared by the collided surface pair. In order to provide a value for the "severeness of collision" for the entire mechanism, the penetration depth of each tested surface pair is summed $\left(\lambda_{\text {sum }}\right)$ over the range of motion to provide a measure for the combined penetration depth. This value can then be used for the constraint function in an optimization procedure to guide the procedure toward mechanism designs which are free of collision.

Although $\lambda_{\text {sum }}$ provides a continuous function with respect to the shape of the tested geometry, it has to be noted that the derivative of $\lambda_{\text {sum }}$ is not necessarily continuous due to the sharp rise of penetration depth at the instance intersection starts. Therefore, this method could be unsuited for optimization procedures which require the availability of a continuous derivative of the constraint function. 


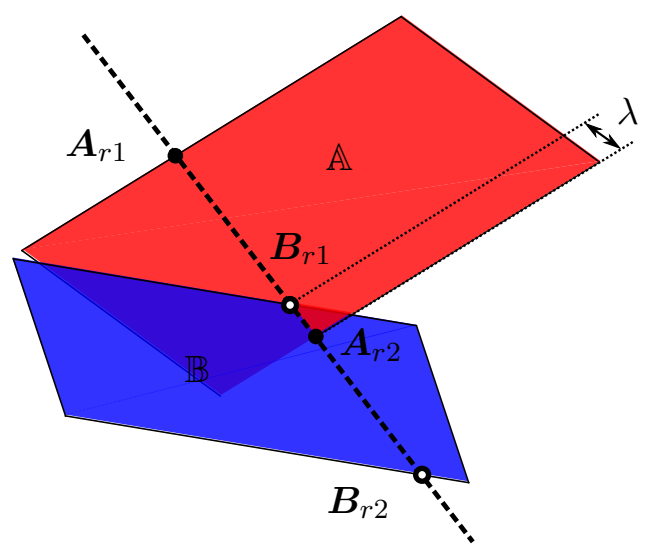

Figure 3.7: Relative position of the intersecting points with line of intersection

\subsection{Verification}

To verify the described collision test, the summed penetration depth $\lambda_{\text {sum }}$ of a flexure-based torsion spring as a function of two design parameters is evaluated. The considered torsion spring consists of three co-radial positioned curved leafsprings with a constant pitch (Fig. 3.8 ) and a radius of $50 \mathrm{~mm}$. The torsion spring has a range of motion of \pm 90 degrees rotation around the $z$-axis and each leafspring has a cross-sectional dimension of $10 \times 2 \mathrm{~mm}$. Selected material is nylon with a young's modulus of $1.5 \mathrm{GPa}$ and a shear modulus of $0.5 \mathrm{GPa}$. Furthermore, the total angle of the revolutions of the curved leafsprings ( $n$ in degrees, with 360 degrees being a single revolution) and the total height ( $h$ in $\mathrm{mm}$ ) are the evaluated design parameters.

In order to compute the deflected state of the torsion spring, the flexible multibody program SPACAR is used [45]. This program uses a series of interconnected non-linear finite beam elements where flexibility of the elements is naturally included due to the discrete deformation modes of the elements. Furthermore, it allows for the simulation of initially precurved stress-free beams [58]. The deformed state of a flexible torsion spring at -90 and +90 degrees deflection for $n=360$ degrees and $h=50 \mathrm{~mm}$ is given at Fig. 3.9

\subsubsection{Collision test continuity}

In order to evaluate the continuity of the collision test, the penetration depth $\lambda_{\text {sum }}$ is computed for a range of values for the design parameters $n$ and $h$. For the 


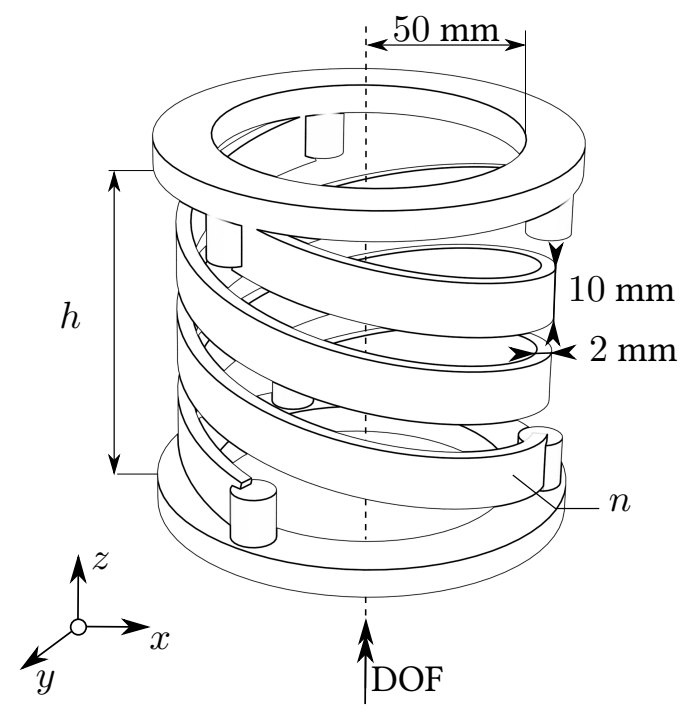

Figure 3.8: Parameterized model of the cylindrical torsion spring with height $h$ (in $\mathrm{mm}$ ) and total rotation angle of the undeformed curved leafsprings $n$ (in degrees). The width $b$ and thickness $t$ of the leafsprings are fixed to $10 \mathrm{~mm}$ and $2 \mathrm{~mm}$ respectively.

collision test, each leafspring is modeled with 12 precurved beam-elements and the shape of each element is approximated with three flat surfaces. Furthermore, the collision state is evaluated at 50 steps over the range of motion over which the values of $\lambda$ are summed. A visualization of the penetration depth $\lambda_{\text {sum }}$ for $20<h<100 \mathrm{~mm}$ and $360<n<1080$ degrees is given in Fig. 3.10 .

From this figure it can be observed that the summed penetration distance provides a continuous function with respect to the selected design parameters. Due to this continuity, it is well suited for a constraint function in optimization procedures and it allows for proper convergence to collision free designs.

\subsubsection{Computational efficiency}

To give an indication of the efficiency of the described collision test, the required computational time for testing for collision for $h=20 \mathrm{~mm}$ and $n=1080$ degrees is evaluated. For this case, a large number of surfaces are colliding (see Fig. 3.10). For the collision test, the model consists of 36 beam-elements each modeled with 3 flat surfaces (total of 108 unique surfaces). Furthermore, collision is tested at 50 steps over the range of motion, resulting in a total of $(108 \times 107 \times 50) / 2=$ 


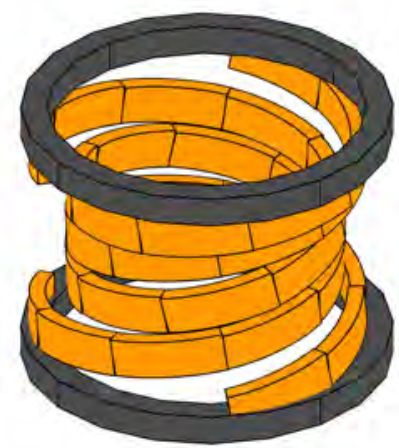

(a)

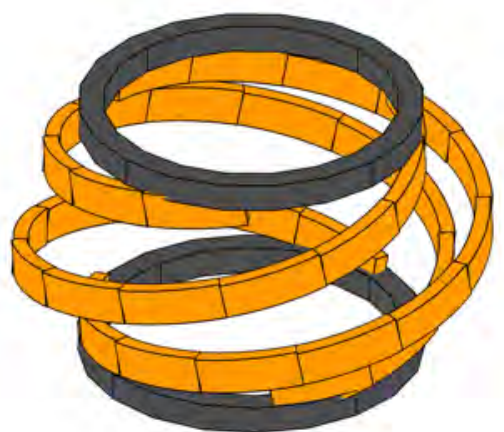

(b)

Figure 3.9: Deflected state of a torsion spring with each leafspring modeled with 12 flexible beam elements. The deflected state is given for design parameter settings $n=$ 360 degrees and $h=50 \mathrm{~mm}$ which just avoids collision at (a) -90 and (b) +90 degrees deflection

288900 individual tests. For this specific case, evaluating the total penetration depth is conducted within 0.5 seconds.

In order to provide a reference for the computational cost, this intersection test is compared to a triangle-triangle-based intersection test described by Möller 61] at which the geometry is re-approximated with 216 triangles where each original rectangular surface is split into two triangles. This equivalent collision test takes approximately 47 seconds for the evaluation of 1161000 surface pairs. It has to be noted that the accuracy and computational cost of this reference test has been increased due to the increased number of surfaces. However, by dividing the total computational cost with the total number of collision tests, the time required for a single test can be evaluated. For the collision test described in this paper, the required time for a single test is approximately $2 \mathrm{~ns}$, compared to $41 \mathrm{~ns}$ for Möller's algorithm, which is about 20 times faster

An overview of the collision test statistics is given in Table 3.1 It has to be noted that the actual computational time required for the collision test is dependent on the implementation of the algorithm. However, it still provides a good indication of the required computational cost for both methods. 


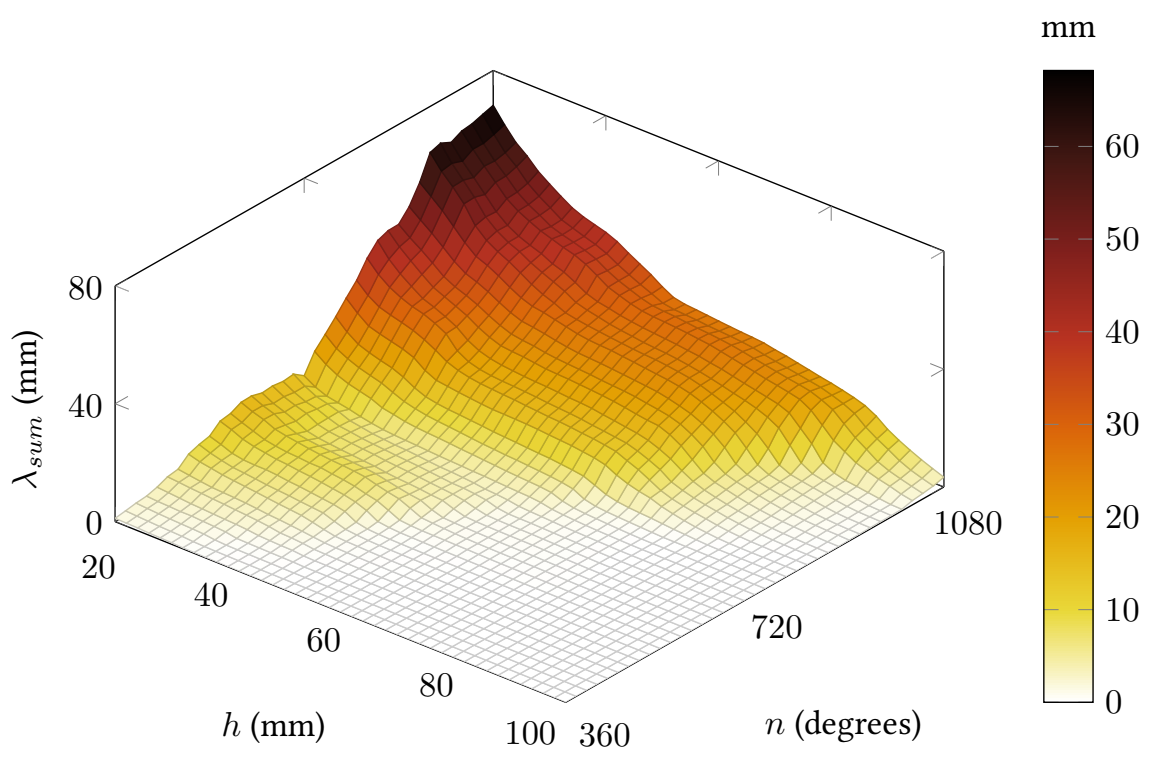

Figure 3.10: Summed penetration depth $\left(\lambda_{\text {sum }}\right)$ as function of design parameters $n$ and $h$

Table 3.1: Comparison of computational cost with respect to Möller's algoritm

\begin{tabular}{lll} 
& Presented algorithm & Möller's algorithm \\
\hline \hline Number of surfaces & 108 & 216 \\
Number of collision tests & 288900 & 1161000 \\
Total computational time & $0.5 \mathrm{~s}$ & $47.2 \mathrm{~s}$ \\
Time for single collision test & $2 \mathrm{~ns}$ & $41 \mathrm{~ns}$ \\
\hline
\end{tabular}

\subsection{Discussion}

For the geometric reduction of the flexures, the geometry of the leafsprings is reduced to a set of flat surfaces approximating a zero-thickness leafspring. For most cases, the thickness of the flexures is sufficiently small to be negligible. However, if it is required to include the thickness of the flexure or when sufficient "clearance" between the flexures is required to prevent collision when considering, e.g., manufacturing tolerances, a finite thickness of the surfaces can be implemented. This finite thickness can be added to the initial bounding box 
test by slightly increasing the bounding box size matching the flexure thickness. For the evaluation of the penetration depth, however, only zero thickness surfaces are supported by the described method. Therefore, in order to include the thickness of the flexures, two sets of co-planar surfaces can be used to resemble the correct thickness.

In order to include other objects in the collision test, such as intermediate rigid bodies of the mechanism or obstacles, additional sets of flat surfaces can be used. These surfaces can then be included in the collision test to avoid collision with the additional objects. Again, these objects have to be described with rectangular surfaces in order to take advantage of the algorithm described in this paper.

\subsection{Conclusion}

In this paper, a fast collision evaluation method for spatial flexure mechanism is presented. It efficiently evaluates the penetration depth between colliding flexures, which is crucial for enabling fast constrained optimizations. By including the penetration depth as a soft constraint on collision, the optimization procedure efficiently converges to just collision free designs.

The efficiency of the collision test is obtained by (1) using a two-step approach and (2) the assumption of thin flexures. The first fast step in the two-step approach uses a bounding-box-based collision test which provides an initial evaluation of the collision state. If this test yields possible collision, a second more accurate geometrical intersection test verifies the collision state and, if colliding, provides a measure for the penetration depth. The typically small thickness and deformation modes of the flexures permit subdividing of the flexures into a discrete number of flat surfaces, which can be evaluated for the penetration depth with projective geometry. Therefore, no iterative solver is required and the penetration depth can be computed fast.

With the presented collision detection method, optimization procedures are able to efficiently avoid collision while utilizing the maximum geometric design freedom, thereby improving spatial flexure mechanism designs. 



\title{
Chapter 4
}

\section{Large stroke high off-axis stiffness three degree of freedom spherical flexure joint}

\begin{abstract}
Three degree of freedom spherical flexure joints are typically limited to small deflections because of their strong loss of stiffness in support directions when deflected. In this paper, a smartly stacked folded leafspring based large stroke spherical flexure joint is presented which can maintain a high level of support stiffness over $a \pm 30$ degrees tip-tilt and $a \pm 10$ degrees pan motion. To enable this large range of motion, two sets of three leafsprings are stacked in series. A large loss of support stiffness over the range of motion due to the underconstrained intermediate body is avoided by assuring that the instant centers of rotation coincide. To mitigate internal dynamics caused by the underconstraints, a design with underconstraint eliminators and a design with eddy current damping are presented. Furthermore, the design of the spherical joint has been optimized which has resulted in a flexure joint design which can achieve a support stiffness of over $200 \mathrm{~N} / \mathrm{mm}$ and a load capacity of $290 \mathrm{~N}$ at the maximum deflection angle. Experimental measurements validate the high level of support stiffness and load capacity over the entire range of motion.
\end{abstract}

\subsection{Introduction}

In high precision applications flexure-based mechanisms are used for their deterministic behavior due to the absence of play and friction. Spherical flexure joints are often encountered in flexure-based precision spatial manipulators with

Published in: Precision Engineering (M. Naves, R.G.K.M. Aarts, D.M. Brouwer) 
parallel kinematic arrangements [13, 62, 82], such as spatial (6 DOF) nanapositioners [81, 110], microassembly [88] and precision alignment systems [7, 79]. For this purpose, typically spherical notch joints [15, 56] or short wire flexures [13, 47 79] are used which provide the required spherical motion. These joints provide a small and compact design and allow for easy manufacturing (Fig.4.19). However, they only provide a limited range of motion (typically within a few degrees) due to their localized compliance, causing high stress levels already at small deflection angles. Therefore, they are mostly used for optical alignment systems requiring only small rotation angles of the spherical joints.

In order to allow for larger rotation angles, spherical joints can be manufactured by a stacked arrangement of wire flexures [39, 40, 84, 92] (Fig. 4.13). Although this type of joint allows for a larger range of motion, it suffers from a low level of support stiffness in combination with a strong decrease of the support stiffness with increasing deflection angle. Furthermore, due to their long and slender geometry, they are prone to buckling and only provide a limited load capacity.

Alternatively, three single degree of freedom flexure joints can be concatenated in order to obtain the required degrees of freedom. A commonly used design consists of three cruciform hinges [90] placed in series with intersecting rotation axis [7] 98], or a stacked arrangement of single degree of freedom cross spring pivots [82, 97] (Fig. 4.1 k). Although concatenation of single degree of freedom flexure joints allows for larger rotation angles, support stiffness remains limited and a rather large and bulky design is obtained due to the serial stacking. Especially when considering a large range of motion when used in a parallel kinematic arrangements, it can be difficult to avoid interference of the parallel arrangement due to the size of the spherical joints. Furthermore, if the rotation axis of each individual joint does not intersect in a single point, parasitic motion of the joints will strongly affect its kinematic behavior and can result in difficulties with respect to control.

In this paper, a flexure-based spherical joint design is presented which uses folded leafsprings as flexible element to obtain the required degrees of freedom. A folded leafspring based design for a spherical joint has been presented before by Schellekens et al. [85] who combine three parallel folded leafsprings. This design allows for a high support stiffness and load capacity although only a limited range of motion is obtained. In order to extend the range of motion while maintaining high support stiffness, alternative spherical flexure joint concept topologies are presented. All concept topologies are optimized and compared in order to investigate the influence of certain design parameters and their relation with the required range of motion on its performance. A prototype is realized 
for an experimental validation of the simulated performance. Additionally, two options are presented to mitigate internal dynamics caused by underconstraints.

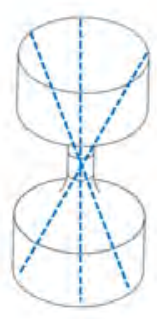

(a)

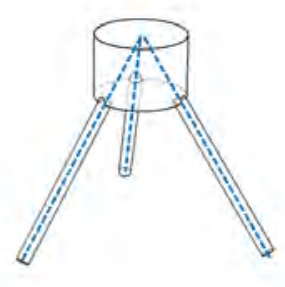

(b)

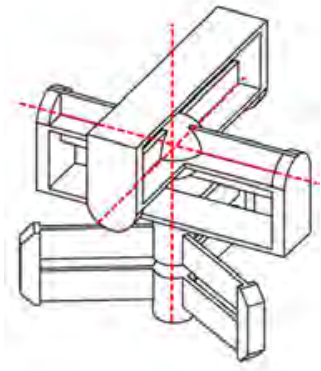

(c)

Figure 4.1: Flexure-based spherical joints: (a) spherical notch joint [15, 56, 110] or short wire flexure [13, 47, 79], (b) wire flexure-based spherical joint [39, 84, 92, 104], (c) spherical joint constructed by concatenating three single DOF joints [7 98$]$

\subsection{Notational convention for spherical motion}

Most flexure-based spherical joints, e.g. the spherical joints illustrated in Fig.4.1 are characterized by a rotational symmetric design. Due to this rotational symmetry, a distinct "pan" and "tip-tilt" motion can be identified, each with its own characteristic deformations of the flexible members and corresponding behavior of the joint.

Tip-tilt motion is defined by rotational motion around an arbitrary axis perpendicular to the symmetry axis. As each rotational axis perpendicular to the symmetry axis is allowed, tip-tilt motion governs two rotational degrees of freedom. For all spherical flexure joints in this paper, tip-tilt is provided by rotations with the axis of rotation positioned in the $x y$-plane, which is then perpendicular to the symmetry axis ( $z$-axis). To specify tip-tilt motion, we define the relative orientation of the tilt axis $\left(\boldsymbol{R}_{t}\right)$ with respect to the $x$-axis by angle $\phi$ given by

$$
\boldsymbol{R}_{t}=\left[\begin{array}{c}
\cos (\phi) \\
\sin (\phi) \\
0
\end{array}\right]
$$

with the magnitude of the rotation specified by $\omega_{t}$, see Fig. 4.2 Pan motion is defined by a rotational motion around the symmetry axis governing the remaining degree of freedom. To specify pan motion, the pan axis $\left(\boldsymbol{R}_{p}\right)$ is oriented 


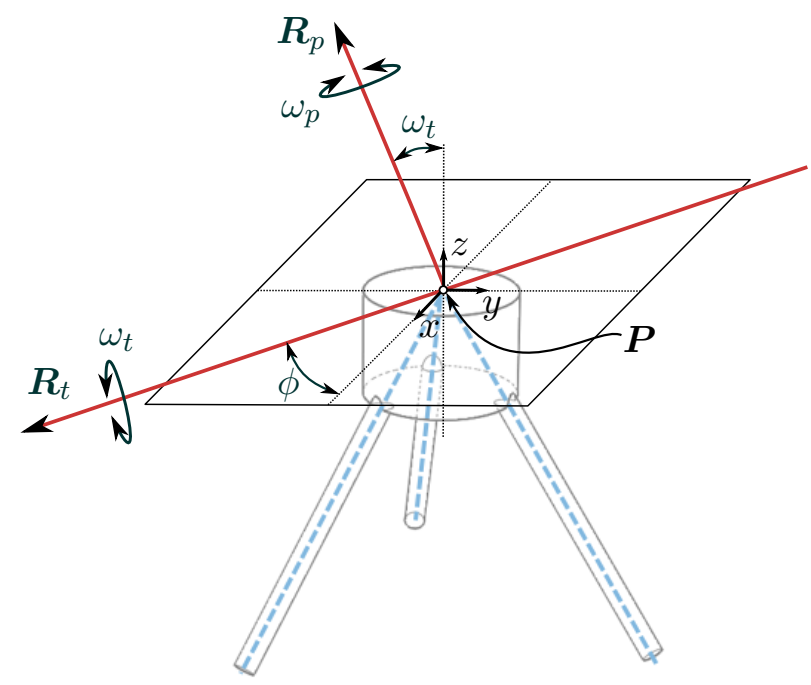

Figure 4.2: Schematic illustration of the tip-tilt and pan motion to express spherical joint motion, with the instant center of location defined by $\boldsymbol{P}$

perpendicular to the tilt axis co-rotational with the tilt motion, initially aligned with the symmetry axis, i.e.

$$
\boldsymbol{R}_{p}=\left[\begin{array}{c}
\sin (\phi) \sin \left(\omega_{t}\right) \\
-\cos (\phi) \sin \left(\omega_{t}\right) \\
\cos \left(\omega_{t}\right)
\end{array}\right]
$$

with the magnitude of pan motion denoted by $\omega_{p}$.

\subsection{Controlling degrees of freedom}

A spherical flexure joint is characterized by the property to allow motion in the three rotational degrees of freedom while motion in all translational directions are constrained (the directions in which load-bearing support is provided). For many applications, a spherical joint with coinciding rotation axes is required to concentrate all rotational motion in a single point, the instant center of rotation $(\boldsymbol{P})$. This freedom space, which contains a collection of all admissible axis of rotation, is schematically illustrated in Fig. 4.3] [37].

The complementary constraint space, which provides the required translational constraints for the given freedom space, is given by all translational constraints 


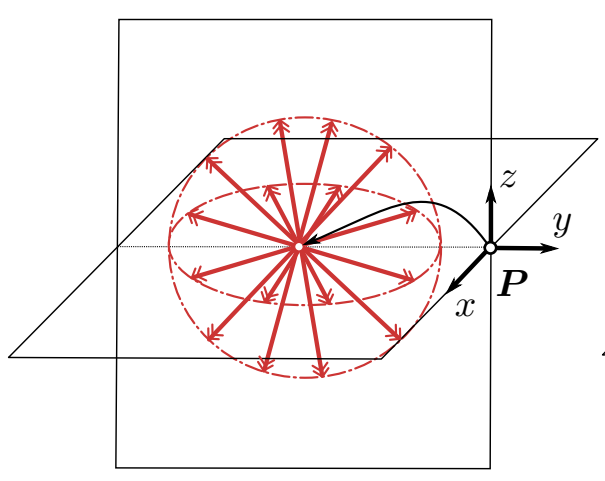

(a)

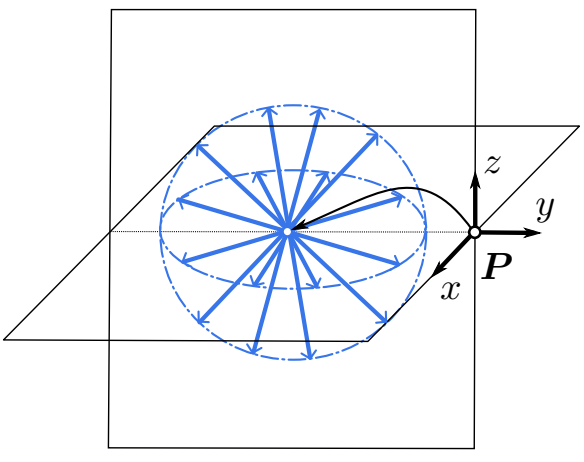

(b)

Figure 4.3: (a) Schematic illustration of the freedom space with all rotational degrees of freedom and (b) the complementary constraint space with all translational constraints for a spherical flexure joint with the instant center of rotation indicated by $\boldsymbol{P}$ [37]

intersecting the instant center of rotation, illustrated in Fig. $4.3 \mathrm{~b}$. This constraint space is also visible in the spherical joint designs provided in Fig. 4.1 In order to obtain this constraint space, flexible members are required which only constrain a single degree of freedom. Wire flexures are suited for this purpose, but do not allow for both a large range of motion and a high support stiffness [92]. Therefore, folded leafsprings are used which also constrain a single translational degree of freedom, which is located along the intersection line of the two leafsprings. Compared to wire flexures, the folded leafsprings typically allow for a larger range of motion with a higher level of support stiffness and load capacity.

\subsection{Spherical joint concept topologies}

In this section three different types of folded-leafspring based spherical joints are presented. The first type consists of a rotational symmetric set of parallel folded leafsprings. The second type uses two rotational symmetric sets stacked in parallel and the third type stacks the two sets in series.

\subsubsection{Folded Leafspring based spherical joint (FL joint)}

The most elementary topology for a folded-leafspring based spherical flexure joint consist of a set of folded leafsprings directly connected between the fixed 


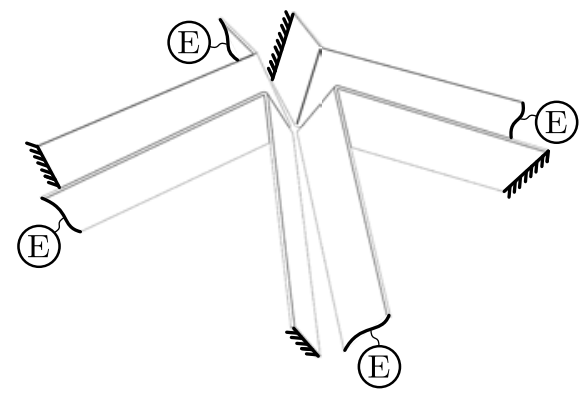

(a) $\mathrm{FL}-\mathrm{r}_{4} \mathrm{p}_{1}$

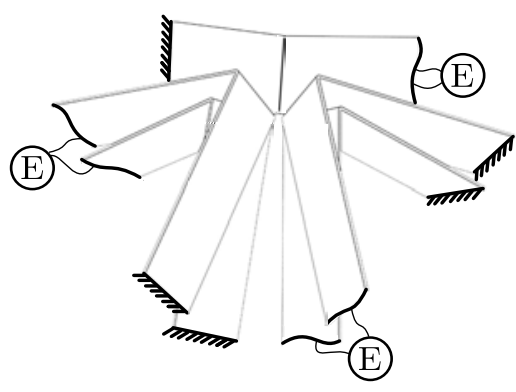

(b) FL- $\mathrm{r}_{3} \mathrm{p}_{2}$

Figure 4.4: Folded Leafspring based spherical joint topology (a) FL-r $\mathrm{r}_{4} \mathrm{p}_{1}$ : four rotational symmetric folded leafsprings (b) FL- $\mathrm{r}_{3} \mathrm{p}_{2}$ : two sets of three rotational symmetric folded leafsprings with a different orientation angle. "E" represents the connections with the end effector.

world and end effector. To comply with the constraint space as described in section 4.3 all folding lines require to intersect at a single point which than defines the instant center of rotation. An exact constrained design is obtained with three folded leafsprings.

In order to distinguish this topology from other topologies discussed hereafter, it is named the "Folded Leafspring based spherical joint", abbreviated to FL joint. When also considering overconstrained designs in order to potentiality improve support stiffness, more variations on this topology can be generated. First of all, the number of rotational symmetric folded leafsprings around the pan axis can be increased, illustrated in Fig. 4.4a. Secondly, multiple folded leafsprings can be positioned in parallel under a different orientation angle (given by the angle between the folding line of the leafspring and the symmetry axis). An example of a FL joint with two sets of folded leafsprings with slightly different orientation angle is provided in Fig. $4.4 \mathrm{~b}$ As the deformations of the two sets are similar, a small offset in orientation angle is typically sufficient in order to prevent collision between the parallel flexures. To identify the different joint topologies, $r_{n}$ is used to indicate $n$ number of rotational symmetric folded leafsprings and $\mathrm{p}_{n}$ is used to indicate $n$ sets of folded leafsprings positioned in parallel with different orientation angle. 


\subsubsection{Parallel stacked Folded Leafspring based spherical joint (PFL joint)}

A more elaborate joint topology is obtained when two FL joints are placed in parallel between the fixed world and end effector, the so-called "Parallel stacked Folded Leafspring based spherical joint", abbreviated to PFL joint. This topology combines two FL joints where one of them is a duplicate of the other mirrored over a plane perpendicular to the symmetry axis, positioned such to obtain coinciding instant center of rotations. Furthermore, in order to avoid collision between flexures, the mirrored stage is rotated with $180 / \mathrm{r}_{n}$ degrees around the symmetry axis. Effectively this leads to two FL joints placed in parallel between the fixed world and end effector, similar to the FL- $\mathrm{r}_{n} \mathrm{p}_{2}$ joint topology. However, due to the different orientation of the second set of folded leafsprings, different mechanical properties are obtained. For example, due to the more even distribution of the folded leafsprings over the circumference of the spherical joint, less variation of the mechanical properties with respect to the tip-tilt direction $\phi$ is obtained. A schematic illustration of the decomposition of the PFL joint is provided in Fig. 4.5. For this topology, multiple sets of folded leafsprings with a different orientation angle are not considered due to the limited build-space available.

\subsubsection{Serial stacked Folded Leafspring based spherical joint (SFL joint)}

The third type of flexure topology is geometrically identical to the PFL joint, but with different attachments of the leafsprings. For this topology, one set of leafsprings is connected to the fixed world and an intermediate body and the second set is connected between the intermediate body and end effector. Effectively, this leads to two spherical joints stacked in series (instead of parallel as with the PFL joint), each contributing to half of the motion. As the deflection per stage is halved, the stress levels in the flexures are reduced allowing for thicker leafsprings resulting in increased support stiffness. Furthermore, because the support stiffness decreases progressively non-linear with the deflection, halving the deflection leads to (far) less than half the stiffness loss over the range of motion. A schematic overview of the SFL joint is provided in Fig. 4.6

It has to be noted that the intermediate body is only constraint for translational motion and therefore contains three redundant rotational degrees of freedom (the intermediate body is three times underconstrained). For most flexure mechanisms, underconstrained intermediate bodies dramatically deteriorate support 


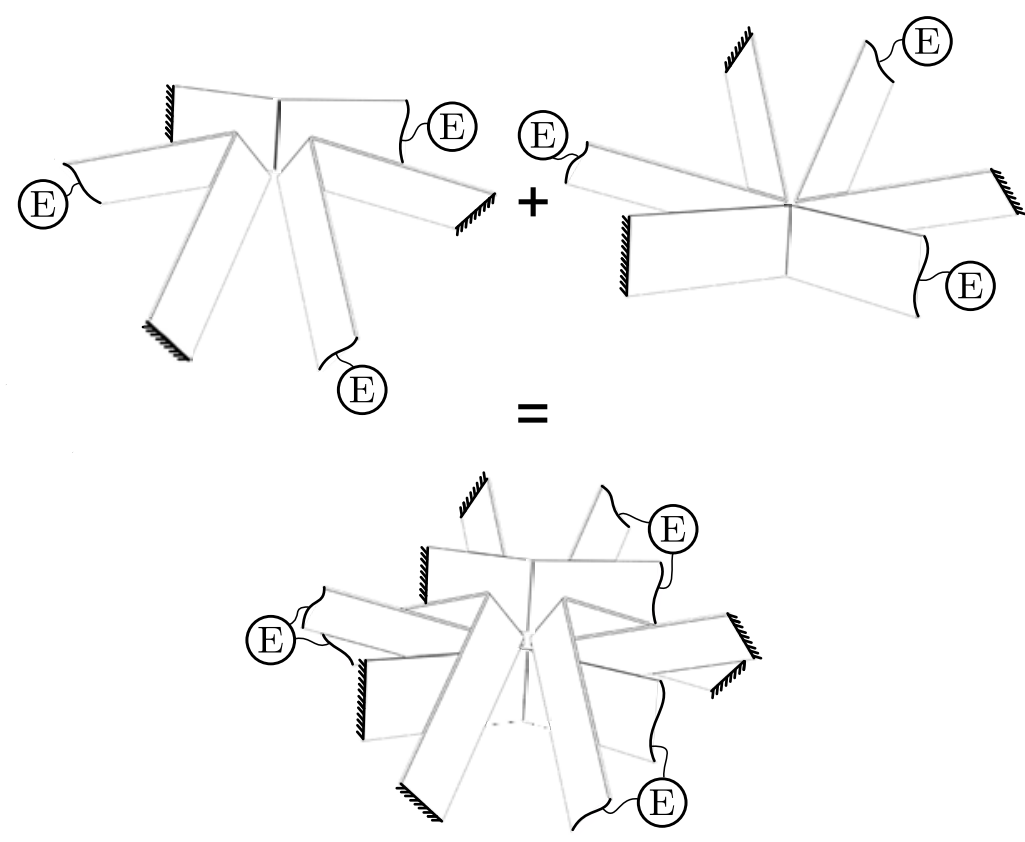

Figure 4.5: Parallel stacked Folded Leafspring based spherical joint topology $\left(\mathrm{PFL}_{3} \mathrm{r}_{3} \mathrm{p}_{1}\right)$ decomposed of two FL- $\mathrm{r}_{3} \mathrm{p}_{1}$ joints with "E" representing the connection with the end effector.

stiffness (particularly when the mechanism is in deflected state) due to coupling of external loads and the underconstrained degrees of freedom, such as the compounded parallel leafspring guidance without slaving [43].

In the SFL joint, the instant center of rotation of the intermediate body and the end effector coincide and do not vary much. Hence, external loads on the end effector do not result in reaction moments in the degrees of freedom of the intermediate body. Additionally, for the same reason, rotational motion of the intermediate body does not contribute to translational motion of the end effector. Due to this special property, support stiffness is not mitigated by the underconstrained intermediate body and the position of the end effector is not influenced by motion of the intermediate body. However, with respect to the dynamic behavior of the joint, the underconstrained intermediate body can result in unwanted vibrations in the system. Options to reduce vibrations of the intermediate body by adding damping and suggestions for eliminating the underconstraints are provided in section 4.8 


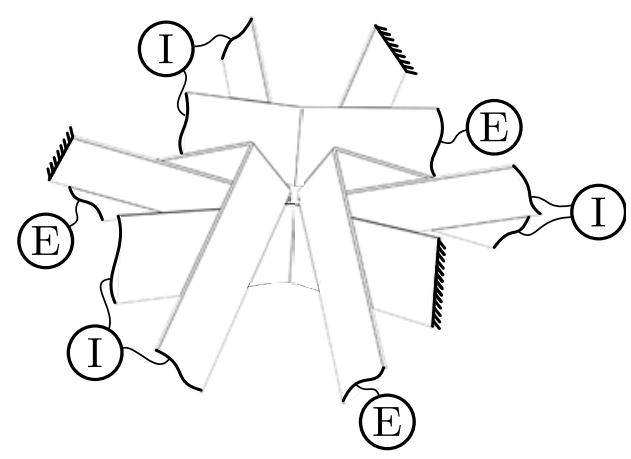

Figure 4.6: Serial stacked Folded Leafspring based spherical joint topology (SFL- $\left.\mathrm{r}_{3} \mathrm{p}_{1}\right)$ with "E" representing the connection with the end effector and "I" the connection with an intermediate stage

\subsection{Shape optimization}

\subsubsection{Optimization method}

For comparing the performance of the different joint topologies as described in section 4.4 the shape of each topology is optimized. For this optimization two cases are considered, one with a range of motion of 5 degrees tip-tilt and the other with 30 degrees tip-tilt. For optimization purposes, pan motion is disregarded as the support stiffness at maximum deflection is mainly affected by tip-tilt motion, which is substantiated by the validation in section 4.6 The shape optimization allows for the comparison of the maximum support stiffness which can be achieved for each topology and the influence of the range of motion on the performance. It has to be noted that the optimization results are specific for the selected case. However, it does provide useful insight on the typical stiffness characteristics of each topology and allows for a comparison between them.

The goal of the optimization procedure is to maximize the translational support stiffness along the pan axis at maximum tip-tilt angle when considering a range of motion of 30 degrees tip-tilt $\left(\omega_{t}=30^{\circ}\right.$ for $\left.0^{\circ}<\phi \leq 360^{\circ}\right)$ and 5 degrees tip-tilt $\left(\omega_{t}=5^{\circ}\right.$ for $\left.0^{\circ}<\phi \leq 360^{\circ}\right)$. Furthermore, build-space for the flexures is limited to a cylinder aligned with the $z$-axis with a radius of $75 \mathrm{~mm}$. For material we select tool steel (Stavax [99]) where we limit the maximum allowable stress due to deflection to $600 \mathrm{MPa}$ (yield strength: $1400 \mathrm{MPa}$ ).

To perform the shape optimization, a parametrized model of the spherical flexure joint with 6 design parameters is used, $\left[\theta_{1}, \psi, r, l, t, w\right]$, illustrated in Fig. 4.7 
Note that the folding angle $\psi$ is a parameter and hence not fixed to 90 degrees. Furthermore, when multiple sets of folded leafsprings are positioned in parallel under a different orientation angle, the orientation angle of the second set is provided by the additional design parameter $\theta_{2}$. In order to take into account all constraints (e.g. maximum stress, build-space and flexure collision) a NelderMead based optimization algorithm is adapted to include all boundary conditions and constraints [69]. Because it is hard to predict collision of the flexures due to 3D motion combined with large deformations, a collision detection algorithm was developed [67]. This algorithm allows for computationally efficient evaluation of the collision state by taking advantage of the typical slender aspect ratio and shape of the elastic members in flexure mechanisms, allowing for full utilization of the design freedom while ensuring collision free designs.

To numerically calculate the performance of a joint topology with a specific set of design parameters, the flexible multibody software SPACAR [45] is used. In SPACAR, all leafsprings are modeled with a series of interconnected nonlinear 3D finite beam elements which includes geometric non-linearities. Flexibility of these elements is naturally accounted for in the formulation owing to a specific choice of so-called discrete deformation modes. Therefore, only a limited number of elements is required to produce both fast and accurate results.

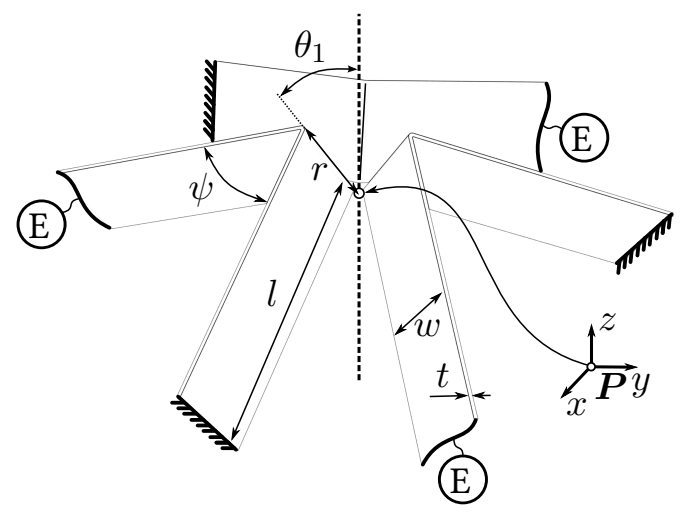

Figure 4.7: Parametrization of a FL- $r_{3} p_{1}$-joint with the instant center of rotation provided by $\boldsymbol{P}$ and "E" representing the connections with the end effector 


\begin{tabular}{lllllllllll}
$\begin{array}{l}\text { Topo- } \\
\text { logy }\end{array}$ & $\mathrm{r}$ & $\mathrm{p}$ & $l$ & $\begin{array}{l}r \\
(\mathrm{~mm})\end{array}$ & $\begin{array}{l}w \\
(\mathrm{~mm})\end{array}$ & $\begin{array}{l}\mathrm{(mm}) \\
(\mathrm{mm})\end{array}$ & $\begin{array}{l}\psi \\
(\mathrm{deg})\end{array}$ & $\begin{array}{l}\theta_{1} \\
(\mathrm{deg})\end{array}$ & $\begin{array}{l}\theta_{2} \\
(\mathrm{deg})\end{array}$ & $\begin{array}{l}K \\
(\mathrm{~N} / \mathrm{mm})\end{array}$ \\
\hline \hline $\mathrm{FL}$ & 3 & 1 & 53.2 & 60.4 & 59.9 & 0.96 & 72.9 & 24.2 & - & $3.7 \cdot 10^{4}$ \\
$\mathrm{FL}$ & 4 & 1 & 46.6 & 43.7 & 42.6 & 0.88 & 83.1 & 19.3 & - & $3.1 \cdot 10^{4}$ \\
$\mathrm{FL}$ & 5 & 1 & 48.7 & 41.7 & 40.3 & 0.96 & 67.8 & 20.3 & - & $3.6 \cdot 10^{4}$ \\
$\mathrm{FL}$ & 3 & 2 & 48.3 & 49.1 & 42.4 & 0.97 & 89.9 & 23.8 & 20.2 & $5.3 \cdot 10^{4}$ \\
$\mathrm{FL}$ & 4 & 2 & 41.6 & 39.6 & 38.2 & 0.75 & 84.2 & 25.0 & 12.5 & $5.6 \cdot 10^{4}$ \\
$\mathrm{FL}$ & 5 & 2 & 64.7 & 58.8 & 51.4 & 0.96 & 65.1 & 29.1 & 10.4 & $5.6 \cdot 10^{4}$ \\
\hline $\mathrm{PFL}$ & 3 & 1 & 70.7 & 73.9 & 64.4 & 0.93 & 73.5 & 8.0 & - & $4.4 \cdot 10^{4}$ \\
$\mathrm{PFL}$ & 4 & 1 & 45.5 & 40.5 & 39.1 & 0.86 & 76.1 & 22.0 & - & $5.3 \cdot 10^{4}$ \\
$\mathrm{PFL}$ & 5 & 1 & 41.8 & 33.4 & 32.4 & 0.85 & 67.0 & 21.7 & - & $5.5 \cdot 10^{4}$ \\
\hline $\mathrm{SFL}$ & 3 & 1 & 35.8 & 50.2 & 49.6 & 1.00 & 82.2 & 15.1 & - & $3.8 \cdot 10^{4}$ \\
$\mathrm{SFL}$ & 4 & 1 & 46.3 & 69.1 & 68.1 & 0.97 & 85.5 & 12.7 & - & $5.4 \cdot 10^{4}$ \\
$\mathrm{SFL}$ & 5 & 1 & 39.2 & 46.1 & 45.4 & 0.99 & 68.7 & 18.7 & - & $4.3 \cdot 10^{4}$ \\
\hline
\end{tabular}

Table 4.1: Optimization results for 5 degrees range of tip-tilt motion evaluated at maximum deflection angle with the design parameters according to Fig. 4.7

\subsubsection{Optimization results}

Results of the optimization considering 5 degrees of tip-tilt motion are provided in Table 4.1. When considering this small range of motion, it appears that increasing the number of rotational symmetric folded leafsprings does not necessary result in a proportional increase in support stiffness. Instead, even a small decrease in support stiffness is observed for some of the flexure topologies. This reduced support stiffness is presumably related to the increased probability of interference between the flexures. An increment in the number of flexures effectively reduces the available build-space for each folded leafspring, which reduces the design freedom and subsequently its support stiffness.

Furthermore, increasing the number of flexure sets with a different orientation angle for the FL joint topology does have a positive effect on the support stiffness, increasing support stiffness up to almost a factor of two. A similar performance is obtained with the serial (SFL) and parallel (PFL) stacked joint topologies, which shows no distinct advantage over the FL joint.

Results of the optimization considering 30 degrees of tip-tilt motion is provided in Table 4.2 For this larger range of motion, increasing the number of rotational symmetric folded leafsprings or the number of parallel flexure sets results in a 


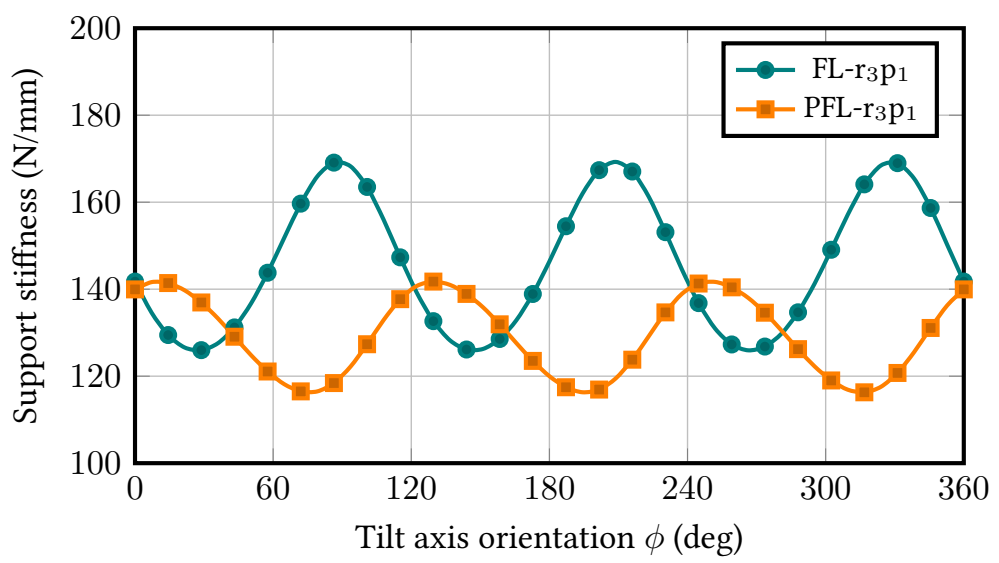

Figure 4.8: Support stiffness of optimized FL-r $\mathrm{r}_{3} \mathrm{p}_{1}$ and PFL-r $\mathrm{r}_{3} \mathrm{p}_{1}$ joint at $\omega_{t}=30^{\circ}$ for a tilt axis orientation of $0^{\circ} \leq \phi<360^{\circ}$

reduction in support stiffness for all joint topologies. The strongest reduction is observed for the FL and PFL joint topologies, which suffer from a reduction in support stiffness up to $90 \%$ for increased number of flexures. This decrease can again be related to the increased probability of interference between the flexures for an increasing flexure count, which influence is even bigger for this larger range of motion.

Furthermore, the FL- and PFL joint topologies provide a similar level of support stiffness. It has to be noted that the PFL joint topology does provide a reduced dependency of the support stiffness on the orientation of the tilt axis $\phi$. This dependency is visualized in Fig. 4.8, where it can be observed that support stiffness for the FL- $\mathrm{r}_{3} \mathrm{p}_{1}$ joint is strongly dependent on the orientation of the tilt axis. By adding the secondary set of folded leafsprings in the PFL- $\mathrm{r}_{3} \mathrm{p}_{1}$ joint with the rotational offset of $180 / \mathbf{r}_{n}$ degrees, low support stiffness of the first set is compensated with high support stiffness of the second set. Hereby, the variation in support stiffness as function of the orientation of the tilt axis is reduced by approximately $40 \%$.

At last, the SFL joint topology provides a large improvement in performance with almost a factor 5 increase in support stiffness with respect to the FL and PFL joint topologies when considering the 30 degrees of tip-tilt motion. Due to the serial stacking of the joints, the deformations of the individual flexures are smaller resulting in a smaller decrease in support stiffness over the range of motion. Furthermore, the smaller deformations allow for shorter, wider and/or thicker leafsprings when considering the same stress levels, providing an additional gain 


\begin{tabular}{lllllllllll}
$\begin{array}{l}\text { Topo- } \\
\text { logy }\end{array}$ & $\mathrm{r}$ & $\mathrm{p}$ & $l$ & $\begin{array}{l}r \\
(\mathrm{~mm})\end{array}$ & $\begin{array}{l}w \\
(\mathrm{~mm})\end{array}$ & $\begin{array}{l}t \\
(\mathrm{~mm})\end{array}$ & $\begin{array}{l}\psi \\
(\mathrm{mm})\end{array}$ & $\begin{array}{l}\theta_{1} \\
(\mathrm{deg})\end{array}$ & $\begin{array}{l}\theta_{2} \\
(\mathrm{deg})\end{array}$ & $\begin{array}{l}K \\
(\mathrm{~N} / \mathrm{mm})\end{array}$ \\
\hline \hline FL & 3 & 1 & 74.2 & 42.3 & 39.0 & 0.49 & 86.9 & 43.2 & - & 126 \\
FL & 4 & 1 & 83.7 & 29.8 & 23.9 & 0.47 & 51.3 & 47.9 & - & 46 \\
FL & 5 & 1 & 82.4 & 43.4 & 42.7 & 0.40 & 37.8 & 53.5 & - & 40 \\
FL & 3 & 2 & 75.6 & 33.4 & 23.8 & 0.50 & 82.2 & 53.2 & 43.6 & 120 \\
FL & 4 & 2 & 75.3 & 20.1 & 8.7 & 0.48 & 67.8 & 43.0 & 29.8 & 38 \\
FL & 5 & 2 & 61.7 & 49.0 & 22.5 & 0.20 & 70.0 & 46.9 & 33.3 & 10 \\
\hline PFL & 3 & 1 & 78.6 & 18.6 & 15.9 & 0.57 & 95.4 & 39.6 & - & 116 \\
PFL & 4 & 1 & 58.7 & 16.1 & 12.8 & 0.37 & 70.7 & 32.3 & - & 96 \\
PFL & 5 & 1 & 72.5 & 29.7 & 13.5 & 0.32 & 60.8 & 33.3 & - & 30 \\
\hline SFL & 3 & 1 & 33.5 & 26.0 & 22.3 & 0.41 & 96.7 & 40.0 & - & 568 \\
SFL & 4 & 1 & 53.6 & 22.0 & 20.5 & 0.64 & 78.2 & 30.0 & - & 560 \\
SFL & 5 & 1 & 62.3 & 25.7 & 21.5 & 0.64 & 63.8 & 24.8 & - & 450 \\
\hline
\end{tabular}

Table 4.2: Optimization results for 30 degrees range of tip-tilt motion evaluated at maximum deflection angle with the design parameters according to Fig. 4.7

in performance. Especially for this large range of motion, this leads to a large increase of support stiffness. Compared to traditional spherical flexure joints which allow for a similar range of motion, for example the stacked arrangement of three cruciform hinges which provide a support stiffness of approximately $10 \mathrm{~N} / \mathrm{mm}$ [15 90], an increase in support stiffness of more than a factor of 50 is obtained.

\subsection{Spherical joint prototype}

\subsubsection{Prototype design}

Based on the optimization results, a prototype of the best performing spherical joint topology for a range of motion of \pm 30 degrees tip-tilt is constructed (the SFL- $\mathrm{r}_{3} \mathrm{p}_{1}$ joint). Additional to the \pm 30 degrees of tip-tilt motion, the joint allows for \pm 10 degrees of rotation along the pan axis.

A photograph of the prototype in undeflected and deflected state is provided in Figs. 4.9 and 4.10 . This prototype is constructed from tool steel flexures of $0.4 \mathrm{~mm}$ thickness combined with three aluminum frame bodies (the base and end effector 


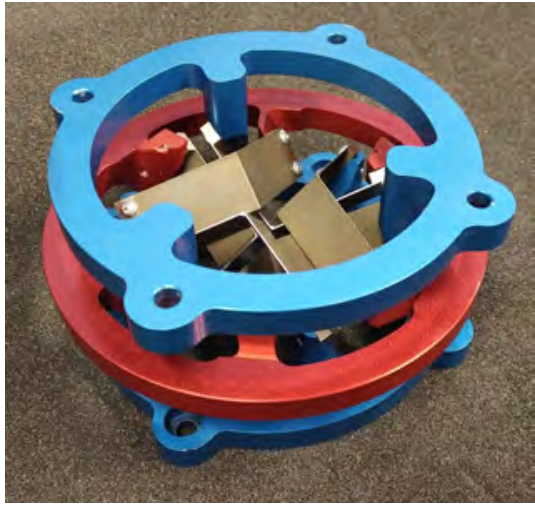

(a) Perspective view

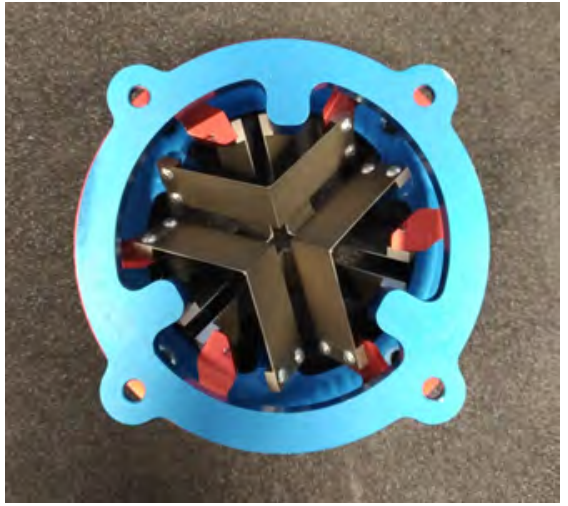

(b) Top view

Figure 4.9: Photographs of the SFL- $\mathrm{r}_{3} \mathrm{p}_{1}$ joint prototype.

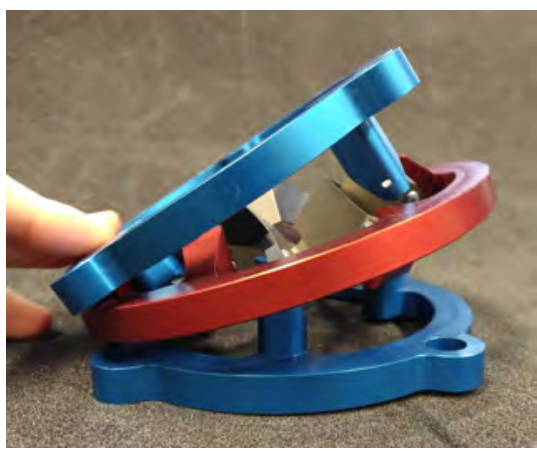

(a) $\phi=0^{\circ}, \omega_{t}=30^{\circ}$

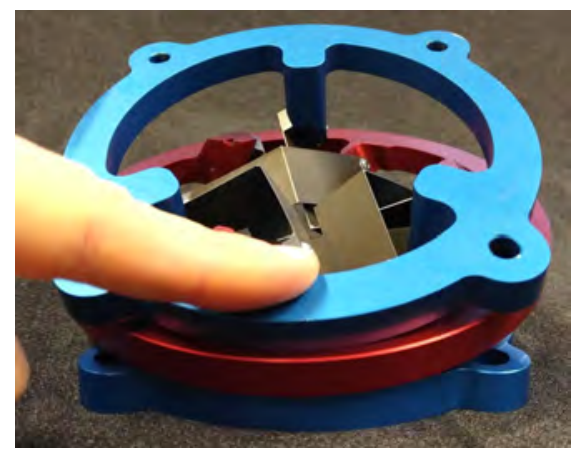

(b) $\phi=90^{\circ}, \omega_{t}=30^{\circ}$

Figure 4.10: Photographs of the SFL- $\mathrm{r}_{3} \mathrm{p}_{1}$ joint prototype in deflected state.

anodized in blue, the intermediate body anodized in red). A full overview of the design parameters is provided in Table 4.3 which provides a simulated support stiffness of $465 \mathrm{~N} / \mathrm{mm}$ along the pan axis at the maximum tip-tilt angle.

\subsubsection{Prototype performance}

In Fig. 4.11 4.14 the simulated support stiffness, maximum stress in the flexures and shift of the pivot of the spherical joint are presented as function of tip-tilt and pan motion. The orientation of the tilt axis is kept constant as it has only minor influence on the results. Therefore, all results are presented for an orientation of the tilt axis of $\phi=0$ degrees. 
Table 4.3: Design parameters of the SFL- $\mathrm{r}_{3} \mathrm{p}_{1}$ joint prototype

\begin{tabular}{ll} 
Design parameter & Value \\
\hline \hline$l$ & $36.8 \mathrm{~mm}$ \\
$r$ & $24.8 \mathrm{~mm}$ \\
$w$ & $20 \mathrm{~mm}$ \\
$t$ & $0.4 \mathrm{~mm}$ \\
$\psi$ & $105^{\circ}$ \\
$\theta_{1}$ & $40.0^{\circ}$ \\
\hline
\end{tabular}

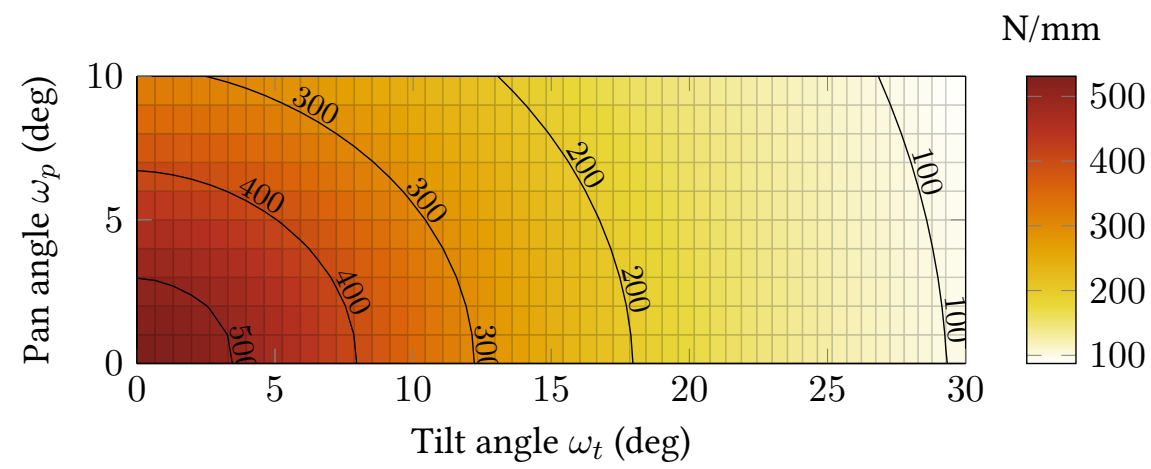

Figure 4.11: Translational support stiffness evaluated along the tilt axis $\left(\phi=0^{\circ}\right)$

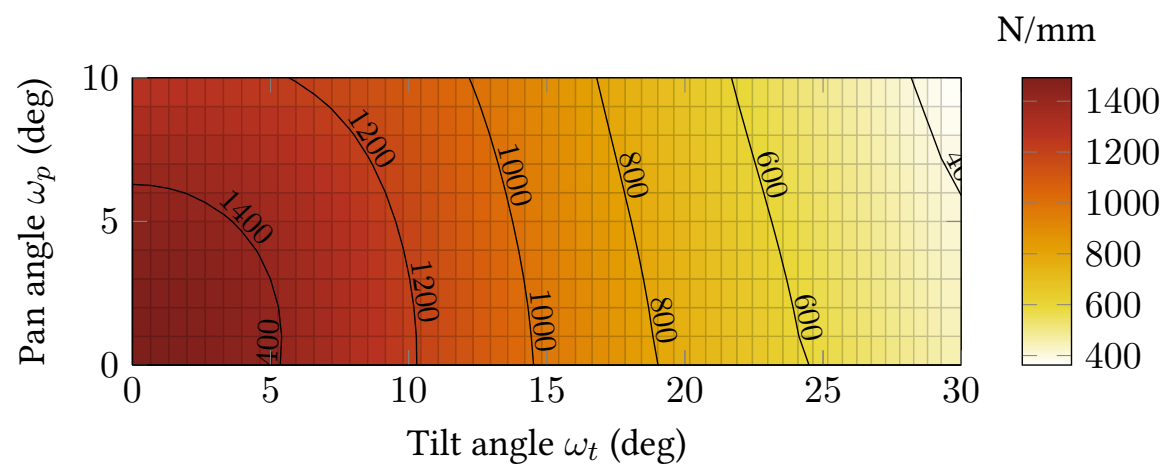

Figure 4.12: Translational support stiffness evaluated along the pan axis $\left(\phi=0^{\circ}\right)$ 


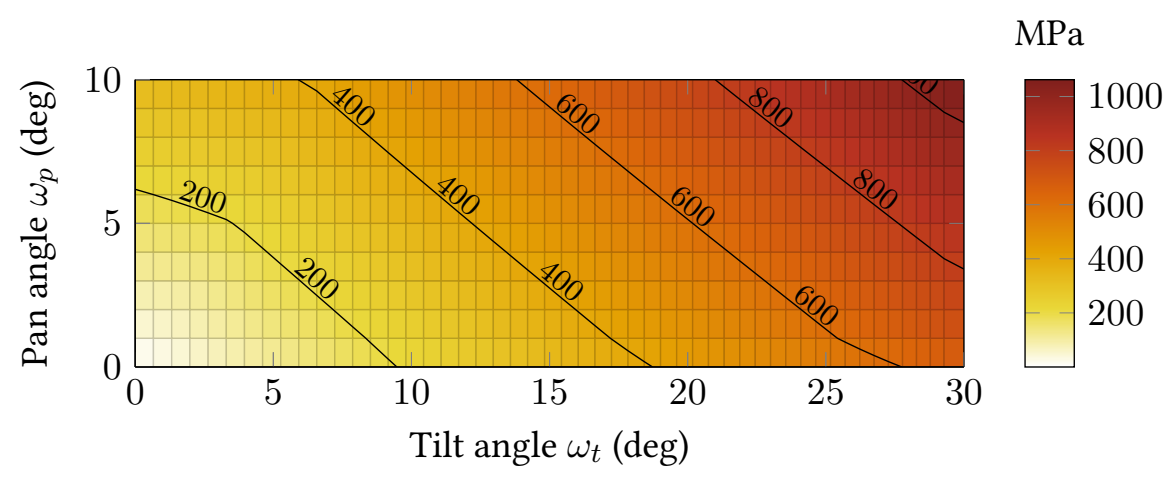

Figure 4.13: Maximum von Mises stress $\left(\phi=0^{\circ}\right)$

From these results it can be concluded that the minimum support stiffness is encountered at maximum tilt angle. At this maximum tilt angle, additional pan motion has only a minor influence on the support stiffnesses (Fig. 4.11 and 4.12). This justifies the chosen optimization criteria where the minimum support stiffness as function of the tilt angle is selected for the optimization objective where pan motion is disregarded. For small deflections, a support stiffness of $1490 \mathrm{~N} / \mathrm{mm}$ along the pan axis and $530 \mathrm{~N} / \mathrm{mm}$ along the tilt axis are obtained. For larger deflections, for example 30 degrees tip-tilt, support stiffness decreases to $465 \mathrm{~N} / \mathrm{mm}$ along the pan axis and $98 \mathrm{~N} / \mathrm{mm}$ along the tilt axis.

With respect to the stress in the flexures, both tip-tilt and pan motion have a similar contribution. Overall, maximum stress is mainly depended on the total deflection angle provided by $\omega_{t}+\omega_{p}$ (Fig. 4.13). As a results of this, the maximum tilt and pan angle cannot be obtained simultaneously when considering a maximum stress of $600 \mathrm{MPa}$. If simultaneous motion is required in both directions, this could be taken into account in the optimization procedure by reducing the constraint on the maximum stress. As tilt and pan motion have a similar contribution to the stress levels, the stress under combined tilt and pan motion can be estimated by extrapolating the stress values.

Furthermore, shift of the pivot of the joint is provided in Fig. 4.14 which is defined as the traveled distance of the node, connected to the end effector, which is initially positioned in the instant center of rotation. When the pivot shift is compared to the traditional cross-spring pivot with a range of motion of $30 \mathrm{de}-$ grees ( $>1 \mathrm{~mm}$ shift of the pivot axis for typical dimensions of the cross-spring pivot [35]), pivot shift of the spherical joint is relatively small $(<0.45 \mathrm{~mm})$. Furthermore, shift of the pivot behaves non-linearly for increasing tip-tilt and pan motion. Due to this non-linear behavior, pivot shift is almost absent for small 


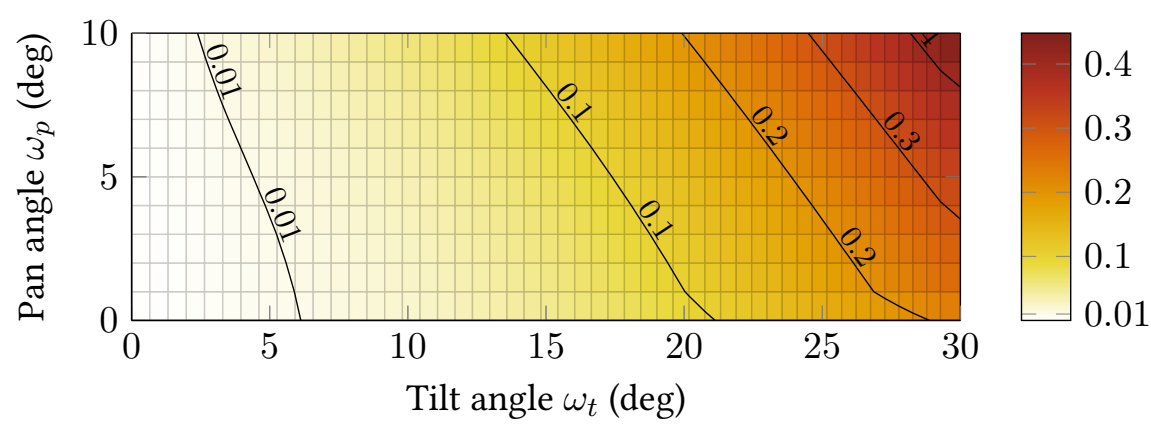

Figure 4.14: Pivot shift $\left(\phi=0^{\circ}\right)$

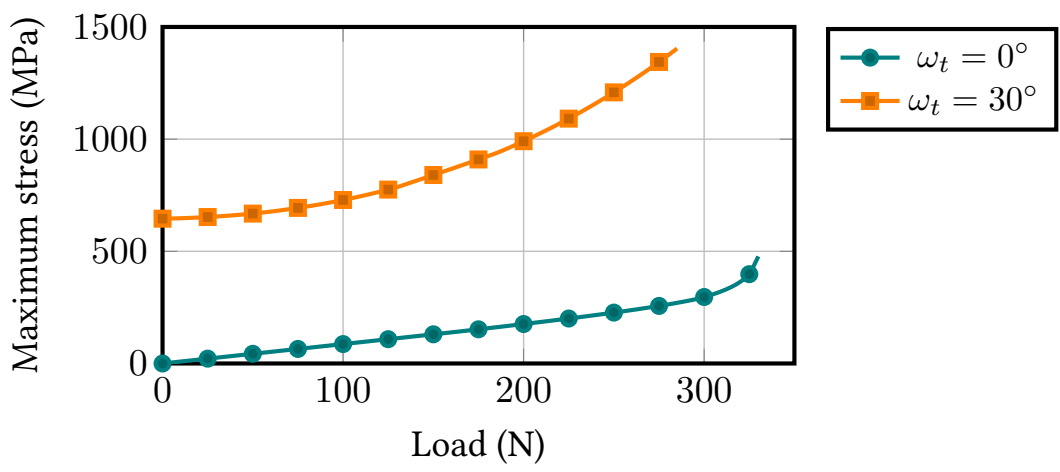

Figure 4.15: Stress (von Mises) as function of load applied along the pan axis

deflections but increases more strongly for larger deflection angles.

At last, the effect of load on the support stiffness and stress is provided in Fig.4.15 and 4.16 respectively. Load is applied along the pan axis and increased until the system has buckled or until the yield stress of the material is exceeded. For zero tilt motion $\left(\omega_{t}=0^{\circ}\right)$, the load capacity is limited to $330 \mathrm{~N}$ by buckling at which the support stiffness shows a strong sudden decrease (Fig. 4.15). For larger tilt angles $\left(\omega_{t}=30^{\circ}\right)$, a more gradual decrease in stiffness is observed due to the deflected state of the flexures. For this case, load capacity is limited by stress in the flexures, which exceeds the yield stress at a load of $290 \mathrm{~N}$ (Fig. 4.16). 


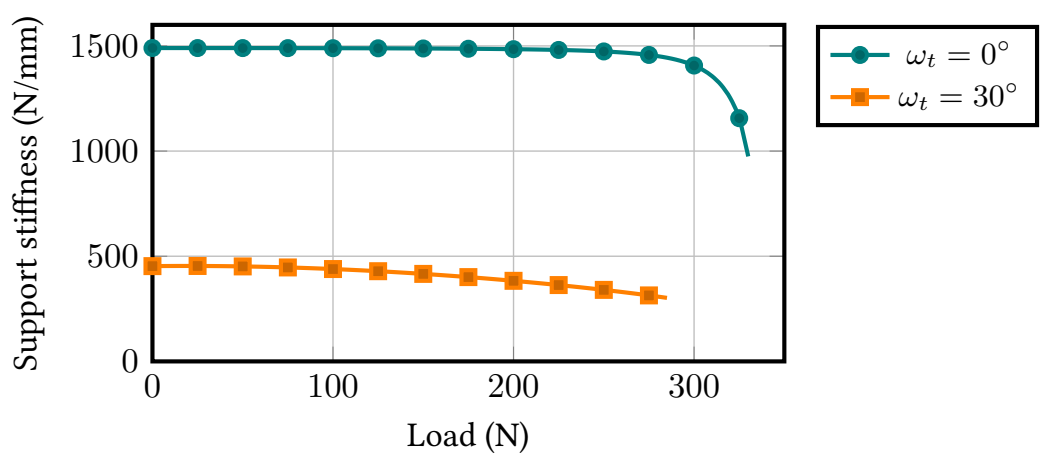

Figure 4.16: Support stiffness along the pan axis as function of load applied along the pan axis.

\subsection{Experimental validation}

\subsubsection{Experimental setup}

For validation of the support stiffness and to assess the dynamic behavior of the underconstrained intermediate body, an experimental validation is conducted. The experimental setup is shown in Fig. 4.17. It consists of a tilting platform which allows for rotation of the joint to the desired tilt angle. The tilt angle can be read from a protractor. At the other end of the joint, the spherical joint is connected to a "suspended platform" which is guided with a single leafspring to prevent rotations in the degrees of freedom when loaded (the leafspring exactly constraints the remaining three degrees of freedom of the spherical joint). Load is applied by a single wire flexure which is connected to a force sensor (MEKM10z, range: $200 \mathrm{~N}$, accuracy: $<0.2 \mathrm{~N}$ ) to measure the applied load. The other end of the wire flexure is connected to a micrometer attached to a linear stage in order to gradually apply the required load. Additionally, displacement of the "suspended platform" caused by the applied load is measured with a capacitive sensor (Lion Precision C8-3.2, range: $1250 \mu \mathrm{m}$, accuracy: $<0.1 \mu \mathrm{m}$ ) to evaluate the stiffness along the pan axis. The applied load, force sensor and displacement sensor are all positioned inline directly underneath the center of rotation of the spherical joint to comply with the Abbe principle [1]. 


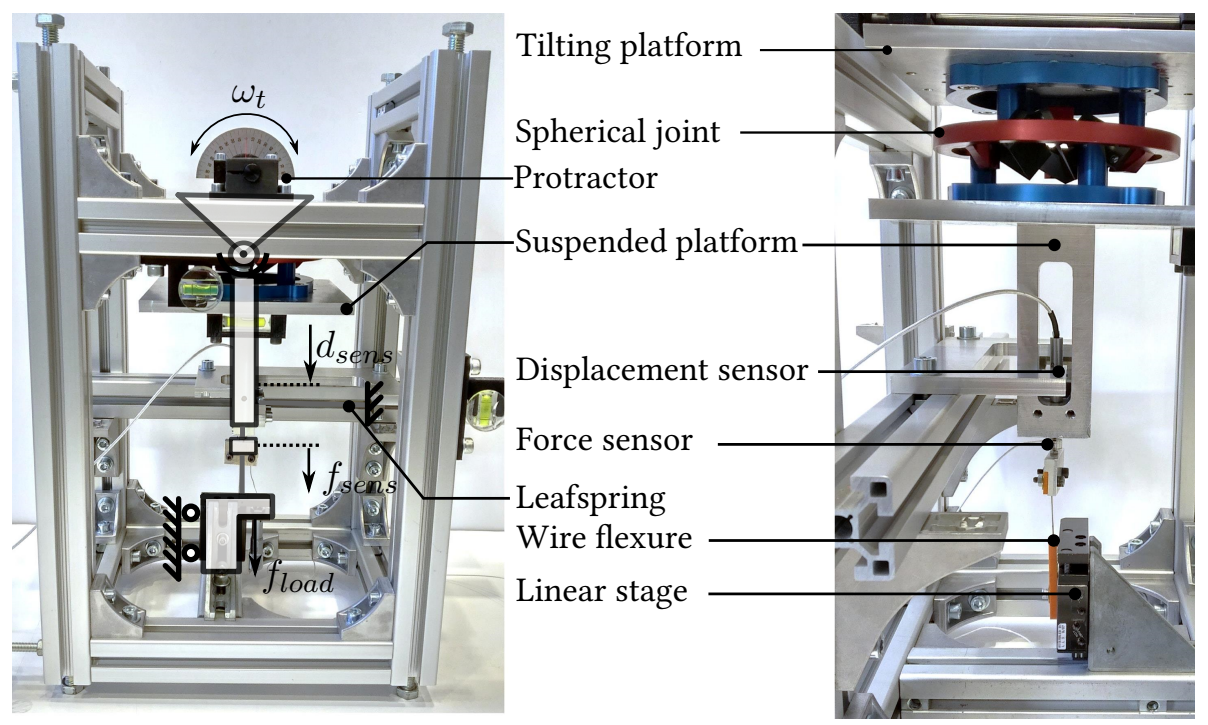

Figure 4.17: Experimental setup for validating the spherical joint properties (left: front view with schematic drawing, right: side view)

\subsubsection{Stiffness validation}

The support stiffness along the pan axis is tested by applying an alternating load between $5 \mathrm{~N}$ and $15 \mathrm{~N}$ and continuously measuring the applied force $\left(f_{\text {sens }}\right)$ and the resulting displacement $\left(d_{\text {sens }}\right)$ with a sample rate of $500 \mathrm{~Hz}$. Minimum tensile load of $5 \mathrm{~N}$ is maintained to prevent buckling of the wire-flexure and to minimize hysteresis effects in the force sensor. An example of the measurement of the support stiffness at 30 degrees tilt is provided in Fig. 4.18 where the load is alternated 5 times in 20 seconds (approximately 10000 samples). By using a linear fit through the obtained data, the support stiffness can be evaluated. This test is repeated for different tilt angles in order to obtain the support stiffness as function of the tilt angle.

An overview of the measured and simulated stiffness as function of the tilt angle are provided in Fig. 4.19 Especially for small tilt angles, the measured stiffness is substantially lower than the simulated stiffness obtained from the SPACAR simulation. This difference in support stiffness can be related to additional compliance introduced by the frame parts that connect the leafsprings. During the design of the spherical joint, care is taken to ensure high stiffness of the frame parts. Despite this, limitations on the design freedom imposed by the avoidance of collision of the flexures and frame parts over the range of motion inherently 


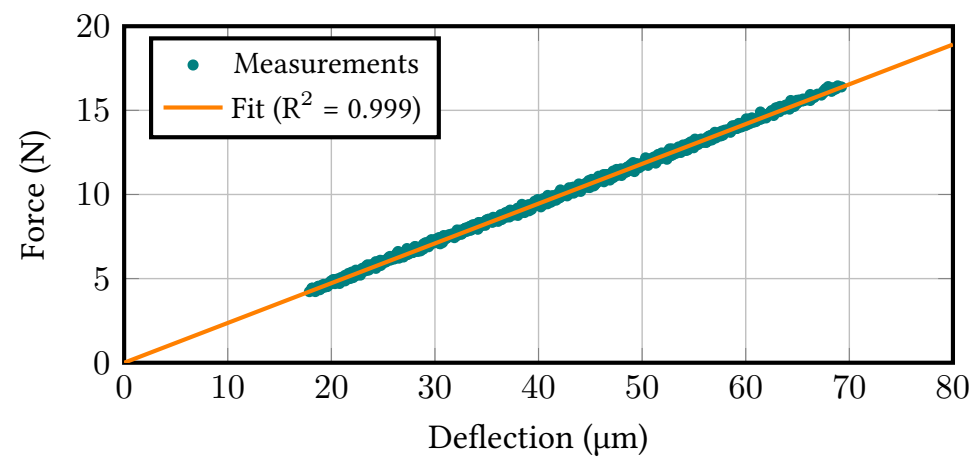

Figure 4.18: Measurement results for testing support stiffness at 30 degrees tilt angle. A stiffness of $236 \mathrm{~N} / \mathrm{mm}$ is obtained from a linear fit through the data with $\mathrm{R}^{2}=0.999$.

limit the stiffness of the frame. Therefore, the additional compliance of the frame has to be taken into account to accurately assess the support stiffness of the entire joint.

For evaluating the effect of frame compliance on the overall support stiffness, a FEM analysis (Solidworks Simulation) considering zero tilt angle is conducted, both for a flexible and rigid frame. The overall stiffness of the joint appeared to be $1.3 \cdot 10^{3} \mathrm{~N} / \mathrm{mm}$ considering rigid frame parts. Stiffness decreased to 3.1 . $10^{2} \mathrm{~N} / \mathrm{mm}$ for a realistic representation of the frame. From these values an approximate of the frame stiffness can be computed, assuming the flexures and frame stiffness are in series. Hence, the equivalent frame stiffness is given by

$$
K_{f r}=\frac{1}{\frac{1}{3.1 \cdot 10^{2}}-\frac{1}{1.3 \cdot 10^{3}}}=4.1 \cdot 10^{2} \mathrm{~N} / \mathrm{mm}
$$

By adding this equivalent frame stiffness in series to the obtained stiffness obtained from SPACAR simulations, an approximate stiffness can be estimated. When frame compliance is added to the simulations, a good match is obtained between experiment and simulations showing a support stiffness of $215 \mathrm{~N} / \mathrm{mm}$ at the maximum tilt angle of 30 degrees.

Additional to the evaluation of the support stiffness for small loads, stiffness under high load and the load capacity is tested. For this purpose, load has been increased up to approximately $150 \mathrm{~N}$ at a tilt angle of 30 degrees at which a displacement of $0.65 \mathrm{~mm}$ was measured. This indicates no significant decrease in support stiffness even when high loads are applied and partially confirms the load capacity as indicated in section 4.6 Higher loads have not been tested due 


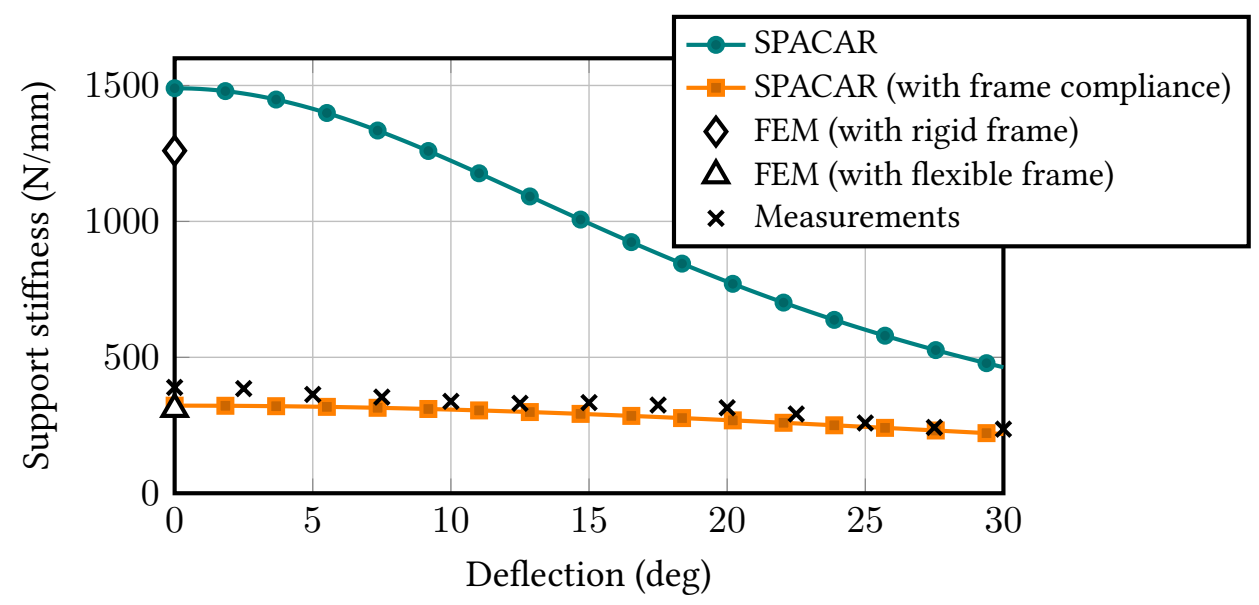

Figure 4.19: Experimental validation of the translational support stiffness measured along the pan axis

to the limited range of the force sensor and to prevent plastic deformations of the flexures which would compromise other measurements.

\subsubsection{Intermediate body dynamics}

As discussed in section 4.4 .3 the intermediate body of the SFL joint is underconstrained which could potentially influence the dynamic behavior of the system. If there is a slight distance between the remote centers of rotation, vibrations of this intermediate body could result in unwanted translational motion on the end effector. Secondly, vibrations of the intermediate body could be undesirable as it potentially induces vibrations in the rest of the system.

The effect of intermediate body vibrations (without damper) are evaluated by triggering the intermediate body by deflecting it approximately 5 degrees in tilt direction and releasing it. Next, the displacement along the pan axis caused by vibrations of the internal body is measured with the displacement sensor. The wire flexure is removed to allow for free motion along the pan axis. The results are shown in Fig. 4.20 where the intermediate body is released at approximately $t=0.5 \mathrm{~s}$.

From these results it can be concluded that the parasitic motion of the end effector caused by intermediate body vibrations is small, i.e. within a few micrometers, which is expected as ideally rotational motion of the intermediate body is decoupled from motion of the end effector due to the coinciding rotation cen- 


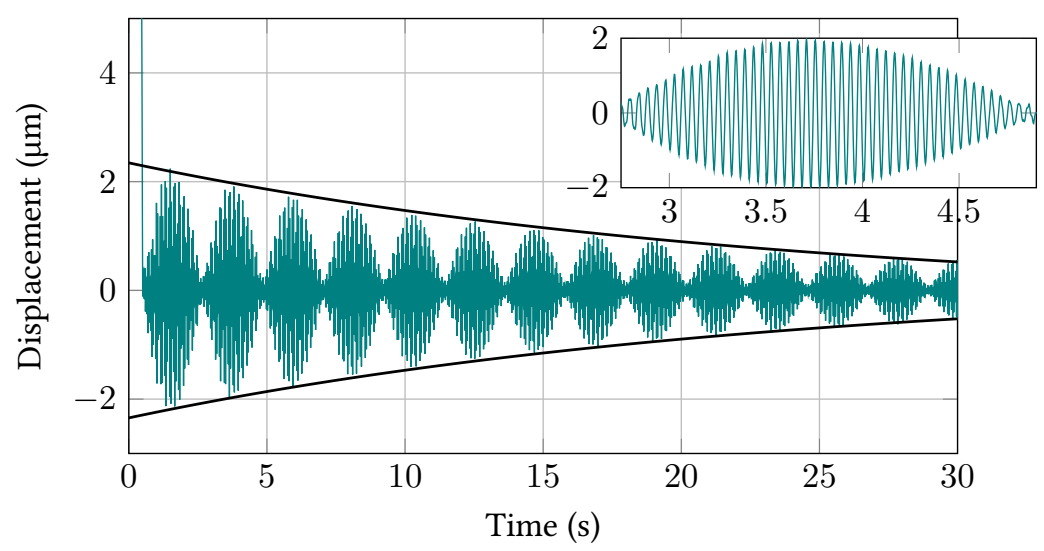

Figure 4.20: Displacement of the end effector measured along the pan axis caused by intermediate body vibrations with zoomed in section in the top right corner. Fitted exponential curve to assess damping properties: $f(t)=2.526 e^{-0.0426 t}$.

ters. Furthermore, a beat frequency can be identified in this time signal, which is caused by interference between two vibration modes with slightly different frequencies. This results from the two underconstrained degrees of freedom in tip-tilt direction which should have a similar eigenfrequency. This phenomenon can be identified more clearly from the FFT data obtained from the time signal, provided at Fig.4.21 The eigenfrequencies of the underconstrained tip-tilt modes can be identified at $21.73 \mathrm{~Hz}$ and $22.20 \mathrm{~Hz}$. This results in a beat frequency of $0.47 \mathrm{~Hz}$ which corresponds to the beat frequency observed in the time signal. Furthermore, the third vibration mode (pan motion of the intermediate body) is seen at $26.5 \mathrm{~Hz}$.

In order to assess the damping ratio of the intermediate body vibrations, an exponential curve $\left(f(t)=\alpha e^{-\zeta \omega_{n} t}\right)$ is fitted through the peaks of the time data (Fig. 4.20). Hereby, the damping ratio of this vibration mode can be evaluated based on the logarithmic decrement of the magnitude of the vibration. The damping ratio $\zeta$ for the undamped intermediate body equals to $\zeta \approx 0.0003$. As only material- and air-damping is existent, damping is low and vibrations are barely damped. 


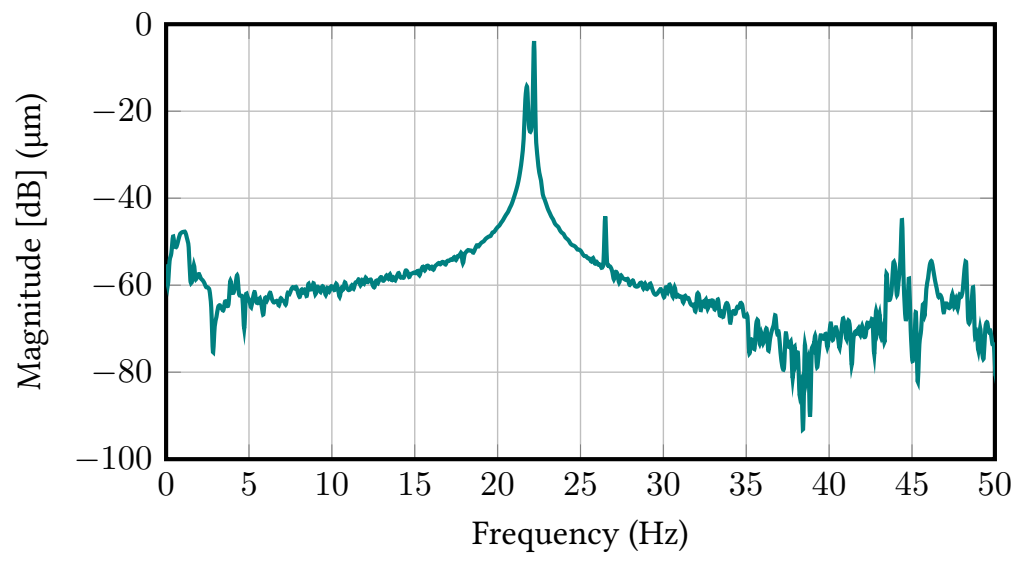

Figure 4.21: Fast Fourier transform of end effector vibrations caused by intermediate body vibrations

\subsection{Suppressing intermediate body vibrations}

In this section two options are discussed for reducing vibrations of the underconstrained intermediate body. The first option makes use of eddy current damping to suppress vibrations. The second option consists of a slaving mechanism to eliminate the internal underconstraints.

\subsubsection{Adding damping}

In order to reduce vibrations of the intermediate body, additional damping can be added. For this purpose, a contactless damper based on the eddy current principle is well suited as it does not rely on a physical contact between the joint and damper. Damping is then provided by a conductor (i.e. the aluminum intermediate body) which moves through a magnetic field inducing eddy currents in the conductor [55, 91]. To test the effect of an eddy current damper, a damper is manufactured consisting of a total of 140 neodymium magnets. A photograph of the prototype with eddy current damper is shown in Fig. 4.22 All magnets are positioned at close distance of the intermediate body in order to maximize the eddy current effect.

The resulting time response of the intermediate body vibrations with the additional eddy current damper is shown in Fig. 4.23 For the damped intermediate body, the exponential decay obtained from the fitted exponential curve provides a damping ratio of approximately $\zeta \approx 0.0021$, which is an increase of a factor 


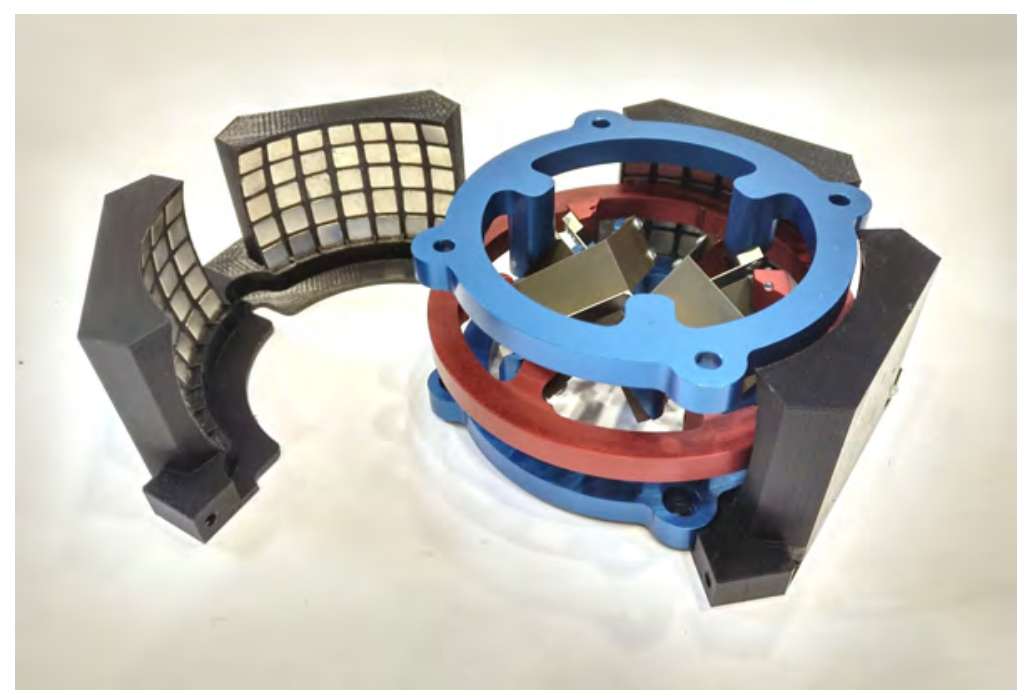

Figure 4.22: Spherical SFL- $\mathrm{r}_{3} \mathrm{p}_{1}$ joint with (partially detached) eddy current damper.

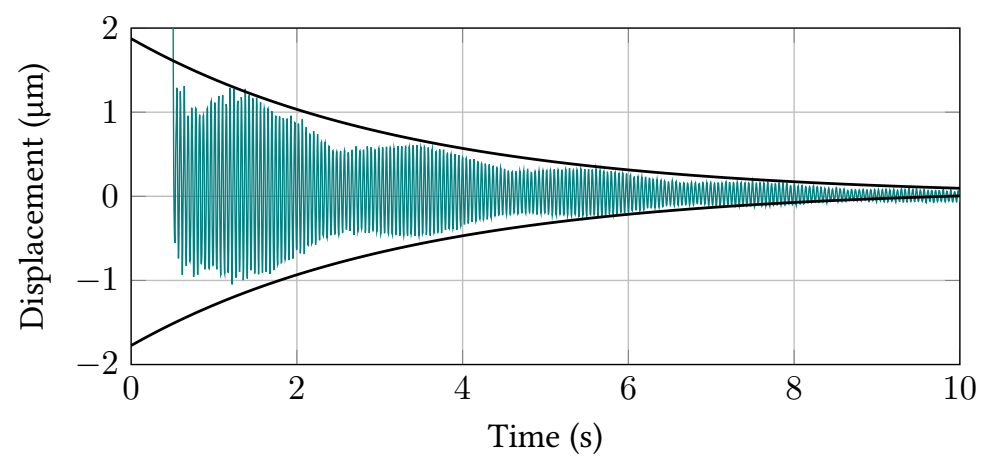

Figure 4.23: Displacement of the end effector measured along the pan axis caused by intermediate body vibrations with additional magnetic damper. Fitted curve: $f(t)=$ $1.874 e^{-0.298 t}$.

seven in damping. The eddy currents clearly help reducing internal vibrations. A further reduction can be obtained by strengthening the magnetic field or by reducing the air gap between the magnets and intermediate body.

\subsubsection{Adding a slaving mechanism}

An alternative option to reduce vibrations of the intermediate body is to add an additional slaving mechanisms, which then eliminates the underconstraints. 


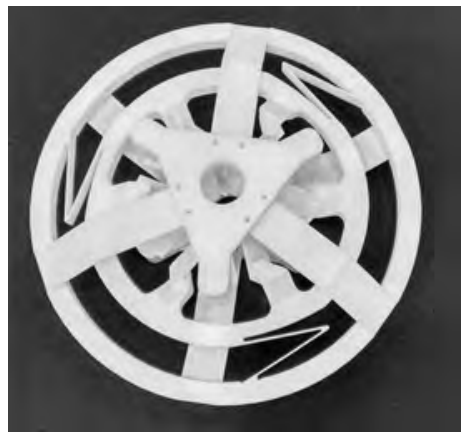

(a)

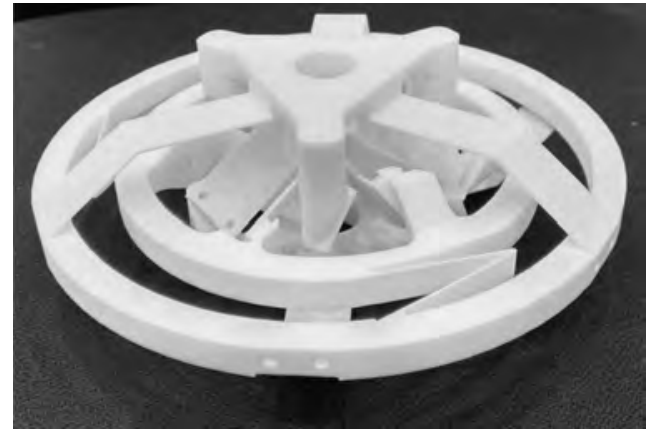

(b)

Figure 4.24: Prototype of a Spherical SFL- $\mathrm{r}_{3} \mathrm{p}_{1}$ joint with underconstrained eliminator manufactured from Nylon by selective laser sintering with (a) top view and (b) perspective view.

This section provides some ideas and design suggestions in order to eliminate the underconstraints of the intermediate body in the serial stacked SFL joint.

Solutions for coupling motion of the end effector to an intermediate stage with a two-to-one slaving mechanism for single degree of freedom flexure systems have been provided in literature [9, 12, 38, 44], typically utilizing an auxiliary body acting as a lever ensuring the required ratio in motion. However, exploiting this method for a multi-degree-of-freedom system is less trivial due to the additional degrees of freedom of the auxiliary body. An attempt for coupling motion of the intermediate body with an auxiliary embodiment is presented in Fig. 4.24 and 4.25. which schematically shows the working principle and picture of an additive manufactured prototype in Nylon. The auxiliary embodiment is connected to the intermediate body with three folded leafsprings which ensures coupling of the tilt motion of both bodies. Additionally, the auxiliary body is connected with three folded leafsprings to both the end effector and base to provide the require two-to-one ratio in motion between the end effector and intermediate body. It has to be noted that the auxiliary embodiment only couples tip-tilt motion in the two-to-one ratio and does not eliminate the underconstrained pan motion of the intermediate body. However, as the frequency of the pan mode is typically higher (especially when considering the additional stiffness of the slaving mechanism) with a relatively small amplitude, this remaining underconstrained degree of freedom could be acceptable. Additionally it has to be noted that pan motion of the end effector is constrained by the slaving mechanism effectively creating a universal joint instead of a spherical joint. 


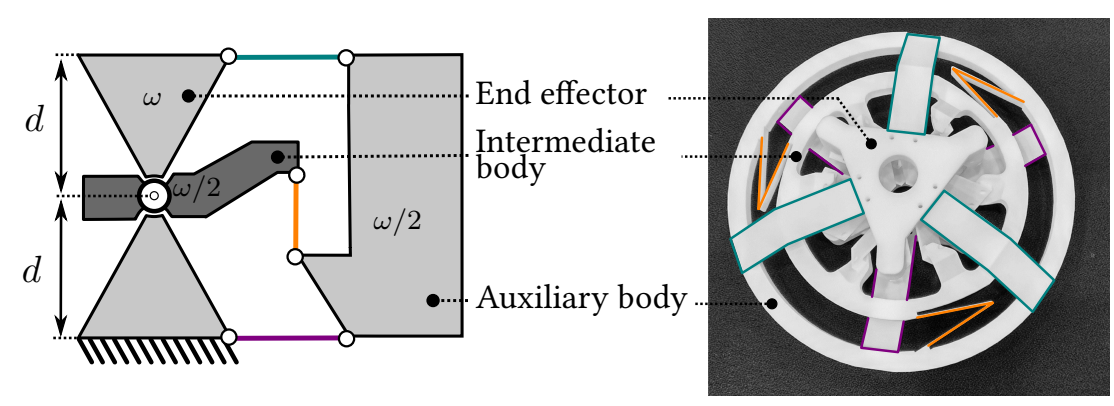

Figure 4.25: Schematic illustration of the working principle of the spherical SFL- $\mathrm{r}_{3} \mathrm{p}_{1}$ joint with underconstrained eliminator. $\omega$ indicates the rotation angle of the end effector.

\subsection{Conclusion}

A large range of motion spherical flexure joint can be obtained by using a topology with three or more folded leafsprings in parallel, of which all folding lines intersect at a single point. Especially when considering a large range of motion, increasing the number of folded leafsprings does not guarantee an increased level of support stiffness in the load-bearing directions, which is caused by the increased risk of collision between the flexures. The highest level of support stiffness can be obtained by effectively stacking two spherical joints in series, each having three folded leafsprings in parallel. With this design the deformations of the flexures are halved allowing for stiffer flexures for the same level of stress. This results in a significant increase in support stiffness, although it comes at the cost of an underconstrained intermediate body which could require additional damping or a slaving mechanism for some applications, for which several options are presented. Structural optimizations on a diverse set of flexure topologies have resulted in a flexure-based spherical joint which allows for \pm 30 degrees tiptilt and \pm 10 degrees pan motion. At maximum deflection, this joint maintains a support stiffness of over $200 \mathrm{~N} / \mathrm{mm}$, which is more then an order of magnitude higher than the current state-of-the-art spherical flexure joints with similar range of motion. Furthermore, load capacity is unmatched $(290 \mathrm{~N}$ at 30 degrees tip-tilt) and pivot shift remains below $0.5 \mathrm{~mm}$ for a combined 30 degrees tiptilt and 10 degrees pan motion. Experimental validations verify the simulated performance and confirm the high support stiffness and load capacity over the entire range of motion 


\title{
Large stroke high off-axis stiffness two degree of freedom universal flexure joint
}

\begin{abstract}
Universal two degree of freedom flexure joints are often used for parallel manipulators or applications which demand transmission of torque. However, contemporary flexure-based universal joints are limited to small deflection angles caused by their significant loss of stiffness in support directions when deflected or are initially already compliant in the intended support directions. In this paper, a new concept for a high performance large stroke universal flexure joint is presented which can maintain a high level of support stiffness in both translational and torsional directions over its total range of motion of \pm 25 degrees.
\end{abstract}

\subsection{Introduction}

Universal joints are commonly used for applications which require transmission of rotation from one direction to another, or in spatial motion stages such as parallel manipulators [62]. For high precision mechanisms, flexure-based joints are used for their deterministic behavior. However, these joints are restricted to only small deflection angles, suffer from a lack of support stiffness in load carrying directions and support stiffness decreases strongly with deflection [98]. Furthermore, motion in the two independent rotational degrees of freedom is often obtained by serial stacking of compliant elements, resulting in underconstrained intermediate bodies and low support stiffness [15].

Published in: Proceedings of the 32nd annual meeting of the American society for precision engineering (M. Naves, R.G.K.M. Aarts, D.M. Brouwer) 
We have shown that the folded leafspring has proven to be a flexure element that properly maintains a high level of support stiffness over a large range of motion and can be used to construct high stiffness large stroke spherical flexure joints [66]. In this paper, we present a new concept for a two degree of freedom universal flexure joint comprising folded leafsprings, resulting in a high level of stiffness in all support directions over a large range of motion.

\subsection{Conceptual design}

A universal flexure joint is characterized by the property to allow motion in two rotational degrees of freedom while motion in all translational directions and a single rotational direction is constrained (the directions in which loadbearing support is provided). Furthermore, a universal "cardan" joint has the property that both rotation axes intersect to concentrate all rotational motion around a single point, the instant center of rotation $(\boldsymbol{P})$. This freedom space, which contains a collection of all admissible axes of rotation, is schematically illustrated in Fig. 5.1a [37]. The complementary constraint space, which provides the required translational constraints for the given freedom space, is given by all translational constraints intersecting the instant center of rotation combined with additional constraints in the $x y$-plane, illustrated in Fig. 5.1b

In order to obtain this constraint space, flexible members are required which only constrain a single degree of freedom. Wire flexures are suited for this purpose, but do not allow for both a large range of motion and a high support stiffness [92]. Therefore, folded leafsprings are used which also constrain a single translational degree of freedom, which is located along the intersection line of the two leafsprings. Compared to wire flexures, the folded leafsprings typically allow for a larger range of motion with a higher level of support stiffness and load capacity.

To allow for tip-tilt rotational motion only by means of folded leafsprings, the constraints (the folding lines of the folded leafsprings) have to either intersect the instant center of rotation, or have to lay in a plane which coincides with the instant center of rotation, as illustrated in Fig. 5.1b To ensure motion is constrained in all intended four support directions, the folding line of at least one folded leafspring has to intersect the instant center of rotation and at least one has to lay in the plane coinciding with the instant center of rotation. We define the instant center of rotation as the origin (located at $\boldsymbol{P}$ ) and rotation with the rotation axis in the $x y$-plane as the required tip-tilt motion. 


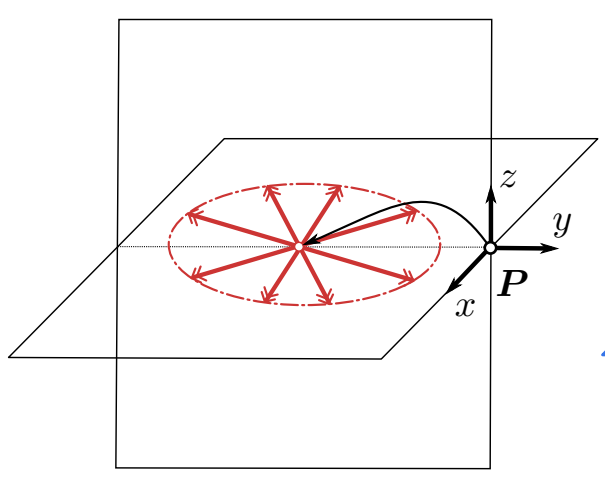

(a)

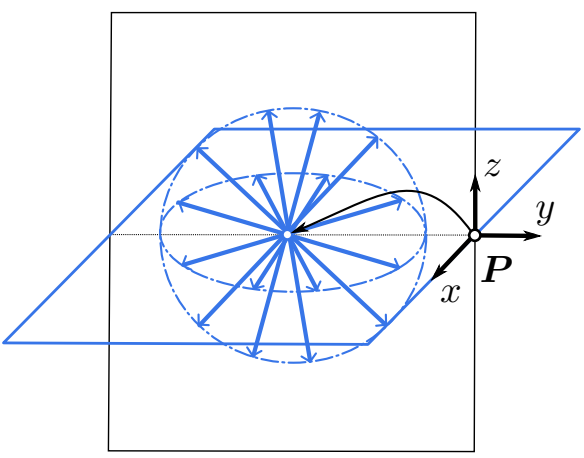

(b)

Figure 5.1: (a) Schematic illustration of the freedom space with all rotational degrees of freedom and (b) the complementary constraint space with all translational constraints for a universal flexure joint with the instant center of rotation indicated by $\boldsymbol{P}$ [37]

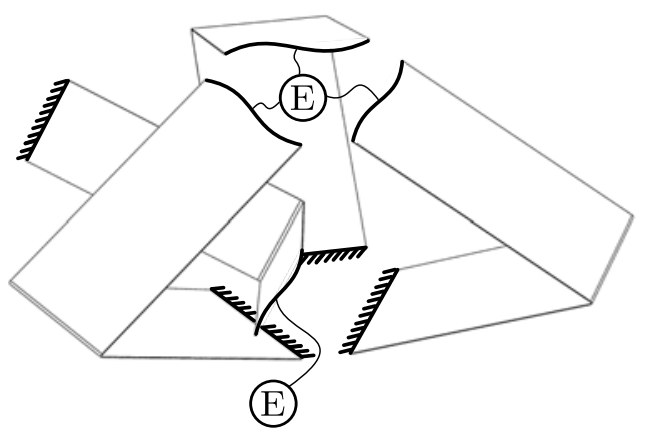

Figure 5.2: Topology with three folded leafsprings with the folding line in the $x y$-plane and a single folded leafspring intersecting the origin $\left(\mathrm{O}^{1} \mathrm{XY}^{3}\right)$

If we only take into account the exact constrained solutions, three distinctive topologies can be conceived. The first solution, shown in Fig. 5.2, is an exact constrained universal joint consisting of three folded leafsprings with the folding line in the $x y$-plane and one folded leafspring intersecting the instant center of rotation (named $\mathrm{O}^{1} \mathrm{XY}^{3}$ ). The second solution consists of two folded leafsprings both intersecting the $x y$-plane and the instant center of rotation, $\mathrm{O}^{2} \mathrm{XY}^{2}$, illustrated in Fig. 5.3. The last remaining solution contains a single folded leafspring in the $x y$-plane and three intersecting the instant center of rotation, $\mathrm{O}^{3} \mathrm{XY}^{1}$, illustrated in Fig. 5.4 


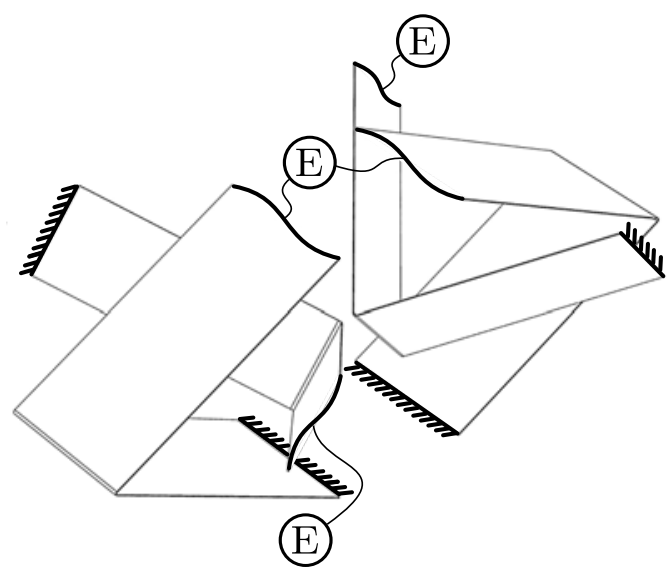

Figure 5.3: Topology with two folded leafsprings with the folding line in the $x y$-plane and two folded leafsprings intersecting the origin $\left(\mathrm{O}^{2} \mathrm{XY} \mathrm{Y}^{2}\right)$

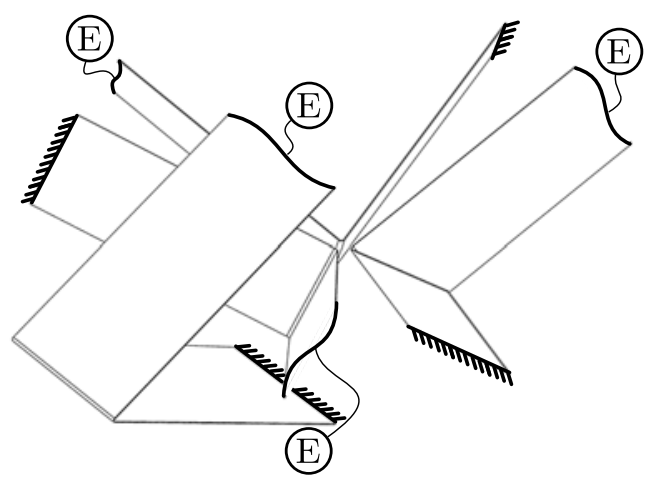

Figure 5.4: Topology with one folded leafspring with the folding line in the $x y$-plane and three folded leafsprings intersecting the origin $\left(\mathrm{O}^{3} \mathrm{XY}^{1}\right)$

To potentially improve support stiffness, overconstrained topologies can be considered by adding extra folded leafsprings. An example of an overconstrained rotational symmetric topology consisting of six folded leafsprings is provided in Fig. 5.5 This topology consists of both three folded leafsprings intersecting the $x y$-plane and the origin $\left(\mathrm{O}^{3} \mathrm{XY}^{3}\right)$. 


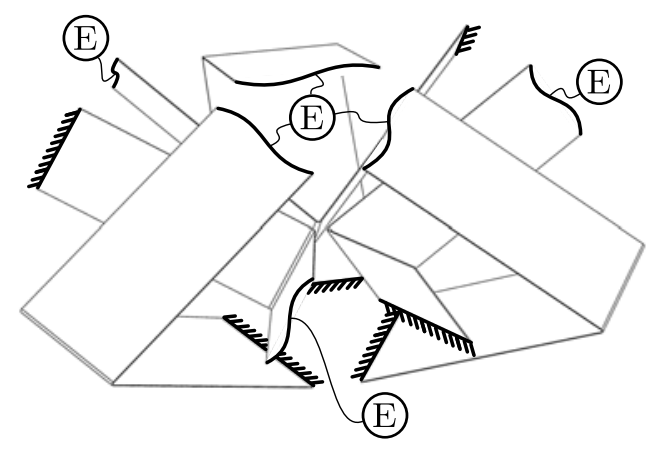

Figure 5.5: Overconstrained rotational symmetric topology with three folded leafsprings with the folding line in the $x y$-plane and three folded leafspring intersecting the origin $\left(\mathrm{O}^{3} \mathrm{XY}^{3}\right)$

\subsection{Performance comparison}

To compare the performance of the described joint topologies, a shape optimization algorithm is used to optimize the designs and to compare the support stiffness [69]. For this comparison the topologies which are not underconstrained, with a maximum of three folded leafsprings with the folding line in either the $x y$-plane or intersecting the origin, are optimized. To restrain the number of design parameters, both the folded leafsprings with the folding line in the $x y$-plane and the folded leafsprings with the folding line intersecting the origin are positioned in a rotational symmetric matter around the $z$-axis. As an example, the joint topologies shown in Fig. 5.2.5.5 are constructed with this rotational-symmetric approach.

For the optimization, the performance is evaluated over the entire range of motion with the flexible multibody software SPACAR [45] to efficiently calculate the strong non-linear stiffness behavior. Due to the intertwined nature of the flexures and to ensure maximum usage of the design space, designs are optimized to only just avoid collision between flexures over its full range of motion. For this optimization case, we consider a universal joint with steel flexures (E-modulus: $210 \mathrm{GPa}$, G-modulus: $80 \mathrm{GPa}$ ) which allows for a tip-tilt bi-directional motion of 25 degrees. Furthermore, maximum actuation moment is restricted to $5 \mathrm{Nm}$ and the stress due to deformation is limited to $300 \mathrm{MPa}$. As optimization criterion, the torsional stiffness around the $z$-axis is considered which is evaluated at maximum tip-tilt angle. Additionally, a constraint is set on the minimum translational support stiffness in $x$-, $y$ - and $z$-direction of $1000 \mathrm{~N} / \mathrm{m}$. The results of the optimized topologies are given in Table 5.1. It has to be noted that the 
Table 5.1: Support stiffness of optimized universal joint topologies at 25 degrees tilt

\begin{tabular}{lllll} 
Topology & $K_{x}(\mathrm{~N} / \mathrm{mm})$ & $K_{y}(\mathrm{~N} / \mathrm{mm})$ & $K_{z}(\mathrm{~N} / \mathrm{mm})$ & $K_{r z}(\mathrm{Nm} / \mathrm{rad})$ \\
\hline \hline $\mathrm{O}^{1} \mathrm{XY}^{3}$ & 13.0 & 14.3 & 1.0 & 28.9 \\
$\mathrm{O}^{2} \mathrm{XY}^{2}$ & 17.7 & 13.4 & 15.9 & 27.6 \\
$\mathrm{O}^{3} \mathrm{XY}^{1}$ & 6.9 & 4.9 & 3.1 & 3.9 \\
$\mathrm{O}^{2} \mathrm{XY}^{3}$ & 1.1 & 2.4 & 3.2 & 21.9 \\
$\mathrm{O}^{3} \mathrm{XY}^{2}$ & 6.4 & 9.1 & 1.7 & 15.7 \\
$\mathrm{O}^{3} \mathrm{XY}^{3}$ & 3.0 & 2.6 & 3.2 & 44.7 \\
\hline
\end{tabular}

optimization results are strongly affected by the considered optimization case and the results are strongly influenced by the chosen boundary conditions and constraints. However, from the obtained results for this specific case, an indication can be given for the level of support stiffness which can be obtained for a universal joint which allows for 25 degrees tip-tilt motion and some distinct properties for some of the joint topologies can be identified.

Table 5.1 shows for the first exact constrained joint topology $\mathrm{O}^{1} \mathrm{XY}^{3}$ a high level of translational support stiffness in $x$ - and $y$-direction combined with a good level of torsional stiffness $\left(K_{r z}\right)$. This high level of stiffness is provided by the three folded leafsprings with the folding-line in the $x y$-plane. Translational stiffness in $z$-direction is limited as it depends on a single flexure intersecting the origin. Joint topology $\mathrm{O}^{2} \mathrm{XY}^{2}$ attains similar stiffness values where torsional and translation support in $x$-direction is provided by the flexures in the $x y$-plane and support in $y$ - and $z$-direction is obtained from the flexures intersecting the origin. At last, joint topology $\mathrm{O}^{3} \mathrm{XY}^{1}$ only provides limited torsional support as only a single flexure is contributing to the torsional stiffness.

Furthermore, it can be observed that the overconstrained asymmetric joint topologies $\left(\mathrm{O}^{2} \mathrm{XY}^{3}\right.$ and $\left.\mathrm{O}^{3} \mathrm{XY}^{2}\right)$ provide no improvement in performance, despite the increased number of flexures. This is caused by a reduction of the feasible design space which is free of collision for the increased number of flexures. Especially for asymmetrical topologies, collision is hard to avoid which effectively results in a reduction of performance. For the symmetric overconstrained topology $\mathrm{O}^{3} \mathrm{XY}^{3}$ collision can be avoided more effectively and therefore, an increase of performance is obtained. 


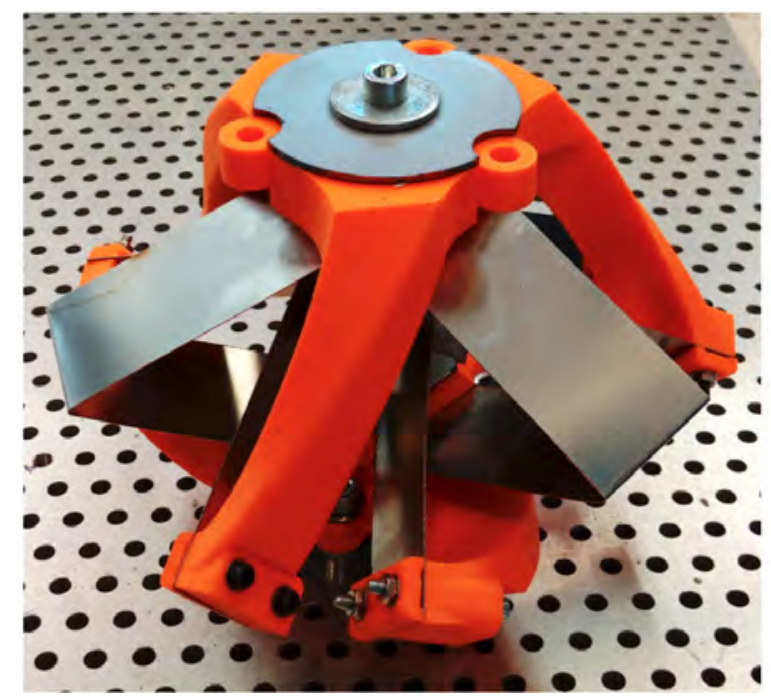

Figure 5.6: Prototype of the $\mathrm{O}^{3} \mathrm{XY}^{3}$-joint consisting of 3D-printed frame parts and spring-steel folded leafsprings

\subsection{Universal joint prototype}

The results obtained from the optimization have been used to design and manufacture the $\mathrm{O}^{3} \mathrm{XY}^{3}$ joint topology. A prototype of this joint is pictured in Fig. 5.6 Due to the spatial layout of the flexures, two 3D-printed frame parts (in orange) are used to connect to both ends of the folded leafsprings. To allow for proper clamping force on the leafsprings, the frame parts are reinforced by two steel plates. Furthermore, the two sets of three folded leafsprings are manufactured from $0.3 \mathrm{~mm}$ thick spring steel and folded to the required folding angle (not necessary 90 degrees). First observations confirm the high level of translational and torsional support stiffness over its full range of motion and the allowed deflection angle of \pm 25 degrees tip-tilt (Fig. 5.7).

Furthermore, for applications of transmitting torque from one direction to another through a variable angle, the relation between input and output angular velocity is of great interest. This behavior is analyzed in more detail in Appendix 5.7.1 


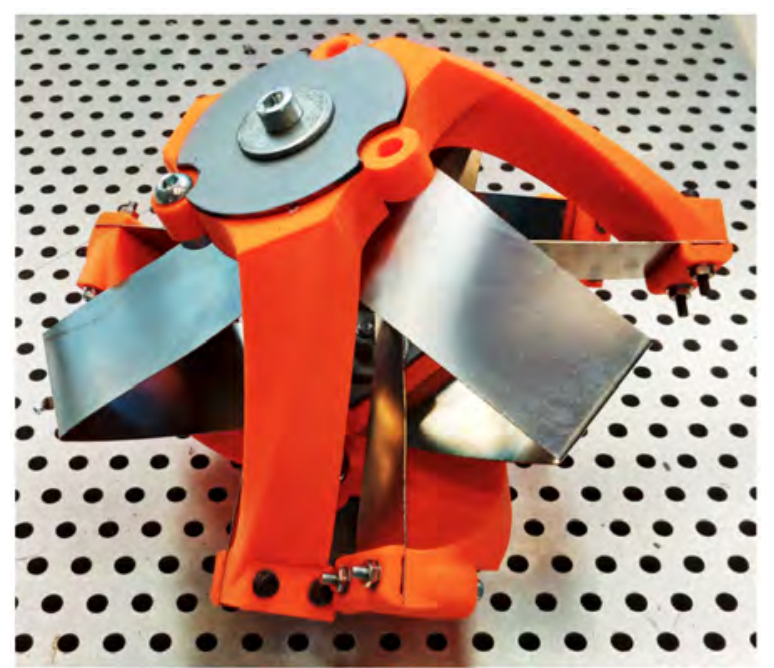

Figure 5.7: Prototype of the $\mathrm{O}^{3} \mathrm{XY}^{3}$-joint at 22.5 degrees tilt angle

\subsection{Discussion}

In order to provide a higher level of support stiffness for the universal joint, overconstrained designs are considered. Although overconstrained designs can result in an increased level of support stiffness, care has to be taken. In order to prevent high levels of internal stress, buckling, or reduction of support stiffness caused by misalignment in overconstrained designs, misalignments have to be reduced to a minimum. For typical flexure mechanisms, misalignments below $50 \%$ of the misalignment at which bifurcation occurs does not significantly affect the systems natural frequencies and stiffness [59]. This gives a good indication of the alignment accuracy which has to be obtained. However, overconstrained issues primarily arise when considering undeflected or small stroke compliant mechanisms, at which the flexures provide a high level of support stiffness in the directions in which motion is constrained. When larger deflections are considered, support stiffness in the constrained directions typically drops. This is caused by the large deformations of the flexures, resulting in a mitigation of internal stress caused by missalignments. Furthermore, the flexures in mechanisms for large stroke applications are often more compliant in the constrained directions due to their different dimensions in order to keep stress below a certain threshold over the full range of motion (e.g. thinner flexures). This generally results in a reduction of overconstraint related problems. 


\subsection{Conclusion}

To obtain a flexure-based universal joint allowing for large tip-tilt deflection while simultaneously maintaining a high level of support stiffness, a series of folded leafsprings are combined and positioned such to allow for tip-tilt motion only. By only considering exact constrained solutions, a good level of support stiffness can be obtained. An increase in performance can be achieved by using overconstrained topologies. However, care has to be taken to maintain a certain symmetry in order to efficiently allow for the avoidance of collision. Based on the obtained optimization results considering a two-degree-of-freedom universal joint allowing for \pm 25 degrees of tip-tilt motion, a prototype has been manufactured providing about $3 \mathrm{~N} / \mathrm{mm}$ stiffness in the translational directions and $45 \mathrm{Nm} / \mathrm{rad}$ in the rotational support direction. 


\subsection{Appendix}

\subsubsection{The $\mathrm{O}^{3} \mathrm{XY}^{3}$ universal flexure joint in torque transmitting applications}

When universal joints are used for the purpose of transmitting rotation (power) from one direction to another through a variable angle, the relation between input and output angular velocity is of great interest. Often, a constant relation between input and output velocity is required in order to prevent vibrations and additional (dynamic) load on the universal joint. Universal joints which feature this constant transfer between input and output velocity is referred to as a Constant-Velocity (CV) joint.

To assess the transfer between input and output velocity, numerical (static) simulations are conducted for which the joint is rotated for a full 360 degrees while evaluating the transfer between input and output rotation, given a certain angle between the input and output axis. Due to the multibody-based formulation of the SPACAR software, the deformations of the elements are invariant to rigid body rotations, allowing for infinite rotations of the joint which enables the evaluation of the transfer between input and output rotations over a full revolution. The ratio between input and output rotational velocity $\dot{\theta}_{\text {out }} / \dot{\theta}_{\text {in }}$ for the universal $\mathrm{O}^{3} \mathrm{XY}^{3}$-joint subjected to an angle of 25 degrees between the input and output axis is provided in Fig. 5.8 For this simulation, the position of the input and output axis are constrained (e.g. input and output axis are constrained by bearings), only allowing for rotational motion of each axis. Results show the output velocity deviates at most by $0.04 \%$ with respect to the input velocity. The magnitude of this deviation scales with the angle between the input and output axis, which is illustrated in Fig. 5.9.

For the case the relative position of the input and output axis are not constrained (e.g. the output axis is not fully constrained by bearings, and the universal joints partially supports the axis), the transfer between input and output rotations change. The transfer between input and output angular velocities for an angle of 25 degrees given the case where only the orientation of the output axis is constrained, is provided at Fig. 5.9 The results show an increased fluctuation in the output velocity of about $0.5 \%$. Differences between both cases are presumably related due to the enforced rotation center of the universal joint for the case of constrained input and output axis, reducing velocity fluctuations.

Note that the transfer between input and output angular velocities described in this appendix are based on static simulations and provide the transfer for 


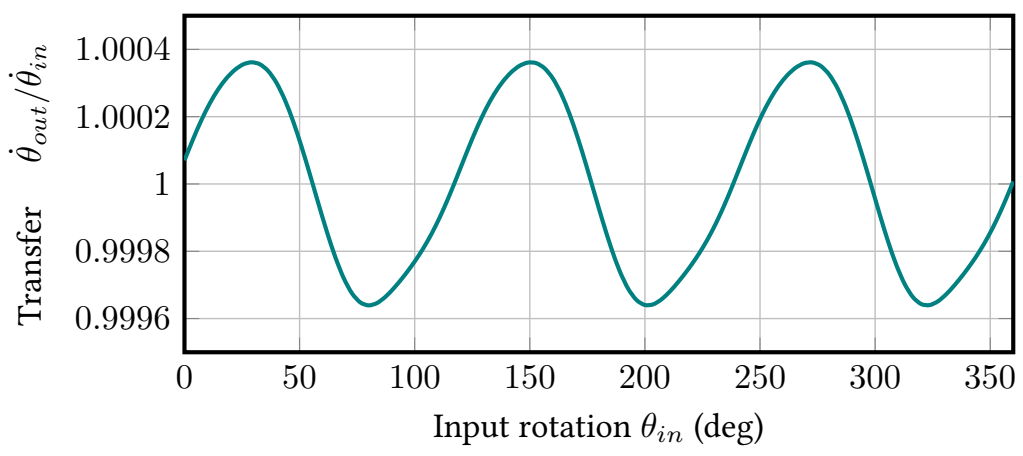

Figure 5.8: Transfer between input and output angular velocity with fixed axes

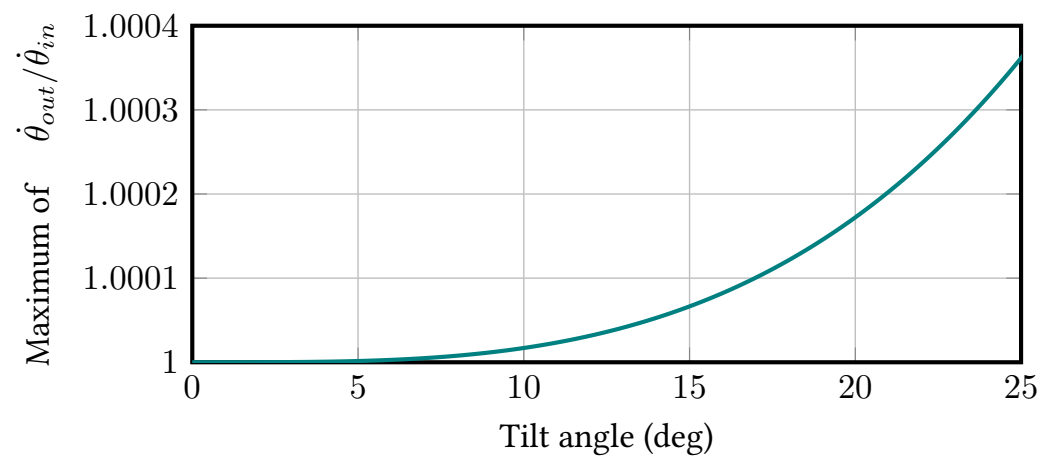

Figure 5.9: Maximum of $\dot{\theta}_{\text {out }} / \dot{\theta}_{\text {in }}$ as function of the angle between the input and output axis

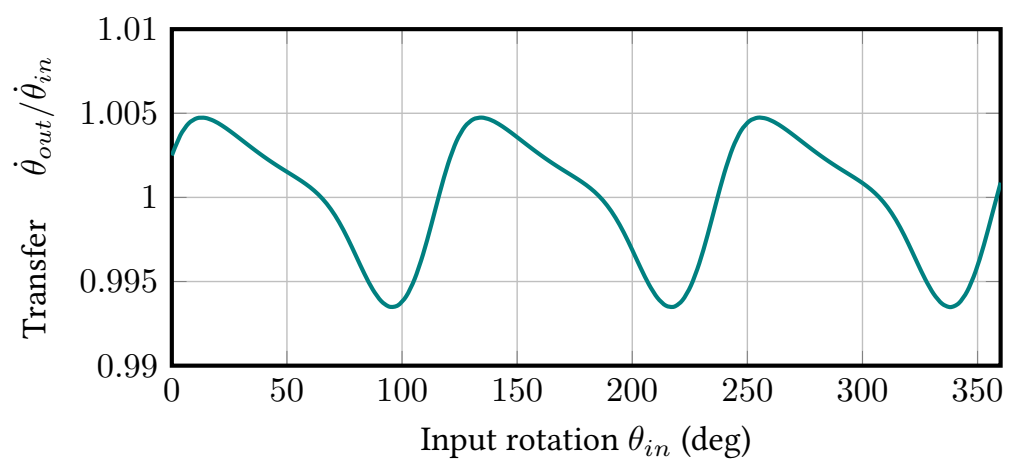

Figure 5.10: Transfer between input and output angular velocity with free axes 
"slow" rotations. Due to internal vibrations, the maximum angular velocity can be limited as high velocities could result in strong (destructive) vibrations. The lowest eigenfrequencies of the presented $\mathrm{O}^{3} \mathrm{XY}^{3}$-joint are around $150 \mathrm{~Hz}$. By considering three periods over a full revolution (Fig. 5.10) resulting from the rotational symmetry of the joints, this limits the maximum rotational velocity $\ll 50 \mathrm{~Hz}(3000 \mathrm{rpm})$ not considering any inertial load attached to the output axis. 


\section{Case study: The T-Flex, a large range of motion flexure-based hexapod}





\title{
Flexure-based 60 degrees stroke actuator suspension for a high torque iron core motor
}

\begin{abstract}
For the actuation of flexure-based precision systems direct drive actuators are used to allow for precise manipulation. For this purpose, often iron-less direct drive actuators are used which do not suffer from parasitic magnetic forces. In contrast, actuators with iron core can provide a higher power density, but require high off-axis stiffness normally too demanding for flexure-based mechanisms, especially when combined with a large range of motion. In this paper, a flexure-based suspension for an iron core direct drive torque motor with a range of motion of 60 degrees ( \pm 30 degrees) and a maximum output torque of $55 \mathrm{Nm}$ is presented. The system provides the required high off-axis stiffness (more than $1000 \mathrm{~N} / \mathrm{mm}$ ) and is able to withstand the high pull-in forces caused by the iron core over the full range of motion. Furthermore, the design is made robust by limiting the deflections in the support directions, effectively rerouting excess forces from the flexures into the frame parts. Experimental validations confirm the high support stiffness and show that the system is able to withstand high actuation and reaction forces. Due to the friction-free mechanical design, only the current noise from the motor driver limits the stand-still performance and the repeatability of the system, resulting in a RMS positioning error of 1.1 urad for an inertia of $0.066 \mathrm{kgm}^{2}$.
\end{abstract}

Published in: Precision Engineering (M. Naves, M. Nijenhuis, W.B.J. Hakvoort, D.M. Brouwer) 


\subsection{Introduction}

Hexapod robots are used for manipulation in up to six independent degrees of freedom [60]. Conventional systems consisting of traditional rolling-element bearings typically allow for a position accuracy down to a micrometer [80, 82, 89]. However, for precision applications such as optical alignment and micro assembly systems an accuracy well below one micrometer is required [5, 17, 34]. This can be achieved by eliminating all sources of play and friction in the joints to improve the determinism of the system [12, 39, 90, 92], which in turn can be obtained by replacing the traditional bearings by flexure-based equivalents [7] 13 88, 110].

To maintain these deterministic properties in actuated robotic systems, the actuators of flexure-based precision systems should also be free of play and friction [97]. To this end, direct drive actuators can be used since they lack tribological contacts. Common Lorentz-type actuators such as voice coil [106], linear ironless [18] or rotary ironless (torque) motors [83] rely on a permanent magnetic field and coil windings (without iron core) to provide actuation forces. Due to the absence of the iron core, high parasitic magnetic forces are avoided [64]. However, the absence of the iron core also results in a reduced strength of the magnetic field and reduced thermal dissipation, which limit the maximum actuation force.

In contrast, Lorentz-type actuators with an iron core, in which the coils are mounted in an iron lamination stack, allow for an increased power density due to the high magnetic flux between the iron core and the permanent magnets [53. [64]. This results in increased actuation forces given a similar size of the actuator. However, the increase in power density comes at the expense of parasitic magnetic forces in the drive direction (cogging) and perpendicular to the drive direction (pull-in). For rotational Lorentz-type actuators, these parasitic pullin forces are proportional to the misalignment between the magnet track and iron core away from the equilibrium position located at the center, resulting in a destabilizing negative stiffness.

The challenge in using a flexure-based suspension is ensuring that the support stiffness and load capacity are sufficiently large to withstand the parasitic magnetic forces and reaction forces from the actuated system. This challenge is compounded for a large range of motion: when deformations increase, support stiffness and load capacity decrease, and the parasitic error motion of flexures increases [8, 73, 112]. Furthermore, flexure hinges suffer from parasitic error 
motions [31 103] that can easily exceed the alignment tolerances of typical actuators resulting in contact between the moving and stationary parts.

In this paper, a flexure-based actuator suspension with 60 degrees range of motion for an iron core actuator is presented. It is designed to meet the requirements for a fully flexure-based large range of motion high precision hexapod, for which high actuation torque is needed. First, in section 6.2 a brief description of the hexapod is provided to derive the requirements for the actuator and the flexurebased bearing construction. Next, in section 6.3 the parasitic motion of three flexure-based cross-hinge candidate designs are compared and analyzed for the considered application. Furthermore, the individual designs of the cross hinges are numerically optimized in section 6.4 in order to obtain the optimal design with respect to support stiffness in the load-bearing directions. In section 6.5 the influence of the pull-in forces on the flexure bearing is analyzed. In section 6.6 a prototype design is presented and experimental validations are provided in section 6.7

\subsection{System description}

In this paper the design of the actuated base joints with a single rotational degree of freedom for a fully flexure-based 6-R̄US hexapod is considered. The hexapod consists of six arms, each made up of one actuated revolute joint at the base and a spherical and universal joint connected to each other via two links. A concept drawing of the hexapod is provided in Fig. 6.1. The hexapod design itself will be described in more detail in Chapter 7 Aim of the system is to pursue high positioning repeatability $<100 \mathrm{~nm}$ combined with a large workspace of $100 \times$ $100 \times 100 \mathrm{~mm}^{3}$ and high accelerations $>5 \mathrm{~g}$.

The design of the flexure-based universal and spherical joints for the hexapod are given by the serial stacked folded leafspring design presented by Naves et al. [68] (Chapter 4). The universal and spherical joints are designed to allow for 25 degrees tip-tilt motion, which describes the rotation angle of the directional axis $\left(\omega_{t}\right.$, schematically illustrated in Fig. 6.2). Rotation around the directional axis for the spherical joint provides pan motion $\left(\omega_{p}\right)$, which is limited to 10 degrees of rotation.

\subsubsection{Requirements}

Given the dimensions of the hexapod, the desired workspace and the range of motion of the spherical joints, a bi-directional range of motion of approximately 


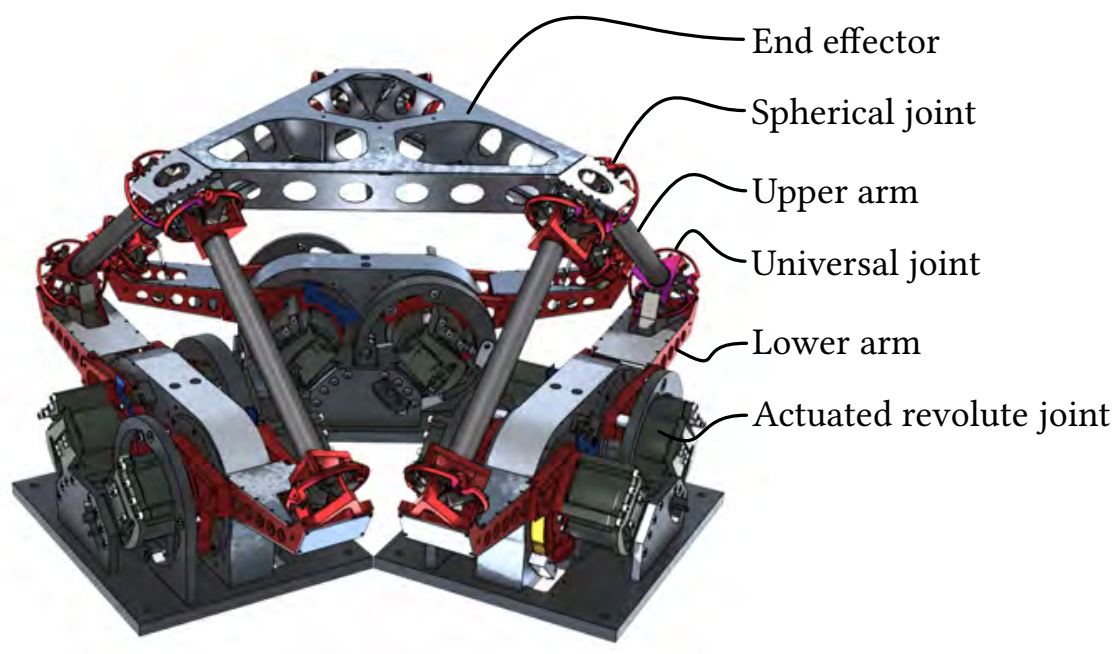

Figure 6.1: Concept design of the 6-奋US parallel manipulator (the T-Flex)

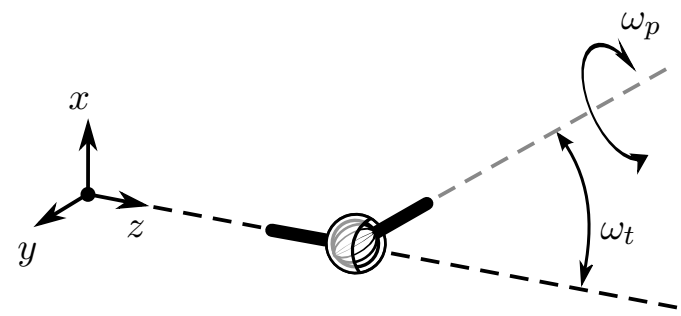

Figure 6.2: Tilt $\left(\omega_{t}\right)$ and pan motion $\left(\omega_{p}\right)$ of a spherical joint

30 degrees is required for the actuated revolute base joints. Additionally, in order to allow for accelerations of the end effector exceeding $5 \mathrm{~g}$, to counteract gravity and to elastically deflect all joints, the required torque is specified at $40 \mathrm{Nm}$. To enable the targeted repeatability, the required rotational repeatability of the actuators is set at $<1 \mu \mathrm{rad}$.

\subsubsection{Actuator selection}

No suitable ironless actuator is found to provide the required torque with an acceptable size or envelope. Therefore, we select an actuator with iron core, and settle for Tecnotion's direct drive iron core torque motor (QTR-A-133-60) with an outer diameter of $133 \mathrm{~mm}$ [94], which allows for a continuous torque of $21.9 \mathrm{Nm}$ 


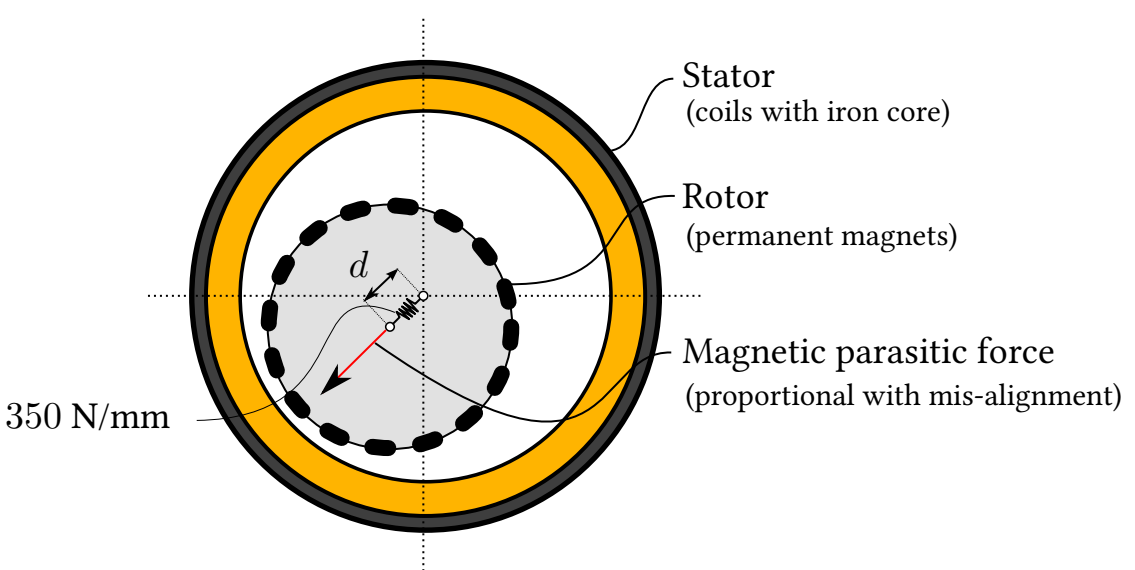

Figure 6.3: Schematic overview of the magnetic (pull-in) forces caused by misalignment $(d)$ between the rotor and stator. Note that the air gap between the rotor and stator is exaggerated.

and an ultimate torque of $55.5 \mathrm{Nm}^{1}$ meeting the torque requirement. Furthermore, due to the moving magnet configuration, the actuator features a stationary coil without cables connected to any moving part, eliminating parasitic forces on moving parts by bending of cables.

An important aspect of an iron core torque motor which has to be taken into account for the design of the flexure-based actuator suspension is the magnetic forces between the iron core and permanent magnets, also known as pull-in forces. As these forces are proportional with the misalignment between the rotor and the stator away from the central equilibrium position, this results in a negative destabilizing stiffness which has to be counteracted by the actuator suspension. A schematic overview of this parasitic force is provided in Fig. 6.3 with $d$ the misalignment between the rotor and stator and the negative stiffness caused by the magnets at $350 \mathrm{~N} / \mathrm{mm}$ and near constant over the range of the air gap $(1 \mathrm{~mm})$ between the rotor and the stator [95].

\subsection{Parasitic motion of revolute flexure joints}

For commonly used flexure joints for a single rotational degree of freedom, such as the cross-spring pivot consisting of two (or more) separate leafsprings [31 39],

1 At ultimate torque the temperature of the coil increases by $20^{\circ} \mathrm{C} / \mathrm{s}$. The maximum duration at which this torque can be maintained depends on thermal properties of the coils housing. 
motion of the shuttle (in this case attached to the rotor) can be approximated by a circular motion around a fixed axis of rotation. However, for larger deflection angles, the motion path of the shuttle starts to diverge from this circular path which deviation can easily exceed the required alignment tolerance of $<1 \mathrm{~mm}$ between the rotor and the stator for the selected actuator. When also taking into account the negative stiffness induced by the permanent magnets and the reaction forces induced by accelerations, additional deformations will cause further deviation from its ideal circular trajectory resulting in additional parasitic motion.

In the next section, the motion characteristics of the cross-spring pivot [31], the cartwheel hinge [77, 90] and the butterfly hinge [33] are compared for the purpose of selecting the optimal joint type. The effect of the negative radial stiffness and reaction forces are disregarded in order to allow for an analytical comparison between the hinge designs. The motion characteristics are compared in terms of their parasitic motion, which is defined as their deviation with respect to the ideal circular motion profile. The properties of the hinge designs including pull-in forces of the rotor is analyzed in more detail in section 6.5 .

\subsubsection{Parasitic motion of the cross-spring pivot}

For the traditional cross-spring pivot [31] consisting of two (or more) individual leafsprings crossing at half their length, the dominant parasitic motion is in $y$ direction and provided by [109]

$$
d_{y}=\frac{L}{12 \cos (\alpha)} \theta^{2}
$$

with $L$ the length of the flexures, $\theta$ the rotation angle of the shuttle and $\alpha$ the relative angle between the leafsprings, schematically illustrated in Fig. 6.4

For a typical value of $\alpha=45$ degrees and a maximum deflection angle of $\theta=$ 30 degrees the resulting parasitic motion is approximately $\frac{L}{31}$. As the maximum allowed parasitic motion is dictated by the design of the actuator, this implies a limit on the allowed length of the flexures to prevent contact between the rotor and stator. Therefore, ensuring a parasitic motion well below $1 \mathrm{~mm}$ requires short flexures. Consequently, the flexures also need to be thin to respect the stress limit for the flexures.

The parasitic motion of the cross-spring pivot can be greatly reduced by choosing $\lambda=1 / 2 \pm \sqrt{5} / 6$, with $\lambda$ providing the relative location at which the flexures cross [103]. For this specific value of $\lambda$, the second order contribution to the 


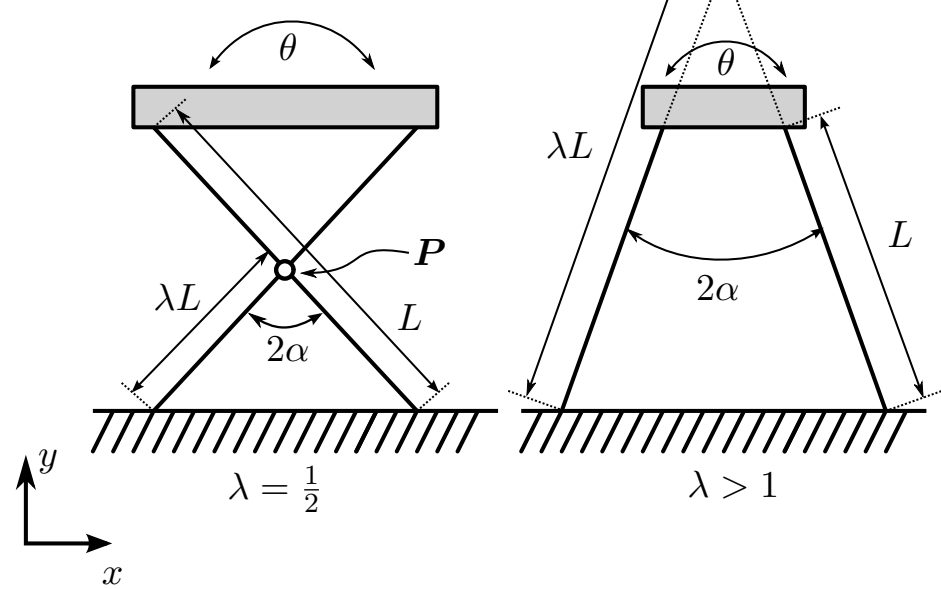

Figure 6.4: Schematic overview of the cross-spring pivot with $\boldsymbol{P}$ indicating the location of the (initial) pivot axis, $\lambda$ the relative location at which the flexures cross and $\theta$ the rotation angle of the shuttle

parasitic motion in $y$-direction is eliminated and the dominant contribution to the parasitic motion is now given by the smaller motion in $x$-direction instead, which is provided by [35]

$$
d_{x}=\frac{\cot ^{2}(\alpha) L}{141 \cos (\alpha)} \theta^{3}
$$

The cross-spring pivot with $\lambda=1 / 2+\sqrt{5} / 6$ is schematically illustrated in Fig. 6.5. For $\alpha=45$ degrees and $\theta=30$ degrees, this results in a parasitic motion of approximately $\frac{L}{695}$, which provides a reduction of more than a factor of 20 compared to the traditional cross-spring pivot with $\lambda=1 / 2$ with the same dimensions. This strongly reduced parasitic motion does allow for longer flexures. However, the asymmetry also results in one flexure part to be short (approximately $0.127 \mathrm{~L}$ ), causing the bending stress due to deflection to be high, which in turn requires the thickness of the flexures to be small.

Furthermore, it has to be noted that the magnitude of the parasitic motion for this hinge design is more sensitive to deviations in the value of $\lambda$. For example, the shift in $y$-direction is provided by

$$
d_{y}=-70 \frac{9 \lambda^{2}-9 \lambda+1}{1050 \cos (\alpha)} L \theta^{2}
$$




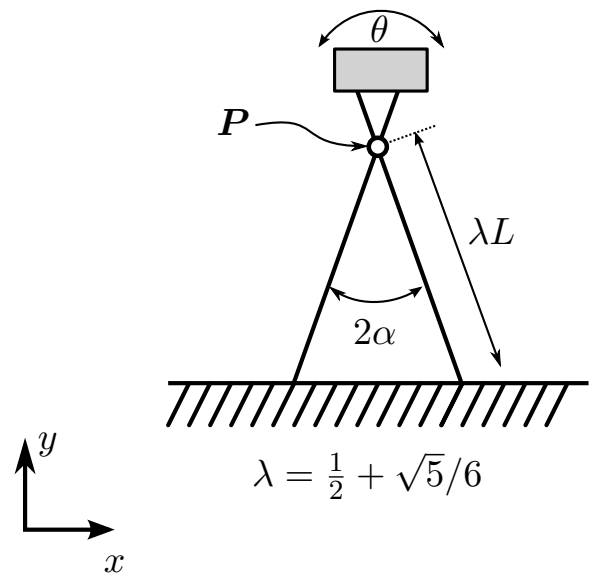

Figure 6.5: Schematic overview of a cross-spring pivot with $\lambda=1 / 2+\sqrt{5} / 6$ resulting in minimal parasitic motion [103]

for which the derivative with respect to $\lambda$ is given by

$$
\frac{\Delta d_{y}}{\Delta \lambda}=-70 \frac{18 \lambda-9}{1050 \cos (\alpha)} L \theta^{2}
$$

At $\lambda=1 / 2+\sqrt{5} / 6$ considering $\alpha=45$ degrees and $\theta=30$ degrees, this results in a sensitivity of

$$
\Delta d_{y} \approx-\frac{1}{6} L \Delta \lambda
$$

which is significant with respect to the obtained parasitic motion from Eq. 6.2 for already small values of $\Delta \lambda$. Therefore, this hinge design would require narrow alignment and manufacturing tolerances in order to actually realize this small magnitude for the parasitic motion.

\subsubsection{The cartwheel hinge}

An alternative flexure-based design for a single rotational degree of freedom is provided by the cartwheel hinge [77, 90], schematically illustrated in Fig. 6.6 which consists of two leafsprings intersecting at half their length. To evaluate the characteristics of the cartwheel hinge, the results from the previous section can be used by considering the cartwheel hinge as a stacked arrangement of two cross-spring pivots with $\lambda=1$ and $\lambda=0$. To obtain a similar footprint for the cross-spring pivot and the cartwheel hinge for a specific value of $L$, the flexure 

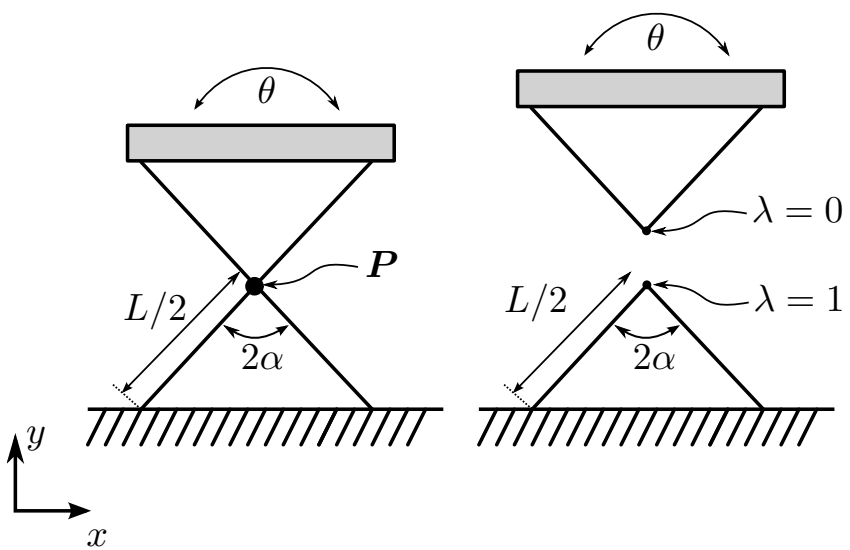

Figure 6.6: Schematic overview of the cartwheel hinge with $\mathrm{P}$ indicating the location of the pivot axis and $\theta$ the rotation angle of the shuttle. Note that for the cartwheel hinge the flexures are interconnected at point $\boldsymbol{P}$, in contrast with the cross-spring pivot.

length of each flexure in the cartwheel hinge is given length $L / 2$, schematically illustrated in Fig. 6.6. The resulting parasitic motion is then given by [87]

$$
d_{y}=\frac{L}{63 \cos (\alpha)} \theta^{2}
$$

This effectively results in a parasitic motion of $\frac{L}{162}$ for $\alpha=45$ degrees and $\theta=30$ degrees. Compared to the cross-spring pivot, this hinge suffers from an intermediate level of parasitic motion, in-between the standard cross-spring pivot with $\lambda=1 / 2$ and the cross-spring pivot with minimal parasitic motion given $\lambda=1 / 2 \pm \sqrt{5} / 6$.

\subsubsection{The butterfly hinge}

A third design for a cross-hinge, which is known for its small parasitic motion due to the smart compounding of leafsprings, is provided by the butterfly hinge design [33]. This hinge effectively consists of a stacked arrangement of four cross-spring pivots with coinciding rotation axes, schematically illustrated in Fig. 6.7 For this joint, parasitic motion of the first and fourth cross-spring pivot in the stacked arrangement is compensated for by opposed parasitic motion of the second and third cross-spring pivot. Therefore, parasitic motion is reduced resulting in [35]

$$
d_{y}=\left(\frac{1}{\cos \left(\alpha_{2}\right)}-\frac{1}{\cos \left(\alpha_{1}\right)}\right) \frac{9 \lambda^{2}-9 \lambda+1}{240} L \theta^{2}
$$



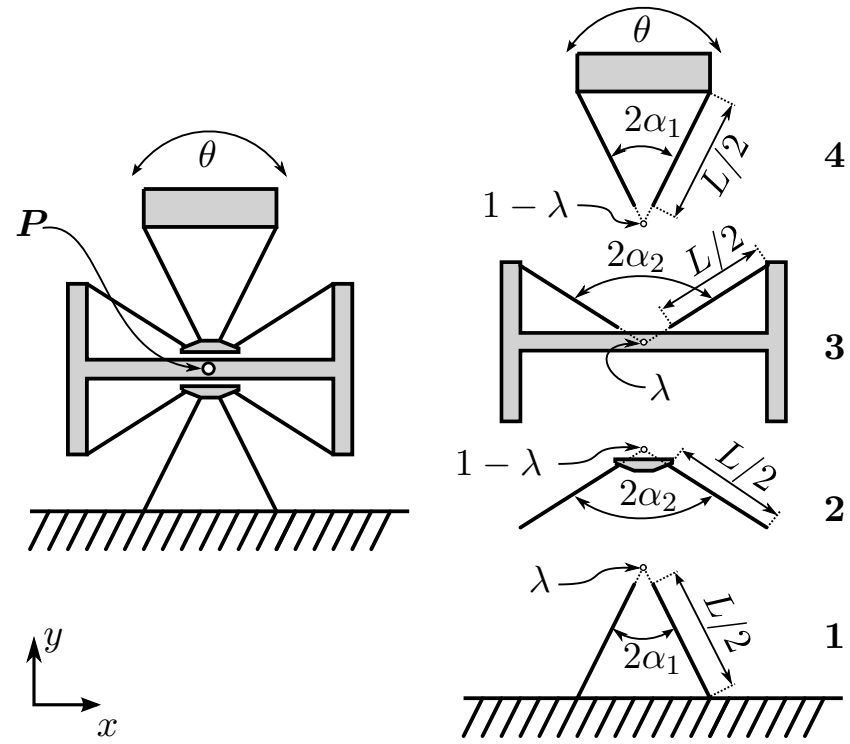

Figure 6.7: Schematic overview of the butterfly hinge with $\boldsymbol{P}$ indicating the location of the (initial) pivot axis, $\lambda$ the relative location at which the flexures cross and $\theta$ the rotation angle of the shuttle

with $\lambda>1$ in order to prevent interference between the individual cross-spring pivots in the stacked arrangement and with $\alpha_{1}$ and $\alpha_{2}$ providing the angle between the inner and outer set of flexures as indicated in Fig. 6.7. Considering a realistic value of $\lambda=1.1$ combined with feasible values of $\alpha_{1}$ and $\alpha_{2}$ which allow for 30 degrees deflection without interference between the flexures, for example $\alpha_{1}=35$ degrees and $\alpha_{2}=55$ degrees, the resulting parasitic motion is $\frac{L}{682}$.

\subsection{Numerical optimization}

In order to select the most suitable hinge topology for the bearing suspension, the design of the cross-spring pivot, cartwheel hinge and butterfly hinge are optimized numerically.

\subsubsection{Optimization procedure}

For the optimization procedure, an adapted Nelder-Mead based shape optimization algorithm [69] is used to optimize the length $(L)$ and thickness $(t)$ of the 
flexures to obtain maximum radial (off-axis) support stiffness. To obtain similar stiffness values in all radial directions, a value of $\alpha=45$ degrees is selected for the cross-spring pivot and the cartwheel hinge ( $\alpha=45$ degrees can be considered optimal when maximizing the critical off-axis stiffness). For the butterfly hinge, $\alpha_{1}=35$ degrees and $\alpha_{2}=55$ degrees is selected, which is chosen as close to 45 degrees as possible while still allowing for 30 degrees deflection in each direction.

Furthermore, the minimum allowed flexure thickness is set at $0.2 \mathrm{~mm}$, which is considered the minimum flexure thickness which can be manufactured (with sufficient accuracy) by wire EDM, which is the selected manufacturing method for the flexures. The length of the flexures $(L)$ is limited according to the provided equations in section 6.3 in order to assure parasitic motion below $1 \mathrm{~mm}$. Note that for the actual system a parasitic motion well below $1 \mathrm{~mm}$ is required in order to allow for additional deflection due to the reaction forces involved. However, for an initial comparison between the joints, we accept a parasitic motion up to $1 \mathrm{~mm}$ which provides maximum "design" freedom in the optimization procedure, resulting in the "best-case" off-axis support stiffness that can be obtained with each of the flexure joints, which will be used for comparison.

For the optimization procedure, the performance is evaluated over the entire range of motion with the flexible multibody software SPACAR [45] which uses a series of interconnected nonlinear 3D finite beam elements which include the geometric nonlinearities to capture the relevant behavior of the flexures. The objective of the optimization is to maximize the radial support stiffness in the most compliant direction $\left(K_{\text {crit }}\right)$ over the range of motion of 60 degrees (-30 degrees to +30 degrees) with the goal to obtain sufficient resistance to pull-in and reaction forces. As material, tooling steel (Stavax) is selected where we limit the allowable stress to $600 \mathrm{MPa}$, which is about $40 \%$ of the yield stress of the material. Furthermore, the maximum width of the hinges is limited to $100 \mathrm{~mm}$ measured in the direction of the rotation axis.

\subsubsection{Optimization results}

An overview of the optimization results is presented in Table 6.1 with each design just within the allowed stress limit of $600 \mathrm{MPa}$ at their maximum deflection angle.

From the results it can be confirmed that the cross-spring pivot with $\lambda=0.5$ requires short flexures in order to ensure parasitic motion below $1 \mathrm{~mm}$, limiting the flexure length to $31 \mathrm{~mm}$. Furthermore, this cross-spring pivot provides the 


\begin{tabular}{llllll} 
& & CP & CP & CFH & BFH \\
\hline \hline$\lambda$ & & 0.5 & $\frac{1}{2} \pm \frac{\sqrt{5}}{6}$ & - & 1.1 \\
$L$ & $(\mathrm{~mm})$ & 31 & 63 & 153 & 70 \\
$t$ & $(\mathrm{~mm})$ & 0.27 & 0.20 & 0.42 & 0.33 \\
$\alpha_{(1)}$ & $(\mathrm{deg})$ & 45 & 45 & 45 & 35 \\
$\alpha_{2}$ & $(\mathrm{deg})$ & - & - & - & 55 \\
\hline$K_{\text {crit }}$ & $(\mathrm{N} / \mathrm{mm})$ & 1492 & 64 & 869 & 1283 \\
$d$ & $(\mathrm{~mm})$ & 0.98 & 0.09 & 0.94 & 0.09 \\
\hline
\end{tabular}

Table 6.1: Optimization results with the radial support stiffness $\left(K_{\text {crit }}\right)$ and parasitic motion $(d)$ provided at the maximum deflection angle of $\theta=30$ degrees. (CP: Crossspring Pivot; $\mathrm{CFH}$ : Cartwheel Flexure Hinge; BFH: Butterfly Hinge)

highest level of support stiffness at the maximum deflection angle of $1492 \mathrm{~N} / \mathrm{mm}$. In contrast, the cross-spring pivot with $\lambda=1 / 2 \pm \sqrt{5} / 6$ features a much smaller parasitic motion of only $0.09 \mathrm{~mm}$, although it comes at the expense of a strongly reduced support stiffness $(64 \mathrm{~N} / \mathrm{mm})$. Taking into account the negative stiffness induced by the rotor $(-350 \mathrm{~N} / \mathrm{mm})$, the obtained support stiffness is insufficient. The relatively low support stiffness can be related to its asymmetric design, resulting in high stress concentrations that require long and slender flexures to keep stress below $600 \mathrm{MPa}$.

The parasitic motion of the cartwheel flexure hinge is comparable with the crossspring pivot with $\lambda=1 / 2$ while providing slightly less support stiffness $(869 \mathrm{~N} / \mathrm{mm})$. At last, the butterfly hinge features a small magnitude for the parasitic motion combined with a relatively high support stiffness of $1283 \mathrm{~N} / \mathrm{mm}$.

\subsection{Influence of negative rotor stiffness}

The numerically evaluated values for stiffness and parasitic motion discussed in the previous section do not take into account the additional pull-in forces due to parasitic motion of the rotor caused by the magnets. These magnetic forces are not included in the optimization procedure as it could result in buckling of the flexures during the static analysis of the flexure joints, which would result in problems with respect to convergence in numerical simulations due to its strongly non-linear behavior and possibly absence of a static equilibrium. Consequently, this lack of convergence would result in a great increase in the required computational effort for the optimization of the flexure joints. 
To overcome this, the influence of these forces are excluded in the optimization procedure and analyzed separately in this section. The cross-spring pivot with $\lambda=1 / 2 \pm \sqrt{5} / 6$ is not considered as the inherent support stiffness of this joint $(64 \mathrm{~N} / \mathrm{mm}$ at the maximum deflection angle) is already insufficient to counteract for the negative stiffness induced by the magnetic forces $(-350 \mathrm{~N} / \mathrm{mm})$.

To model the influence of the magnetic forces, a spring with negative stiffness is modeled between the shuttle of each flexure joint (the rotor) and the fixed world which captures the static load caused by the combination of negative stiffness and parasitic motion of the rotor. The spring is located at the initial location of the rotation axis of the flexure hinge, considering perfect initial alignment between the rotor and the stator (meaning zero reaction forces at $\theta=0$ degrees).

\subsubsection{Simulation results including negative rotor stiffness}

The support stiffness of the optimized flexure joints without negative rotor stiffness and the support stiffness with negative rotor stiffness and resulting reaction forces are provided in Fig. $6.8 \mathrm{a}$ and fig. $6.8 \mathrm{~b}$ respectively. The maximum Von Mises stress in the flexures (including the negative rotor stiffness and the resulting reaction forces) is provided in Fig. 6.9

From the results it can be observed that the reaction forces caused by the negative stiffness have a large influence in the observed off-axis stiffness and maximum stress in the flexures, especially when considering larger deflection angles. For the cartwheel flexure hinge, the reaction forces caused by parasitic motion (which is in the negative $y$-direction) result in large compressive loads in the flexures causing load softening [4, 73]. Due to these forces, the flexures start to buckle at a deflection angle of $\theta \approx 25$ degrees at which the off-axis support stiffness deteriorates rapidly, resulting in insufficient stiffness to counteract the magnetic forces. Furthermore, the reaction forces result in a slight decrease in maximum stress due to the changed stress-distribution in the flexures. More specifically, the maximum stress in a cartwheel hinge without load in the loadbearing directions occurs at the intersection of the individual flexures (at the center of the joint). However, when the flexures are subjected to compressive loads, stress in the center of the joint decreases resulting in a reduced stress maximum, although stress increases at other locations in the flexures.

In contrast to the cartwheel hinge, parasitic motion of the cross-spring pivot is oriented in the positive $y$-direction, resulting in large tensile loads in the flexures. Due to the tensile loads, stress stiffening is observed resulting in an increase in off-axis support stiffness for the cross-spring pivot. Despite the increase in 


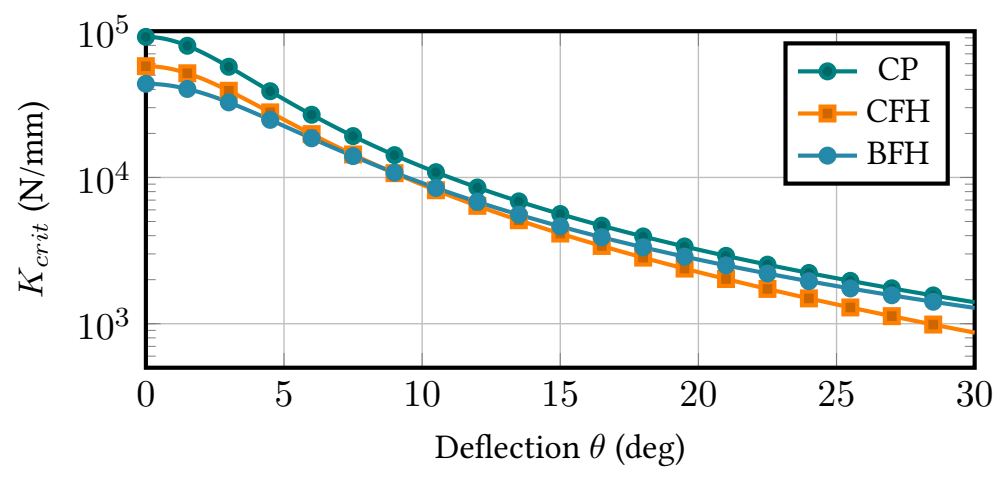

(a)

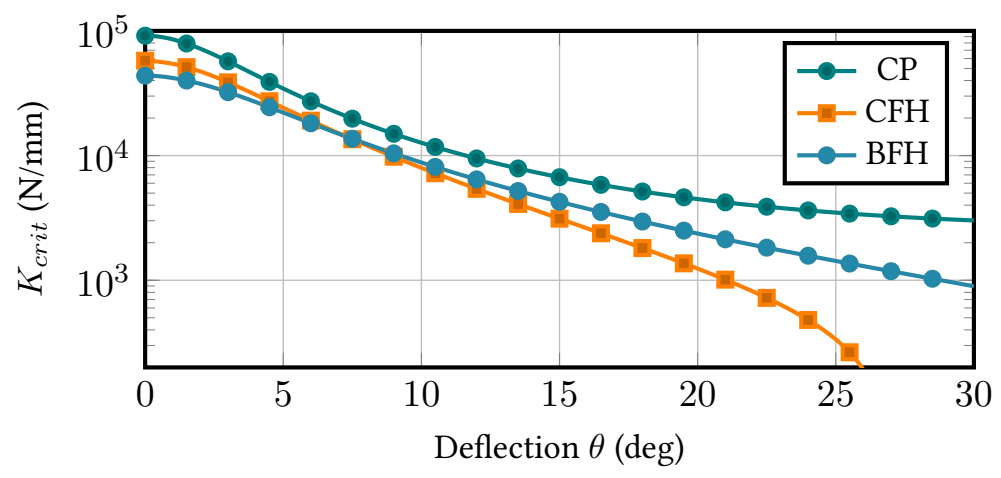

(b)

Figure 6.8: Support stiffness of optimized flexure joints (a) without negative rotor stiffness and (b) with negative rotor stiffness and reaction forces caused by parasitic motion.

CP: Cross-spring Pivot, CFH: Cartwheel Flexure Hinge, BFH: ButterFly Hinge

support stiffness in the cross-spring pivot, the high tensile load also results in an increased curvature of the flexures near the clamped ends. Therefore, stress is strongly increased (Fig. 6.9) up to almost $1200 \mathrm{MPa}$ at the maximum deflection angle, which is an increase of about a factor of two.

For the butterfly hinge, support stiffness and the maximum stress in the flexures remains mostly unaffected by the additional reaction forces. Due to the small magnitude of the parasitic motion, the reaction forces are relatively small. Therefore, no excessive tensile or compressive loads occur in the flexures which would otherwise jeopardize its performance.

Overall, it can be concluded that the butterfly hinge is most suited for the rotor 


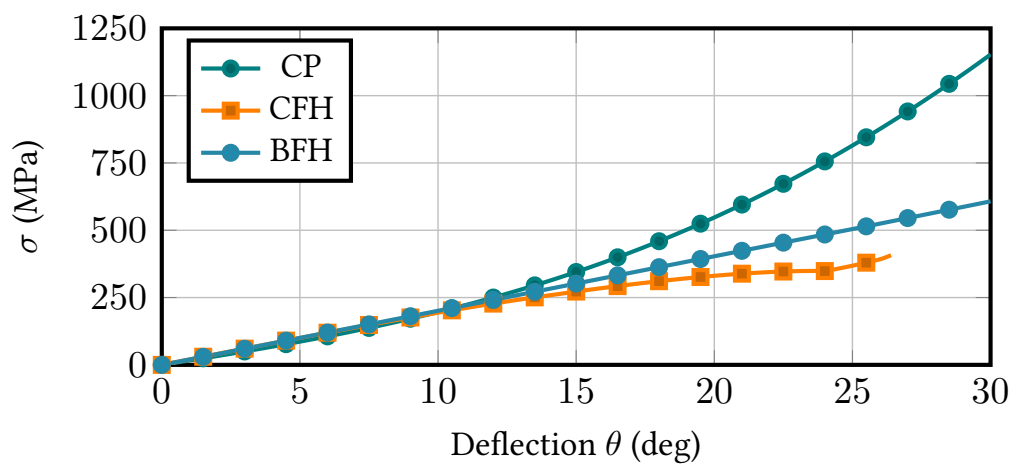

Figure 6.9: Maximum stress (Von Mises) of optimized flexure joints with negative rotor stiffness and reaction forces caused by parasitic motion included.

CP: Cross-spring Pivot, CFH: Cartwheel Flexure Hinge, BFH: ButterFly Hinge

suspension, featuring low parasitic motion and high support stiffness in the offaxis directions. Furthermore, it is able to withstand the reaction forces induced by the rotor without suffering from a strong increase of stress in the flexures or a decrease of support stiffness due to compressive forces.

\subsubsection{Load capacity}

To further assess the performance of the butterfly hinge, the maximum load capacity of this joint is evaluated. This ultimate load capacity is defined as the load at which the parasitic deflection exceeds $1 \mathrm{~mm}$ (at the maximum deflection angle of 30 degrees), which results in contact between the rotor and the stator.

The maximum load capacity of the butterfly hinge is determined iteratively by gradually increasing load in the critical off-axis direction. Due to the magnetic pull-in forces in the direction of the parasitic motion, the off-axis stiffness in this direction is strongly reduced. Therefore, the critical most compliant off-axis direction is given by the direction of parasitic motion, which is in the vertical $y$-direction given by Eq. 6.7). Simulation results including this additional load is provided in Fig. 6.10, which take into account the negative rotor stiffness and contact between the rotor and stator. This simulation is conducted at a deflection angle of 30 degrees, providing the magnitude of the parasitic motion (line with circular markers) and the support stiffness (line with rectangular markers) as a function of the applied load. 


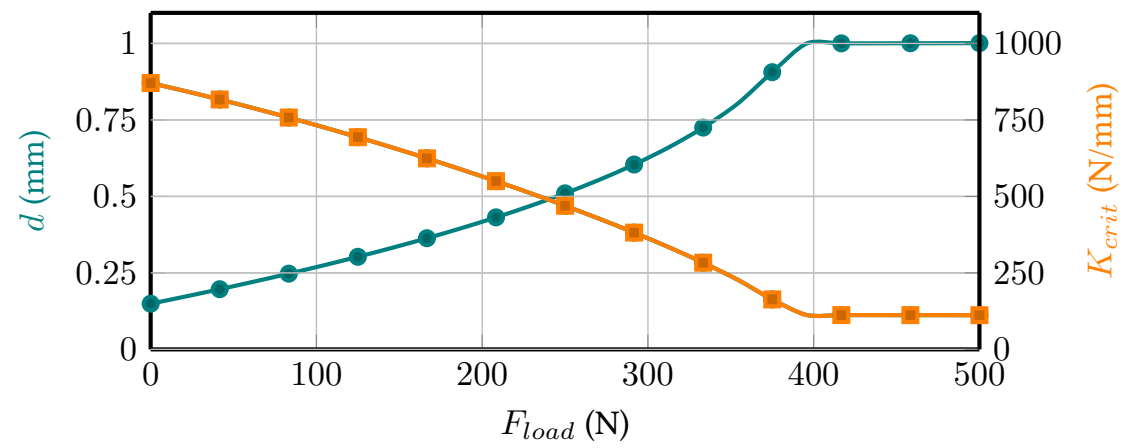

Figure 6.10: Parasitic motion (circles) and critical off-axis support stiffness (rectangles) of the butterfly hinge for increasing payload $\left(F_{\text {load }}\right)$ at the maximum deflection angle of $\theta=30^{\circ}$

From these results it can be concluded that the support stiffness decreases with increasing load, resulting in a support stiffness of about $100 \mathrm{~N} / \mathrm{mm}$ at the maximum payload of $400 \mathrm{~N}$ (including the negative stiffness of $-350 \mathrm{~N} / \mathrm{mm}$ ), resulting in contact between the rotor and the stator. It has to be noted that the stress in the flexures increases only slightly over a rather large displacement range of the rotor due to the decrease in support stiffness (the flexures are close to buckling), resulting in an increase of only $200 \mathrm{MPa}$ when contact between the stator and rotor occurs. Therefore, excessive loads are rerouted through the frame parts without excessive stress levels exceeding the yield stress. This relieves the flexures and makes the system robust to extreme loading conditions. The $400 \mathrm{~N}$ of maximum load capacity provides a worst-case capacity at the maximum deflection angle. For smaller deflection angles, a load $>400 \mathrm{~N}$ is allowed.

\subsection{Detailed design}

Based on the optimization results, a prototype of the flexible actuator suspension for the selected torque motor is constructed by using the butterfly hinge design with the design parameters according to Table 6.1 First, the detailed design of the butterfly hinge is discussed, followed by a detailed design of the actuator suspension. 
(2)

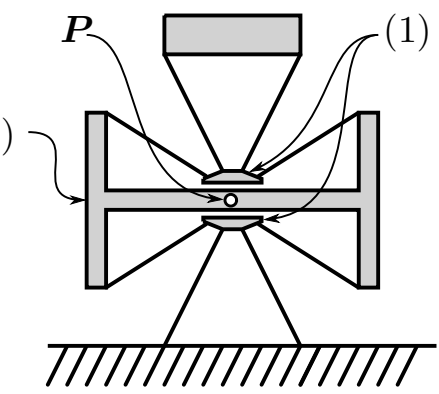

(a)

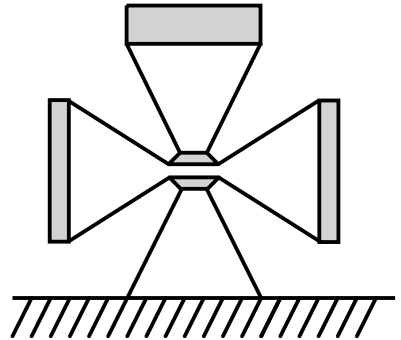

(b)

Figure 6.11: (a) Schematic overview of a butterfly hinge with: (1) small intermediate body with high natural frequency (2) large intermediate body with low natural frequency (b) Schematic overview of butterfly hinge with seperated intermediate body

\subsubsection{Butterfly hinge design}

The butterfly hinge as presented in section 6.3.3 contains three intermediate (underconstrained) bodies. For most flexure-based mechanisms, underconstrained intermediate bodies dramatically decrease support stiffness due to coupling between external loads on the end effector and loads in the underconstrained degrees of freedom. Especially for large deflection angles, this results in high compliance [48] in the load carrying directions. However, for the butterfly hinge, the instant center of rotation of each intermediate body and the end effector coincide and do not vary much over the range of motion, and therefore external loads on the end effector result in only limited reaction moments in the degrees of freedom of the intermediate bodies. As a consequence, the support stiffness is not deteriorated by the underconstraints. However, with respect to the dynamic behavior of the joint, the underconstrained intermediate bodies can result in (low frequency) unwanted vibrations in the actuator suspension.

The two small intermediate bodies (1) positioned between the first and second flexure set and the third and fourth flexure set, schematically illustrated in Fig. 6.11a have a high natural frequency in the underconstrained degree of freedom due to the low inertia with respect to the rotation axis of the joint $(\boldsymbol{P})$. Therefore, these intermediate bodies do not impose unwanted vibrations in the frequency range of interest (i.e. 0-100 Hz). However, the large intermediate body (2) positioned between the second and third flexure set does potentially lead to unwanted vibrations with low natural frequency due to its larger inertia. In order to eliminate this underconstrained, an additional slaving mechanism is required which couples motion of the end effector (rotor) and this intermediate body at 


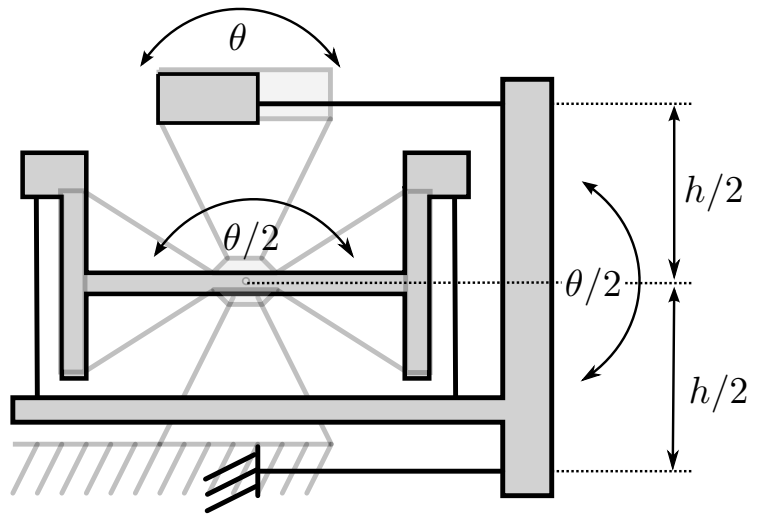

Figure 6.12: Schematic overview of butterfly hinge with slaving mechanism.

a two-to-one ratio [32, 38], eliminating the redundant degree of freedom. A schematic overview of the butterfly hinge with a slaving mechanism is provided in Fig. 6.12

In addition to the slaving mechanism, parasitic motion of the butterfly hinge and the mass of the two small intermediate bodies is reduced to a minimum (increasing its natural frequency) by splitting the large intermediate body between the second and third flexure set in half, schematically illustrated in Fig. 6.11b. Hence, all flexures can be interconnected more closely to the instant center of rotation resulting in a reduction of $\lambda$, which provides reduced parasitic motion, increased support stiffness and reduced stress levels. For the final butterfly hinge design, this modification allows for $\lambda \approx 1.1$ with only a gap of $1.5 \mathrm{~mm}$ between the two small intermediate bodies (a smaller gap could not be achieved due to the minimum clearance required for manufacturing). To connect the two separate bodies between the second and third flexure set, the bodies are rigidly connected by means of the central frame part of the slaving mechanism, which is placed on top (in a different plane) of the butterfly hinge (see also Fig. 6.12).

\subsubsection{Actuator suspension design}

A photograph and a design drawing with exploded view of the designed prototype is provided in Figs. 6.13 and 6.14 which shows the suspension for two actuators with parallel rotation axes (rotor and flexure bearing only present for the actuator on the left-hand side). In the design, a butterfly hinge (1) with a width of $50 \mathrm{~mm}$ and slaving mechanism (2) are placed on each side of an aluminum rotor hub (3) which carries the permanent magnets of the rotor (4). By 


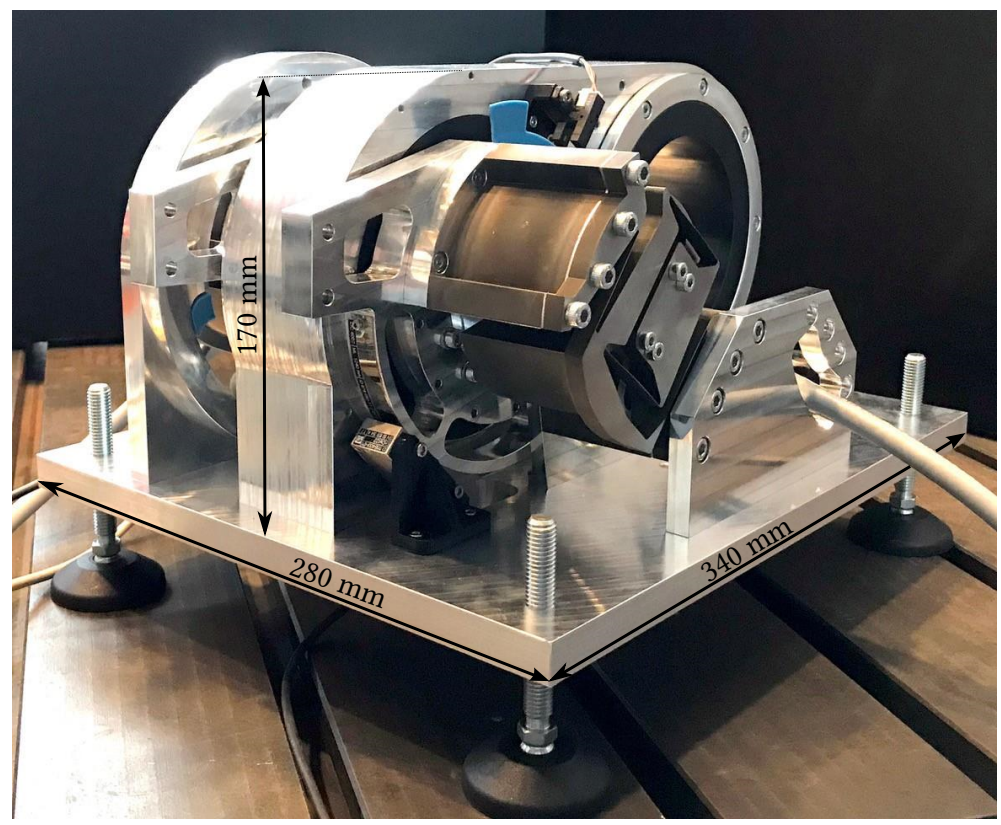

Figure 6.13: Photograph with the main dimensions of the flexure-based actuator suspension

placing two butterfly hinges at each side of the rotor, high stiffness in both the translational and rotational load carrying directions are provided. The central "large" intermediate bodies which are constrained by the slaving mechanisms are interconnected with an aluminum body (5). This body is placed inside the rotor hub and provides a stiff connection between the intermediate slaved bodies. Furthermore, the rotation of the rotor is measured by a linear optical encoder (6) (Heidenhain's LIC 4119 with a $1 \mathrm{~nm}$ measuring step), placed at a radius of $75 \mathrm{~mm}$ (resulting in a resolution of 13 nrad) which is able to maintain focus over the entire range of motion taking into account the parasitic motion of the rotor.

In order to prevent excessive rotations, a mechanical stop (7) and optical switch (8) near the end of the range of motion are added to the rotor. The actuator's stator (9), which is the stationary part of the actuator containing the coils, is fixed inside an aluminum frame (10). This frame holds the stator and improves the thermal dissipation for the current conducting coil. Furthermore, the housing is equipped with cooling channels (11) to provide active cooling to the stator for high load applications.

As two butterfly hinges at each side of the rotor are used for supporting the actuator, the system is overconstrained which could result in excessive stress, 


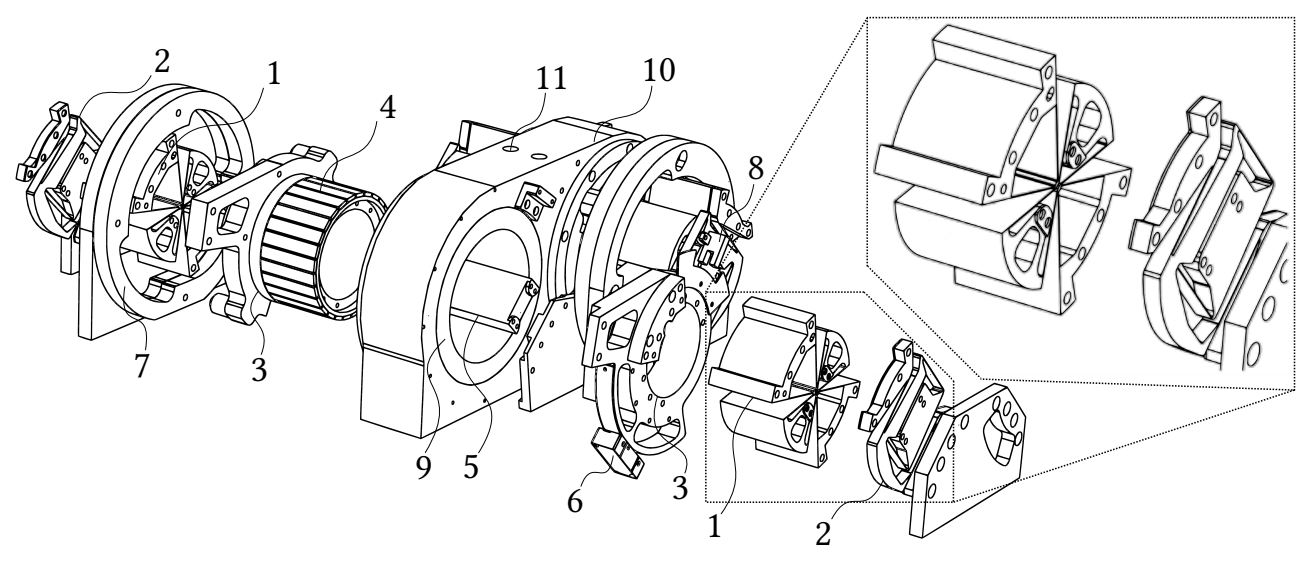

Figure 6.14: Exploded view of actuator suspension. 1) Butterfly hinge 2) Slaving mechanism 3) Rotor-hub 4) Permanent magnets of the rotor 5) Body coupling intermediate bodies of both butterfly hinges 6) Encoder 7) Mechanical stop 8) Optical switch 9) Actuator's stator 10) Stator housing 11) Cooling channels.

increased stiffness in the degree of freedom or even decreased stiffness in the support directions. Therefore, care is taken for the positioning of the two individual butterfly hinges ensuring good alignment between them. In particular the location of the rotation axis of both butterfly hinges requires proper alignment, which is ensured by using dowel pins for both hinges.

Furthermore, it has to be noted that insertion of the rotor during assembly requires special attention in order to provide resistance to the negative radial stiffness and to ensure good alignment between the stator and rotor. For this purpose, a linear guidance and a spacer for filling the air gap between the rotor and stator is used, which is removed after assembly.

\subsubsection{Controller design}

The absence of static friction allows accurate positioning, because no limit cycling will occur when using integral control action. On the other hand, the absence of self-locking friction makes the rotor position sensitive to disturbances and the combination with the rotational stiffness requires the controller to be active even after the target position is reached. The main source of disturbance is found to be the noise on the current introduced by the motor driver used to power the actuator, which is approximately white noise. Therefore, the transfer from the current noise to the position error (the input sensitivity) is minimized 
by $\mathrm{H}_{2}$ feedback control design [52]. The mechanics model for the control design is obtained from identification (see also Fig. 6.15). The $\mathrm{H}_{2}$ feedback control design is formulated as a mixed sensitivity control problem with the addition of a small scalar weight times the noise sensitivity. The small scalar makes the effect of the noise sensitivity negligible, such that the limit in minimizing the input sensitivity is imposed by the communication delays between the real-time system and the motor driver. Tracking will be improved by model-based feed-forward of dynamic forces, which can be predicted accurately due to the deterministic behavior of the mechanics.

\subsection{Experimental validation}

\subsubsection{Parasitic motion}

To confirm the small magnitude of parasitic motion for the butterfly hinge, the encoder (Fig. 6.14 part number 7) is replaced by a capacitive displacement sensor (Lion Precision C6, range: $500 \mu \mathrm{m}$, accuracy: $<0.1 \mu \mathrm{m}$ ) mounted perpendicular to the cylindrical rotor surface of the sensor strip. The displacement sensor is positioned at this location as the axis drift is maximum in the direction perpendicular to the symmetry axis of the butterfly hinge (the $x$-direction as indicated in Fig. 6.7), which is at the location of the encoder. Furthermore, the part of the rotor to which the sensor strip is attached is the only cylindrical surface that is accessible over the full range of motion of 60 degrees of rotation.

Measuring the relative displacement of the rotor over the range of motion includes the combined effect of the parasitic motion of the butterfly hinges (simulated value: $0.1 \mathrm{~mm}$ ), lateral displacement caused by misalignment of the rotor with respect to the rotation axis of the butterfly hinges (eccentricity) and displacement caused by the limited circularity of the reference surface due to manufacturing tolerances. In order to minimize the eccentricity, the butterfly hinges are positioned by dowel pins with a positioning tolerance $<20 \mu \mathrm{m}$. Combined with the bi-directional range of motion of 30 degrees, the lateral displacement caused by eccentricity is $<2 \mu \mathrm{m}$. Furthermore, the reference surface used for the displacement sensor is manufactured by $\mathrm{CNC}$ milling within a tolerance of approximately $10 \mu \mathrm{m}$. Overall, the combined effect of eccentricity and manufacturing tolerances is estimated at $<12 \mu \mathrm{m}$, which is about $10 \%$ of the expected axis drift caused by the deflection of the butterfly hinges.

The total measured displacement of the rotor with respect to its neutral position is $-0.09 \mathrm{~mm}$ at -30 degrees rotation and $0.11 \mathrm{~mm}$ at +30 degrees rotation. Taking 
into account deviations caused by eccentricity and manufacturing tolerances, the magnitude of the parasitic displacement is in line with the simulated values and confirm the ability of the butterfly hinges to maintain the position of the rotation axis in the center of the rotor, including the magnetic forces induced by the rotor.

It has to be noted that the measured (and simulated) values for the parasitic motion are highly repeatable and do not affect the repeatability of the overall system.

\subsubsection{Support stiffness}

To validate the support stiffness of the rotor suspension, the frequency of the first parasitic vibration mode of the end effector is evaluated, which is directly related to the (critical) radial support stiffness. This parasitic vibration mode consists of a translational radial motion approximately parallel to the encoder head on the rotor-hub, which can be detected by the encoder. To determine the stiffness, the frequency response from actuator current to rotation of the rotor is evaluated, which is provided in Fig. 6.15 This frequency response is evaluated close to the maximum deflection angle while still allowing for sufficient range of motion for a sweep signal on the input current. From this data, the first (damped) eigenfrequency in the degree of freedom of the system can be observed at about $5 \mathrm{~Hz}$. At $98 \mathrm{~Hz}$, the first parasitic eigenfrequency can be observed which can be related to the off-axis support stiffness of the system. In combination with the $3.0 \mathrm{~kg}$ mass of the end effector, consisting of the rotor, rotorhub and a part of the butterfly hinges, this provides a radial support stiffness of approximately $1138 \mathrm{~N} / \mathrm{mm}$. This is in good agreement with simulations and confirms the intended high radial stiffness of the butterfly hinges (simulated value $K_{\text {crit }} \approx 1400 \mathrm{~N} / \mathrm{mm}$ at $\theta=25$ degrees, Fig. 6.8b). The slightly lower stiffness values in the measurement can be related to additional compliance of the frame parts, which are disregarded in the simulations.

\subsubsection{Load capacity}

To validate the load capacity, an additional off-centered mass (solid block of aluminum) is attached to the rotor of which the inertia is equivalent to the load in the considered application. This increase of load results in a total inertia of $0.066 \mathrm{kgm}^{2}$ with respect to the rotation axis and a mass of $4.4 \mathrm{~kg}$ with the center of mass located at $65 \mathrm{~mm}$ from the axis of rotation. 


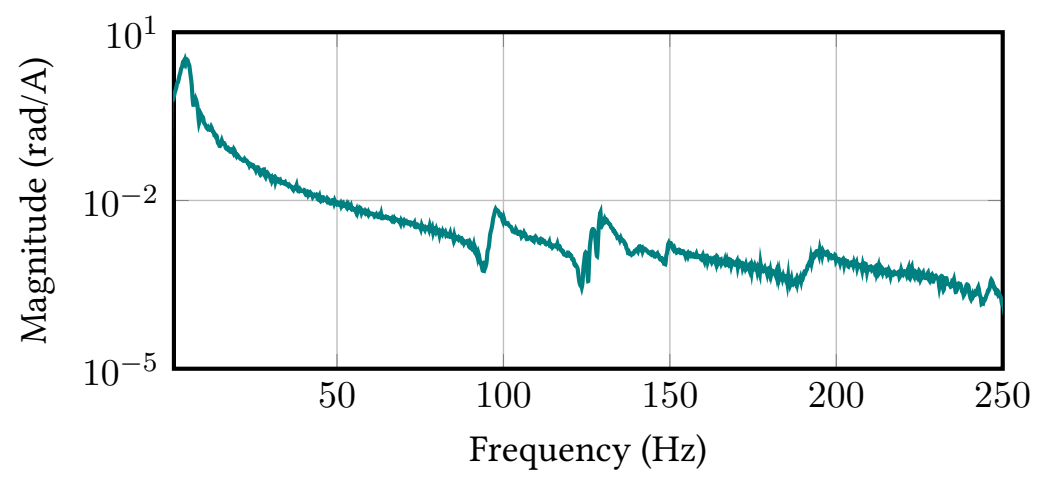

Figure 6.15: Transfer function from current (A) to rotor position (rad) at $\theta=25^{\circ}$
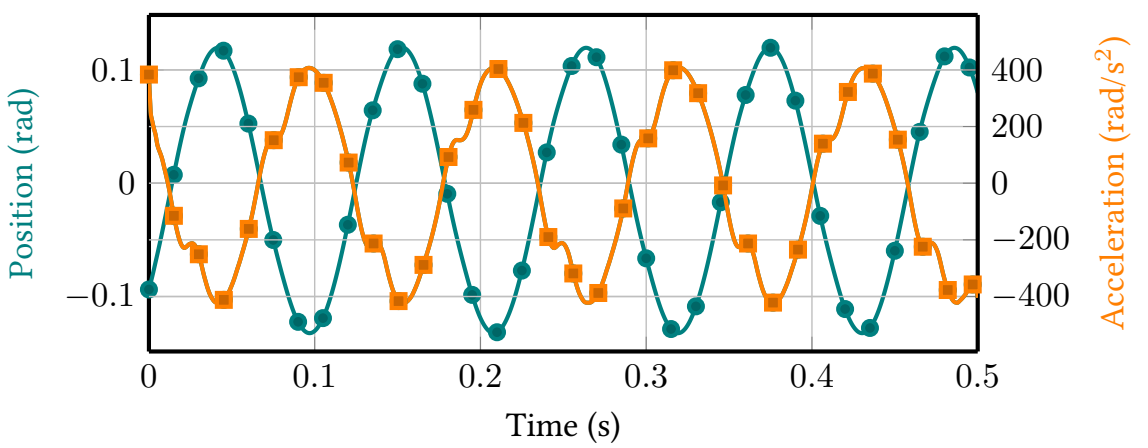

Figure 6.16: Measured position and acceleration of the rotor during load capacity tests. The ripple in accelerations is caused by cogging forces of the actuator (no feedforward/cogging compensation is active during this experiment).

The load capacity is validated by accelerating the mass up to $400 \mathrm{rad} / \mathrm{s}^{2}$, resulting in reaction forces of approximately $115 \mathrm{~N}$ in the radial direction, which is of comparable magnitude as the expected reaction forces in the hexapod application. The measured position and acceleration is presented in Fig. 6.16. Visual inspection of the rotor suspension during the tests show no failure or parasitic deformations which indicate that the actuator suspension is able to withstand the high reaction forces involved. Furthermore, the system has been disassembled and inspected after experiments, showing no signs of damage or contact between the rotor and stator. 


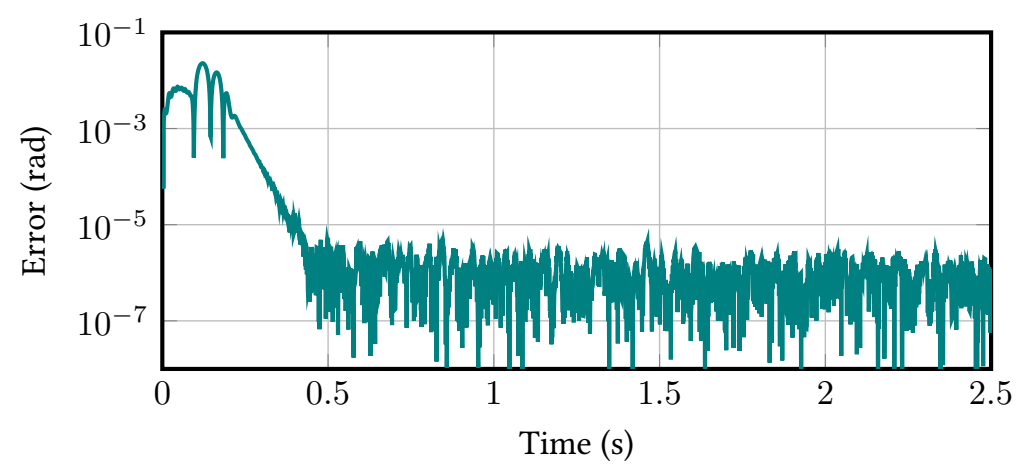

Figure 6.17: Position error for tracking a reference signal moving from $\theta=-22.5^{\circ}$ $(t=0)$ to $\theta=-2.5^{\circ}(t=0.2)$

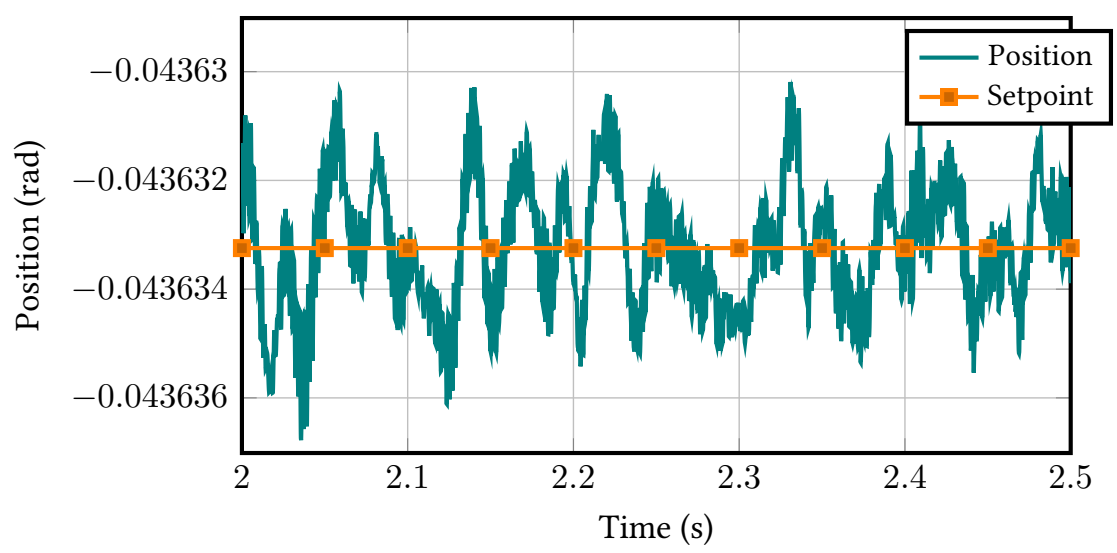

Figure 6.18: Position of the end effector from $t=2$ to $t=2.5$ seconds

\subsubsection{Repeatability}

As the system is free of play and friction (and therefore free of self-locking and limit cycling), the repeatability of the system is directly determined by the standstill performance of the system. To test the repeatability, the rotor is moved to a setpoint while the positioning error is tracked. For the presented case, the rotor is moved from $\theta=-22.5$ to $\theta=-2.5$ degrees in 0.2 seconds. The resulting error for a single movement is provided in Fig. 6.17 where motion starts at $t=$ 0 second. Furthermore, a more detailed view of the position of the rotor between $t=2$ and $t=2.5$ seconds is provided in Fig. 6.18

From the results it can be observed that the position of the rotor has converged to the target position in about 0.5 seconds, after which it fluctuates around the 
target position with an average error of $1.1 \mu \mathrm{rad}$ RMS. These fluctuations in the position are caused by current fluctuations (current ripple) from the motor driver ( $\pm 5 \mathrm{~mA}$ RMS noise), resulting in deviations in the generated torque (motor constant $5.57 \mathrm{Nm} / \mathrm{A}$ ). As stated earlier, due to the absence of friction, no stickslip behavior is present in the flexure-based bearing suspension resulting in no (mechanical) resistance to small current (torque) variations. Therefore, small fluctuations in the provided current directly cause positioning errors of the rotor producing a vibrating motion around the reference position. The driver can be identified as the source of this error as these fluctuations are only present when the motor driver is active.

\subsection{Discussion}

With the tested system a repeatability of $1.1 \mu \mathrm{rad}$ RMS is obtained which is limited by the electronics. In order to reduce positioning errors, several solutions are suggested.

The most straight-forward solution is obtained by reducing the magnitude of the current noise caused by the motor driver. The currently used motor driver has a typical noise level for well-designed commercial motor drivers. Further reducing the output noise would require a dedicated motor driver design, which is costly (especially) considering the high power requirements for the considered actuator. Alternatively, instead of reducing the current noise levels, the sensitivity of the rotor position with respect to current deviations can be reduced. This can be achieved by increasing the total inertia of the system or by reducing the motor constant of the actuator (i.e. selecting a different actuator). However, both solutions reduce the maximum acceleration of the system, with the ratio between maximum acceleration and sensitivity with respect to current noise unchanged.

This ratio can be improved by increasing inertia by means of a counter-mass to balance the weight of the lower arm attached to the rotor (the lower arm in the hexapod application, Fig. 6.1). As the counter-mass compensates for torque required to counteract gravity, less torque is required to maintain a stationary position preserving more torque for accelerating. Furthermore, it results in a more favorable equilibrium position at approximately 0 degrees rotation of the rotor (instead of the equilibrium position beyond -30 degrees, touching the mechanical end stop). At last, balancing results in a location of the center of mass closer to the axis of rotation of the rotor, reducing the radial reaction forces involved with high accelerations. 
For the considered hexapod, fully balancing the static forces (exactly balanced at its neutral position) is feasible by adding a $2.5 \mathrm{~kg}$ counterweight to the rotor. This counterweight would result in approximately twice the effective inertia observed at the actuator, halving the positioning error below $0.6 \mu \mathrm{rad}$ RMS.

\subsection{Conclusion}

A high performance flexure-based suspension for an iron core direct drive torque motor with high radial support stiffness and high load capacity has been designed. The system allows for a 60 degrees range of motion combined with an ultimate torque of $55 \mathrm{Nm}$ and features high repeatability. The repeatability is measured at $1.1 \mu \mathrm{rad}$ RMS positioning error with a load of $0.066 \mathrm{kgm}^{2}$, at present limited by electronics.

For the actuator suspension, a butterfly hinge has been modified and optimized to result in only $0.1 \mathrm{~mm}$ parasitic radial displacement over the range of motion, which limits pull-in forces typical for iron core actuators. The radial support stiffness exceeds $1000 \mathrm{~N} / \mathrm{mm}$ with a radial load capacity of $400 \mathrm{~N}$ at the maximum deflection angle. This provides adequate support stiffness to compensate for the magnetic reaction forces induced by the iron core of the actuator. Furthermore, due to the design of the actuator with a $1 \mathrm{~mm}$ air gap between the rotor and stator, the flexures are inherently protected from excessive (radial) loads by rerouting excessive force through the frame parts, relieving the flexures.

Experimental validations confirm the high support stiffness, load capacity and robustness and prove the applicability of a flexure-based suspension for high torque iron core actuators. 


\title{
T-Flex: A fully flexure-based large range of motion precision hexapod
}

\begin{abstract}
Six degree of freedom manipulation provides full control over position and orientation, essential for many applications. However, six degree of freedom parallel kinematic manipulators (e.g. hexapods) either have a limited range of motion combined with a good repeatability when comprising flexure joints, or they have limited repeatability with a large workspace when using traditional rollingor sliding-element bearings. In this paper, the design and optimization of a fully flexure-based large range of motion precision hexapod robot is presented. The flexure joints have been specifically developed for the purpose of large range of motion and high support stiffness for this manipulator. The obtained system allows for $\pm 100 \mathrm{~mm}$ of translational and more than \pm 10 degrees of rotational range of motion in each direction combined with a base radius of $0.43 \mathrm{~m}$ and a height of $0.42 \mathrm{~m}$. Furthermore, a dedicated flexure-based design for the actuated joints combines high actuation forces with the absence of play and friction, allowing for accelerations exceeding $10 \mathrm{~g}$. Experiments on a prototype validate the sub-micron repeatability, which is merely limited by the selected electronics.
\end{abstract}

\subsection{Introduction}

Various applications require six degree of freedom manipulators. These systems are used in industrial applications such as micro assembly robots, welding robots,

Submitted to: Precision Engineering (M. Naves, M. Nijenhuis, B. Seinhorst, W.B.J. Hakvoort, D.M. Brouwer) 
vibration-isolation platforms, pick-and-place robots and optical alignment systems. For this purpose, often open kinematic serial robot configurations are used [60]. These serial manipulators are used for their large workspace with respect to their footprint. However, due to the open kinematic structure, errors at joints are accumulated and amplified at the end effector, typically allowing for a repeatability up to about $0.01 \mathrm{~mm}$ [50, 51, 100]. Furthermore, due to the serial placement of the actuators, serial robots exhibit large moving masses, resulting in limitations on the maximum accelerations that can be achieved.

In contrast, parallel kinematic configurations consist of six independent kinematic chains connected to the end effector. This allows for the placement of the actuators at the stationary base, resulting in a low moving mass allowing for high accelerations. From a kinematic point of view, parallel robots also allow for a higher repeatability as errors at the joints are averaged, instead of added cumulatively.

High precision parallel kinematic robots comprising traditional bearings allow for a repeatability up to a few tenths of a micrometer [42, 80, 88]. To improve the precision of parallel manipulators, it is required to eliminate sources of play and friction in the joints and actuators. Therefore, the traditional bearings are often replaced by flexure-based equivalents to enable motion, which rely on elastic deformation of slender elements, instead of tribological contacts [80, 82]. However, state-of-the-art parallel manipulators comprising flexure joints suffer from a limited range of motion below $10 \mathrm{~mm}$ of translational travel range. Furthermore, high precision hexapods often rely on walk-drive-type or inertiadrive-type piezoelectric actuators, potentially suffering from wear, limiting the maximum actuation forces and restricting the maximum travel speed to about $10 \mathrm{~mm} / \mathrm{s}$ [54, 93].

Following recent developments in large stroke flexure joints and optimization strategies [19, 27, 32, 68-70, 108], it is anticipated that a flexure-based hexapod with a larger travel range, faster travel speed, higher load capacity and high repeatability can be realized. This paper considers the design and realization of a fully flexure-based six degree-of-freedom parallel manipulator with a large range of motion. A challenging combination of requirements is specified in order to realize a system with unprecedented performance. Flexures allow a competitive repeatability level of $<0.1 \mu \mathrm{m}$, while the range of motion is aimed at a workspace of $100 \times 100 \times 100 \mathrm{~mm}^{3}$. Furthermore, high accelerations of at least $50 \mathrm{~m} / \mathrm{s}^{2}$ are targeted throughout the workspace, with a maximum speed of at least $1 \mathrm{~m} / \mathrm{s}$. Lastly, a maximum vertical load of $10 \mathrm{~kg}$ is specified combined with a mechanism volume below $250 \mathrm{dm}^{3}$ (defined by the volume of the smallest enclosing cylinder). Compared to existing flexure-based precision hexapods, this represents an 
Table 7.1: Specifications of high precision hexapods, a preview of the realized T-Flex specifications and the initial target specifications for the T-Flex. In this table, the travel range is defined as the interdependent travel range unless stated otherwise, showing the maximum travel range where all other axes and the pivot point are at the reference position

\begin{tabular}{|c|c|c|c|c|c|c|c|c|}
\hline Manufacturer & & $\begin{array}{l}\text { H-845.D51 } \\
\text { PI } 80\end{array}$ & $\begin{array}{l}\text { F-206.S } \\
\text { PI } 80\end{array}$ & $\begin{array}{l}\text { Smarpod110 } \\
\text { SmarAct } \\
88\end{array}$ & $\begin{array}{l}\text { Commander6 } \\
\text { Fraunhofer } \\
\text {-Institut } 82\end{array}$ & $\begin{array}{l}\text { HH-30D } \\
\text { ALIO } \\
42\end{array}$ & $\begin{array}{l}\text { T-Flex } \\
\text { University } \\
\text { of Twente }\end{array}$ & $\begin{array}{l}\text { T-Flex } \\
\text { target } \\
\text { spec. }\end{array}$ \\
\hline Flexure-based & & No & Yes & No & Yes & No & Yes & Yes \\
\hline Travel range $X$ & $(\mathrm{~mm})$ & \pm 110 & -8 to +6 & 20 & \pm 4.5 & \pm 30 & \pm 100 & $> \pm 50^{*}$ \\
\hline Travel range $Y$ & $(\mathrm{~mm})$ & \pm 110 & \pm 5.7 & 20 & \pm 4.5 & \pm 30 & \pm 105 & $> \pm 50^{*}$ \\
\hline Travel range $Z$ & $(\mathrm{~mm})$ & \pm 50 & \pm 5.7 & 10 & -3 to 2.1 & \pm 8 & \pm 100 & $> \pm 50^{*}$ \\
\hline Travel range $\theta_{x}$ & (deg) & \pm 15 & \pm 5.7 & 21 & \pm 3.9 & \pm 10 & \pm 21.5 & $> \pm 10$ \\
\hline Travel range $\theta_{y}$ & (deg) & \pm 15 & \pm 6.6 & 24 & \pm 3.4 & \pm 10 & \pm 21.5 & $> \pm 10$ \\
\hline Travel range $\theta_{z}$ & (deg) & \pm 30 & \pm 5.5 & 38 & \pm 7.3 & 360 & \pm 18 & $> \pm 10$ \\
\hline Repeatability & $(\mu \mathrm{m})$ & $2-10$ & 0.3 & 0.2 & 0.05 & \pm 0.1 & 0.35 RMS & $< \pm 0.1$ \\
\hline Max. speed & $(\mathrm{mm} / \mathrm{s})$ & 50 & 10 & 10 & n.a. & $15-50$ & $4000^{* *}$ & $>1000$ \\
\hline Max. load & (kg) & 400 & 1.5 & 0.25 & 0.1 & 5 & 20 & $>10$ \\
\hline Build volume & $\left(\mathrm{dm}^{3}\right)$ & 293 & 7.9 & 0.4 & 1.1 & n.a. & 227 & $<250$ \\
\hline
\end{tabular}

* Simultaneous travel range, showing the maximum travel range where all other axes are also at the maximum travel range

** Merely limited by the maximum travel range and maximum acceleration

increase in travel range up to a factor ten with an unprecedented travel speed up to two orders of magnitude higher. An overview of the specifications of five state-of-the-art precision hexapods, a preview of the realized specifications for the T-Flex and the initially targeted specifications for the T-Flex are provided in Table 7.1

In this paper, the design and optimization of a fully flexure-based large range of motion hexapod is presented. First, in section 7.2, a brief description of the hexapod layout is provided. Next, in section 7.3. an optimization strategy for the hexapod and the optimized design for each individual flexure-based joint is given. The optimization results for the hexapod are presented in section 7.4 and a design for the hexapod, based on the optimization results, is given is section 7.5. An analytic evaluation of the performance of the hexapod throughout the workspace is given in section 7.6. Lastly, a prototype and experimental validation is provided in section 7.7 and 7.8 


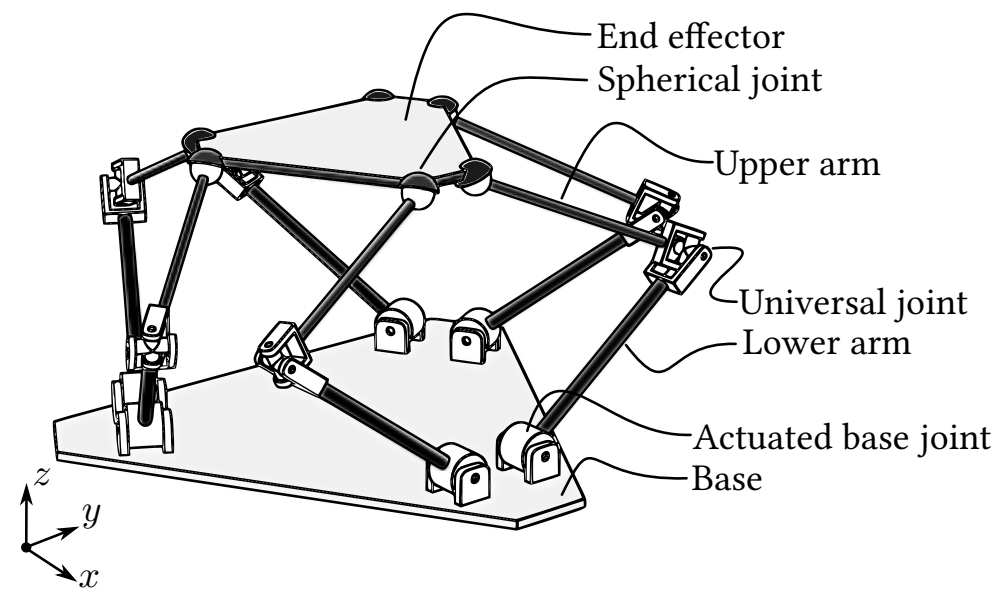

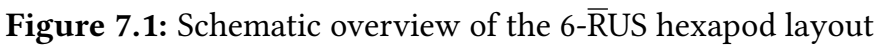

\subsection{Hexapod layout}

Various layouts for six degree of freedom parallel manipulators are described in literature [60], consisting of a combination of prismatic, rotational, universal and spherical joints. For the layout of the hexapod described in this paper, stationary single degree of freedom actuators positioned at the base are preferred to obtain low moving mass. Furthermore, the absence of cables attached to moving parts of the system prevents parasitic bending forces, potentially negating precision. A rotary direct drive actuator is selected as this proved to provide a more compact solution, especially when considering the required large range of motion. The design of the actuator including the flexure-based suspension is discussed in more detail in Chapter 6 and discussed in section 7.3.2.

For releasing the remaining five degrees of freedom of each arm of the hexapod, a passive universal and spherical joint is used connected to each other via an lower and upper arm, resulting in a 6-R̄US type hexapod layout. The design of the spherical and universal joints consist of a folded leafspring based joint design, described in more detail in Chapter 4 [65, 68]. A schematic overview of the 6-RुS layout is provided in Fig. 7.1

The range of motion of this system can be increased by choosing the 6-R̄SS hexapod layout instead. For this layout, the universal joints are replaced by spherical joints. In that case, the rotational motion of the upper-arm around its longitudinal axis is distributed over both spherical joints, resulting in an increase of the workspace mainly in the rotational direction around the vertical $z$-axis. 
However, this increased range of motion comes at the cost of internal (underconstrained) degrees of freedom of the upper arms. Since flexures provide small stiffness in their degrees of freedom, this underconstrained body can impose unwanted (low frequency) internal vibrations which may jeopardize controller bandwidth and positioning accuracy of the system.

For the spherical and universal joints of the hexapod, identical designs are chosen, with the exception of a single additional flexure for the universal joints to constrain one of the rotational degrees of freedom. As this additional flexure in the universal joint mainly affects the rotational stiffness at the upper arm along its longitudinal axis (the stiffness contributing to the internal eigenfrequency) without affecting other stiffness properties of the system, the behavior of a 6-

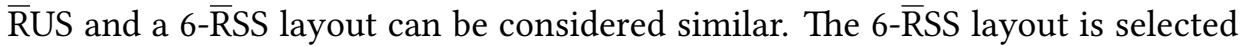
for the optimization of the hexapod described in section 7.3 , which can easily be converted to a $6-\overline{\mathrm{R}} U S$ layout.

\subsection{Optimization}

The pose-dependent stiffness and limited range of motion of flexure joints result in complex mechanical behavior of flexure-based systems [19,59, 74], especially when considering a large range of motion. Therefore, manually designing a flexure-based hexapod is not straight forward. To overcome this, an optimization algorithm is used to optimize the design.

\subsubsection{Hexapod optimization strategy}

For the optimization, the relevant mechanical behavior is simulated in the most critically deflected configurations of the system. These critical configurations are given by the eight extrema of the $\pm 50 \mathrm{~mm}$ of travel range in each degree of freedom (e.g. $x=50 \mathrm{~mm}, y=50 \mathrm{~mm}, z=50 \mathrm{~mm}$ ). However, including the full elastic behavior of all 18 flexure-based joints over the full large range of motion would imply high complexity and large computational costs that renders optimization impractical. Furthermore, the high number of design variables involved will result in problems with respect to convergence to the optimal solution. To reduce complexity of the optimization problem, it is divided into two separate optimizations. First, the design of each flexure-based joint is individually optimized, taking into account the detailed elastic behavior of the flexures. Next, the geometric design of the hexapod is optimized by using a reduced model with the non-linear stiffness properties of each joint lumped between the links of 
the hexapod. The stiffness properties of the joints are derived from the earlier conducted optimization and simulation of the individual flexure joints. Parasitic motion of the flexure-based joints, which is defined by motion which does not correspond to the intended motion direction when deflected, is disregarded as its influence on system-level performance is only small. The decrease in support stiffness caused by deflection in the degrees of freedom is taken into account as this contribution is significant.

As the designs of the joints are optimized separately and do not change in the optimization loop for the geometry of the hexapod, no computational effort is required to compute the joint's stiffness properties during this second optimization. Therefore, the mechanism state, i.e. the rotations of all joints, can be derived from kinematics only and the computational cost for evaluating the performance of the hexapod for a specific set of design parameters is only small (i.e. a few seconds on a conventional computer system).

Lumping of the compliance properties of the flexure-based joints is done by modeling them as two-node elements. To obtain the compliance properties, which are a function of motion of the joints in the degrees of freedom, a third-order polynomial is fitted through the compliance values obtained from the initial optimization and simulation of the individual joints. The six deformation modes of the two-node elements are defined by

$$
\begin{array}{rlrl}
\epsilon_{x} & =x^{q}-x^{p} & \epsilon_{\theta_{x}} & =\theta_{x}^{q}-\theta_{x}^{p} \\
\epsilon_{y}=y^{q}-y^{p} & \epsilon_{\theta_{y}}=\theta_{y}^{q}-\theta_{y}^{p} \\
\epsilon_{z}=z^{q}-z^{p} & \epsilon_{\theta_{z}}=\theta_{z}^{q}-\theta_{z}^{p}
\end{array}
$$

with the superscripts $p$ and $q$ indicating the two nodes of the element each connected to a different arm of the hexapod. Furthermore, $x, y$ and $z$ provide the position of each node and $\theta_{x}, \theta_{y}$ and $\theta_{z}$ its orientation in $z-y-x$ Euler angles with respect to a local reference frame. Note that both nodes are initially aligned and positioned identically, yielding zero deformation. The corresponding stress resultants (reaction forces) are provided by

$$
\begin{array}{ll}
\sigma_{x}=\epsilon_{x} / C_{x} & \sigma_{\theta_{x}}=\epsilon_{\theta_{x}} / C_{\theta_{x}} \\
\sigma_{y}=\epsilon_{y} / C_{y} & \sigma_{\theta_{y}}=\epsilon_{\theta_{y}} / C_{\theta_{y}} \\
\sigma_{z}=\epsilon_{z} / C_{z} & \sigma_{\theta_{z}}=\epsilon_{\theta_{z}} / C_{\theta_{z}}
\end{array}
$$

with $C$ the compliance in each degree of freedom of the element. For example, for a spherical joint, $C_{\theta_{x}}, C_{\theta_{y}}$ and $C_{\theta_{z}}$ represent the compliances in the three degrees of freedom of the joint and $C_{x}, C_{y}$ and $C_{z}$ provide the compliances in 
the supposedly stiff support directions. Note that the compliance values of the individual joints are not constant over the range of motion and depend on the deflection angle(s) in the degrees of freedom of the joints, which will be discussed in more detail in section 7.3.2 and 7.3.3 Furthermore, the compliance properties are evaluated with respect to the center of the joints, which approximates the center of compliance. Therefore, coupling between the deformation modes is only small and negligible.

\subsubsection{Actuated revolute base joint optimization}

\section{Actuator selection}

For the actuation of the revolute base joints, an actuator is required that does not jeopardize the pursued precision while allowing for a large range of motion, large velocities and large accelerations. For precision systems, piezo-based walkdrive-type or inertia-drive-type actuators can be used as they provide highly deterministic behavior combined with a high positioning resolution. However, these type of actuators typically suffer from a limited maximum motion velocity (typically $<10 \mathrm{~mm} / \mathrm{s})$ and limited actuation forces $(<15 \mathrm{~N})$. Furthermore, they suffer from wear and a limited total travel range (e.g. $\leq 10 \mathrm{~km}$ ), which limits their service life especially when considering large range of motion applications.

In order to overcome these limitations, a permanent magnet torque motor with iron core is selected (Tecnotion's QTR-A-133-60-N torque motor [94]). This actuator allows for a high ultimate ${ }^{1}$ output torque of $55 \mathrm{Nm}$ and a non-restrictive maximum velocity of $230 \mathrm{rad} / \mathrm{s}$. The flexure-based revolute joint supporting the rotor of the actuator is designed to limit parasitic reaction forces caused by the iron core. Finally, the absence of self locking in the selected actuator, or in fact the absence of any friction in the drive train, renders the system back-drivable. Therefore, this actuator enables the sensing of interaction forces with respect to the environment by using the motor current and position feedback only, which is not possible for self-locking piezo-based systems.

The rotation of the rotor is measured via Heidenhain's LIC 4119 encoder with a $1 \mathrm{~nm}$ measuring step, resulting in a rotational resolution of $13 \mathrm{nrad}$. The actuator is controlled with Kollmorgen's industrial class AKD-P00306 servo drive which can provide a $1 \mathrm{~mA}$ current feedback. In combination with the motor constant of the actuator, this allows for an $6 \mathrm{Nmm}$ torque feedback resolution.

1 At ultimate torque the temperature of the coil increases by $20^{\circ} \mathrm{C} / \mathrm{s}$. The maximum duration at which this torque can be maintained depends on thermal properties of the coils housing. 


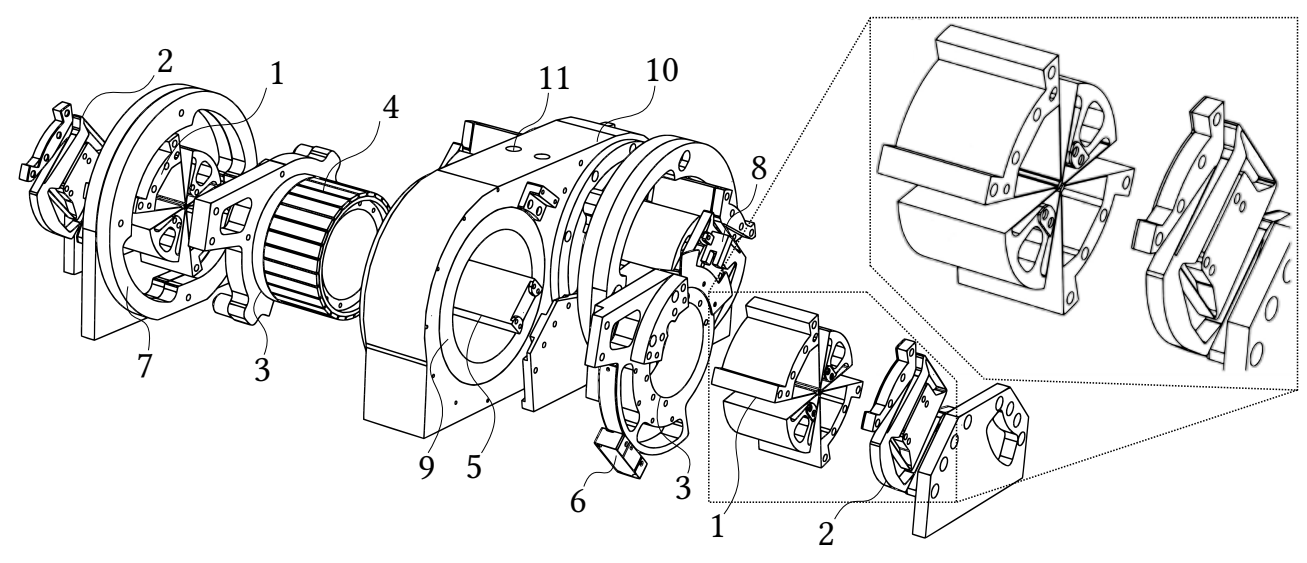

Figure 7.2: Exploded view of actuator suspension. 1) Butterfly hinge 2) Slaving mechanism 3) Rotor-hub 4) Permanent magnets of the rotor 5) Body coupling intermediate bodies of both butterfly hinges 6) Encoder 7) Mechanical stop 8) Optical switch 9) Actuator's stator 10) Stator housing 11) Cooling channels [70].

\section{Base joint optimization}

The actuated revolute joint at the base is based on the butterfly hinge design [33] and optimized for maximum off-axis radial support stiffness. The butterfly hinge design is selected as it features a low shift of the rotation axis, which is required to maintain good alignment between the rotor and the stator. A detailed design drawing of this flexure-based actuator suspension is provided in Fig. 7.2 consisting of two butterfly hinges placed in parallel at each side of the rotor of the actuator.

For the optimization of the butterfly hinges, an adapted Nelder-Mead based shape optimization algorithm [69] is used. In the optimization loop, the performance is evaluated over the range of motion with the flexible multibody software SPACAR [45]. The hinge is modeled with a series of interconnected nonlinear 3D finite beam elements, which include the geometric nonlinearities to capture the relevant behavior of the flexures. For the optimization objective, the off-axis radial support stiffness is selected in order to maximize support stiffness for the lower arm and to provide resistance to the magnetic forces between the permanent magnets and the iron core. A stainless tooling steel is selected with an E-modulus of $200 \mathrm{GPa}$ and a maximum allowable fatigue stress of $600 \mathrm{MPa}$ (about $40 \%$ of the yield stress). Furthermore, a range of motion of \pm 25 degrees is considered, which is slightly less than the \pm 30 degrees considered in Chapter 6 The range of motion is decreased in order to ensure all joints utilize their full range of motion through- 


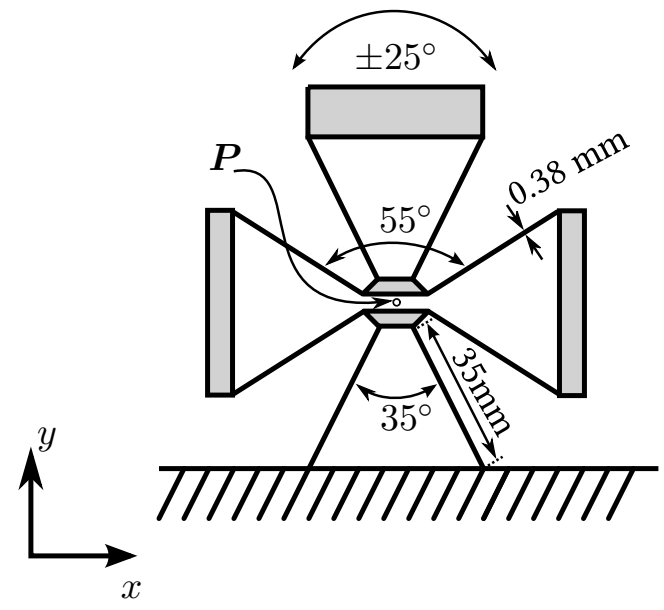

Figure 7.3: Schematic overview of the optimized dimensions for the butterfly hinge

out the considered workspace. The width of each butterfly hinge (the dimension measured parallel to the rotation axis) is limited to $50 \mathrm{~mm}$. Furthermore, a center to center distance of $160 \mathrm{~mm}$ between both butterfly hinges is considered, which is required for the space claim for the actuator. A schematic overview of the butterfly hinge with the optimized dimensions is provided in Fig. 7.3 , with each flexure given the same dimensions and $\boldsymbol{P}$ indicating the location of the rotation axis.

\section{Base joint compliance properties}

The compliance of an individual butterfly hinge is evaluated with respect to the center of the joint (at point $\boldsymbol{P}$, Fig. 7.3), which results in off-diagonal terms in the compliance matrix that are at least an order of magnitude smaller and therefore negligible. Hence, the compliance of a single butterfly hinge can be approximated by diagonal compliance matrix. As the resulting compliance of the butterfly hinge depends strongly on the rotation angle of the hinge in the degree of freedom $\left(\theta_{r}\right)$, a third-order polynomial is fitted through the numerically obtained compliance values to capture the variation. As the behavior of the butterfly hinge is equal for positive and negative rotation angles, each individual compliance term is approximated by

$$
C=p_{0}+p_{1}\left|\theta_{r}\right|+p_{2}\left|\theta_{r}\right|^{2}+p_{3}\left|\theta_{r}\right|^{3}
$$

with $\theta_{r}$ the rotation angle in degrees. An overview of the fitted coefficients is provided in Table 7.2 . The compliance values for the translational and rotational 


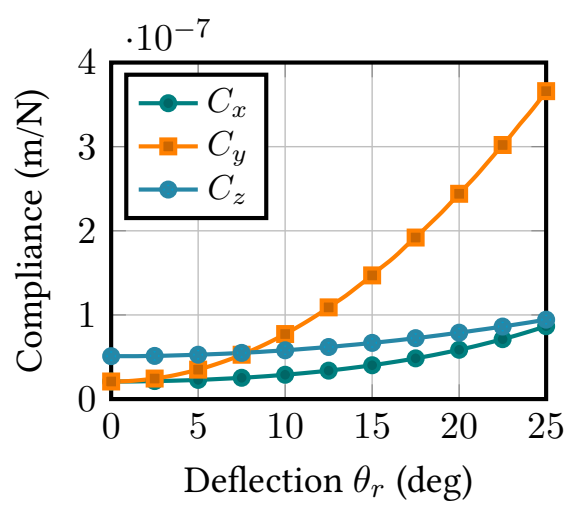

(a)

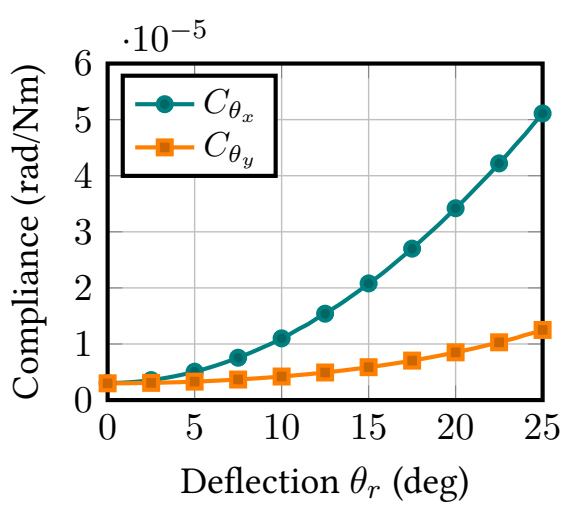

(b)

Figure 7.4: (a) Translational and (b) rotational compliance values of the optimized revolute base joint

Table 7.2: Interpolation values for the compliance in the support directions of the base joint with $\mathrm{R}^{2}>0.99$

\begin{tabular}{ccccc} 
& $p_{0}$ & $p_{1}$ & $p_{2}$ & $p_{3}$ \\
\hline \hline$C_{x}$ & $2.06 \cdot 10^{-8}$ & $2.22 \cdot 10^{-10}$ & $3.59 \cdot 10^{-11}$ & $2.42 \cdot 10^{-12}$ \\
$C_{y}$ & $2.10 \cdot 10^{-8}$ & $-8.63 \cdot 10^{-11}$ & $5.84 \cdot 10^{-10}$ & $-1.14 \cdot 10^{-12}$ \\
$C_{z}$ & $5.09 \cdot 10^{-8}$ & $-5.00 \cdot 10^{-12}$ & $7.22 \cdot 10^{-11}$ & $-9.85 \cdot 10^{-14}$ \\
$C_{\theta_{x}}$ & $3.05 \cdot 10^{-6}$ & $-1.72 \cdot 10^{-10}$ & $8.14 \cdot 10^{-8}$ & $-1.80 \cdot 10^{-10}$ \\
$C_{\theta_{y}}$ & $2.95 \cdot 10^{-6}$ & $3.40 \cdot 10^{-8}$ & $5.72 \cdot 10^{-9}$ & $3.25 \cdot 10^{-10}$ \\
\hline
\end{tabular}

directions are provided in Fig. 7.4 Note that the compliance in the degree of freedom is almost constant and of a different order of magnitude $(0.27 \mathrm{rad} / \mathrm{Nm})$, and therefore not provided in this figure.

\subsubsection{Spherical/universal joint optimization}

The designs of the spherical and universal joints are based on the two stage serially stacked spherical joint concept described in [68], optimized to maximize support stiffness in the load carrying direction (along the longitudinal direction of the upper arm, Fig.7.1). A range of tip-tilt motion of \pm 25 degrees is considered, combined with a \pm 10 degrees of pan motion for the spherical joint. These values for the maximum deflection angles are deduced from the maximum range of motion which could be obtained by this joint design while maintaining high 


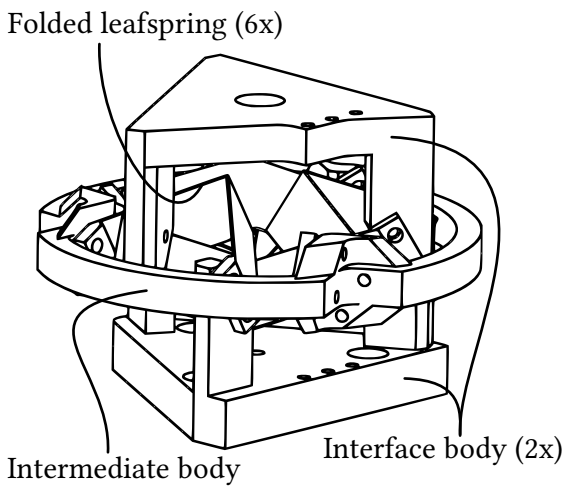

(a)

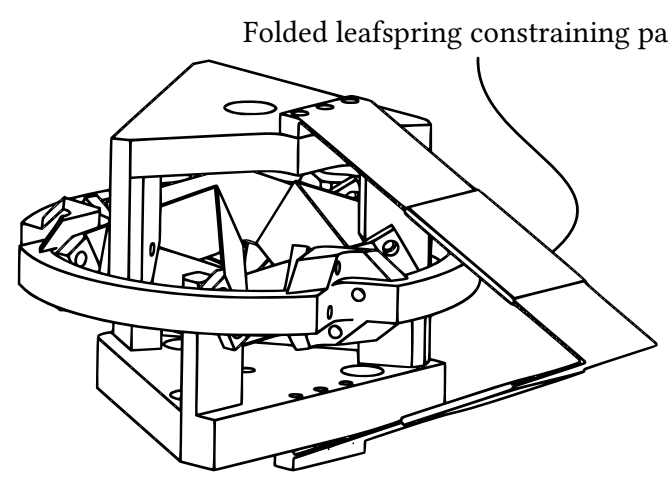

(b)

Figure 7.5: Design drawing of optimized two stage serial stacked (a) spherical joint and (b) universal joint with pan motion constrained by an extra folded leafspring

support stiffness combined with a small envelope ( $<90 \mathrm{~mm}$ diameter). A detailed design drawing of the flexure-based universal and spherical joint is provided in Fig. 7.5 It has to be noted that the design of the universal and spherical joint is identical, with the exception of a single additional flexure constraining one of the rotational degrees of freedom for the universal joint.

For optimization of the spherical joint the same optimization strategy as for the revolute base joint is used. For the optimization objective, the stiffness along the symmetry axis is considered as this stiffness contributes to the observed stiffness at the end effector of the hexapod. For material, stainless tooling steel is again selected with an E-modulus of $200 \mathrm{GPa}$ and a maximum allowable stress of $600 \mathrm{MPa}$. To constrain the footprint of the spherical joint, the diameter is limited to $90 \mathrm{~mm}$. A schematic overview of the spherical joint with the optimized dimensions is provided in figure 7.6 with each flexure given the same dimensions.

\section{Spherical/universal joint compliance properties}

The compliance properties of the universal and spherical joint are also identified by fitting third-order polynomials through the numerically obtained compliance values. However, as motion in multiple degrees of freedom is allowed, the compliance properties are a function of this multi-dimensional rotation. In [68] it was observed that the support stiffness along the symmetry axis of the spherical joint was mostly affected by the tip or tilt angle, which we have captured in a single tilt angle perpendicular to the symmetry axis irrespective of pan motion. Therefore, the compliance can be approximated by

$$
C=p_{0}+p_{1} \theta_{t}+p_{2} \theta_{t}^{2}+p_{3} \theta_{t}^{3}
$$




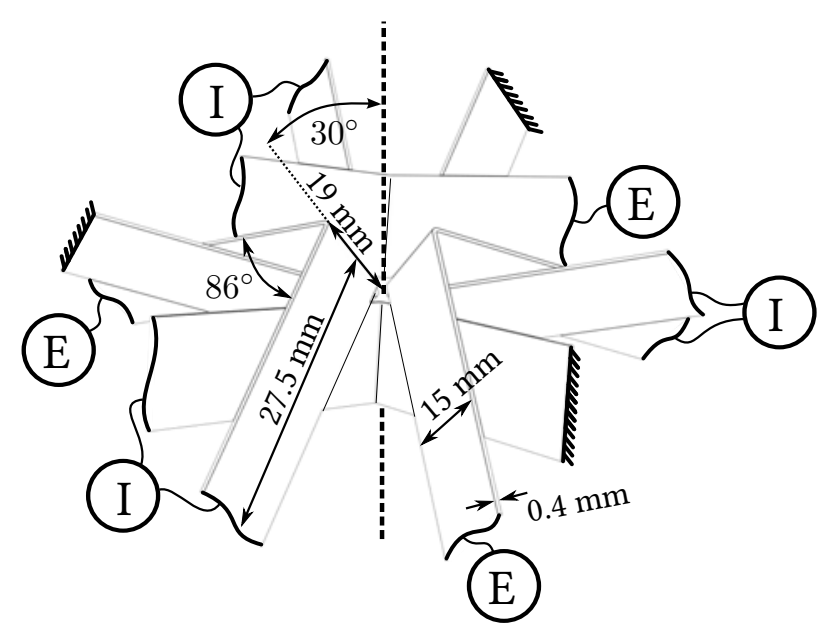

Figure 7.6: Schematic overview of the flexures of the two stage serial stacked spherical flexure joint with the optimal dimensions. The dashed line provides the symmetry axis, $\mathrm{E}$ the connection with the interface body and I the connection with an intermediate body.

with $\theta_{t}$ the tilt angle in degrees. An overview of the fitted coefficients is provided in Table 7.3 The compliance values for the translational directions are shown in Fig. 7.7. Note that the compliance values in $x$ - and $y$-direction are taken equal due to the rotational symmetry of the spherical joint. Furthermore, as the frame compliance of the spherical joint has a significant contribution to the overall compliance, additional frame compliance is taken into account. To assess this frame compliance, compliance of the joint in undeflected state with a rigid and a flexible frame is evaluated by means of a FEM simulation. By comparing both compliance values and by taking the frame and flexure compliance in series, the frame compliance is estimated at $1.25 \cdot 10^{-6} \mathrm{~m} / \mathrm{N}$ in the vertical direction and $1.25 \cdot 10^{-5} \mathrm{~m} / \mathrm{N}$ in the directions perpendicular to it.

Table 7.3: Interpolation values for the compliance of the spherical joint with $\mathrm{R}^{2}>0.99$

\begin{tabular}{lcccc} 
& $p_{0}$ & $p_{1}$ & $p_{2}$ & $p_{3}$ \\
\hline \hline$C_{x / y}$ & $3.85 \cdot 10^{-6}$ & $-2.88 \cdot 10^{-9}$ & $1.13 \cdot 10^{-8}$ & $-3.76 \cdot 10^{-11}$ \\
$C_{z}$ & $6.58 \cdot 10^{-7}$ & $-2.93 \cdot 10^{-9}$ & $8.59 \cdot 10^{-10}$ & $4.56 \cdot 10^{-11}$ \\
\hline
\end{tabular}




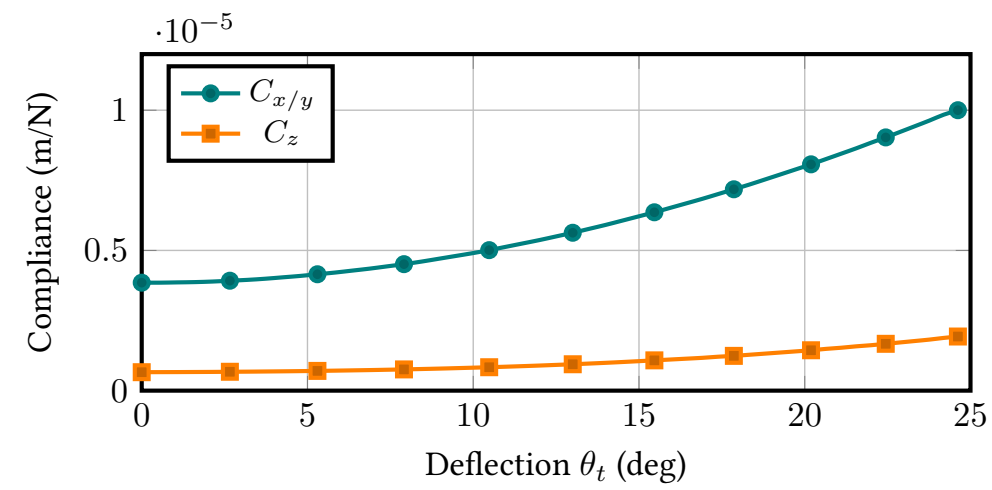

Figure 7.7: Translational compliance of optimized spherical joint

\subsubsection{Hexapod design optimization}

For high positioning accuracy of the hexapod, a high controller bandwidth is desired to obtain good dynamic performance and to suppress disturbances, e.g. current noise caused by the motor drivers. As the mass and inertia properties of the individual components are not known beforehand and depend strongly on the geometry of hexapod, accurately evaluating this parasitic eigenfrequency in the optimization loop is hard. However, as the stiffness observed at the end effector is strongly related to the critical parasitic eigenfrequency, this stiffness can be used instead. Furthermore, when the system is controlled via the encoders on the actuators, this stiffness is crucial for obtaining high precision at the end effector when subjected to external forces and disturbances. Therefore, the translational compliance of the end effector (given locked actuators in the actuated direction) in the most compliant direction is used as the optimization objective.

For evaluating the performance of the hexapod given a specific set of design parameters, first an inverse kinematic rigid body analysis is conducted for which the end effector is positioned at each extremum of the workspace of $100 \times 100 \times$ $100 \mathrm{~mm}^{3}$. Hereby, the pose of the hexapod and the deflection angle of each individual joint is determined. Next, the obtained support stiffness at the end effector is evaluated by taking into account the lumped compliance properties of all flexure-based joints by modeling them as the two-node flexible elements according to Eqs. (7.1) and (7.2). Note that the stiffness values of the joints strongly depend on their deflection angles in the degrees of freedom of the joints, which are obtained from the inverse kinematics. A schematic overview of this lumped model is provided in Fig. 7.8 with each spring representing a six dimensional lumped stiffness. Compliance in the frame parts is assumed negligible 


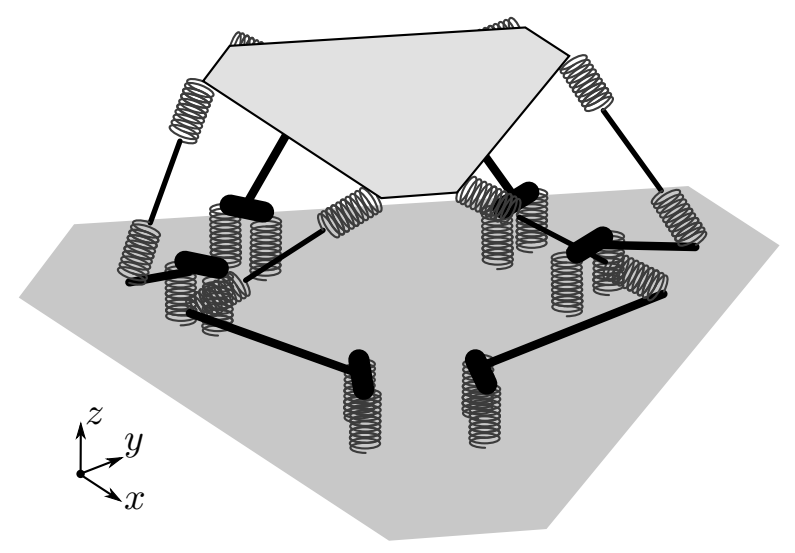

Figure 7.8: Schematic overview of the lumped elastic hexapod model

with respect to the compliance of the flexure-based joints, with exception of the frame parts of the spherical joints whose stiffness has proven to have a significant contribution.

For the optimization of the geometry of the hexapod, the optimization strategy provided in section 7.3.2 is used. The geometry of the hexapod is described by eight design parameters $\left(R_{1}, R_{2}, R_{3}, \beta_{1}, \beta_{2}, \beta_{3}, H_{1}, H_{2}\right)$ which provide the position of each of the joints in the parallel chains, schematically illustrated in Fig. 7.9 The base joints are placed on a circle with radius $R_{1}$, with each pair

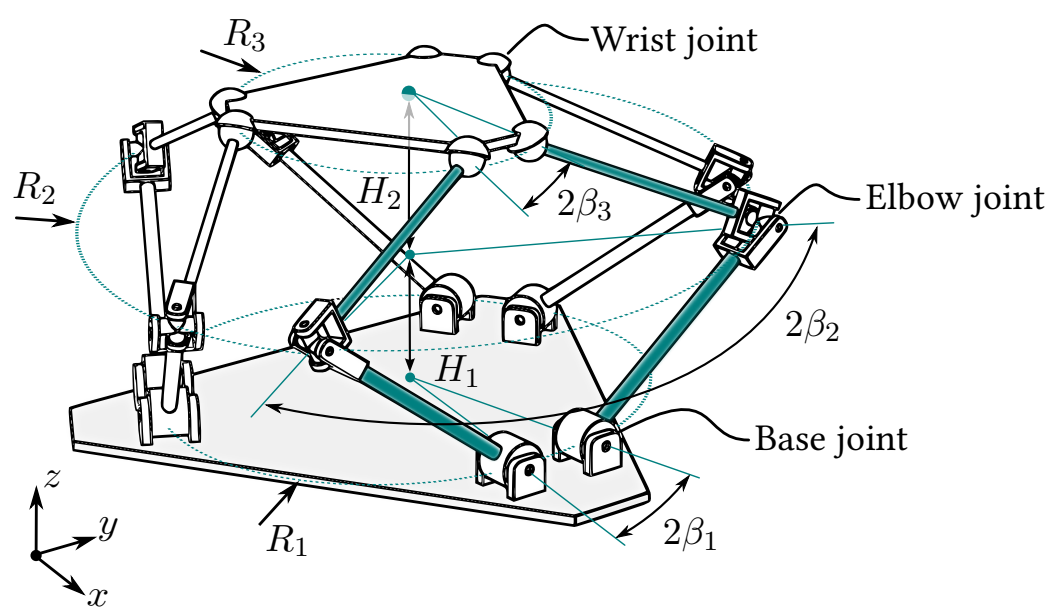

Figure 7.9: Schematic overview of the design parameters with a single "arm pair" colored in teal 
of arms separated by angle $2 \beta_{1}$ between them. Similarly, the elbow and wrist joints are placed on circles with radii $R_{2}$ and $R_{3}$, which are separated by each other with angles $2 \beta_{2}$ and $2 \beta_{3}$. Lastly, the height of the elbow and wrist joints are specified by $H_{1}$ and $H_{2}$.

Furthermore, constraints are specified for the optimization in order to limit the deflection angle of the individual joints, limiting the rotation angle of the revolute base joints to 25 degrees and the rotations of the elbow and wrist joints to 25 degrees tip-tilt. Rotation around the symmetry axis of the spherical joints, providing pan motion, is limited to 10 degrees. Collision between the six parallel chains of the hexapod is avoided by putting a constraint on the minimum distance between each of the joints. A minimum distance between the centers of the base joints of $150 \mathrm{~mm}$ is required which is dictated by the size of the actuators and their housing. Furthermore, given the diameter of $90 \mathrm{~mm}$ of the universal and spherical joints, a minimal distance of $90 \mathrm{~mm}$ between them is required. Note that the distance between the elbow joints depends on the pose of the hexapod, and therefore needs to be evaluated throughout the workspace. Lastly, a limitation on the maximum mechanism volume (in undeflected state) of $0.25 \mathrm{~m}^{3}$ is added, defined by the volume of the smallest enclosing cylinder including the diameter of the joints.

\subsection{Optimization results}

An initial optimization is conducted by taking into account all eight design parameters independently. However, considering each design parameter as an independent degree of freedom has shown to result in inconvenient values that complicate the mechanical design. Hereby, the design becomes more complex, costly and possibly suffers from increased mass and reduced stiffness of the frame parts. Furthermore, optimizations on the full set of design parameters result in a wide range of local optima with almost similar performance, which indicates redundancy in the design freedom. This means that the number of independent design parameters can be reduced without excluding good mechanism designs from the optimization problem. For this purpose, design parameters are iteratively eliminated by choosing convenient constraint relations between some of the design parameters, which will be detailed in the next section. For each iteration, the optimal solution is provided in Table 7.4 


\subsubsection{Iterations}

\section{Iteration 1}

The first optimization, considering all eight independent design parameters, provides solutions at which the base and wrist joints of each arm pair are positioned in a single vertical plane. In other words, $R_{1} \cos \left(\beta_{1}\right)=R_{3} \cos \left(\beta_{3}\right)$, which can be rewritten to a constraint function for $R_{3}$

$$
R_{3}=R_{1} \frac{\cos \left(\beta_{1}\right)}{\cos \left(\beta_{3}\right)}
$$

eliminating $R_{3}$ as an independent design variable.

\section{Iteration 2}

Optimization results using constraint equation (7.5) yield solutions with near parallel rotation axis of the base joints of a single arm-pair. By demanding both rotation axes to be parallel, the design of the actuators can be simplified. Ensuring the rotation axis of the two base hinges of an arm pair to be parallel can be obtained by

$$
R_{2}=R_{1} \frac{\cos \left(\beta_{1}\right)}{\cos \left(\beta_{2}\right)}
$$

eliminating $R_{2}$ as an independent design variable.

\section{Iteration 3}

Optimization results using constraint equations (7.5) and (7.6) show convergence to solutions for which the wrist joints of a single arm-pair are placed as close to each other as possible. Taking into account the required offset of $90 \mathrm{~mm}$ between the spherical joints at the platform, we can eliminate $\beta_{3}$ as an independent design variable by

$$
\beta_{3}=\arcsin \left(\frac{90 \mathrm{~mm}}{2 R_{3}}\right)
$$

Note that the design freedom obtained this way (the design space) shows strong similarities with the Stewart platform [60] when considering the location of the joints. More specifically, the universal and spherical joint of a single arm are in a vertical plane perpendicular to the ground. Furthermore, the spherical joints at the end effector of each arm pair are positioned close together whereas the lower spherical joints are positioned at close distance to the neighboring arm-pair. 


\section{Iteration 4}

Optimization results using constraint equations (7.5), 7.6) and (7.7) show that the system benefits from base joints located as close together as possible. Therefore, the distance between the base joints can be chosen equal to $150 \mathrm{~mm}$ by means of

$$
\beta_{1}=\arcsin \left(\frac{150 \mathrm{~mm}}{2 R_{1}}\right)
$$

eliminating $\beta_{1}$ as an independent variable.

\subsubsection{Discussion}

From the obtained optimization results shown in Table 7.4 it can be concluded that the additional constraint equations do not result in a penalty on the performance of the overall design, despite the strong reduction in the freedom space of the optimization problem. For some of the iterations, even a small increase in performance is obtained because the convergence improves when the set of design parameters is reduced. The final dimensions of the hexapod (last column of Table 7.4) have been slightly adjusted to improve manufacturability and to prevent collision between the lower arm and the ground surface.

Table 7.4: Optimization results for each iteration. The asterisk marks the dependent design parameters following from the constraint equations of section 7.4 The last column provides the set of design parameters used for the final design.

\begin{tabular}{lllllll} 
Iteration & 0 & 1 & 2 & 3 & 4 & - \\
\hline \hline Performance (N/mm) & 340 & 346 & 341 & 350 & 346 & 299 \\
\hline$R_{1}(\mathrm{~mm})$ & 286 & 266 & 281 & 271 & 266 & 255 \\
$R_{2}(\mathrm{~mm})$ & 413 & 415 & $488^{*}$ & $427^{*}$ & $417^{*}$ & $387^{*}$ \\
$R_{3}(\mathrm{~mm})$ & 285 & $291^{*}$ & $279^{*}$ & $265^{*}$ & $259^{*}$ & $248^{*}$ \\
$\beta_{1}(\mathrm{deg})$ & 16.5 & 16.8 & 15.8 & 16.0 & $16.4^{*}$ & $17.2^{*}$ \\
$\beta_{2}(\mathrm{deg})$ & 52.5 & 52.2 & 52.9 & 52.3 & 52.4 & 50.9 \\
$\beta_{3}(\mathrm{deg})$ & 13.8 & 9.5 & 14.3 & $9.8^{*}$ & $10.0^{*}$ & $10.6^{*}$ \\
$H_{1}(\mathrm{~mm})$ & 79 & 80 & 63 & 71 & 81 & 100 \\
$H_{2}(\mathrm{~mm})$ & 183 & 206 & 193 & 278 & 195 & 190 \\
\hline
\end{tabular}




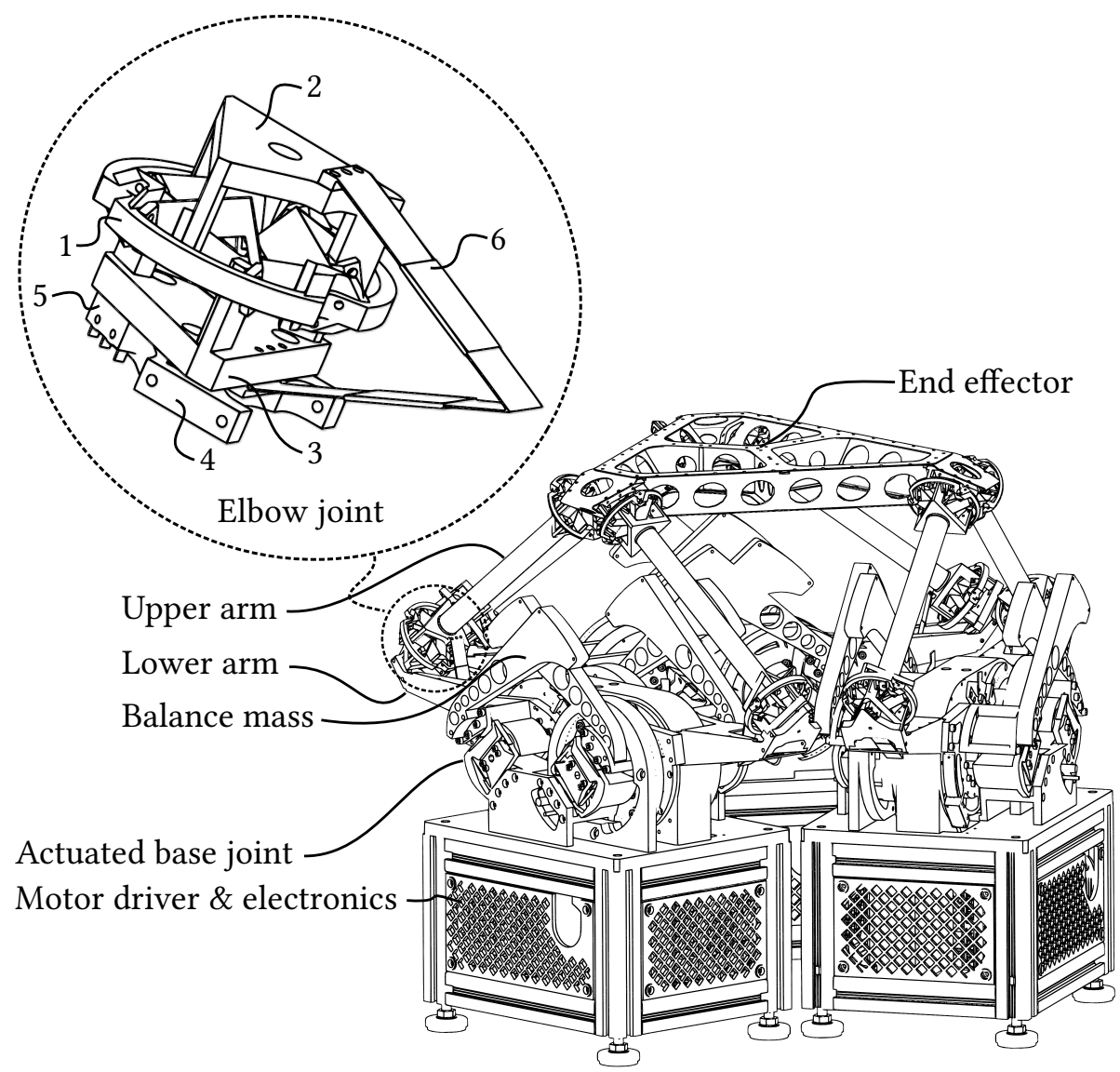

Figure 7.10: Design drawing of the T-Flex with enlarged view of a universal joint. 1) Intermediate frame part 2) frame part attached to upper arm 3) frame part attached to lower arm 4) frame part of lower arm 5) overload switch 6) optional folded leafspring to eliminate the unconstrained degree of freedom of upper arm

\subsection{System design}

Based on the geometry obtained from the optimizations, a prototype of the hexapod has been designed. A design drawing is provided in Fig. 7.10. Furthermore, a front and top view with the main dimensions are provided in Figs. 7.11 and 7.12

Each pair of actuated revolute base joints with parallel rotation axes is combined into a single assembly. The electronics for each actuator pair are placed in a single electronic box placed underneath the actuators. Furthermore, two steel balance masses are attached to each actuator to counteract gravity and to ensure 


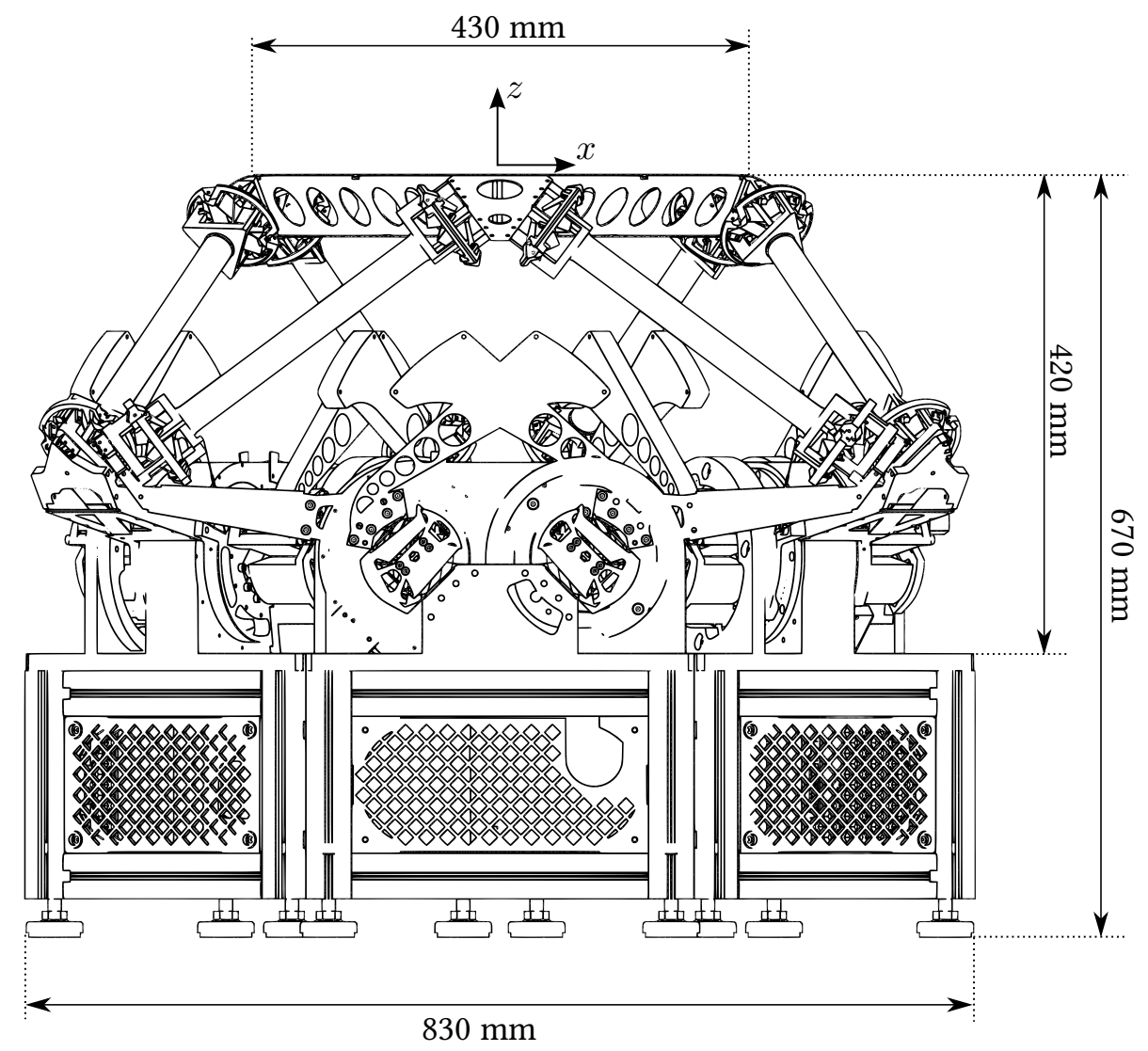

Figure 7.11: Front view of the T-Flex with the main dimensions

that the neutral position of the hexapod is approximately at the center of its workspace. The balance masses do add to the inertia of the lower arms, lowering the achievable accelerations. However, additional inertia simultaneously reduces the sensitivity to current noise, making the trade-off worthwhile.

The lower arm is attached to the revolute base joint. It provides the connection with the first elbow joint and consists of a hollow aluminum structure. The connection between the lower arm and this joint is equipped with a mechanical overload mechanism fitted between the frame part of the lower arm (part 4, figure 7.10) and the frame part of the spherical joint (part 3). Those parts are attached to each other by means of a kinematic coupling of the Maxwell type consisting of three V-shaped grooves oriented to the center of the part, mated to a counter part with three spherical surfaces preloaded by a permanent magnet. The preload force is adjusted such that is disconnects at excessive moments in order to pre- 


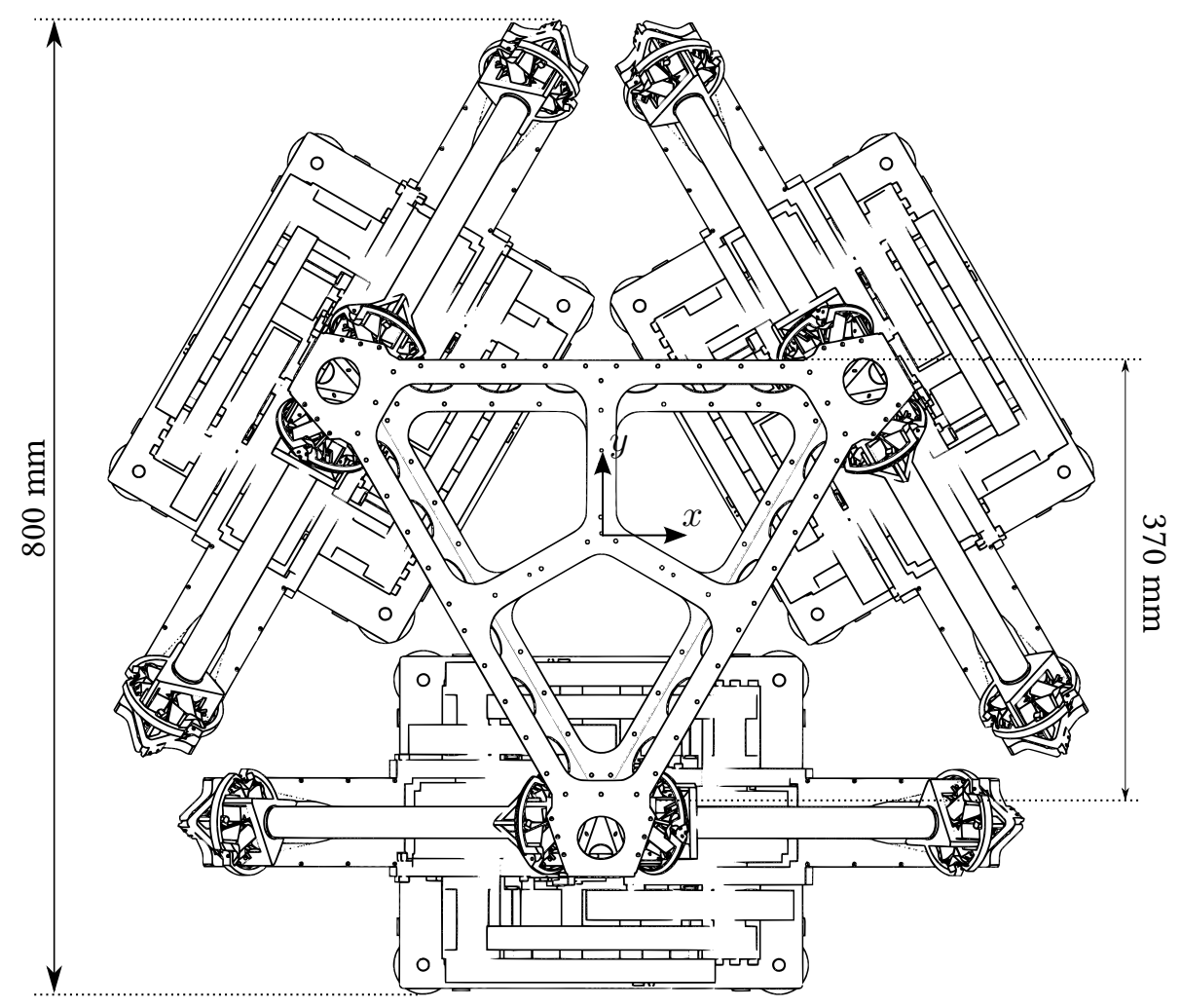

Figure 7.12: Top view of the T-Flex with the main dimensions

vent damage to the spherical joints when they exceed their maximum deflection angle. A micro switch (part 5) is placed parallel to the overload protection to detect a disconnected spherical joint and to shutdown the system.

Furthermore, the lower spherical joint is connected to the second "upper" spherical joint by means of a hollow thin-walled carbon tube (diameter: $28 \mathrm{~mm}$, thickness: $1 \mathrm{~mm}$, length: $250 \mathrm{~mm}$ ). The upper spherical joint provides the connection with the end effector, which is made from aluminum sheet material to provide a lightweight structure. The connection with the folded leafsprings of each upper spherical joint consists of a solid aluminum part (comparable to part 3), which is an integral part of the frame of the end effector. 


\subsection{Detailed system analysis}

\subsubsection{Workspace and eigenfrequencies}

To evaluate the dynamic performance of the system, the eigenfrequencies of the system are analyzed by taking into account the mass and inertia properties of the frame components and the non-flexure parts of the joints. An overview of the mass and inertia properties is provided in appendix 7.11.1. For the dynamic performance of the system, the eigenfrequency that limits the maximum bandwidth of the controller is of direct interest. This eigenfrequency is given by the seventh eigenfrequency of the system with "free" actuators (the first six eigenfrequencies are related to the degrees of freedom of the system). To visualize the frequencies as a function of the end effector location through the workspace, a 2D plane throughout the workspace is selected for displaying this parasitic frequency. The first parasitic frequency as a function of the $z$-position and for equal $x$ - and $y$ position is provided in Fig.7.13 In this figure, the the total displacement in the $x y$-plane is indicated by $d=\sqrt{x^{2}+y^{2}}$ with $x=y$. Furthermore, the boundaries of the workspace of $100 \times 100 \times 100 \mathrm{~mm}^{3}$ are given by the dashed lines. Frequencies outside the workspace, which is determined by the maximum deflection angle of each of the joints, are not displayed. Eigenfrequencies throughout other segments of the workspace, including rotations of the end effector, are provided in appendix 7.11 .2

From the results it can be observed that the required workspace of $100 \times 100 \times$ $100 \mathrm{~mm}^{3}$ is within the range of motion of the hexapod. Furthermore, even larger displacements in the simultaneous directions are allowed, resulting in an actual range of motion of $\pm 105 \mathrm{~mm}$ in $x$-direction, $\pm 100 \mathrm{~mm}$ in $y$-direction and $+95 \mathrm{~mm}$ to $-105 \mathrm{~mm}$ in $z$-direction. The rotational range of motion is \pm 12.5 degrees rotation around the $x$-axis, \pm 11 degrees around the $y$-axis and \pm 15 degrees around the $z$-axis. When instead the internal degree of freedom around the longitudinal axis of the upper arm is not constrained (6- $\overline{\mathrm{R} S S}$ layout), the rotational range of motion is increased to \pm 21 degrees rotation around the $x$ - and $y$-axis and \pm 18 degrees around the $z$-axis. The 6-RुSS layout does suffer from an additional internal degree of freedom, resulting in an extra parasitic eigenfrequency (one for each arm) at approximately $32 \mathrm{~Hz}$, which is nearly constant over the range of motion.

At the center of the workspace the first parasitic frequency is computed at $87 \mathrm{~Hz}$. Within the workspace of $100 \times 100 \times 100 \mathrm{~mm}^{3}$, this frequency drops only slightly to about $74 \mathrm{~Hz}$ at the extrema of this workspace. Over the entire range of motion, the frequency drops further to $60 \mathrm{~Hz}$ at the worst-case location (e.g. at 


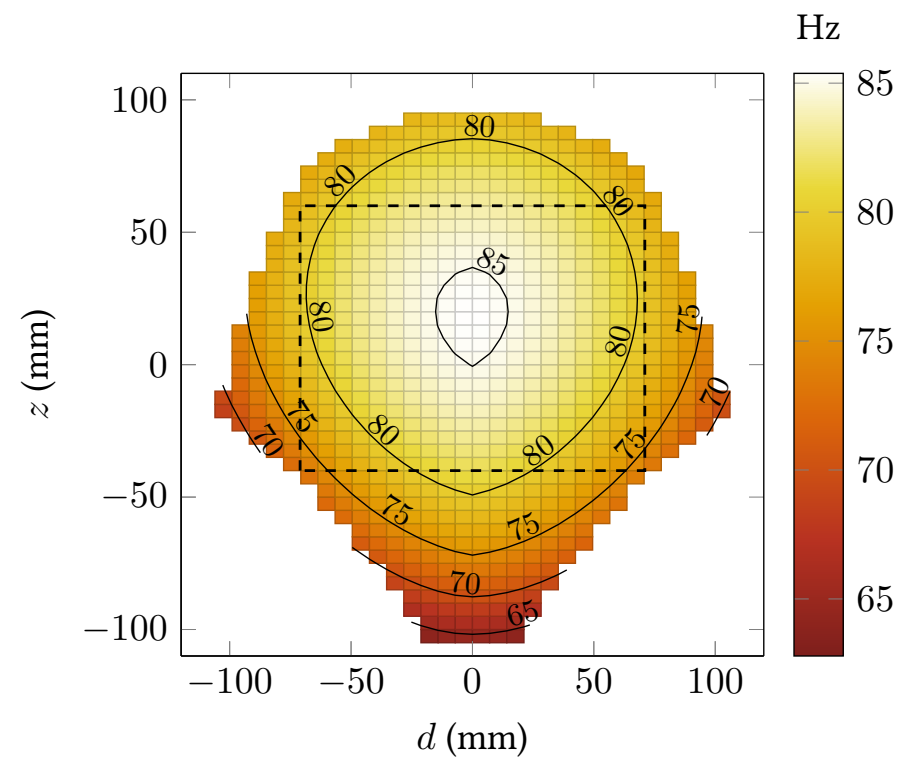

Figure 7.13: First parasitic eigenfrequency as function of the $d$-position $\left(d=\sqrt{x^{2}+y^{2}}\right.$ with $x=y$ ) and the $z$-position of the end effector

$-105 \mathrm{~mm}$ displacement in $z$-direction). A visualization of the modeshape of the critical eigenfrequency at the center of the workspace is provided in Fig.7.14 It consists of a translational motion in the $x$-direction combined with a rotational motion around the $y$-axis. Throughout the workspace, a similar modeshape for the critical parasitic eigenfrequency is found.

It has to be noted that the compliance properties of the frame parts were not included in the computation of the eigenfrequencies. Despite the high rigidity of the frame parts with respect to the flexures, it can be expected that the eigenfrequencies of the actual system are slightly lower, particularly in the neutral position.

\subsubsection{Maximum accelerations}

Given the maximum torque of the actuators, the mass and inertia of the system, and the pose of the mechanism, the maximally achievable accelerations can be computed. Due to reaction forces to counteract gravity and the stiffness of the flexures, the maximum accelerations which can be obtained will be direction dependent (accelerations are either assisted or counteracted by the elastic reaction 


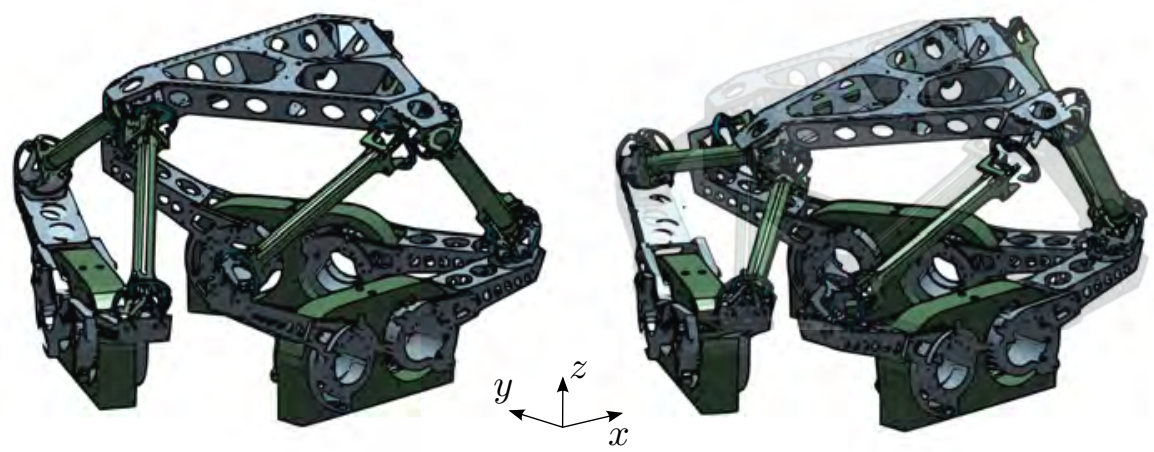

Figure 7.14: Modeshape of the first parasitic frequency at the center of the workspace consisting of a translational motion in the $x$-direction combined with some rotation around the $y$-axis (left: undeflected; right: deflected)

and gravity forces). In this section, the worst-case direction is considered for which the forces are counteracting accelerations.

An example of the maximum accelerations in $z$-direction as function of the $z$ and $d$-position is provided in Fig. 7.15 Accelerations exceeding $120 \mathrm{~m} / \mathrm{s}^{2}$ can be achieved within the ultimate torque range of the actuators over the workspace. The highest accelerations are possible in the center of the workspace, allowing for accelerations up to $180 \mathrm{~m} / \mathrm{s}^{2}$. The maximum accelerations in $x$ and $y$-direction and the rotational accelerations throughout the workspace are provided in 7.11.3 resulting in translational accelerations in the range of 75-150 $\mathrm{m} / \mathrm{s}^{2}$. The maximum rotational accelerations are in the range of $540-850 \mathrm{rad} / \mathrm{s}^{2}$ for rotations around the $x$ - and $y$-axis and 300-550 rad/s $\mathrm{s}^{2}$ for rotations around the $z$-axis

\subsubsection{Maximum load capacity}

Additional payload placed on the end effector of the system will result in an increase in reaction forces. The limiting factor in the maximum force that the end effector can handle is not the maximum torque of the actuators, but the stress in the flexures of the spherical and universal joints. For example, when accepting a $250 \mathrm{MPa}$ increase in Von Mises stress at the worst-case deflection angle of 25 degrees, giving a total stress of about $60 \%$ of the yield stress, a payload up to $100 \mathrm{~N}$ is allowed in the support direction. For smaller deflection angles higher payloads are permitted [68]. 


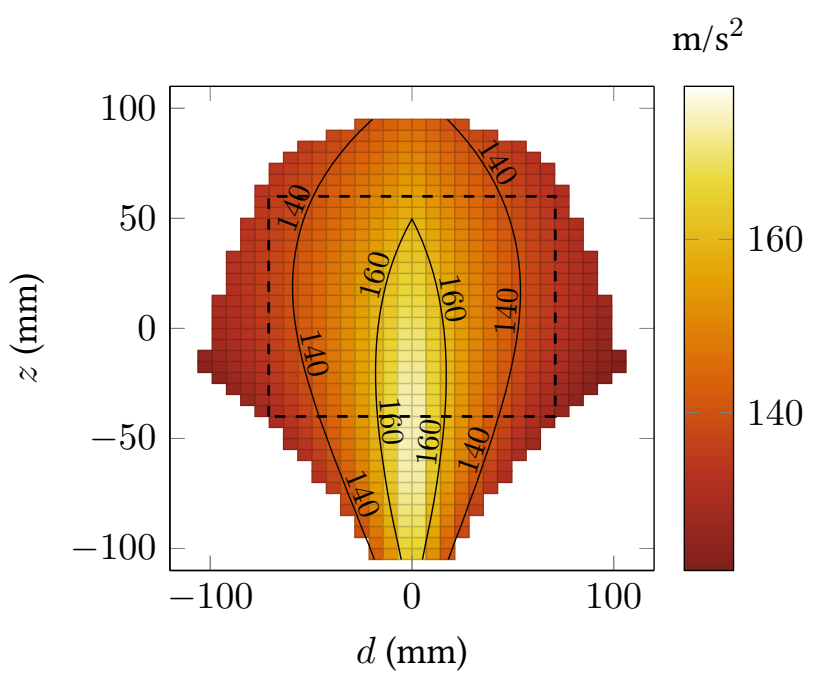

Figure 7.15: Maximum acceleration in $z$-direction as function of the $d$-position $(d=$ $\sqrt{x^{2}+y^{2}}$ with $x=y$ ) and the $z$-position of the end effector

The load on the end effector is distributed over the six legs and the distribution depends on the pose of the mechanism. The worst-case position for static gravity forces is given for the end effector positioned at the bottom of the workspace, which results in a worst-case reaction force in the spherical joints of approximately half of the reaction force exerted on the end effector. Therefore, considering the allowed stress increase of $250 \mathrm{MPa}$, a payload of $200 \mathrm{~N}$ of vertical force is allowed, not including any safety factor.

Note that high payloads in combination with high accelerations also result in increased reaction forces and increased stress levels. Therefore, high payloads also cause reduced maximum accelerations.

\subsubsection{Overview}

A complete overview of the specifications of the T-Flex is listed in Table 7.5 
Table 7.5: Technical data of the T-Flex. Values between parenthesis give the deviating

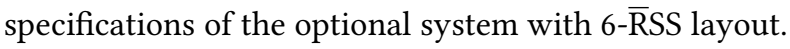

Simultaneous travel range

\begin{tabular}{ll}
\hline \hline Travel range $X$ & $\pm 50 \mathrm{~mm}$ \\
Travel range $Y$ & $\pm 50 \mathrm{~mm}$ \\
Travel range $Z$ & $\pm 50 \mathrm{~mm}$
\end{tabular}

Individual travel range

\begin{tabular}{|c|c|}
\hline Travel range $X$ & $\pm 100 \mathrm{~mm}$ \\
\hline Travel range $Y$ & $\pm 105 \mathrm{~mm}$ \\
\hline Travel range $Z$ & $\pm 100 \mathrm{~mm}$ \\
\hline Travel range $\theta_{X}$ & $\pm 12.5^{\circ}\left( \pm 21.5^{\circ}\right)$ \\
\hline Travel range $\theta_{Y}$ & $\pm 11.5^{\circ}\left( \pm 21.5^{\circ}\right)$ \\
\hline Travel range $\theta_{Z}$ & $\pm 16^{\circ}\left( \pm 18^{\circ}\right)$ \\
\hline Translational workspace & $5.5 \mathrm{dm}^{3}$ \\
\hline \multicolumn{2}{|l|}{ Positioning performance } \\
\hline Max. acceleration $X Y$ & $75-150 \mathrm{~m} / \mathrm{s}^{2}$ \\
\hline Max. acceleration $Z$ & $120-180 \mathrm{~m} / \mathrm{s}^{2}$ \\
\hline Max. acceleration $\theta_{X Y}$ & $540-850 \mathrm{rad} / \mathrm{s}^{2}$ \\
\hline Max. acceleration $\theta_{Z}$ & $300-550 \mathrm{rad} / \mathrm{s}^{2}$ \\
\hline Max. velocity $X Y Z^{*}$ & up to $4 \mathrm{~m} / \mathrm{s}$ \\
\hline \multicolumn{2}{|l|}{ Miscellaneous } \\
\hline Actuator resolution & $13 \mathrm{nrad}$ \\
\hline Actuator torque feedback resolution & $6 \mathrm{Nmm}$ \\
\hline Translational stiffness ${ }^{* *}$ & $250-500 \mathrm{~N} / \mathrm{mm}$ \\
\hline Back-drivable & Yes \\
\hline Footprint (radius) & $0.43 \mathrm{~m}$ \\
\hline Height (excl. electronics) & $0.42 \mathrm{~m}$ \\
\hline Max. vertical payload ${ }^{* * *}$ & $20 \mathrm{~kg}$ \\
\hline Mechanism volume (excl. electronics) & $0.24 \mathrm{~m}^{3}$ \\
\hline
\end{tabular}




\subsection{Prototype design}

\subsubsection{Mechanical design}

A photograph of the completed prototype is provided in Fig. 7.17 For this prototype, the aluminum frame parts of the spherical joints are anodized in red and some of the stationary frame parts at the base are anodized in black. Furthermore, the steel counter masses are painted dark grey. Note that the presented prototype in the figures is of the type 6-RुSS with the additional flexures required to constrain the internal degrees of freedom of the upper arms detached.

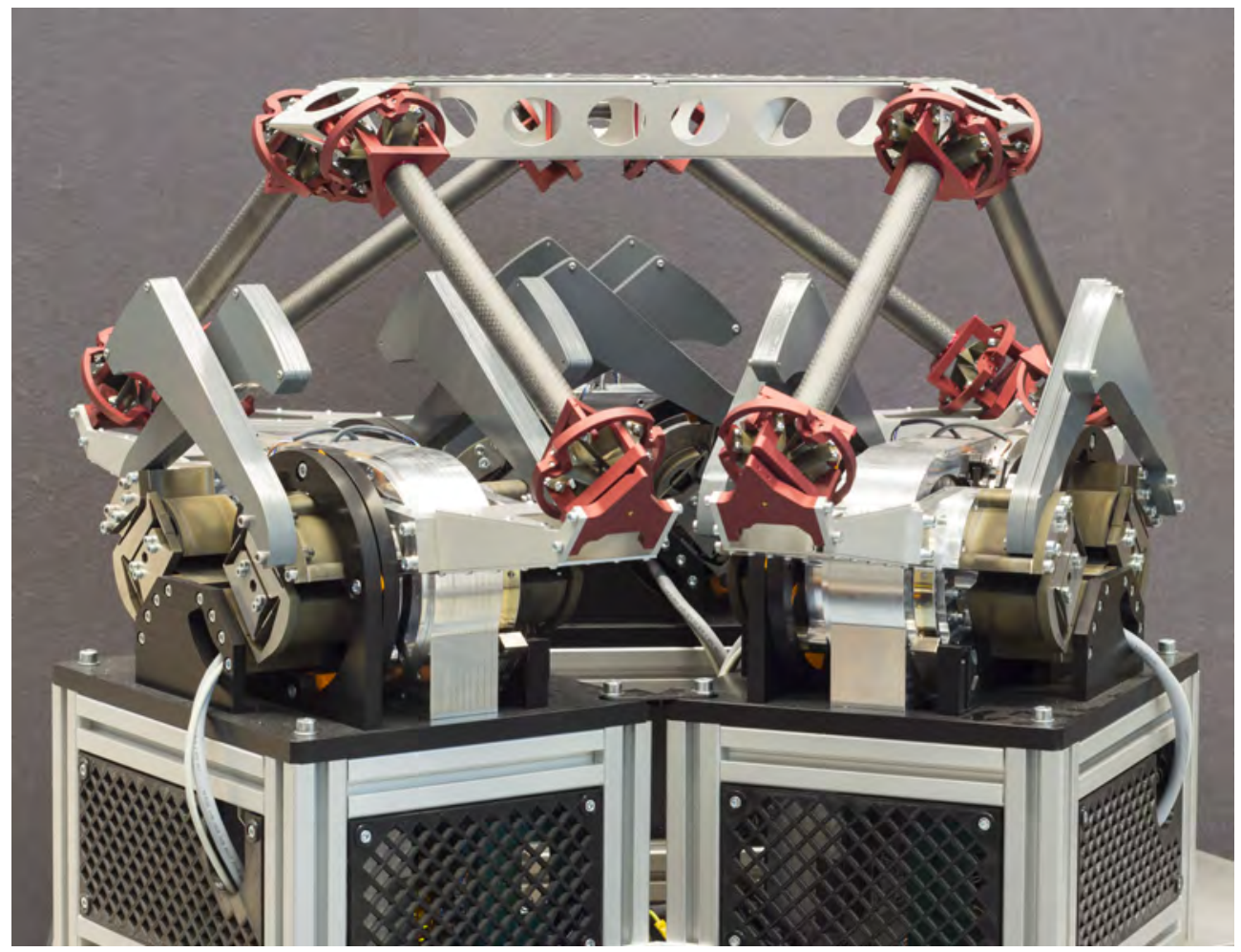

Figure 7.16: Photograph of the T-Flex with the end effector at the center of it's workspace (undeflected) 


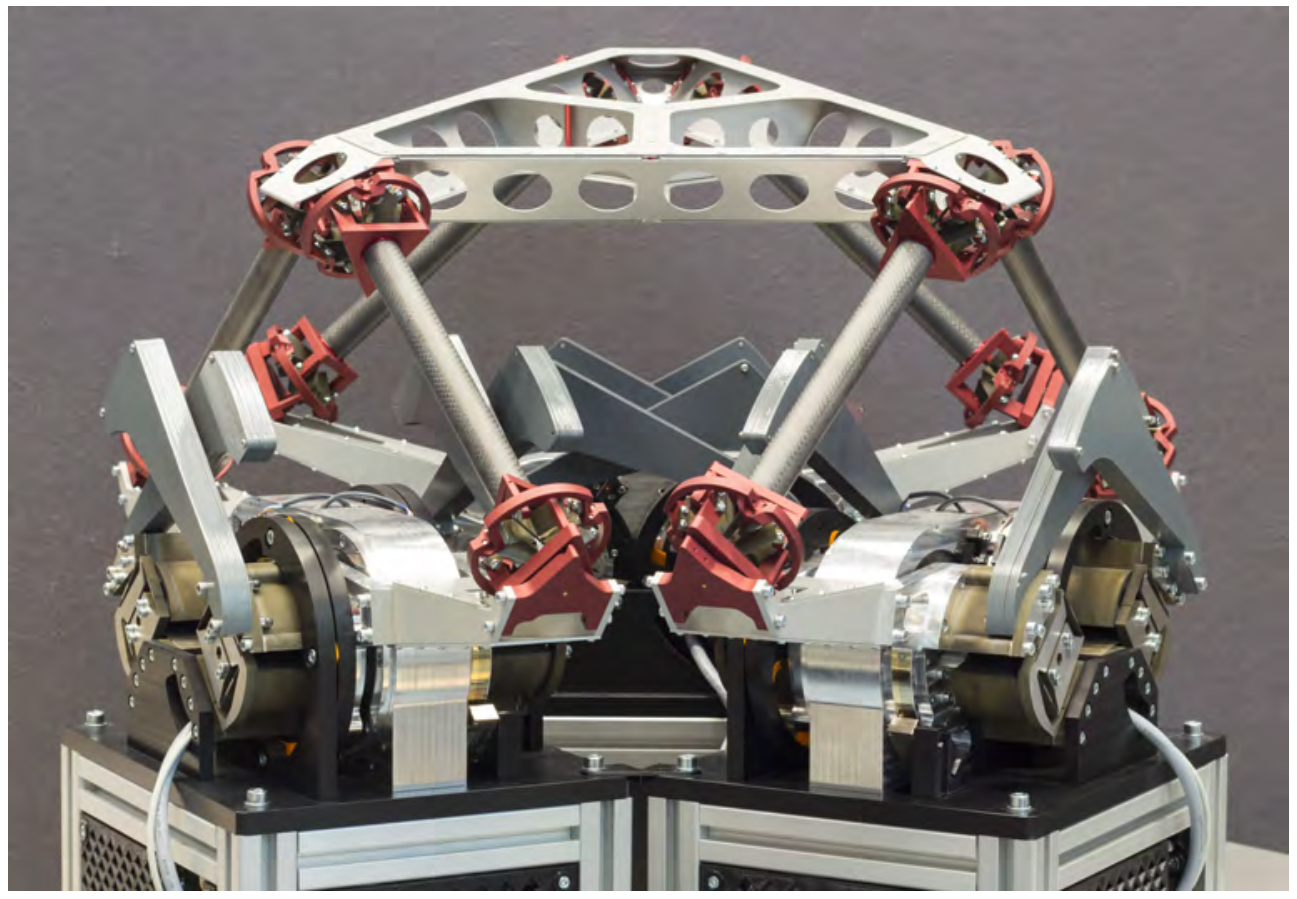

Figure 7.17: Photograph of the T-Flex with the end effector tilted by 10 degrees around the $x$-axis and positioned at $z=30 \mathrm{~mm}$

\subsubsection{System identification}

For evaluating the dynamic performance and designing the controller, first the behavior of the system is identified by evaluating the frequency response function from actuator current to rotations. The frequency response is evaluated by applying a pseudo random binary signal on the provided current (once for each actuator) and measuring the rotation. The results are shown in Fig. 7.18 considering an end effector position in the center of the workspace.

From the results we can see the first natural frequency in the degree of freedom of the actuators at around $2 \mathrm{~Hz}$. Some variation in the first natural frequency can be observed between the actuators, presumably related to initial stress in the flexures induced by minor miss-alignments [59] and variations in the manufacturing process. Furthermore, each of the actuators shows dominant secondorder behavior with a near identical frequency response for each of the actuators. The transfer function of the dominant second order behavior of the plant $(P)$ is 


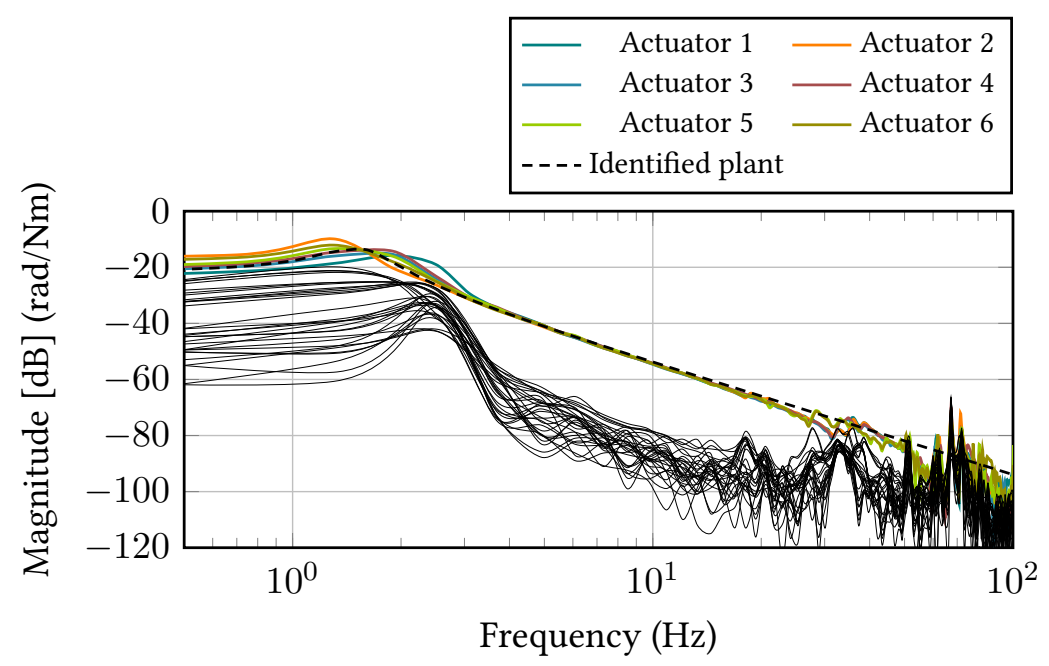

Figure 7.18: Transfer function from actuator current $(\mathrm{mA})$ to rotor position ( $\mathrm{rad})$ at the undeflected state (black lines provide the transfer function from current to rotor position between different actuators).

identified as

$$
P(s)=\frac{1}{m_{e q} s^{2}+d_{e q} s+k_{e q}}=\frac{1}{0.10 s^{2}+0.48 s+12.0}
$$

The first parasitic eigenfrequency of the system can be identified from the strongly excited eigenfrequency just below $70 \mathrm{~Hz}$. Some other resonances with smaller magnitude can be observed at 30-40 Hz, which can be related to the 6 internal

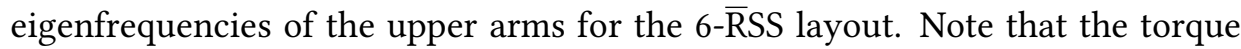
provided by the actuators does not result in significant reaction forces in this underconstrained degree of freedom, producing only low energy input and small excitations. For the same reason, parasitic motion/vibration of this internal degree of freedom does not result in significant error motions at the actuator and end effector.

A measurement of the first parasitic eigenfrequency throughout the workspace is provided in Fig.7.19 Due to more strict safety limits on the actuator positions during the prototyping phase, the outer edges of the workspace are excluded for the experimental results displayed in Fig.7.19. At the center of the workspace, the eigenfrequency is just below $70 \mathrm{~Hz}$ and drops to about $56 \mathrm{~Hz}$ near the edges. This frequency distribution throughout the workspace is in agreement with the simulated eigenfrequencies provided in Fig.7.13 although the frequency values 


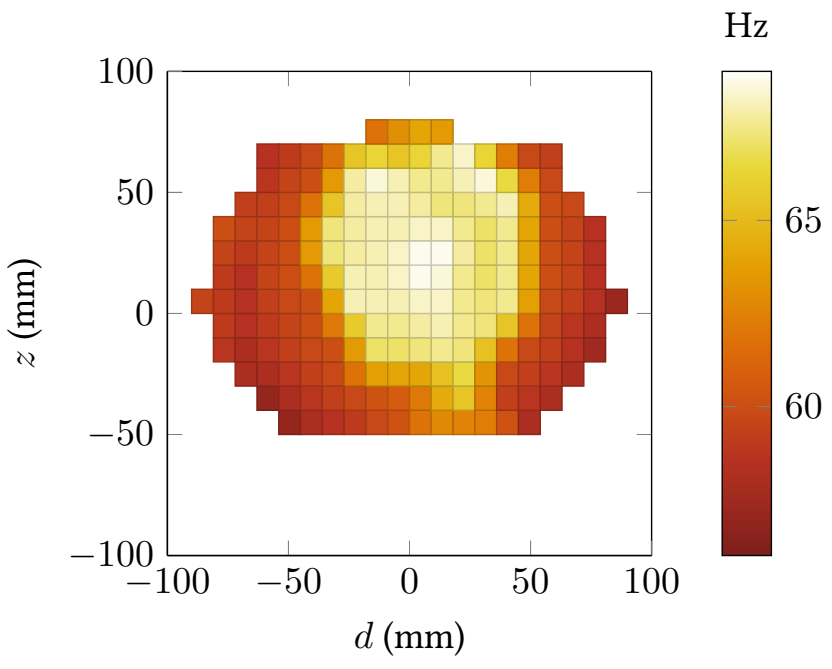

Figure 7.19: Measured first parasitic eigenfrequency as function of the $d$-position $(d=$ $\sqrt{x^{2}+y^{2}}$ with $x=y$ ) and the $z$-position of the end effector

are about $10-20 \%$ lower. This can be attributed to the additional compliance introduced by the frame parts, which have been excluded in the simulations.

\subsubsection{Controller design}

Due to the absence of static friction in the system, accurate positioning is enabled because no limit cycling will occur when using integral control action. On the other hand, the absence of friction also makes the system susceptible to disturbances at standstill. In [70] it has been shown that the main source of positioning error originates from noise on the current signal produced by the motor drivers. In order to reduce this sensitivity and to allow for accurate positioning, a feedback controller is implemented.

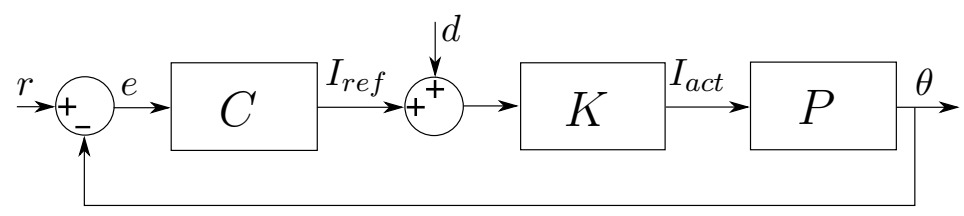

Figure 7.20: Controller overview with: reference signal $(r)$, position error $(e)$, disturbances $(d)$, current reference signal $\left(I_{r e f}\right)$, actual measured current $\left(I_{a c t}\right)$ and actuator angle $(\theta)$ 
An overview of the control scheme is provided in Fig. 7.20 with $C$ the position control loop, $K$ the current control loop and $P$ the plant of the system. Because the cross talk is relatively low in the frequency range up to the first parasitic eigenfrequency, six identical single input single output (SISO) controllers are designed. The goal of the control loops is to minimize the positioning error $e$ given by:

$$
e(s)=\frac{1}{1+C K P} r(s)+\frac{K P}{1+C K P} d(s)
$$

For the transfer function on the left-hand side, we typically aim for a high crossover frequency for good tracking performance combined with sufficient phase margin for a stable system. Below the controller bandwidth $(|C K P| \gg 1)$, the transfer function on the right-hand side can be approximated by $1 / C$. Therefore, a higher crossover frequency provides more disturbance rejection. In the higher frequency range $(|C K P| \ll 1)$, the system can be approximated by $K P$, where additional roll-off provided by the pole of $K$ can provide additional suppression of the higher frequency peaks in $P$. As a result, we aim for a controller with high crossover frequency combined with a stable closed loop over the entire workspace, with additional roll-off in the current control loop to suppress high frequency peaks of the plant. Note that the balance masses attached to the rotor provide a reduction in the magnitude of $P$.

For the current controller $K$, the built-in current controller of the motor driver is used, which is structured as a low-pass filter. For the position control loop a PID controller is used. The controller parameters are

$$
\begin{aligned}
& C=k_{p} \frac{\left(\tau_{i} s+1\right)\left(\tau_{z} s+1\right)}{\tau_{i} s\left(\tau_{p} s+1\right)} \\
& K=\frac{1}{\tau_{c} s+1}
\end{aligned}
$$

with

$$
\begin{array}{rlrl}
k_{p} & =382.9 & \tau_{p}=0.0014 \\
\tau_{i} & =0.0495 & & \tau_{c}=0.0014 \\
\tau_{z} & =0.0495 & &
\end{array}
$$

which provides a crossover frequency at $20 \mathrm{~Hz}$ and a phase margin of 44 degrees. The controller has been tuned for maximum performance and stability over the entire workspace. However, it should be noted that the effect of end effector payload has been neglected. As the focus of this paper is on the structural optimization, mechanical design and experimental validation of this system, the controller design is not described in more detail. 


\subsection{Experimental validation}

\subsubsection{The standstill performance}

As the positioning performance of the system is strongly affected by current noise produced by the motor drivers, the influence of current noise on the actuator and end effector position is evaluated. To measure the position of the end effector, three capacitive displacement sensors (Lion Precision C6, resolution $<1 \mathrm{~nm}$ ) are placed in a circular pattern in an external frame measuring the $z$-position of the end effector. By combining the sensor data of the three sensors, the $z$-position and rotations around the $x$ - and $y$-axis can be evaluated simultaneously.

To analyze the influence of current noise on maintaining a stationary end effector position as a function of frequency, the results in this section are provided as the square root of the cumulative sum of the power density multiplied with the spectral resolution. According to Parseval's theorem, the complete sum provides the total RMS error according to

$$
e_{R M S}=\sqrt{\sum S \Delta f}
$$

with $S$ the power spectral density of the time-signal and $\Delta f$ the spectral resolution.

The resulting cumulative positioning error of the actuators, measured at the actuators including feedback control for a stationary target position, is provided in Fig. 7.21. These results show an error between 1.5-3.5 $\mu$ rad RMS. The disturbance at low frequency $(<20 \mathrm{~Hz})$ is in part rejected by the feedback controller. Furthermore, a large contribution is observed at $69 \mathrm{~Hz}$, which is caused by the high amplification of the plant at the parasitic eigenfrequency.

The measured position at the end effector is provided in Fig. 7.22 The results show a $0.34 \mu \mathrm{m}$ RMS error in the $z$-direction and a 4-5 $\mu \mathrm{rad}$ RMS error in the rotational $x$ - and $y$-direction. Note that the contribution at the parasitic eigenfrequency mainly affects the error in the $\theta_{r x}$ and $\theta_{r y}$ direction, which corresponds to the expected modeshape as visualized in Fig.7.14 It has to be pointed out that

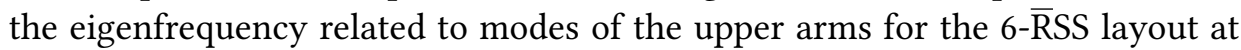
$30-40 \mathrm{~Hz}$ does not mitigate precision at the end effector. 

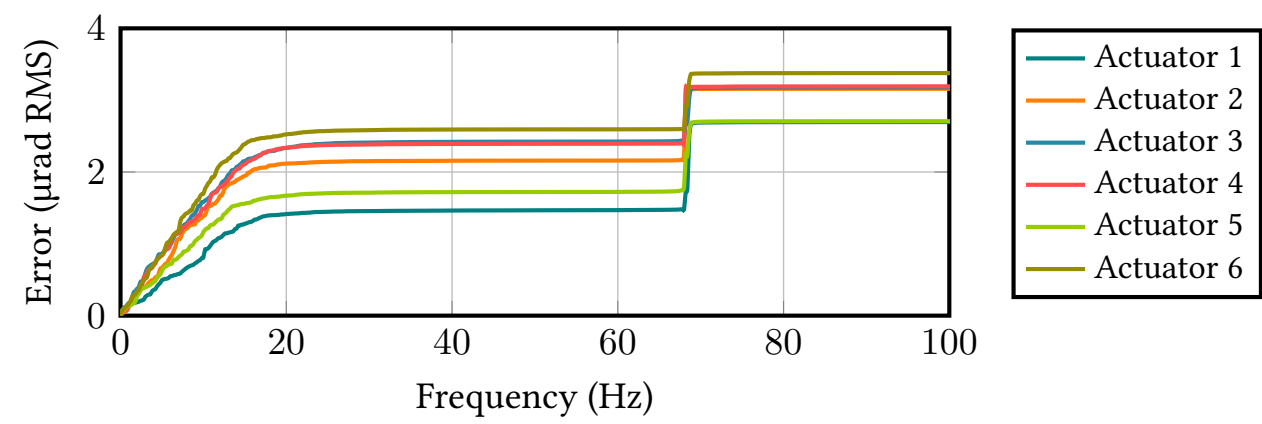

Figure 7.21: Cumulative RMS error of the actuator position including feedback control

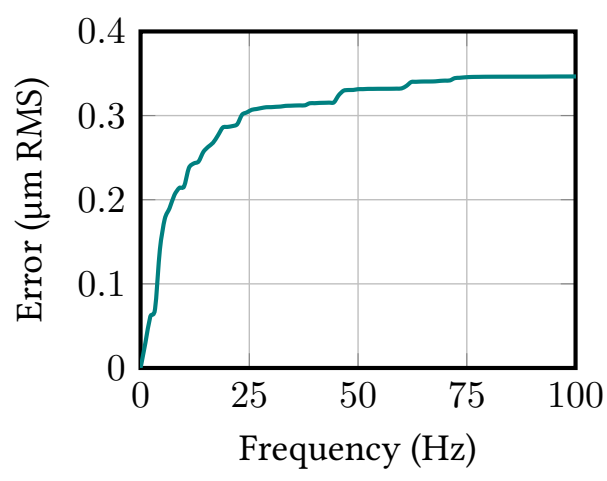

(a)

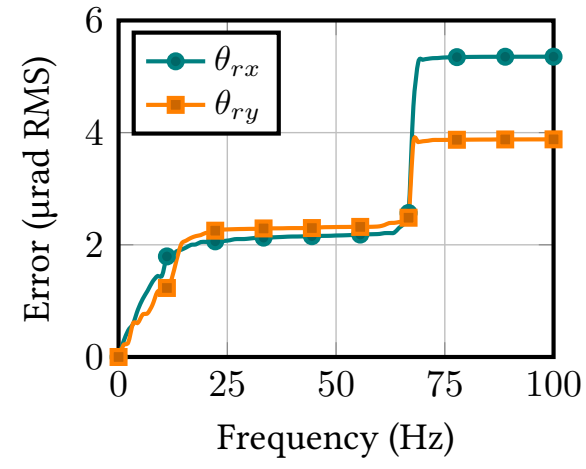

(b)

Figure 7.22: Cumulative RMS error with active feedback control measured at the end effector in (a) the translational $z$-direction and (b) the rotational $x$ - and $y$-direction

\subsubsection{Repeatability}

For evaluating the repeatability of the system, the capacitive displacement sensors described earlier are used to measure the $z$-position of the end effector. For this test, the end effector is moved repeatedly in $z$-direction with a bi-directional displacement of $\pm 100 \mu \mathrm{m}$ at an interval of 2.5 seconds combined with a large displacement of $+50 \mathrm{~mm}$ at an interval of 17.5 seconds. The measurement results, provided in Fig. 7.23, show highly repeatable behavior well bellow the magnitude of the position fluctuations caused by disturbances. It can be observed that the repeatability is independent of the direction of movement, which would be typical for a system which observes friction, play or hysteresis.

Additionally, the averaged long-term end effector position for a period of $400 \mathrm{sec}-$ 


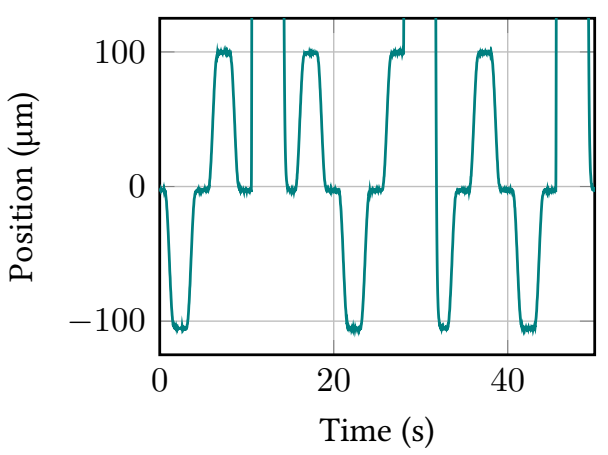

(a)

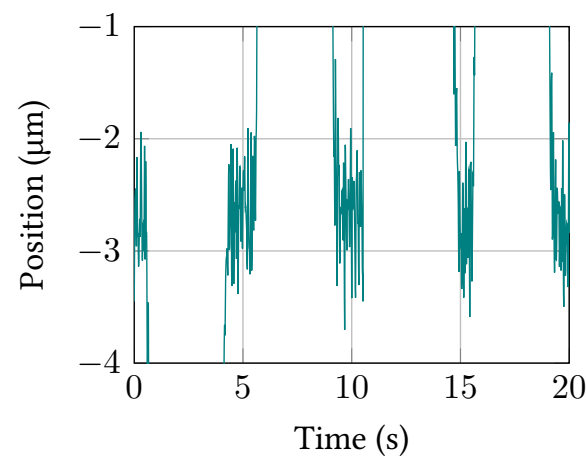

(b)

Figure 7.23: (a) Repeatability measurement for the $z$-position of the end effector and (b) a zoomed section of the repeatability measurement

onds is provided in Fig.7.24, showing fluctuations of $\pm 0.25 \mu \mathrm{m}$ of the mean end effector position. For reference, the rotation of one of the actuators is provided in Fig. 7.25 showing a constant rotation angle. The measured values are in range of expectation considering dimensional changes due to temperature variations in the environment and heat input from the actuators and electronics. For example, when considering the linear temperature expansion coefficient for aluminum and steel of $10-25 \mu \mathrm{m} /\left(\mathrm{m}^{\circ} \mathrm{C}\right)$ and a roughly estimated linear dimension of $0.1 \mathrm{~m}$, a $0.1{ }^{\circ} \mathrm{C}$ temperature change can already result in $0.25 \mu \mathrm{m}$ change in length. Note that the base for the actuators have been equipped with cooling channels which allows for active cooling if deemed necessary. For the measurements in this section, no active cooling is employed. 


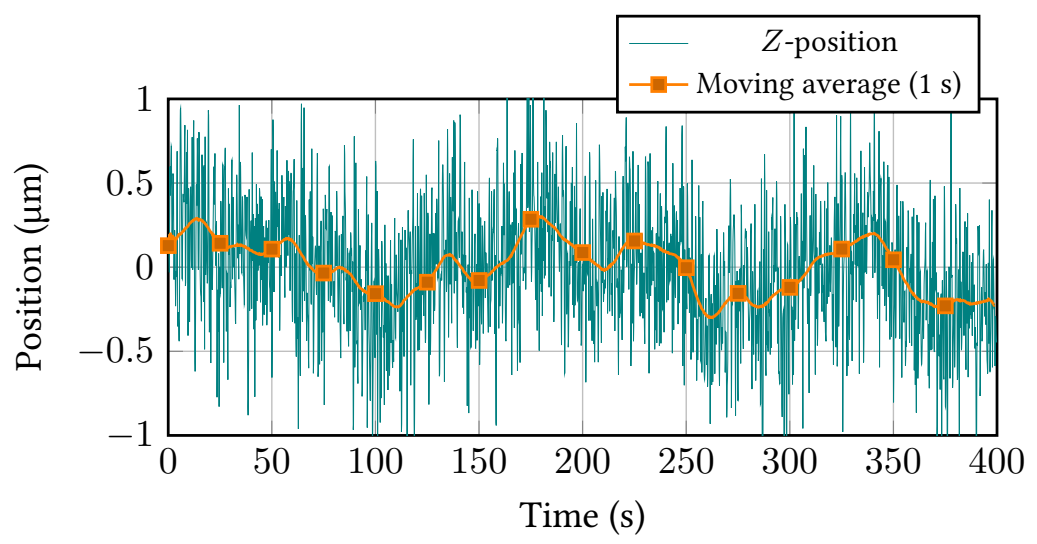

Figure 7.24: End effector $z$-position measured with a stationary target position

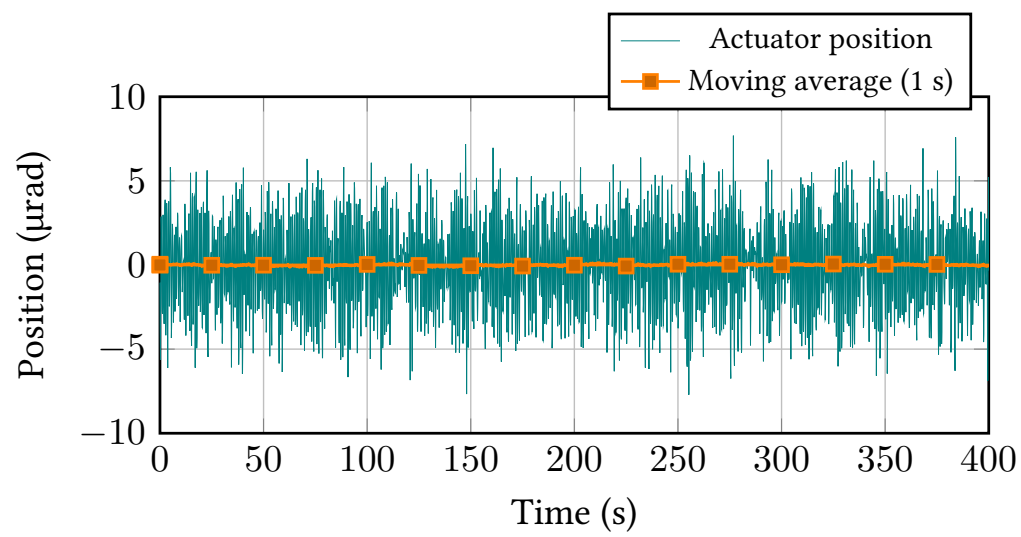

Figure 7.25: Actuator position of actuator 1 with a stationary target position

\subsection{Discussion}

Assuming a fixed relative magnitude of the disturbances in the provided current with respect to the ultimate current, the standstill performance is directly affected by the motor constant of the actuators combined with the inertia of the system. Therefore, the influence of input disturbances can be adjusted in order to meet specified criteria. For example, motor torque (the motor constant) can be sacrificed to reduce sensitivity to disturbances. However, the designer will always face a trade-off between the standstill performance and the maximum torque which can be delivered, directly affecting the maximum accelerations. Moreover, as the standstill performance is currently restricted by limitations of 
the electronics, it can be improved by using a better electronics design. The currently used industrial class PWM-based motor drivers provide a peak current to noise ratio $<1000$, which can be considered typical for a motor driver. To further improve this ratio, an amplifier with higher performance is a necessity, e.g. an linear amplifier. As this is expensive especially considering the high power requirements for the actuators, and as the mechanical design is the main focus of this research, this has not been improved on.

Furthermore, the position stability of the end effector position appears to be limited, possibly due to temperature variations in the system. It is expected that a homogeneous temperature change of only $0.1^{\circ} \mathrm{C}$ can already cause dimensional changes up to $0.25 \mu \mathrm{m}$. An additional source of disturbances can be found in the small, but not negligible, amount of hysteresis in flexures. For flexure mechanisms with clamped flexures, the magnitude of hysteresis has been measured in the order of magnitude of $0.1 \%$ [103]. For flexure mechanisms with monolithic flexures, which is the case for the T-Flex, the magnitude of hysteresis can be expected to be even smaller. This means that the elastic reaction forces in the degrees of freedom of the joints can vary (e.g. 1/1.000 Nm variation). Combined with the stiffness of the system, this can result in deformations in the order of magnitude of tenths of nanometers.

A commonly used approach to improve positioning performance in high precision systems is the use of an additional feedback loop with respect to the end effector position [78]. With this additional feedback, low frequent disturbances caused by hysteresis and temperature changes can be easily compensated for. Alternatively, additional effort can be put in stabilizing the temperature of both the environment and the system [16].

\subsection{Conclusion}

A large range of motion flexure-based hexapod has been designed which allows for a $\pm 100 \mathrm{~mm}$ of translational and more than \pm 10 degrees of rotational range of motion combined with a sub-micron repeatability. The design has been optimized by considering each elastic joint as an isolated component, lumping the nonlinear compliance behavior of the flexure-based joints. Combined with some practical symmetry relations, a fast model with a small set of design parameters has been created for efficient optimization.

The optimization results have led to a fully flexure-based hexapod design, the T-Flex, which provides a translational end effector stiffness between 250 and 
$500 \mathrm{~N} / \mathrm{mm}$ throughout the workspace. Furthermore, the first parasitic eigenfrequency at $56-70 \mathrm{~Hz}$ throughout the workspace allows for a $20 \mathrm{~Hz}$ control bandwidth. The specifically designed elastically suspended actuators permit high payload, high actuation torque and back-drive-ability allowing for torque feedback capabilities. Due to the high torque actuators, the system is able to achieve high accelerations (up to $18 \mathrm{~g}$ ), unprecedented travel speed (exceeding $1 \mathrm{~m} / \mathrm{s}$ ) and a large vertical payload of $20 \mathrm{~kg}$. This is a large improvement over existing high-precision parallel manipulators, since the travel range is up to an order of magnitude larger and the maximum travel speed has been increased by two orders of magnitude.

Since the design is completely free from play, friction and cable disturbances, the repeatability of the system is only limited by the electronics. This results in disturbances on the provided current signal to the actuators, leading to $0.35 \mu \mathrm{m}$ RMS position noise on the end effector. Further increasing the precision and the implementation of feed forward control strategies to improve tracking accuracy will be subject of future research. 


\subsection{Appendices}

\subsubsection{Mass and inertia properties}

A schematic overview of the locations of the centers of mass of the frame parts and the orientation of the axes used to define the inertia properties is provided in Fig.7.26. The center of mass of the end effector $\left(I^{e}\right)$ and the upper arms $\left(I^{u}\right)$ are positioned in the center of the parts. The center of mass of the lower arms $\left(I^{l}\right)$ are located at a vertical distance of $56 \mathrm{~mm}$ and at a horizontal distance of $23 \mathrm{~mm}$ with respect to the rotation axis of the revolute joint (as indicated in Fig. 7.26).

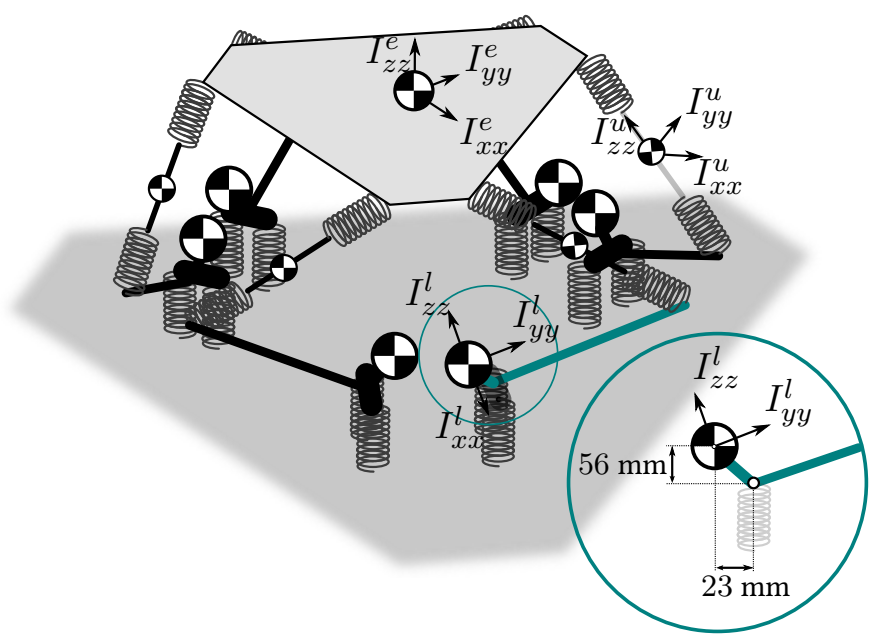

Figure 7.26: Assignment of the mass properties for the T-Flex (dimensions not to scale)

Table 7.6: Approximate mass and inertia properties

\begin{tabular}{lllll} 
Part & $m$ & $I_{x x}$ & $I_{y y}$ & $I_{z z}$ \\
\hline \hline Lower arm $(l)$ & $6.83 \mathrm{~kg}$ & $0.031 \mathrm{kgm}^{2}$ & $0.025 \mathrm{kgm}^{2}$ & $0.038 \mathrm{kgm}^{2}$ \\
Upper arm $(u)$ & $0.27 \mathrm{~kg}$ & $0.007 \mathrm{kgm}^{2}$ & $0.007 \mathrm{kgm}^{2}$ & $0.0002 \mathrm{kgm}^{2}$ \\
End effector $(e)$ & $0.91 \mathrm{~kg}$ & $0.019 \mathrm{kgm}^{2}$ & $0.019 \mathrm{kgm}^{2}$ & $0.038 \mathrm{kgm}^{2}$ \\
\hline
\end{tabular}

For the end effector, $I_{z z}^{e}$ provides the inertia with respect to the vertical axis and $I_{x x}^{e} \approx I_{y y}^{e}$ the inertia in horizontal $x y$-plane. For the upper arm, $I_{z z}^{u}$ gives the inertia around the longitudinal axis, and $I_{x x}^{u}=I_{y y}^{u}$ the inertia perpendicular to this axis. At last, $I_{x x}^{l}$ gives the inertia along the rotation axis of the revolute base joint, $I_{y y}^{l}$ the inertia along the axis intersecting the revolute joint and the lower 
spherical joint (the axis aligned with the lower arm), and $I_{z z}^{l}$ the inertia around the remaining axis. An overview of the approximate mass and inertia properties are provided in Table 7.6 which is retrieved from CAD data.

\subsubsection{First parasitic eigenfrequency}

Fig. 7.27 provides the first parasitic frequency as function of the end effector position throughout the workspace for positive $x$ - and $y$-positions. In order to visualize the frequency as function of 3D motion, the values are provided in three planes with $x=0, y=0$ and $z=0$. Furthermore, a diagonal plane is provided for which $x=y$ which includes two extrema of the $100 \times 100 \times$ $100 \mathrm{~mm}$ workspace. Fig. 7.28 provides the first parasitic frequency as function of rotational end effector motion with the rotation axis in the $x y$-, $y z$ - and the $x z$ plane. Fig.7.28a provides the results for the 6-奋US hexapod layout and Fig. $7.28 \mathrm{~b}$ for the $6-\overline{\mathrm{RSS}}$ layout.

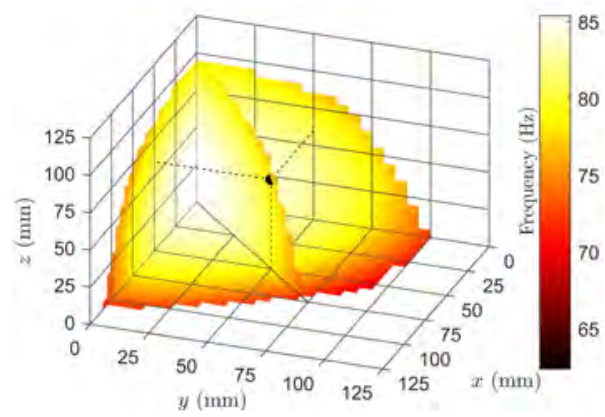

(a)

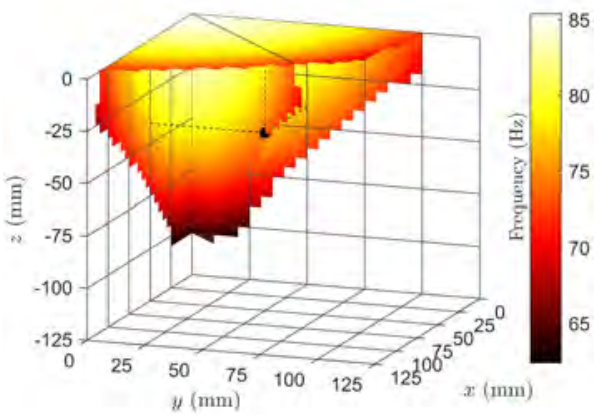

(b)

Figure 7.27: First parasitic eigenfrequency as function of translational motion through the workspace 


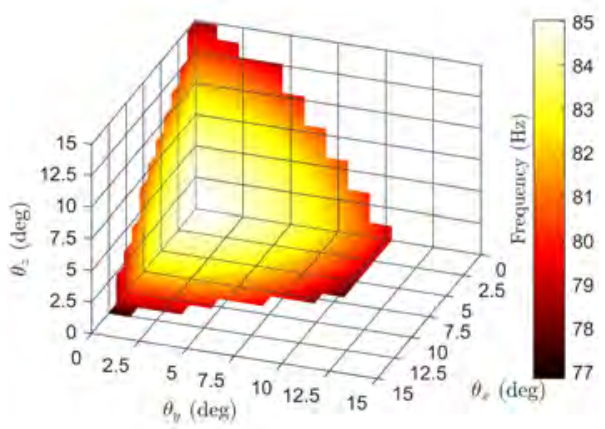

(a)

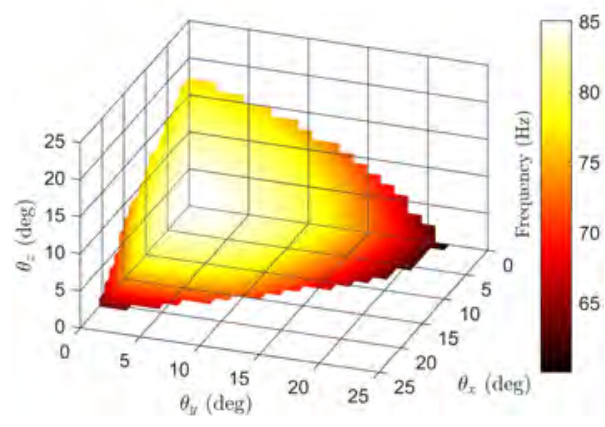

(b)

Figure 7.28: First parasitic eigenfrequency for end effector rotations for the (a) $6-\bar{R} U S$ and the (b) 6- $\overline{\mathrm{R}} S \mathrm{~S}$ hexapod layout

\subsubsection{Maximum accelerations}

Fig. 7.29 provides the maximum translational accelerations as function of the end effector position (evaluated for end effector positions with $x=y$ ). On the horizontal axis the total displacement in the $x y$-plane is indicated according to $d=\sqrt{x^{2}+y^{2}}$ (with $x=y$ ). Maximum rotational accelerations around the $x$-, $y$ - and $z$-axis are provided in Fig. 7.30 


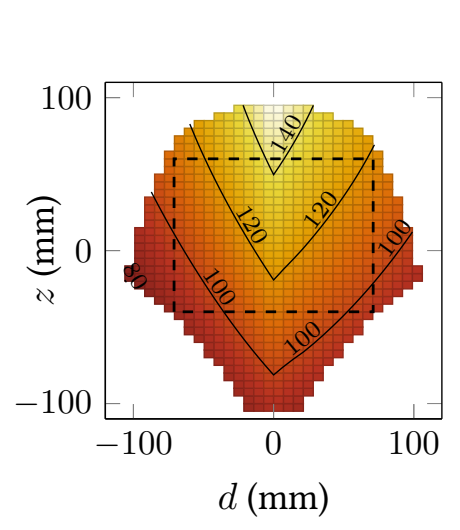

$\mathrm{m} / \mathrm{s}^{2}$

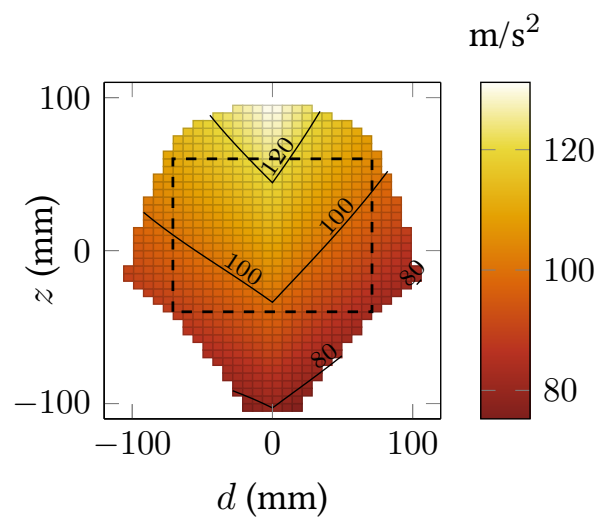

(a) Maximum acceleration in $x$ direction

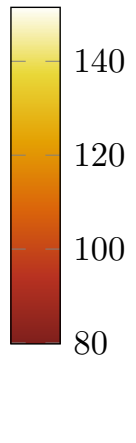

(b) Maximum acceleration in $y$ direction

$\mathrm{m} / \mathrm{s}^{2}$
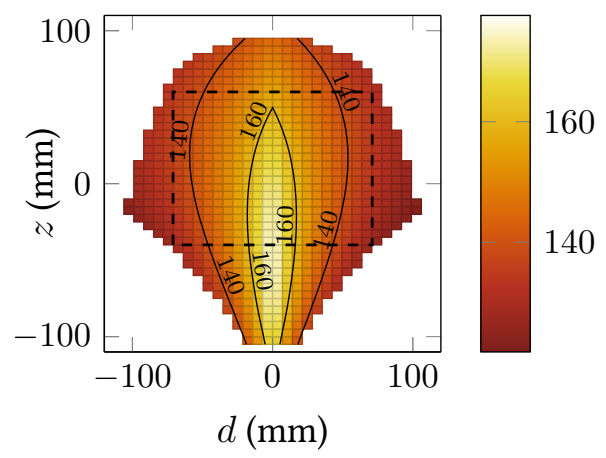

(c) Maximum acceleration in $z$ direction

Figure 7.29: Maximum translational accelerations through the workspace with $d=$ $\sqrt{x^{2}+y^{2}}$ and $x=y$ 
$\mathrm{rad} / \mathrm{s}^{2}$

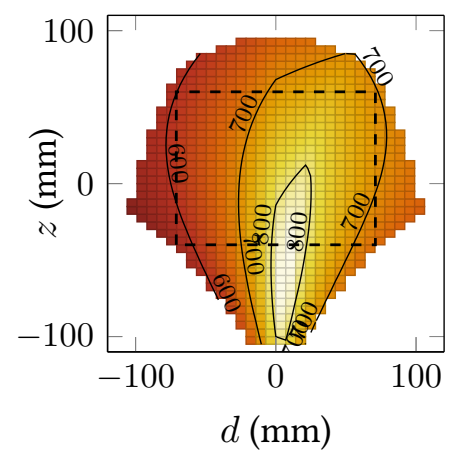

(a) Maximum rotational acceleration around the $x$-axis

$$
\mathrm{rad} / \mathrm{s}^{2}
$$

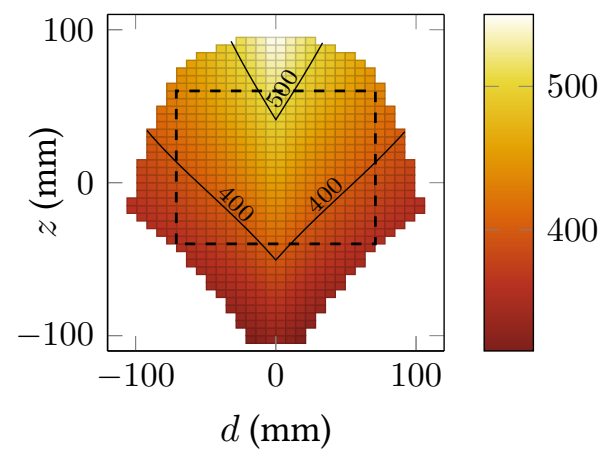

(c) Maximum rotational acceleration around the $z$-axis

Figure 7.30: Maximum rotational accelerations through the workspace with $d=$ $\sqrt{x^{2}+y^{2}}$ and $x=y$ 



\section{Conclusions and recommendations}

The overarching goal of this thesis is to develop flexure-based mechanisms with a large range of motion combined with high support stiffness and load capacity to enable new precision applications. Three sub-goals were formulated in the introduction; 1) the development of an efficient optimization strategy for complex large stroke flexure mechanisms, 2) the development of large stroke flexure-based joints and 3) the design of a flexure-based six degree of freedom manipulator with large range of motion. This chapter provides the outcome of this research and reflects on the research results and objectives.

\subsection{Conclusions}

With respect to the first objective, "the development of an efficient optimization strategy for large stroke flexure mechanisms", we can conclude that this objective has been achieved by choosing a shape optimization algorithm combined with a well-chosen initial layout for the considered flexure mechanisms based on the theory of degrees of constraint and freedom. The efficiency of this method is enhanced by using an adaptive objective function combined with constraint interpolation in order to improve convergence. The applicability of the developed optimization strategy is demonstrated by the outcome for the second research objective, "the development of large stroke flexure-based joints", which has resulted in new and improved designs for large stroke revolute, universal and spherical flexure-based joints. 
For the revolute flexure joint, a design strategy is demonstrated which provides improved hinge designs for a broad selection of load cases, enabled by a buildingblock based layout variation strategy (Chapter 2). This resulted in a flexure-based revolute joint that is able to reach a stroke of \pm 45 degrees while maintaining near-constant support stiffness over the full range of motion. At maximum deflection, this provides an increase in support stiffness of almost two orders of magnitude compared to the customary cross-spring pivot.

The optimization of universal and spherical joints has proven to be more complex due to their spatial topology, resulting in collision between the flexures when deflected. To overcome this, a collision detection method was developed to enable optimization of the flexure geometry while avoiding collision (Chapter 3). By discretizing the shape of the leafsprings, the computational cost for evaluating collision can be kept small, not hampering the optimization.

The collision detection method helped to enable the optimization of a new layout for a spherical joint, consisting of a serial parallel-stacked arrangement of folded leafsprings. An optimized design provided a tip-tilt motion of \pm 30 degrees and a pan motion of \pm 10 degrees (Chapter 4). By effectively stacking two spherical joints in series with a coinciding instant center of rotation, a large range of motion and high support stiffness are obtained. Based on a similar design concept as the spherical joint, a flexure-based universal joint is presented, allowing for a large tip-tilt motion of \pm 25 degrees combined with high support stiffness (Chapter 5).

The last research objective, "the design of a six degree-of-freedom parallel manipulator with large range of motion", has been successfully accomplished by using the improved designs for the universal and spherical flexure joint and an adapted design for the existing butterfly hinge (Chapter 6 and 7). The designed hexapod can provide sub-micron repeatability and enables a travel range of $\pm 100 \mathrm{~mm}$ in each translational direction. Furthermore, a rotational range of motion above \pm 10 degrees in each rotational direction, accelerations beyond $100 \mathrm{~m} / \mathrm{s}^{2}$ (no payload) and velocities exceeding $1 \mathrm{~m} / \mathrm{s}$ are obtained. This exceeds the performance characteristics of other state-of-the-art flexure-based hexapod systems in many aspects.

Overall, it has been shown that large stroke flexure-based joints are possible which can provide high support stiffness even at large deflection angles. This allows for increased stiffness and range of motion for flexure-based systems and enables the employment of flexure-based joints for new applications which where not feasible before. 


\subsection{Discussion and Recommendations}

In this thesis it has been shown that the performance of large stroke flexurebased joints can be improved by utilizing a shape optimization strategy and a dedicated joint topology. However, for deriving a good initial joint topology, only a limited number of building blocks or joint configurations were considered, restricting the design freedom. To further extend the reach of the optimization procedure, the use of more building blocks and more freedom in topology is recommended. This could be achieved by using degrees of freedom and constraints for typical flexure elements and systematically combining them in parallel and series. This strategy was already proven to be effective for creating new building block topologies as illustrated in Chapter 2 Including this additional "design freedom" in the optimization procedure can potentially lead to new improved flexure hinge designs.

Furthermore, the main focus of this thesis has been on the optimization of the flexure geometries for maximizing support stiffness and load capacity in the load-carrying directions. However, some applications can have other demands which require a different joint design. This is also encountered for the design of the flexure-based actuator suspension of the T-Flex (Chapter 6), which demanded a low parasitic motion of the rotor which is not incorporated in the layout variation strategy and building blocks discussed in Chapter 2 In order to meet the requirements, the cartwheel and butterfly hinge layout are considered instead, which are not within the design space of the presented layout variation strategy. To improve the applicability of the optimization method, other typically encountered requirements for flexure-based mechanisms could be taken into account in the optimization procedure. However, for the specific case of minimizing parasitic motion, it has to be noted that parasitic motion depends strongly on the chosen flexure topology while the geometry of the flexures has only a limited influence. Therefore, in order to provide an efficient solution, this likely requires an automated topology variation strategy as mentioned in the previous paragraph.

For maintaining high support stiffness even at large deflection angles, flexurebased joint designs typically become increasingly complex. As a result, the joint assemblies consist of more components and possibly more advanced manufacturing techniques are required (e.g. wire EDM or additive manufacturing). Consequently, the designer will have to face a trade-off between complexity, cost and performance. Furthermore, when considering large range of motion applications, the geometric interference between the flexures and frame parts starts to play a crucial role. This can be partially taken into account in the optimization 
procedure as illustrated in Chapter 3 However, the range of motion is often inherently limited by the chosen flexure topology due to collision of frame parts or the flexures, limiting the maximum range of motion which can be obtained. For example, the spherical flexure joint design provided in Chapter 4 inhibits a range of motion well beyond 30 degrees of tip-tilt. A possible solution to further extend the range of motion can be provided by connecting multiple flexure joints in series. However, the resulting unintended internal degrees of freedom can be challenging to deal with.

Finally, as the design of flexure-based mechanisms involves multiple technical disciplines such as electronics, control and mechanical engineering, they cannot be considered independently due to their strong interaction. In the design process for the T-Flex provided in Chapter 7, the mechanics, electronics and controls were designed in parallel with iterative checks on their interaction. Consequently, the design was adapted based on the requirements and constraints imposed by each of the different disciplines. This way the various domains have been properly interfaced and the desired mechatronic system-level performance requirements have been realized. However, with a clear application in mind, the system-level performance can be further improved by an integral optimization strategy including each of the individual disciplines. 


\section{Bibliography}

[1] E. Abbe. Messapparate für Physiker. Zeitschrift für Instrumentenkunde, 10(12):446-447, 1890.

[2] G. Allaire, F. de Gournay, F. Jouve, and A.-M. Toader. Structural optimization using topological and shape sensitivity via a level set method. Control and cybernetics, 34(1):59-80, 2005.

[3] G. Allaire, F. Jouve, and A.-M. Toader. Structural optimization using sensitivity analysis and a level-set method. fournal of Computational Physics, 194(1):363-393, 2004.

[4] S. Awtar, A. H. Slocum, and E. Sevincer. Characteristics of Beam-Based Flexure Modules. Fournal of Mechanical Design, 129(6):625-639, 2007.

[5] J. P. Bacher, S. Bottinelli, J.-M. Breguet, and R. Clavel. Delta 3 : design and control of a flexure hinge mechanism. Microrobotics and Microassembly III, 4568:135-142, 2001.

[6] M. P. Bendsøe and N. Kikuchi. Generating Optimal Topologies in Structural Design Using a Homogenization Method. Computer Methods in Applied Mechanics and Engineering, 71(2):197-224, 1988.

[7] C. Brecher, N. Pyschny, and J. Behrens. Flexure-based 6-axis alignment module for automated laser assembly. In International Precision Assembly Seminar, pages 159-166. Springer, 2010.

[8] D. M. Brouwer, J. P. Meijaard, and J. B. Jonker. Large deflection stiffness analysis of parallel prismatic leaf-spring flexures. Precision Engineering, 37(3):505-521, 2013.

[9] D. M. Brouwer, A. Otten, J. B. C. Engelen, B. Krijnen, and H. M. J. R. Soemers. Long-range Elastic Guidance Mechanisms for Electrostatic Combdrive Actuators. In Proceedings of the Euspen Intenational Conference, 2010.

[10] T. Buhl, C. B. W. Pedersen, and O. Sigmund. Stiffness Design of Geometrically Nonlinear Structures Using Topology Optimization. Structural and Multidisciplinary Optimization, 19(2):93-104, 2000.

[11] A. N. Christiansen, J. A. Bærentzen, M. Nobel-Jørgensen, N. Aage, and 
O. Sigmund. Combined shape and topology optimization of 3D structures. Computers \& Graphics, 46:25-35, 2015.

[12] F. Cosandier, S. Henein, M. Richard, and L. Rubbert. The Art of Flexure Mechanism Design. EPFL Press, 2017.

[13] W. Dong, L. Sun, and Z. Du. Stiffness research on a high-precision, largeworkspace parallel mechanism with compliant joints. Precision Engineering, 32(3):222-231, 2008.

[14] C. Ericson. Real-Time Collision Detection. CRC Press, 2004.

[15] D. Farhadi Machekposhti, N. Tolou, and J. L. Herder. A Review on Compliant Joints and Rigid-Body Constant Velocity Universal Joints Toward the Design of Compliant Homokinetic Couplings. Fournal of Mechanical Design, 137(3):032301, 2015.

[16] N. Fazenda, E. Lubrano, S. Rossopoulos, and R. Clavel. Calibration of the 6 DOF high-precision flexure parallel robot "Sigma 6". Technical report, Fraunhofer, 2006.

[17] J. T. Feddema, C. G. Keller, and R. T. Howe. Experiments in micromanipulation and cad-driven microassembly. Technical report, Sandia National Labs., Albuquerque, NM (United States), 1997.

[18] Z. Ferková, M. Franko, J. Kuchta, and P. Rafajdus. Electromagnetic design of ironless permanent magnet synchronous linear motor. In International Symposium on Power Electronics, Electrical Drives, Automation and Motion, pages 721-726. IEEE, 2008.

[19] K. G. P. Folkersma, S. E. Boer, D. M. Brouwer, J. L. Herder, and H. M. J. R. Soemers. A 2-DOF large stroke flexure based positioning mechanism. In International Design Engineering Technical Conferences and Computers and Information in Engineering Conference, volume 45035, pages 221-228. American Society of Mechanical Engineers, 2012.

[20] P. C. Fourie and A. A. Groenwold. The particle swarm optimization algorithm in size and shape optimization. Structural and Multidisciplinary Optimization, 23(4):259-267, 2002.

[21] M. I. Frecker, G. K. Ananthasuresh, S. Nishiwaki, N. Kikuchi, and S. Kota. Topological Synthesis of Compliant Mechanisms Using Multi-Criteria Optimization. Journal of Mechanical Design, 119(2):238-245, 1997.

[22] F. Gao and L. Han. Implementing the Nelder-Mead simplex algorithm with adaptive parameters. Computational Optimization and Applications, 51(1):259-277, 2012.

[23] W. Gellert, S. Gottwald, M. Hellwich, H. Kästner, and H. Küstner. Analytic geometry of space. In The VNR Concise Encyclopedia of Mathematics, 
chapter 24, pages 530-547. Springer, 1975.

[24] S. Gottschalk, M. C. Lin, and D. Manocha. OBBTree: A Hierarchical Structure for Rapid Interference Detection. In Proceedings of the 23rd Annual Conference on Computer Graphics and Interactive Techniques, pages 171-180. ACM, 1996.

[25] Y. X. Gu, G. Z. Zhao, H. W. Zhang, Z. Kang, and R. V. Grandhi. Buckling design optimization of complex built-up structures with shape and size variables. Structural and Multidisciplinary Optimization, 19(3):183-191, 2000.

[26] P. Guigue and O. Devillers. Fast and Robust Triangle-Triangle Overlap Test Using Orientation Predicates. Journal of graphics tools, 8(1):25-32, 2003.

[27] K. Gunnink, R. G. K. M. Aarts, and D. M. Brouwer. Performance optimization of large stroke flexure hinges for high stiffness and eigenfrequency. In Proceedings of the 28th Annual Meeting of the American Society for Precision Engineering (ASPE), Saint Paul, MN, 2013.

[28] R. T. Haftka and Z. Gürdal. Elements of structural optimization, volume 11. Springer Science \& Business Media, 2012.

[29] L. C. Hale. Principles and techniques for designing precision machines. $\mathrm{PhD}$ thesis, 1999.

[30] G. Hao, X. Kong, and R. L. Reuben. A nonlinear analysis of spatial compliant parallel modules: Multi-beam modules. Mechanism and Machine Theory, 46(5):680-706, 2011.

[31] J. A. Haringx. The cross-spring pivot as a constructional element. Flow, Turbulence and Combustion, 1(1):313-332, 1949.

[32] S. Henein. Flexure delicacies. Mechanical Sciences, 3:1-4, 2012.

[33] S. Henein, P. Spanoudakis, S. Droz, L. I. Myklebust, and E. Onillon. Flexure pivot for aerospace mechanisms. In 10th European Space Mechanisms and Tribology Symposium, pages 285-288, San Sebastian, Spain, 2003. Citeseer.

[34] J. Hesselbach, J. Wrege, A. Raatz, and O. Becker. Aspects on design of high precision parallel robots. Assembly Automation, 24(1):49-57, 2004.

[35] Z. Hongzhe and B. Shusheng. Accuracy characteristics of the generalized cross-spring pivot. Mechanism and Machine Theory, 45(10):1434-1448, 2010.

[36] J. B. Hopkins. Design of parallel flexure systems via freedom and constraint topologies (FACT). PhD thesis, Massachusetts Institute of Technology, 2007.

[37] J. B. Hopkins and M. L. Culpepper. Synthesis of multi-degree of freedom, parallel flexure system concepts via Freedom and Constraint Topology (FACT) - Part I: Principles. Precision Engineering, 34(2):259-270, 2010. 
[38] J. B. Hopkins and R. M. Panas. A family of flexures that eliminate underconstraint in nested large-stroke flexure systems. In 13th International Conference on European Society for Precision Engineering and Nanotechnology, Berlin, 2013.

[39] L. L. Howell. Compliant Mechanisms. John Wiley \& Sons, 2001.

[40] L. L. Howell, S. P. Magleby, and B. M. Olsen. Handbook of compliant mechanisms. John Wiley \& Sons, 2013.

[41] P. M. Hubbard. Approximating Polyhedra with Spheres for Time-Critical Collision Detection. ACM Transaction on Graphics (TOG), 15(3):179-210, 1996.

[42] A. Industries. ALIO HH brochure 2019. https://www.optoprim.it/wpcontent/uploads/2020/02/ALIO-HH-Brochure-2019.pdf, 2019.

[43] R. V. Jones. Some uses of elasticity in instrument design. Fournal of Scientific Instruments, 39(5):193-203, 1962.

[44] R. V. Jones. Instruments and experiences: papers on measurement and instrument design. Chichester: Wiley, 1988.

[45] J. B. Jonker and J. P. Meijaard. SPACAR - Computer Program for Dynamic Analysis of Flexible Spatial Mechanisms and Manipulators. In Multibody Systems Handbook, pages 123-143. Springer, Berlin, Heidelberg, 1990.

[46] A. Kaveh and S. Talatahari. Size optimization of space trusses using Big Bang-Big Crunch algorithm. Computers \& Structures, 87(17-18):1129-1140, 2009.

[47] M. P. Koster, P. C. J. N. Rosielle, and E. A. G. Reker. Constructies voor het nauwkeurig bewegen en positioneren. Deel 7. Beheersen van vrijheidsgraden. Deel 1. Mikroniek, 32(4):100-105, 1992.

[48] B. Krijnen and D. M. Brouwer. Flexures for large stroke electrostatic actuation in MEMS. Journal of Micromechanics and Microengineering, 24(1):015006, 2013.

[49] G. Krishnan, C. Kim, and S. Kota. An Intrinsic Geometric Framework for the Building Block Synthesis of Single Point Compliant Mechanisms. Journal of Mechanisms and Robotics, 3(1):011001, 2011.

[50] T. Kubela, A. Pochyly, and V. Singule. Investigation of position accuracy of industrial robots and online methods for accuracy improvement in machining processes. In 2015 International Conference on Electrical Drives and Power Electronics (EDPE), pages 385-388. IEEE, 2015.

[51] I. Kuric, V. Tlach, Z. Ságová, M. Císar, and I. Gritsuk. Measurement of industrial robot pose repeatability. MATEC Web of Conferences, 244:01015, 2018. 
[52] H. Kwakernaak. H2-Optimization - Theory and Applications to Robust Control Design. IFAC Proceedings Volumes, 33(14):437-448, 2000.

[53] E. R. Laithwaite and S. A. Nasar. Linear-Motion Electrical Machines. Proceedings of the IEEE, 58:531-540, 1970.

[54] J. Li, H. Huang, and T. Morita. Stepping piezoelectric actuators with large working stroke for nano-positioning systems: A review. Sensors and Actuators, A: Physical, 292:39-51, 2019.

[55] C.-H. Lin, S.-K. Hung, M.-Y. Chen, S.-T. Li, and L.-C. Fu. A novel high precision electromagnetic flexure-suspended positioning stage with an eddy current damper. In 2008 International Conference on Control, Automation and Systems, pages 771-776. IEEE, 2008.

[56] N. Lobontiu and J. S. N. Paine. Design of Circular Cross-Section CornerFilleted Flexure Hinges for Three-Dimensional Compliant Mechanisms. Journal of Mechanical Design, 124(3):479-484, 2002.

[57] K. Marković and S. Zelenika. Optimized cross-spring pivot configurations with minimized parasitic shifts and stiffness variations investigated via nonlinear FEA. Mechanics Based Design of Structures and Machines, 45(3):380-394, 2017.

[58] J. P. Meijaard. Fluid-Conveying Flexible Pipes Modeled by LargeDeflection Finite Elements in Multibody Systems. Fournal of Computational and Nonlinear Dynamics, 9(1):011008, 2013.

[59] J. P. Meijaard, D. M. Brouwer, and J. B. Jonker. Analytical and experimental investigation of a parallel leaf spring guidance. Multibody System Dynamics, 23(1):77-97, 2010.

[60] J.-P. Merlet. Parallel Robots, volume 128. Spring Science \& Business Media, 2005.

[61] T. Möller. A Fast Triangle-Triangle Intersection Test. Fournal of graphics tools, 2(2):25-30, 1997.

[62] Y.-M. Moon and S. Kota. Design of compliant parallel kinematic machines. In International Design Engineering Technical Conferences and Computers and Information in Engineering Conference, volume 5, pages 35-41, Montreal, 2002.

[63] M. Moore and J. Wilhelms. Collision Detection and Response for Computer Animation. Computer Graphic, 22(4):289-298, 1988.

[64] S. Murugesan. An Overview of Electric Motors for Space Applications. IEEE Transactions on Industrial Electronics and Control Instrumentation, IECI-28(4):260-265, 1981.

[65] M. Naves, R. G. K. M. Aarts, and D. M. Brouwer. Large stroke high 
support stiffness flexure-based universal joint. In 32nd Annual meeting of the American Society for Precision Engineering (ASPE), pages 398-412, Charlotte, NC, 2016.

[66] M. Naves, R. G. K. M. Aarts, and D. M. Brouwer. Large stroke three degreeof-freedom spherical flexure joint. In 17th International Conference of the European Society for Precision Engineering and Nanotechnology (EUSPEN), pages 97-98, Hannover, 2017.

[67] M. Naves, R. G. K. M. Aarts, and D. M. Brouwer. Efficient collision detection method for flexure mechanisms comprising deflected leafsprings. Fournal of Mechanisms and Robotics, 10(6), 2018.

[68] M. Naves, R. G. K. M. Aarts, and D. M. Brouwer. Large stroke high offaxis stiffness three degree of freedom spherical flexure joint. Precision Engineering, 56:422-431, 2019.

[69] M. Naves, D. M. Brouwer, and R. G. K. M. Aarts. Building Block-Based Spatial Topology Synthesis Method for Large-Stroke Flexure Hinges. Journal of Mechanisms and Robotics, 9(4):041006, 2017.

[70] M. Naves, M. Nijenhuis, W. B. J. Hakvoort, and D. M. Brouwer. Flexurebased 60 degrees stroke actuator suspension for a high torque iron core motor. Precision Engineering, 63:105-114, 2020.

[71] J. A. Nelder and R. Mead. A simplex method for function minimization. The Computer fournal, 7(4):308-313, 1965.

[72] M. Nijenhuis and D. M. Brouwer. A closed-form model for the support stiffness of spatial flexure strips with limited twist. In International Design Engineering Technical Conferences and Computers and Information in Engineering Conference, Charlotte, NC, 2017.

[73] M. Nijenhuis, J. P. Meijaard, and D. M. Brouwer. A Spatial Parametric Model for the Nonlinear stiffness characteristics of flexure strips. In ASME 2018 International Design Engineering Technical Conferences and Computers and Information in Engineering Conference, Quebec City, 2018.

[74] M. Nijenhuis, J. P. Meijaard, and D. M. Brouwer. A spatial closed-form nonlinear stiffness model for sheet flexures based on a mixed variational principle including third-order effects. Precision Engineering, 66:429-444, 2020.

[75] M. Nijenhuis, J. P. Meijaard, D. Mariappan, J. L. Herder, D. M. Brouwer, and S. Awtar. An analytical formulation for the lateral support stiffness of a spatial flexure strip. Fournal of mechanical design, 139(5):051401, 2017.

[76] C. B. W. Pedersen, T. Buhl, and O. Sigmund. Topology synthesis of largedisplacement compliant mechanisms. International fournal for numerical 
methods in engineering, 50(12):2683-2705, 2001.

[77] X. Pei, J. Yu, G. Zong, S. Bi, and H. Su. The modeling of cartwheel flexural hinges. Mechanism and Machine Theory, 44(10):1900-1909, 2009.

[78] A. Pérez-Calpena, E. Sánchez-Blanco, I. Martínez-Delgado, M. L. GarcíaVargas, X. Arrillaga, H. Aube, A. Gil de Paz, M. Maldonado, J. Gallego, J. Iglesias-Páramo, E. Carrasco, F. M. Sánchez-Moreno, J. Vilchez, and R. Cedazo. MEGARA: high-precision alignment system for gluing fibers and microlenses. Advances in Optical and Mechanical Technologies for Telescopes and Instrumentation II, 9912:99125L, 2016.

[79] Physik Instrumente (PI). 6-Axis Precision Alignment System. https: //www.pi-usa.us/fileadmin/user_upload/pi_us/files/product_datasheets/ F206_Fiber_Alignment_System.pdf 2019.

[80] Physik Instrumente (PI). Hexapod Positioning Systems. https://static.physikinstrumente.com/fileadmin/user_upload/physik_inst rumente/files/CAT/PI-CAT136-Hexapod-Positioning-Systems.pdf, 2019.

[81] Physik Instrumente (PI). Q-Motion ${ }^{\circledR}$ SpaceFAB Q-845. https:// www.physikinstrumente.nl/fileadmin/user_upload/physik_instrumente/ files/datasheets/Q-845-Datasheet.pdf, 2019.

[82] N. K. Pyschny. Auslegung und Optimierung von festkörpergelenkbasierten Parallelkinematiken für die Montage von optischen Komponenten. $\mathrm{PhD}$ thesis, Rheinisch-Westfälischen Technischen Hochschule Aachen, 2014.

[83] R. Ravaud, G. Lemarquand, and V. Lemarquand. Ironless permanent magnet motors: Three-dimensional analytical calculation. In IEEE International Electric Machines and Drives Conference, pages 947-952, 2009.

[84] P. C. J. N. Rosielle and E. A. G. Reker. Constructieprincipes 1, bedoeld voor het nauwkeurig bewegen en positioneren. Lecture notes, 4007, 2000.

[85] P. Schellekens, N. Rosielle, H. Vermeulen, M. Vermeulen, S. Wetzels, and W. Pril. Design for Precision: Current Status and Trends. CIRP Annals, 47(2):557-586, 1998.

[86] J. Schwarze. Interscection of two lines in three-space. In Graphics Gems I, pages 296-341. Academic Press, Inc., 1990.

[87] B. Shusheng, Z. Hongzhe, and Y. Jingjun. Modeling of a Cartwheel Flexural Pivot. Fournal of Mechanical Design, 131(6):061010, 2009.

[88] SmarAct. SMARPOD 110.45. https://www.smaract.com/smarpod/ product/smarpod-110-45 2019.

[89] D. R. Smith, K. Souccar, D. M. Gale, M. Lucero, E. Hernández, A. Olmos, M. E. Moreno, M. Cuq, P. Noire, R. Pawlowski, and T. Roux. Factory characterization testing of a large precision hexapod for the LMT/GTM. 
In Advances in Optical and Mechanical Technologies for Telescopes and Instrumentation III, volume 10706, page 1070649. International Society for Optics and Photonics, 2018.

[90] S. T. Smith. Flexures: Elements of Elastic Mechanisms. Crc Press, London, 2000.

[91] H. A. Sodano, J.-S. Bae, D. J. Inman, and W. K. Belvin. Improved Concept and Model of Eddy Current Damper. Fournal of Vibration and Acoustics, 128(3):294-302, 2006.

[92] H. Soemers. Design Principles for Precision Mechanisms. T-Pointprint, Enschede, 2010.

[93] K. Spanner and B. Koc. Piezoelectric motors, an overview. Actuators, 5(1), 2016.

[94] Tecnotion. Frameless Torque Motor Series. https://www.tecnotion.com/ downloads/catalogue-torque.pdf 2018.

[95] Tecnotion. Torque motor manual. https://www.tecnotion.com/ downloads/torque-motors-manual.pdf 2018.

[96] M. Teschner, S. Kimmerle, B. Heidelberger, G. Zachmann, L. Raghupathi, A. Fuhrmann, M. P. Cani, F. Faure, N. Magnenat-Thalmann, W. Strasser, and E. al. Collision detection for deformable objects. In Computer Graphics Forum, volume 24, pages 61-81. Blackwell Publishing Ltd., 2005.

[97] J. H. Timmer Arends, K. H. J. Voss, W. B. J. Hakvoort, and R. G. K. M. Aarts. Kinematic Calibration of a Six DOF Flexure-based Parallel Manipulator. In ECCOMAS Thematic Conference on Multibody Dynamics, pages 199-211, Prague, 2017.

[98] B. P. Trease, Y.-M. Moon, and S. Kota. Design of Large-Displacement Compliant Joints. Fournal of Mechanical Design, 127(4):788-798, 2005.

[99] Uddeholm. Uddeholm Stavax ESR. https://www.uddeholm.com/files/PB Uddeholm_stavax_esr_english.pdf 2019.

[100] Universal Robots. E-Series brochure. https://www.universal-robots.com/ media/1802432/e-series-brochure.pdf 2021.

[101] G. van den Bergen. Proximity queries and penetration depth computation on 3D game objects. In Game developers conference, volume 170, 2001.

[102] N. P. Van Dijk, K. Maute, M. Langelaar, and F. Van Keulen. Level-set methods for structural topology optimization: A review. Structural and Multidisciplinary Optimization, 48(3):437-472, 2013.

[103] J. Van Eijk. On the design of plate-spring mechanisms. PhD thesis, Delft University of Technology, 1985. 
[104] I. Vardi, L. Rubbert, R. Bitterli, N. Ferrier, M. Kahrobaiyan, B. Nussbaumer, and S. Henein. Theory and design of spherical oscillator mechanisms. Precision Engineering, 51:499-513, 2018.

[105] L. Wang, P. K. Basu, and J. P. Leiva. Automobile body reinforcement by finite element optimization. Finite Elements in Analysis and Design, 40(8):879-893, 2004.

[106] N. Wavre and X. Thouvenin. Voice-coil actuators in space. In 6th European Space Mechanisms and Tribology Symposium, volume 374, page 227, 1995.

[107] R. Weller. New geometric data structures for collision detection and haptics. Springer Science \& Business Media, 2013.

[108] D. H. Wiersma, S. E. Boer, R. G. K. M. Aarts, and D. M. Brouwer. Design and Performance Optimization of Large Stroke Spatial Flexures. Journal of Computational and Nonlinear Dynamics, 9(1):011016, 2014.

[109] W. H. Wittrick. The properties of crossed flexure pivots, and the influence of the point at which the strips cross. The Aeronautical Quarterly, 2(4):272292, 1951.

[110] T.-L. Wu, J.-H. Chen, and S.-H. Chang. A six-dof prismatic-sphericalspherical parallel compliant nanopositioner. IEEE Transactions on Ultrasonics, Ferroelectrics, and Frequency Control, 55(12):2544-2551, 2008.

[111] X. Ye, L. Huang, L. Wang, and H. Xing. An Improved Algorithm for Triangle to Triangle Intersection Test. In IEEE International Conference on Information and Automation, pages 2689-2694, 2015.

[112] S. Zelenika and F. De Bona. Analytical and experimental characterisation of high-precision flexural pivots subjected to lateral loads. Precision Engineering, 26(4):381-388, 2002. 



\section{Research contributions}

\section{Patents}

- M. Naves, R.G.K.M. Aarts and D.M. Brouwer, Guidance element with internal conduit between two constructional parts, IPC no. E05D11/00 (Patent No. NL2017880B1), 2018.

- M. Naves, R.G.K.M. Aarts and D.M. Brouwer, Guidance element with internal conduit between two constructional parts, IPC no. E05D11/00 (Patent No. WO2018097729), 2018.

\section{Journal publications (scientific)}

- M. Naves, D.M. Brouwer and R.G.K.M. Aarts. Building block-based spatial topology synthesis method for large-stroke flexure hinges, fournal of Mechanisms and Robotics, 9(4):041006, 2017.

- M. Naves, R.G.K.M. Aarts and D.M. Brouwer. Efficient collision detection method for flexure mechanisms comprising deflected leafsprings, fournal of Mechanisms and Robotics, 10(6), 2018.

- M. Naves, R.G.K.M. Aarts and D.M. Brouwer. Large stroke high off-axis stiffness three degree of freedom spherical flexure joint, Precision Engineering, 56:422-431, 2019.

- M. Naves, M. Nijenhuis, W.B.J. Hakvoort and D.M. Brouwer. Flexurebased large stroke actuator suspension for high torque iron core motor, Precision Engineering, 63:105-114, 2020.

- F. Tajdari, A.P. Berkhoff, M. Naves, M. Nijenhuis and A. de Boer. A lowprofile flexural displacement-converter mechanism for piezoelectric stack actuators, Sensors \& Actuators A: Physical, 313:112198, 2020. 
- M. Naves, M. Nijenhuis, B. Seinhorst, W.B.J. Hakvoort and D.M. Brouwer. T-Flex: A fully flexure-based large range of motion precision hexapod, Precision Engineering, submitted.

- M. Nijenhuis, J.P. Meijaard, M. Naves, R.G.K.M. Aarts and D.M. Brouwer. Third-order stiffness formulation for sheet flexures using an energy-based discretization approach (EDA): numerical beam element, Computational Mechanics, submitted.

- J. Rommers, M. Naves, D.M. Brouwer and J.L. Herder. A flexure-based linear guide with torsion reinforcement structures, fournal of Mechanisms and Robotics, submitted.

\section{Journal publications (professional)}

- M. Naves, R.G.K.M. Aarts and D.M. Brouwer. Large-stroke flexure hinges: Building-block-based spatial topology synthesis method, Mikroniek, 57(3):59, 2017.

- M. Naves, R.G.K.M. Aarts and D.M. Brouwer. Maintaining high support stiffness: Large-stroke spherical flexure joint, Mikroniek, 59(3):17-21, 2019.

- M. Naves, M. Nijenhuis, W.B.J. Hakvoort and D.M. Brouwer. Large-stroke, flexure-based: Suspension for an iron-core, high-torque motor, Mikroniek, 59(6):22-26, 2019.

\section{Conference publications}

- M. Naves, D.M. Brouwer and R.G.K.M. Aarts. Multibody-based topology synthesis method for large-stroke flexure hinges, In: Proceedings of the International Design Engineering Technical Conference and Computers and Information in Engineering Conference, IDETC2016 (ASME), Charlotte, NC, USA, 2016.

- M. Naves, D.M. Brouwer and R.G.K.M. Aarts. Multibody-based topology synthesis method for large-stroke flexure hinges, In: Proceedings of the 3 th Dutch Society for Precision Engineering conference (DSPE), NL, 2016.

- M. Naves, R.G.K.M. Aarts and D.M. Brouwer. Large stroke high support stiffness flexure based universal joint, In: Proceedings of the 32nd annual 
meeting of the American Society for Precision Engineering (ASPE), Charlotte, NC, USA, 2017.

- M. Naves, R.G.K.M. Aarts and D.M. Brouwer. Large-stroke three degreeof-freedom spherical flexure joint, In: Proceedings of the 17th international conference of the European Society for Precision Engineering and Nanotechnology (EUSPEN), Hannover, DE, 2017.

- B. Boers, M. Naves, L.A.G. Rodriquez and D.M. Brouwer. Inverted curved flexure hinge with torsional reinforcements in a printed prosthetic finger, In: Proceedings of the 33rd annual meeting of the American Society for Precision Engineering (ASPE), Las Vegas, NV, USA, 2018.

- J. Rommers, M. Naves, D.M. Brouwer and J.L. Herder. A large range spatial linear guide with torsion reinforcement structures, In: Proceedings of the International Design Engineering Technical Conference and Computers and Information in Engineering Conference, IDETC2018 (ASME), Quebec City, CA, 2018.

- L.A.G. Rodriquez, M. Naves and D.M. Brouwer. 3D-printed flexure-based finger joints for anthropomorphic hands, In: IEEE/RSf International Conference on Intelligent Robots and Systems (IROS), Madrid, ES, 2018.

- M. Naves, M. Nijenhuis, W.B.J. Hakvoort and D.M. Brouwer. Flexurebased large stroke actuator suspension for an high torque iron core motor, In: Proceedings of the 19th international conference of the European Society for Precision Engineering and Nanotechnology (EUSPEN), BilBao, ES, 2019.

- M. Naves, W.B.J. Hakvoort, M. Nijenhuis and D.M. Brouwer. T-Flex: Large range of motion fully flexure based 6-DOF hexapod, In: Proceedings of the 20th international conference of the European Society for Precision Engineering and Nanotechnology (EUSPEN), Geneva, CH, 2020.

- M. Nijenhuis, M. Naves, W.B.J. Hakvoort and D.M. Brouwer. Efficient modeling for the design of a large-stroke fully flexure-based 6-DOF hexapod, In: Proceedings of the 35th annual meeting of the American Society for Precision Engineering (ASPE), Minneapolis, MN, USA, 2020.

\section{Awards}

- Best Fast Forward Presentation Award at the MR-6 Compliant Mechanisms and Micro/Nano Mechanisms, International Design Engineering Technical 
Conference and Computers and Information in Engineering Conference, IDETC2016 (ASME), Charlotte, NC, USA, 2016.

- Best Oral Presentation Award, 19th EUSPEN International Conference \& Exhibition, 2019, Bilbao, ES, 2019.

- Precision Engineering Editor's Choice Article, Awarded to M. Naves, R.G.K.M. Aarts and D.M. Brouwer for the Paper entitled "Large stroke high off-axis stiffness three degree of freedom spherical flexure joint”, Volume 56, 2019, pp. $422-431$. 


\section{Dankwoord}

Via dit dankwoord wil ik iedereen bedanken die heeft bijgedragen aan dit proefschrift en de leuke tijd tijdens mijn promotieonderzoek.

In de eerste plaats wil ik Dannis, Ronald, Wouter en Marijn bedanken voor de begeleiding en ondersteuning tijdens mijn promotieonderzoek en het schrijven van onze publicaties. Verder wil ik jullie, samen met Leo en Axel, danken voor de (technische) ondersteuning bij het ontwikkelen van diverse experimentele opstellingen en demonstrators.

De leden van de promotiecommissie, Hans Vermeulen, Just Herder, Ton van den Boogaard en Richard Loendersloot, bedankt voor het lezen van mijn proefschrift, het leveren van feedback en het beoordelen van dit werk.

Jalisa, bedankt voor de steun in de afgelopen jaren en het ontwerp van de cover.

Martin, Marijn en Jan, het was me de afgelopen jaren een genoegen om met jullie samen te werken en het kantoor te delen. Dit heeft een grote bijdrage geleverd aan zowel het proefschrift als de werksfeer. Bedankt!

Tot slot wil ik alle andere leden van de MS3 afdeling, de collega's van de Precision Engineering en de voormalige Werktuigbouwkundige Automatisering vakgroep, en iedereen die direct, indirect, bewust of onbewust een bijdrage hebben geleverd bedanken. 

Flexure-based mechanisms are widely used in many small stroke precision applications due to the absence of play and friction, resulting in highly deterministic and predictable behavior. However, for large stroke applications, flexure-based mechanisms are often avoided due to the strong decrease in support stiffness and load-bearing capacity when subjected to large deflections.

To improve the potential of flexurebased mechanisms for large stroke applications, an optimization strategy is combined with design principles for flexure-based systems to obtain new design topologies for flexure-based equivalents of traditional bearings. This approach has been used to develop and optimize a large stroke flexure-based revolute, universal and spherical joint. To demonstrate the potential of large stroke flexure joints, a fully flexure-based 6-DOF hexapod robot is presented, utilizing large range of motion flexure joints. 